Internet Engineering Task Force (IETF)

Request for Comments: 6234

Obsoletes: 4634

Updates: 3174

Category: Informational

ISSN : 2070-1721

D. Eastlake 3rd Huawei

T. Hansen

AT\&T Labs

May 2011

US Secure Hash Algorithms

(SHA and SHA-based HMAC and HKDF)

Abstract

The United States of America has adopted a suite of Secure Hash Algorithms (SHAs), including four beyond SHA-1, as part of a Federal Information Processing Standard (FIPS), namely SHA-224, SHA-256, SHA-384, and SHA-512. This document makes open source code performing these SHA hash functions conveniently available to the Internet community. The sample code supports input strings of arbitrary bit length. Much of the text herein was adapted by the authors from FIPS 180-2.

This document replaces RFC 4634, fixing errata and adding code for an HMAC-based extract-and-expand Key Derivation Function, HKDF (RFC 5869). As with RFC 4634, code to perform SHA-based Hashed Message Authentication Codes (HMACs) is also included.

Status of This Memo

This document is not an Internet Standards Track specification; it is published for informational purposes.

This document is a product of the Internet Engineering Task Force (IETF). It represents the consensus of the IETF community. It has received public review and has been approved for publication by the Internet Engineering Steering Group (IESG). Not all documents approved by the IESG are a candidate for any level of Internet Standard; see Section 2 of RFC 5741.

Information about the current status of this document, any errata, and how to provide feedback on it may be obtained at http://www.rfc-editor.org/info/rfc6234. 
Copyright Notice

Copyright (c) 2011 IETF Trust and the persons identified as the document authors. All rights reserved.

This document is subject to BCP 78 and the IETF Trust's Legal Provisions Relating to IETF Documents (http://trustee.ietf.org/license-info) in effect on the date of publication of this document. Please review these documents carefully, as they describe your rights and restrictions with respect to this document. Code Components extracted from this document must include Simplified BSD License text as described in section $4 . e$ of the Trust Legal Provisions and are provided without warranty as described in the Simplified BSD License. 
Table of Contents

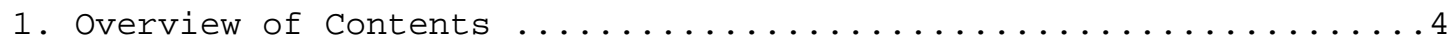

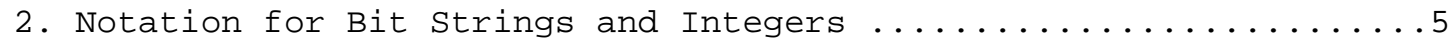

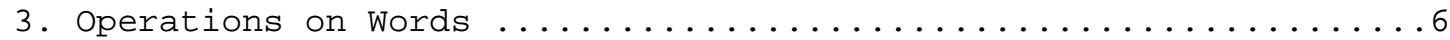

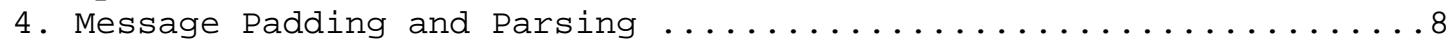

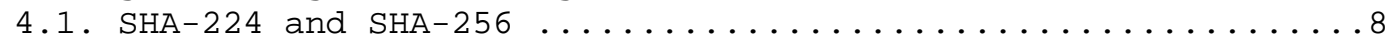

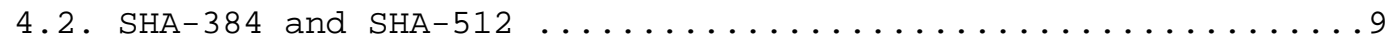

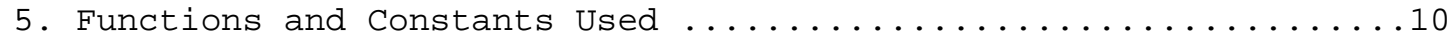

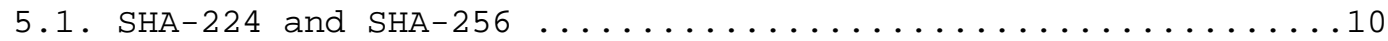

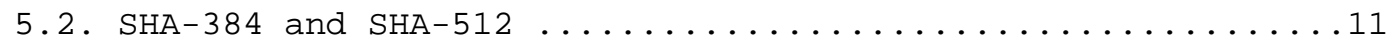

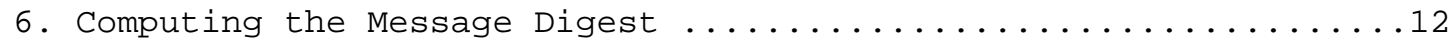

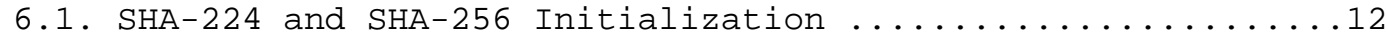

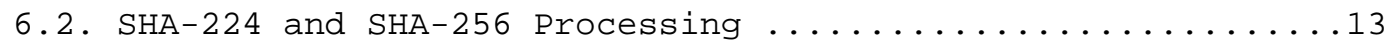

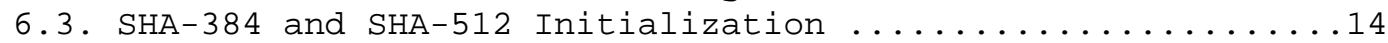

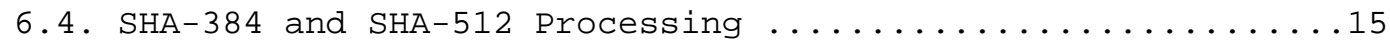

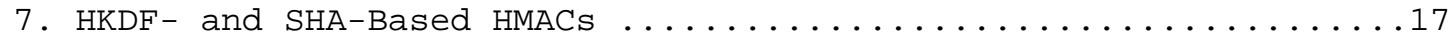

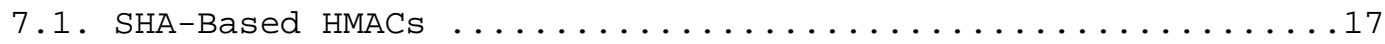

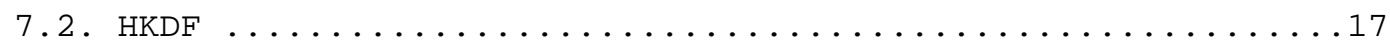

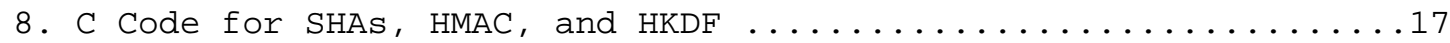

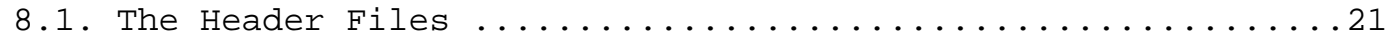

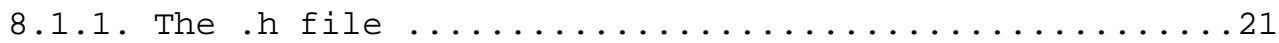

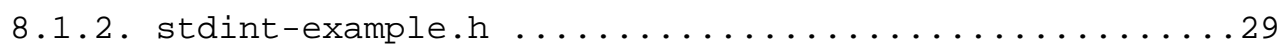

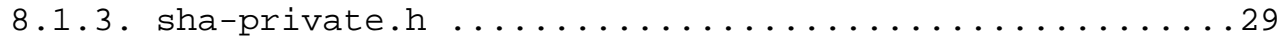

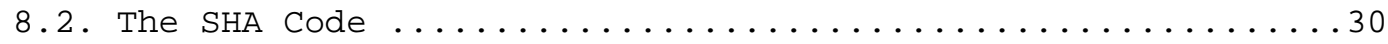

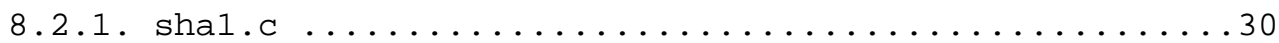

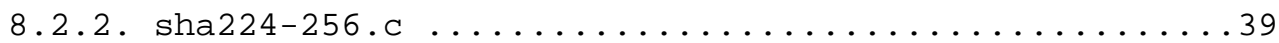

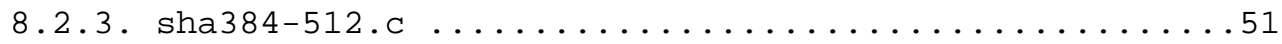

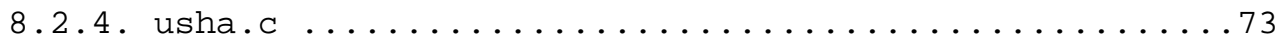

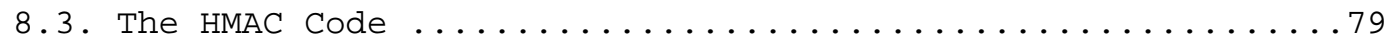

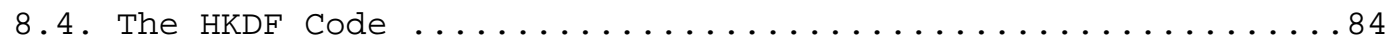

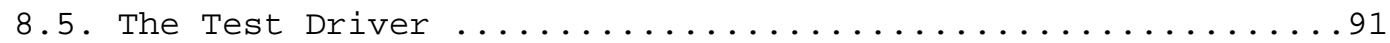

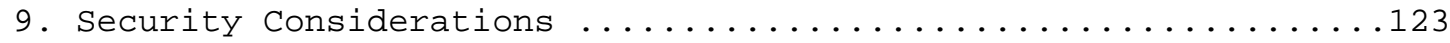

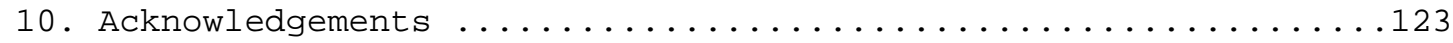

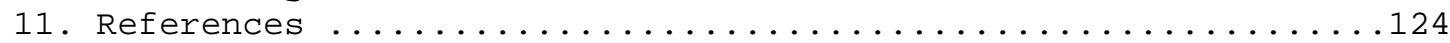

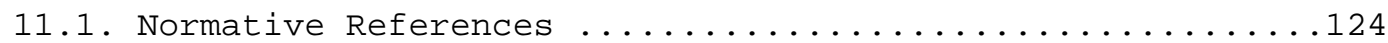

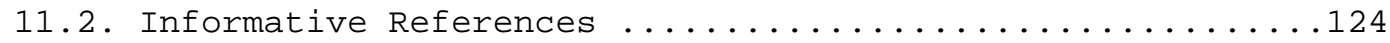

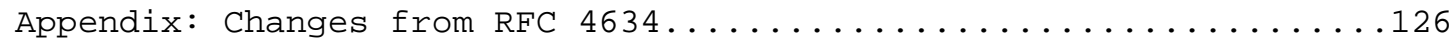




\section{Overview of Contents}

This document includes specifications for the United States of America (USA) Federal Information Processing Standard (FIPS) Secure Hash Algorithms (SHAs), code to implement the SHAs, code to implement HMAC (Hashed Message Authentication Code, [RFC2104]) based on the SHAs, and code to implement HKDF (HMAC-based Key Derivation Function, [RFC5869]) based on HMAC. Specifications for HMAC and HKDF are not included as they appear elsewhere in the RFC series [RFC2104] [RFC5869].

NOTE: Much of the text below is taken from [SHS], and the assertions of the security of the hash algorithms described therein are made by the US Government, the author of [SHS], not by the listed authors of this document. See also [RFC6194] concerning the security of SHA-1.

The text below specifies Secure Hash Algorithms, SHA-224 [RFC3874], SHA-256, SHA-384, and SHA-512, for computing a condensed representation of a message or a data file. (SHA-1 is specified in [RFC3174].) When a message of any length $<2 \wedge 64$ bits (for SHA-224 and SHA-256) or $<2^{\wedge} 128$ bits (for SHA-384 and SHA-512) is input to one of these algorithms, the result is an output called a message digest. The message digests range in length from 224 to 512 bits, depending on the algorithm. Secure Hash Algorithms are typically used with other cryptographic algorithms, such as digital signature algorithms and keyed-hash authentication codes, the generation of random numbers [RFC4086], or in key derivation functions.

The algorithms specified in this document are called secure because it is computationally infeasible to (1) find a message that corresponds to a given message digest, or (2) find two different messages that produce the same message digest. Any change to a message in transit will, with very high probability, result in a different message digest. This will result in a verification failure when the Secure Hash Algorithm is used with a digital signature algorithm or a keyed-hash message authentication algorithm.

The code provided herein supports input strings of arbitrary bit length. SHA-1's sample code from [RFC3174] has also been updated to handle input strings of arbitrary bit length. Permission is granted for all uses, commercial and non-commercial, of this code.

This document obsoletes [RFC4634], and the changes from that RFC are summarized in the Appendix. 
ASN.1 OIDs (Object Identifiers) for the SHA algorithms, taken from [RFC4055], are as follows:

id-shal OBJECT IDENTIFIER $::=\{$ iso(1)

identified-organization(3) oiw(14)

secsig(3) algorithms(2) 26$\}$

id-sha224 OBJECT IDENTIFIER $::=\quad\{\{$ joint-iso-itu-t (2)

country(16) us(840) organization(1) gov(101)

csor(3) nistalgorithm(4) hashalgs(2) 4 \}

id-sha256 OBJECT IDENTIFIER $::=$ \{ joint-iso-itu-t(2)

country(16) us(840) organization(1) gov(101)

id-sha384 OBJECT IDENTIFIER ::= \{ joint-iso-itu-t (2) country(16) us(840) organization(1) gov(101) csor(3) nistalgorithm(4) hashalgs(2) 2 \}

id-sha512 OBJECT IDENTIFIER $::=$ \{ joint-iso-itu-t(2) country(16) us(840) organization(1) gov(101) csor(3) nistalgorithm(4) hashalgs(2) 3 \}

Section 2 below defines the terminology and functions used as building blocks to form these algorithms. Section 3 describes the fundamental operations on words from which these algorithms are built. Section 4 describes how messages are padded up to an integral multiple of the required block size and then parsed into blocks. Section 5 defines the constants and the composite functions used to specify the hash algorithms. Section 6 gives the actual specification for the SHA-224, SHA-256, SHA-384, and SHA-512 functions. Section 7 provides pointers to the specification of HMAC keyed message authentication codes and to the specification of an extract-and-expand key derivation function based on HMAC.

Section 8 gives sample code for the SHA algorithms, for SHA-based HMACs, and for HMAC-based extract-and-expand key derivation function.

2. Notation for Bit Strings and Integers

The following terminology related to bit strings and integers will be used:

a. A hex digit is an element of the set $\{0,1, \ldots, 9, A, \ldots, F\}$. A hex digit is the representation of a 4-bit string. Examples: 7 $=0111, A=1010$.

b. A word equals a 32-bit or 64-bit string that may be represented as a sequence of 8 or 16 hex digits, respectively. To convert a word to hex digits, each 4-bit string is converted to its hex equivalent as described in (a) above. Example: 
$10100001000000111111111000100011=$ A103FE23.

Throughout this document, the "big-endian" convention is used when expressing both 32-bit and 64-bit words, so that within each word the most significant bit is shown in the leftmost bit position.

c. An integer may be represented as a word or pair of words.

An integer between 0 and $2^{\wedge} 32$ - 1 inclusive may be represented as a 32-bit word. The least significant four bits of the integer are represented by the rightmost hex digit of the word representation. Example: the integer $291=2^{\wedge} 8+2^{\wedge} 5+2^{\wedge} 1+2^{\wedge} 0=256+32+2+1$ is represented by the hex word 00000123 .

The same holds true for an integer between 0 and $2^{\wedge} 64-1$ inclusive, which may be represented as a 64-bit word.

If $\mathrm{z}$ is an integer, $0<=\mathrm{z}<2^{\wedge} 64$, then $\mathrm{z}=\left(2^{\wedge} 32\right) \mathrm{x}+\mathrm{y}$ where $0<=x<2^{\wedge} 32$ and $0<=y<2^{\wedge} 32$. Since $x$ and $y$ can be represented as words $X$ and $Y$, respectively, $\mathrm{Z}$ can be represented as the pair of words $(X, Y)$.

Again, the "big-endian" convention is used and the most significant word is in the leftmost word position for values represented by multiple-words.

d. block = 512-bit or 1024-bit string. A block (e.g., B) may be represented as a sequence of 32-bit or 64-bit words.

\section{Operations on Words}

The following logical operators will be applied to words in all four hash operations specified herein. SHA-224 and SHA-256 operate on 32-bit words while SHA-384 and SHA-512 operate on 64-bit words.

In the operations below, $\mathrm{x}<<\mathrm{n}$ is obtained as follows: discard the leftmost $\mathrm{n}$ bits of $\mathrm{x}$ and then pad the result with $\mathrm{n}$ zeroed bits on the right (the result will still be the same number of bits). Similarly, $x>>n$ is obtained as follows: discard the rightmost $n$ bits of $x$ and then prepend the result with $n$ zeroed bits on the left (the result will still be the same number of bits).

a. Bitwise logical word operations

$$
\begin{aligned}
& \mathrm{X} \text { AND } \mathrm{Y}=\text { bitwise logical "and" of } \mathrm{X} \text { and } \mathrm{Y} . \\
& \mathrm{X} \text { OR } \mathrm{Y}=\text { bitwise logical "inclusive-or" of } \mathrm{X} \text { and } \mathrm{Y} .
\end{aligned}
$$




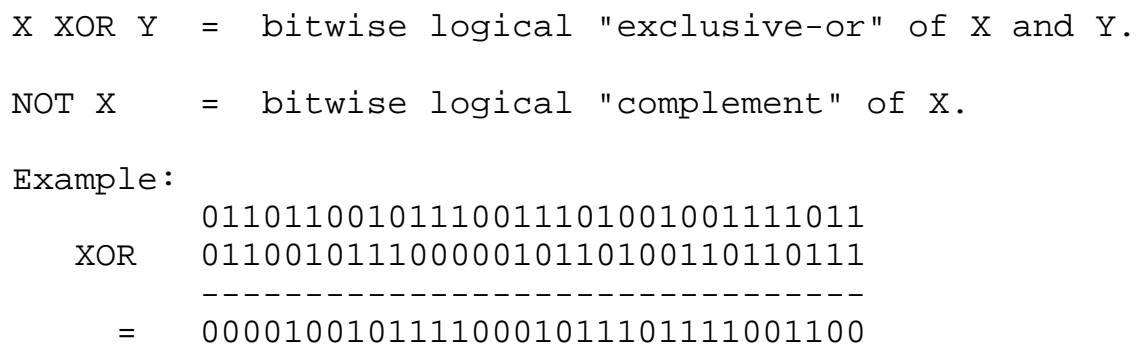

b. The operation $X+Y$ is defined as follows: words $X$ and $Y$ represent $\mathrm{w}$-bit integers $\mathrm{x}$ and $\mathrm{y}$, where $0<=\mathrm{x}<2^{\wedge} \mathrm{w}$ and $0<=\mathrm{y}<2^{\wedge} \mathrm{w}$. For positive integers $\mathrm{n}$ and $\mathrm{m}$, let

$n \bmod m$

be the remainder upon dividing $n$ by $m$. Compute

$$
z=(x+y) \bmod 2^{\wedge} w
$$

Then $0<=\mathrm{Z}<2^{\wedge} \mathrm{W}$. Convert $\mathrm{z}$ to a word, $\mathrm{Z}$, and $\operatorname{define~} \mathrm{Z}=\mathrm{X}+\mathrm{Y}$.

c. The right shift operation $\operatorname{SHR}^{\wedge} \mathrm{n}(\mathrm{x})$, where $\mathrm{x}$ is a $\mathrm{w}^{-\mathrm{b}}$ it word and $\mathrm{n}$ is an integer with $0<=\mathrm{n}<\mathrm{w}$, is defined by

$$
\operatorname{SHR}^{\wedge} \mathrm{n}(\mathrm{x})=\mathrm{x}>>\mathrm{n}
$$

d. The rotate right (circular right shift) operation $\operatorname{ROTR}^{\wedge} \mathrm{n}(\mathrm{x})$, where $\mathrm{x}$ is $\mathrm{a} \mathrm{w}$-bit word and $\mathrm{n}$ is an integer with $0<=\mathrm{n}<\mathrm{w}$, is defined by

$$
\operatorname{ROTR}^{\wedge} \mathrm{n}(\mathrm{x})=(\mathrm{x}>>\mathrm{n}) \text { OR }(\mathrm{x}<<(\mathrm{w}-\mathrm{n}))
$$

e. The rotate left (circular left shift) operation ROTL^n(x), where $\mathrm{x}$ is a $\mathrm{w}$-bit word and $\mathrm{n}$ is an integer with $0<=\mathrm{n}<\mathrm{w}$, is defined by

$$
\operatorname{ROTL}^{\wedge} \mathrm{n}(\mathrm{X})=(\mathrm{x}<<\mathrm{n}) \operatorname{OR}(\mathrm{x}>>(\mathrm{w}-\mathrm{n}))
$$

Note the following equivalence relationships, where $\mathrm{w}$ is fixed in each relationship:

$$
\begin{aligned}
& \operatorname{ROTL}^{\wedge} n(x)=\operatorname{ROTR}^{\wedge}(w-n)(x) \\
& \operatorname{ROTR}^{\wedge} n(x)=\operatorname{ROTL}^{\wedge}(w-n)(x)
\end{aligned}
$$




\section{Message Padding and Parsing}

The hash functions specified herein are used to compute a message digest for a message or data file that is provided as input. The message or data file should be considered to be a bit string. The length of the message is the number of bits in the message (the empty message has length 0). If the number of bits in a message is a multiple of 8, for compactness we can represent the message in hex. The purpose of message padding is to make the total length of a padded message a multiple of 512 for SHA-224 and SHA-256 or a multiple of 1024 for SHA-384 and SHA-512.

The following specifies how this padding shall be performed. As a summary, a "1" followed by m "0"s followed by a 64-bit or 128-bit integer are appended to the end of the message to produce a padded message of length $512{ }^{*} \mathrm{n}$ or $1024{ }^{*} \mathrm{n}$. The appended integer is the length of the original message. The padded message is then processed by the hash function as n 512-bit or 1024-bit blocks.

4.1. SHA-224 and SHA-256

Suppose a message has length $\mathrm{L}<2^{\wedge} 64$. Before it is input to the hash function, the message is padded on the right as follows:

a. "1" is appended. Example: if the original message is "01010000", this is padded to "010100001".

b. $K$ "O"s are appended where $K$ is the smallest, non-negative solution to the equation

$(L+1+K) \bmod 512=448$

c. Then append the 64-bit block that is L in binary representation. After appending this block, the length of the message will be a multiple of 512 bits.

Example: Suppose the original message is the bit string

$01100001011000100110001101100100 \quad 01100101$

After step (a) this gives

01100001011000100110001101100100011001011 


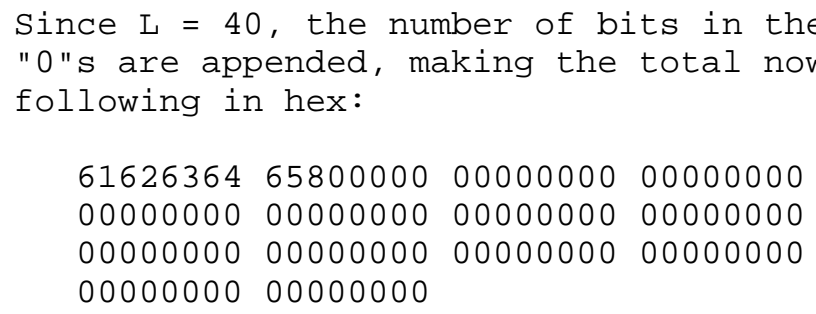

The 64-bit representation of $\mathrm{L}=40$ is hex 0000000000000028 . Hence the final padded message is the following hex

$\begin{array}{llll}61626364 & 65800000 & 00000000 & 00000000 \\ 00000000 & 00000000 & 00000000 & 00000000 \\ 00000000 & 00000000 & 00000000 & 00000000 \\ 00000000 & 00000000 & 00000000 & 00000028\end{array}$

4.2. SHA-384 and SHA-512

Suppose a message has length $L<2 \wedge 128$. Before it is input to the hash function, the message is padded on the right as follows:

a. "1" is appended. Example: if the original message is "01010000", this is padded to "010100001".

b. K "O"s are appended where $\mathrm{K}$ is the smallest, non-negative solution to the equation

$(L+1+K) \bmod 1024=896$

c. Then append the 128-bit block that is L in binary representation. After appending this block, the length of the message will be a multiple of 1024 bits.

Example: Suppose the original message is the bit string

0110000101100010011000110110010001100101

After step (a) this gives

01100001011000100110001101100100011001011 


Since $\mathrm{L}=40$, the number of bits in the
"0"s are appended, making the total now
following in hex:
$\quad 61626364658000000000000000000000$
00000000000000000000000000000000
00000000000000000000000000000000
00000000000000000000000000000000
00000000000000000000000000000000
00000000000000000000000000000000
00000000000000000000000000000000

The 128-bit representation of $\mathrm{L}=40$ is hex 0000000000000000 0000000000000028 . Hence the final padded message is the following hex:

$\begin{array}{llll}61626364 & 65800000 & 00000000 & 00000000 \\ 00000000 & 00000000 & 00000000 & 00000000 \\ 00000000 & 00000000 & 00000000 & 00000000 \\ 00000000 & 00000000 & 00000000 & 00000000 \\ 00000000 & 00000000 & 00000000 & 00000000 \\ 00000000 & 00000000 & 00000000 & 00000000 \\ 00000000 & 00000000 & 00000000 & 00000000 \\ 00000000 & 00000000 & 00000000 & 00000028\end{array}$

5. Functions and Constants Used

The following subsections give the six logical functions and the table of constants used in each of the hash functions.

5.1. SHA-224 and SHA-256

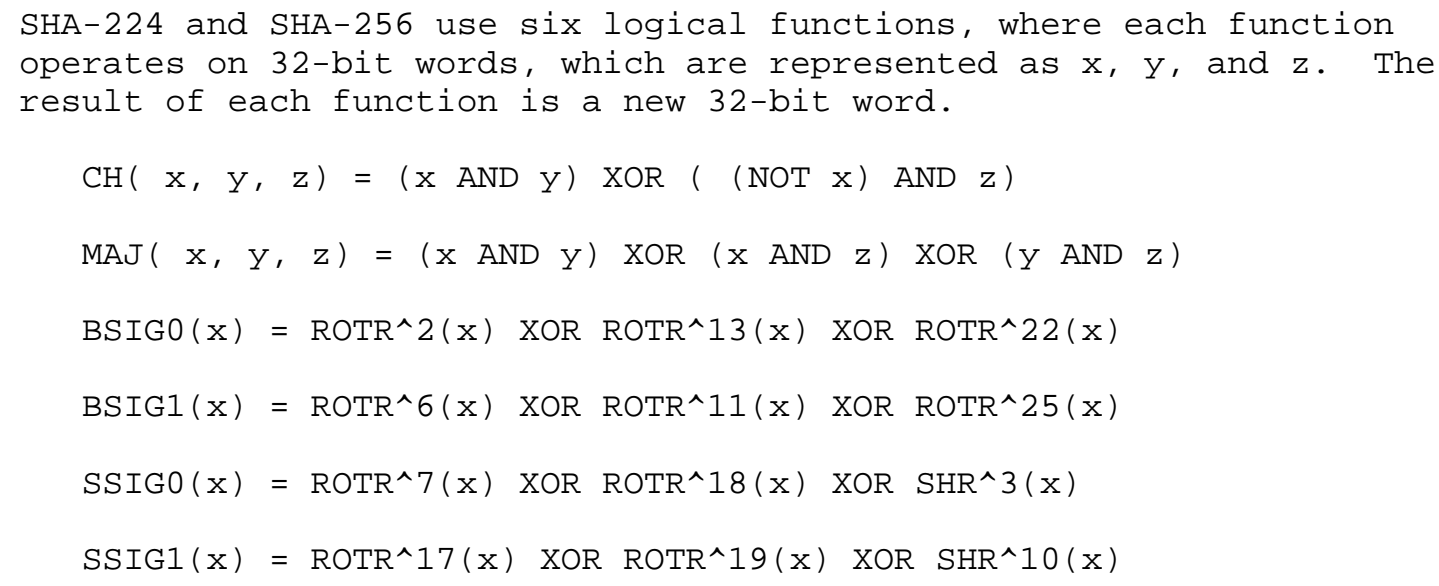


SHA-224 and SHA-256 use the same sequence of sixty-four constant 32-bit words, K0, K1, ..., K63. These words represent the first 32 bits of the fractional parts of the cube roots of the first sixtyfour prime numbers. In hex, these constant words are as follows (from left to right):

$\begin{array}{llll}428 a 2 f 98 & 71374491 & b 5 c 0 f b c f & \text { e } 955 d b a 5 \\ 3956 c 25 b & 59 f 111 f 1 & 923 f 82 a 4 & \text { ablc5ed5 } \\ \text { d807aa98 } & 12835 b 01 & 243185 b e & 550 c 7 d c 3 \\ 72 b e 5 d 74 & 80 d e b 1 f e & 9 b d c 06 a 7 & c 19 b f 174 \\ \text { e49b69c1 } & \text { efbe4786 } & 0 f c 19 d c 6 & 240 c a 1 c c \\ 2 d e 92 c 6 f & 4 a 7484 a a & 5 c b 0 a 9 d c & 76 f 988 d a \\ 983 e 5152 & \text { a831c66d } & \text { b00327c8 } & \text { bf597fc7 } \\ \text { c6e00bf3 } & \text { d5a79147 } & 06 c a 6351 & 14292967 \\ 27 b 70 a 85 & 2 e 1 b 2138 & 4 d 2 c 6 d f c & 53380 d 13 \\ 650 a 7354 & 766 a 0 a b b & 81 c 2 c 92 e & 92722 c 85 \\ \text { a2bfe8a1 } & \text { a81a664b } & \text { c24b8b70 } & \text { c76c51a3 } \\ \text { d192e819 } & \text { d6990624 } & \text { f40e3585 } & 106 a a 070 \\ 19 a 4 c 116 & 1 e 376 c 08 & 2748774 c & 34 b 0 b c b 5 \\ 391 c 0 c b 3 & 4 e d 8 a a 4 a & 5 b 9 c c a 4 f & 682 e 6 f f 3 \\ 748 f 82 e e & 78 a 5636 f & 84 c 87814 & 8 c c 70208 \\ 90 b e f f f a & \text { a4506ceb } & \text { bef9a3f7 } & \text { c67178f2 }\end{array}$

5.2. SHA-384 and SHA-512

SHA-384 and SHA-512 each use six logical functions, where each function operates on 64-bit words, which are represented as $x, y$, and $z$. The result of each function is a new 64-bit word.

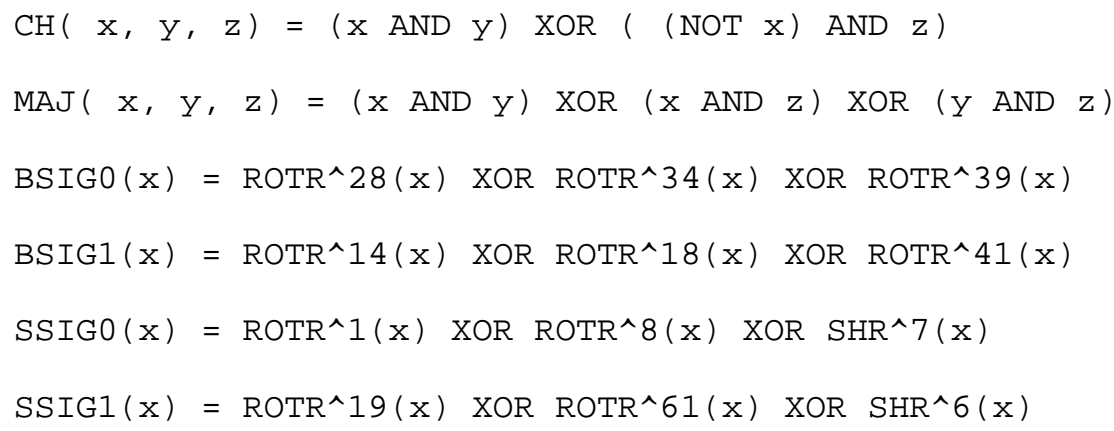


428a2f98d728ae22 7137449123ef65cd b5c0fbcfec4d3b2f e9b5dba58189dbbc 3956 c25bf348b538 59f111flb605d019 923f82a 4 afl94f9b ablc5ed5da6d8118 d807aa98a3030242 12835b0145706fbe 243185be4ee4b28c 550c7dc3d5ffb4e2 72be5d74f27b896f 80deb1fe3b1696b1 9bdc06a725c71235 c19bf174cf692694 e49b69c19ef14ad2 efbe4786384f25e3 0fc19dc68b8cd5b5 $240 c a 1 c c 77 a c 9 c 65$ 2 de92c6f592b0275 4a7484aa6ea6e483 5cb0a9dcbd41fbd4 76f988da831153b5 983 e5152ee66dfab a831c66d2db43210 b00327c898fb213f bf597fc7beef0ee 4 c6e00bf33da88fc2 d5a79147930aa725 06ca6351e003826f 142929670a0e6e70 27b70a8546d22ffc 2e1b21385c26c926 4d2c6dfc5ac42aed 53380d139d95b3df $650 \mathrm{a} 73548 \mathrm{baf} 63 \mathrm{de} 766 \mathrm{a} 0 \mathrm{abb3c77b2a8}$ 81c2c92e47edaee6 92722c851482353b a2bfe8a14cf10364 a81a664bbc423001 c24b8b70d0f89791 c76c51a30654be30 d192e819d6ef5218 d69906245565a910 f40e35855771202a 106aa07032bbd1b8 $19 \mathrm{a} 4 \mathrm{c} 116 \mathrm{~b} 8 \mathrm{~d} 2 \mathrm{~d} 0 \mathrm{c} 8$ 1e376c085141ab53 $2748774 \mathrm{cdf} 8 \mathrm{eeb} 99 \quad 34 \mathrm{~b} 0 \mathrm{bcb} 5 \mathrm{e} 19 \mathrm{~b} 48 \mathrm{a} 8$ $391 \mathrm{c} 0 \mathrm{cb} 3 \mathrm{c} 5 \mathrm{c} 95 \mathrm{a} 63$ 4ed8aa4ae3418acb 5b9cca4f7763e373 682e6ff3d6b2b8a3 $748 f 82$ ee 5 defb2fc 78a5636f43172f60 84c87814a1f0ab72 8cc702081a6439ec 90 befffa23631e28 a4506cebde82bde9 bef9a3f7b2c67915 c67178f2e372532b ca273eceea26619c d186b8c721c0c207 eada7dd6cde0eble f57d4f7fee6ed178 $06 \mathrm{f} 067 \mathrm{aa} 72176 \mathrm{fba} 0 \mathrm{a} 637 \mathrm{dc5a2c898a6}$ 113f9804bef90dae 1b710b35131c471b $28 \mathrm{db} 77 \mathrm{f} 523047 \mathrm{~d} 84$ 32caab7b40c72493 3c9ebe0a15c9bebc 431d67c49c100d4c 4 cc5d4becb3e42b6 597f299cfc657e2a 5fcb6fab3ad6faec 6c44198c4a475817

6. Computing the Message Digest

The output of each of the secure hash functions, after being applied to a message of $\mathrm{N}$ blocks, is the hash quantity $\mathrm{H}(\mathrm{N})$. For SHA-224 and SHA-256, H(i) can be considered to be eight 32-bit words, H(i) 0 , $\mathrm{H}(i) 1, \ldots \mathrm{H}(\mathrm{i}) 7$. For SHA-384 and SHA-512, it can be considered to be eight 64-bit words, H(i) $0, \mathrm{H}(\mathrm{i}) 1, \ldots, \mathrm{H}(\mathrm{i}) 7$.

As described below, the hash words are initialized, modified as each message block is processed, and finally concatenated after processing the last block to yield the output. For SHA-256 and SHA-512, all of the $\mathrm{H}(\mathrm{N})$ variables are concatenated while the SHA-224 and SHA-384 hashes are produced by omitting some from the final concatenation.

6.1. SHA-224 and SHA-256 Initialization

For SHA-224, the initial hash value, $H(0)$, consists of the following 32-bit words in hex:
$\mathrm{H}(0) 0=\mathrm{c} 1059 \mathrm{ed} 8$
$\mathrm{H}(0) 1=367 \mathrm{~cd} 507$
$\mathrm{H}(0) 2=3070 \mathrm{dd} 17$
$\mathrm{H}(0) 3=\mathrm{f} 70 \mathrm{e} 5939$
$\mathrm{H}(0) 4=\mathrm{ffc} 00 \mathrm{~b} 31$
$\mathrm{H}(0) 5=68581511$
$\mathrm{H}(0) 6=64 \mathrm{fg} 8 \mathrm{fa} 7$
$\mathrm{H}(0) 7=$ befa 4 fa 4 
For SHA-256, the initial hash value, H(0), consists of the following eight 32-bit words, in hex. These words were obtained by taking the first 32 bits of the fractional parts of the square roots of the first eight prime numbers.
$\mathrm{H}(0) 0=6 \mathrm{a} 09 \mathrm{e} 667$
$\mathrm{H}(0) 1=\mathrm{bb} 67 \mathrm{ae} 85$
$\mathrm{H}(0) 2=3 \mathrm{c} 6 \mathrm{ef} 372$
$\mathrm{H}(0) 3=\mathrm{a} 54 \mathrm{ff5} 3 \mathrm{a}$
$\mathrm{H}(0) 4=510 \mathrm{e} 527 \mathrm{f}$
$\mathrm{H}(0) 5=9 \mathrm{~b} 05688 \mathrm{C}$
$\mathrm{H}(0) 6=1 \mathrm{f} 83 \mathrm{~d} 9 \mathrm{ab}$
$\mathrm{H}(0) 7=5$ be $0 \mathrm{~cd} 19$

6.2. SHA-224 and SHA-256 Processing

SHA-224 and SHA-256 perform identical processing on message blocks and differ only in how $\mathrm{H}(0)$ is initialized and how they produce their final output. They may be used to hash a message, M, having a length of L bits, where $0<=\mathrm{L}<2^{\wedge} 64$. The algorithm uses (1) a message schedule of sixty-four 32-bit words, (2) eight working variables of 32 bits each, and (3) a hash value of eight 32-bit words.

The words of the message schedule are labeled W0, W1, ..., W63. The eight working variables are labeled a, b, c, d, e, f, $g$, and h. The words of the hash value are labeled H(i)0, H(i) 1, ... H(i) 7, which will hold the initial hash value, $\mathrm{H}(0)$, replaced by each successive intermediate hash value (after each message block is processed), $\mathrm{H}(\mathrm{i})$, and ending with the final hash value, $\mathrm{H}(\mathrm{N})$, after all $\mathrm{N}$ blocks are processed. They also use two temporary words, T1 and T2.

The input message is padded as described in Section 4.1 above, then parsed into 512-bit blocks that are considered to be composed of sixteen 32-bit words M(i)0, M(i)1,..., M(i)15. The following computations are then performed for each of the $\mathrm{N}$ message blocks. All addition is performed modulo $2^{\wedge} 32$.

For $i=1$ to $\mathrm{N}$

1. Prepare the message schedule $\mathrm{W}$ :

For $t=0$ to 15

Wt $=M(i) t$

For $t=16$ to 63

$W t=\operatorname{SSIGI}(W(t-2))+W(t-7)+\operatorname{SSIGO}(W(t-15))+W(t-16)$ 
2. Initialize the working variables:

$$
\begin{aligned}
& \mathrm{a}=\mathrm{H}(\mathrm{i}-1) 0 \\
& \mathrm{~b}=\mathrm{H}(\mathrm{i}-1) 1 \\
& \mathrm{c}=\mathrm{H}(\mathrm{i}-1) 2 \\
& \mathrm{~d}=\mathrm{H}(\mathrm{i}-1) 3 \\
& \mathrm{e}=\mathrm{H}(\mathrm{i}-1) 4 \\
& \mathrm{f}=\mathrm{H}(\mathrm{i}-1) 5 \\
& \mathrm{~g}=\mathrm{H}(\mathrm{i}-1) 6 \\
& \mathrm{~h}=\mathrm{H}(\mathrm{i}-1) 7
\end{aligned}
$$

3. Perform the main hash computation:

For $t=0$ to 63

$$
\begin{aligned}
& \mathrm{T} 1=\mathrm{h}+\mathrm{BSIG}(\mathrm{e})+\mathrm{CH}(\mathrm{e}, \mathrm{f}, \mathrm{g})+\mathrm{Kt}+\mathrm{Wt} \\
& \mathrm{T} 2=\operatorname{BSIG0}(\mathrm{a})+\operatorname{MAJ}(\mathrm{a}, \mathrm{b}, \mathrm{c}) \\
& \mathrm{h}=\mathrm{g} \\
& \mathrm{g}=\mathrm{f} \\
& \mathrm{f}=\mathrm{e} \\
& \mathrm{e}=\mathrm{d}+\mathrm{T} 1 \\
& \mathrm{~d}=\mathrm{C} \\
& \mathrm{C}=\mathrm{b} \\
& \mathrm{b}=\mathrm{a} \\
& \mathrm{a}=\mathrm{T} 1+\mathrm{T} 2
\end{aligned}
$$

4. Compute the intermediate hash value $\mathrm{H}(\mathrm{i})$

$\mathrm{H}(\mathrm{i}) \mathrm{O}=\mathrm{a}+\mathrm{H}(\mathrm{i}-1) 0$

$\mathrm{H}(\mathrm{i}) 1=\mathrm{b}+\mathrm{H}(\mathrm{i}-1) 1$

$\mathrm{H}(\mathrm{i}) 2=\mathrm{C}+\mathrm{H}(\mathrm{i}-1) 2$

$\mathrm{H}(\mathrm{i}) 3=\mathrm{d}+\mathrm{H}(\mathrm{i}-1) 3$

$\mathrm{H}(\mathrm{i}) 4=\mathrm{e}+\mathrm{H}(\mathrm{i}-1) 4$

$\mathrm{H}(\mathrm{i}) 5=\mathrm{f}+\mathrm{H}(\mathrm{i}-1) 5$

$H(i) 6=g+H(i-1) 6$

$\mathrm{H}(\mathrm{i}) 7=\mathrm{h}+\mathrm{H}(\mathrm{i}-1) 7$

After the above computations have been sequentially performed for all of the blocks in the message, the final output is calculated. For SHA-256, this is the concatenation of all of $H(N) 0, H(N) 1$, through $\mathrm{H}(\mathrm{N})$ 7. For SHA-224, this is the concatenation of $H(N) 0, H(N) 1$, through $\mathrm{H}(\mathrm{N}) 6$.

6.3. SHA-384 and SHA-512 Initialization

For SHA-384, the initial hash value, H(0), consists of the following eight 64-bit words, in hex. These words were obtained by taking the first 64 bits of the fractional parts of the square roots of the ninth through sixteenth prime numbers. 
$\mathrm{H}(0) 0=$ cbbb9d5dc1059ed
$\mathrm{H}(0) 1=629 \mathrm{a} 292 \mathrm{a} 367 \mathrm{~cd} 507$
$\mathrm{H}(0) 2=9159015 \mathrm{a} 3070 \mathrm{dd} 17$
$\mathrm{H}(0) 3=152 \mathrm{fecd} 8 \mathrm{f} 70 \mathrm{e} 5939$
$\mathrm{H}(0) 4=67332667 \mathrm{ffc} 00 \mathrm{~b} 31$
$\mathrm{H}(0) 5=8 \mathrm{eb} 44 \mathrm{a} 8768581511$
$\mathrm{H}(0) 6=\mathrm{db0c} 2 \mathrm{e} 0 \mathrm{~d} 64 \mathrm{f} 98 \mathrm{fa} 7$
$\mathrm{H}(0) 7=47 \mathrm{~b} 5481 \mathrm{dbefa} 4 \mathrm{fa} 4$

For SHA-512, the initial hash value, H(0), consists of the following eight 64-bit words, in hex. These words were obtained by taking the first 64 bits of the fractional parts of the square roots of the first eight prime numbers.
$\mathrm{H}(0) 0=6 \mathrm{a} 09 \mathrm{e} 667 \mathrm{f} 3 \mathrm{bcc} 908$
$\mathrm{H}(0) 1=\mathrm{bb} 67 \mathrm{ae} 8584 \mathrm{caa} 73 \mathrm{~b}$
$\mathrm{H}(0) 2=3 \mathrm{c} 6 \mathrm{ef} 372 \mathrm{fe} 94 \mathrm{f} 82 \mathrm{~b}$
$\mathrm{H}(0) 3=\mathrm{a} 54 \mathrm{ff5} 3 \mathrm{a} 5 \mathrm{fld} 36 \mathrm{f} 1$
$\mathrm{H}(0) 4=510 \mathrm{e} 527 \mathrm{fade} 682 \mathrm{~d} 1$
$\mathrm{H}(0) 5=9 \mathrm{~b} 05688 \mathrm{c} 2 \mathrm{~b} 3 \mathrm{e} 6 \mathrm{c} 1 \mathrm{f}$
$\mathrm{H}(0) 6=1 \mathrm{f} 83 \mathrm{~d} 9 \mathrm{abfb} 41 \mathrm{bd} 6 \mathrm{~b}$
$\mathrm{H}(0) 7=5 \mathrm{be} 0 \mathrm{~cd} 19137 \mathrm{e} 2179$

6.4. SHA-384 and SHA-512 Processing

SHA-384 and SHA-512 perform identical processing on message blocks and differ only in how $\mathrm{H}(0)$ is initialized and how they produce their final output. They may be used to hash a message, $M$, having a length of L bits, where $0<=\mathrm{L}<2^{\wedge} 128$. The algorithm uses (1) a message schedule of eighty 64-bit words, (2) eight working variables of 64 bits each, and (3) a hash value of eight 64-bit words.

The words of the message schedule are labeled W0, W1, ..., W79. The eight working variables are labeled a, b, c, d, e, f, $g$, and h. The words of the hash value are labeled H(i) $0, \mathrm{H}(\mathrm{i}) 1, \ldots, \mathrm{H}(\mathrm{i}) 7$, which will hold the initial hash value, $\mathrm{H}(0)$, replaced by each successive intermediate hash value (after each message block is processed), $\mathrm{H}(\mathrm{i})$, and ending with the final hash value, $\mathrm{H}(\mathrm{N})$ after all $\mathrm{N}$ blocks are processed.

The input message is padded as described in Section 4.2 above, then parsed into 1024-bit blocks that are considered to be composed of sixteen 64-bit words M(i)0, M(i)1,..., M(i) 15. The following computations are then performed for each of the $\mathrm{N}$ message blocks. All addition is performed modulo $2^{\wedge} 64$. 


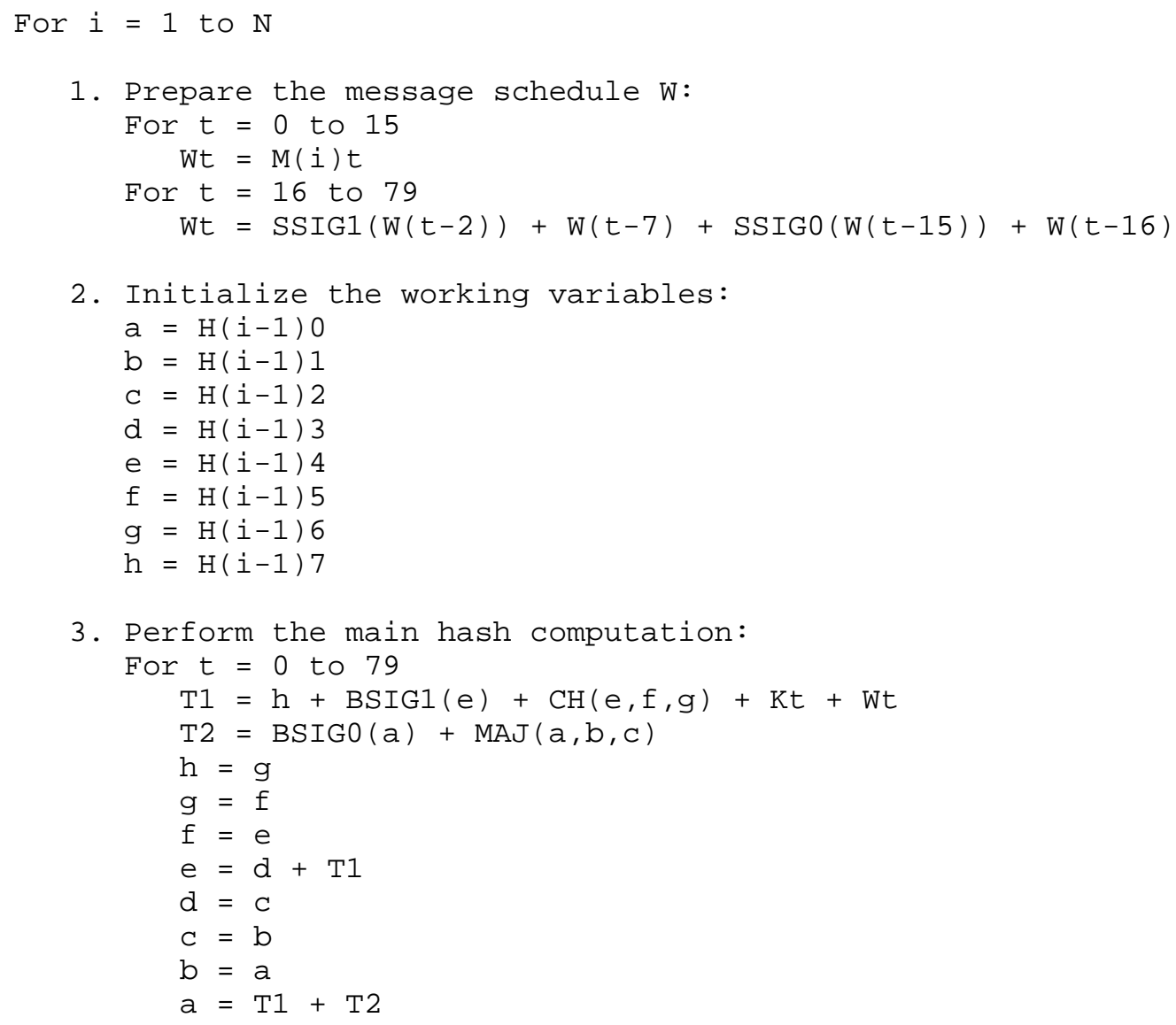

4. Compute the intermediate hash value $H(i)$

$\mathrm{H}(\mathrm{i}) 0=\mathrm{a}+\mathrm{H}(\mathrm{i}-1) 0$

$\mathrm{H}(\mathrm{i}) 1=\mathrm{b}+\mathrm{H}(\mathrm{i}-1) 1$

$\mathrm{H}(\mathrm{i}) 2=\mathrm{C}+\mathrm{H}(\mathrm{i}-1) 2$

$\mathrm{H}(\mathrm{i}) 3=\mathrm{d}+\mathrm{H}(\mathrm{i}-1) 3$

$\mathrm{H}(\mathrm{i}) 4=\mathrm{e}+\mathrm{H}(\mathrm{i}-1) 4$

$\mathrm{H}(\mathrm{i}) 5=\mathrm{f}+\mathrm{H}(\mathrm{i}-1) 5$

$\mathrm{H}(\mathrm{i}) 6=\mathrm{g}+\mathrm{H}(\mathrm{i}-1) 6$

$\mathrm{H}(\mathrm{i}) 7=\mathrm{h}+\mathrm{H}(\mathrm{i}-1) 7$

After the above computations have been sequentially performed for all of the blocks in the message, the final output is calculated. For SHA-512, this is the concatenation of all of $\mathrm{H}(\mathrm{N}) 0, \mathrm{H}(\mathrm{N}) 1$, through $\mathrm{H}(\mathrm{N})$ 7. For SHA-384, this is the concatenation of $H(N) 0, H(N) 1$, through $\mathrm{H}(\mathrm{N}) 5$. 
7. HKDF - and SHA-Based HMACS

Below are brief descriptions and pointers to more complete descriptions and code for (1) SHA-based HMACs and (2) an HMAC-based extract-and-expand key derivation function. Both HKDF and HMAC were devised by Hugo Krawczyk.

\subsection{SHA-Based HMACs}

HMAC is a method for computing a keyed MAC (Message Authentication Code) using a hash function as described in [RFC2104]. It uses a key to mix in with the input text to produce the final hash.

Sample code is also provided, in Section 8.3 below, to perform HMAC based on any of the SHA algorithms described herein. The sample code found in [RFC2104] was written in terms of a specified text size. Since SHA is defined in terms of an arbitrary number of bits, the sample HMAC code has been written to allow the text input to HMAC to have an arbitrary number of octets and bits. A fixed-length interface is also provided.

\section{2. $\mathrm{HKDF}$}

HKDF is a specific Key Derivation Function (KDF), that is, a function of initial keying material from which the KDF derives one or more cryptographically strong secret keys. HKDF, which is described in [RFC5869], is based on HMAC.

Sample code for HKDF is provided in section 8.4 below.

8. C Code for SHAs, HMAC, and HKDF

Below is a demonstration implementation of these secure hash functions in C. Section 8.1 contains the header file sha.h that declares all constants, structures, and functions used by the SHA and HMAC functions. It includes conditionals based on the state of definition of USE_32BIT_ONLY that, if that symbol is defined at compile time, avoids 64-bit operations. It also contains shaprivate.h that provides some declarations common to all the SHA functions. Section 8.2 contains the C code for sha1.c, sha224-256.C, sha384-512.C, and usha.c. Section 8.3 contains the C code for the HMAC functions, and Section 8.4 contains the C code for HKDF. Section 8.5 contains a test driver to exercise the code.

For each of the digest lengths $\$ \$ \$$, there is the following set of constants, a structure, and functions: 


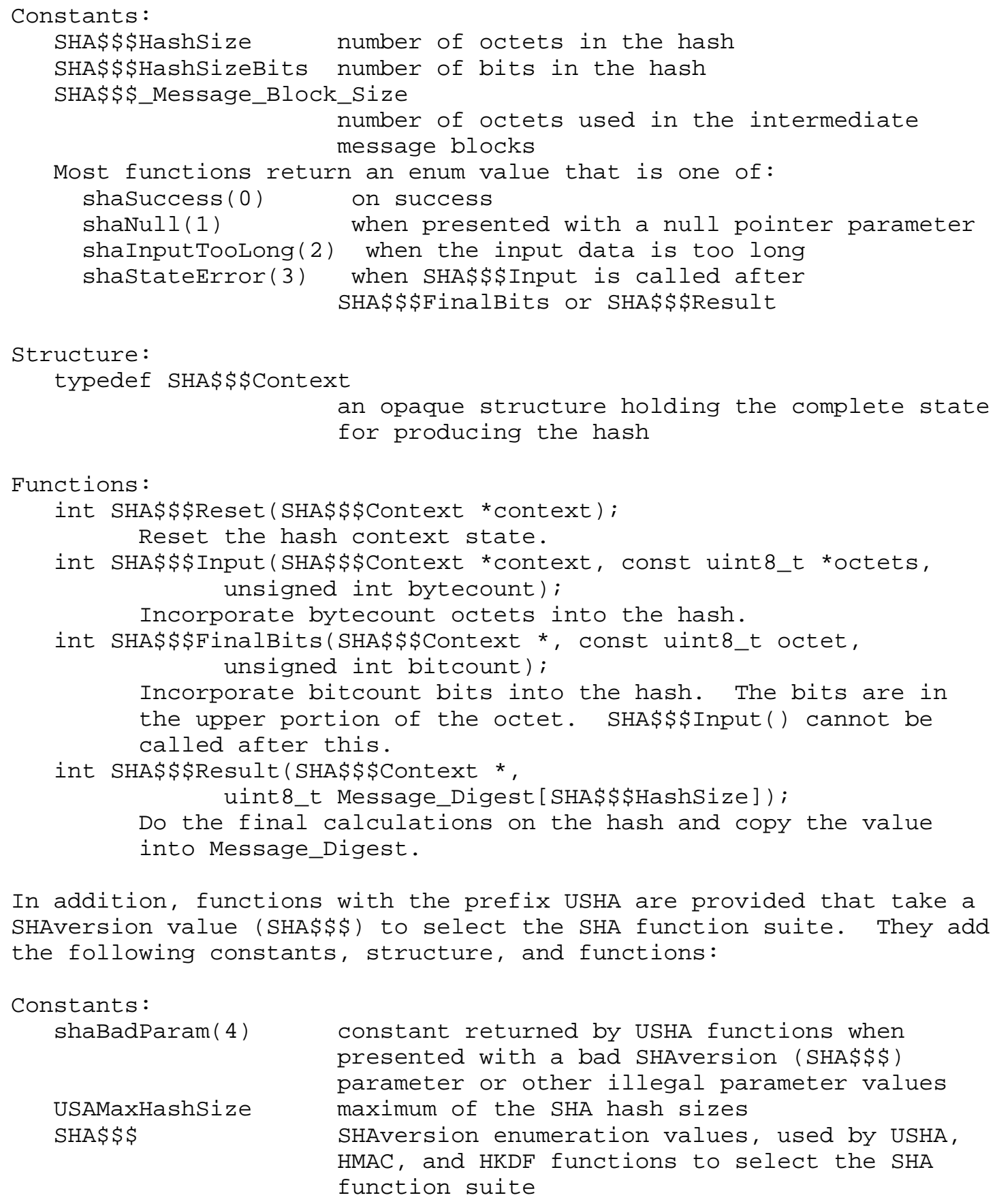

In addition, functions with the prefix USHA are provided that take a SHAversion value (SHA\$\$\$) to select the SHA function suite. They add the following constants, structure, and functions:

Constants:

shaBadParam(4) constant returned by USHA functions when presented with a bad SHAversion (SHA\$\$\$)

USAMaxHashSize parameter or other illegal parameter values maximum of the SHA hash sizes SHA \$ \$ SHAversion enumeration values, used by USHA, HMAC, and HKDF functions to select the SHA function suite 


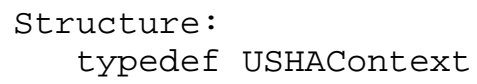

The HMAC functions follow the same pattern to allow any length of text input to be used.

\section{Structure:}

typedef HMACContext an opaque structure holding the complete state for producing the keyed message digest (MAC)

Functions:

int hmacReset (HMACContext *ctx, enum SHAversion whichSha, const unsigned char *key, int key_len);

Reset the MAC context state.

int hmacInput (HMACContext *ctx, const unsigned char *text, int text_len);

Incorporate text_len octets into the MAC.

int hmacFinalBits (HMACContext * ctx, const uint8_t bits, unsigned int bitcount);

Incorporate bitcount bits into the MAC.

int hmacResult (HMACContext *ctx, uint8_t Message_Digest[USHAMaxHashSize]); 
Do the final calculations on the MAC and copy the value into Message_Digest. Octets in Message_Digest beyond

USHAHashSize(whichSha) are left untouched.

In addition, a combined interface is provided, similar to that shown

in [RFC2104], that allows a fixed-length text input to be used.

int hmac(SHAversion whichsha,

const unsigned char *text, int text_len,

const unsigned char *key, int key_len,

uint8_t Message_Digest[USHAMaxHashSize]);

Calculate the given digest for the given text and key, and

return the resulting MAC. Octets in Message_Digest beyond

USHAHashSize(whichSha) are left untouched.

The HKDF functions follow the same pattern to allow any length of text input to be used.

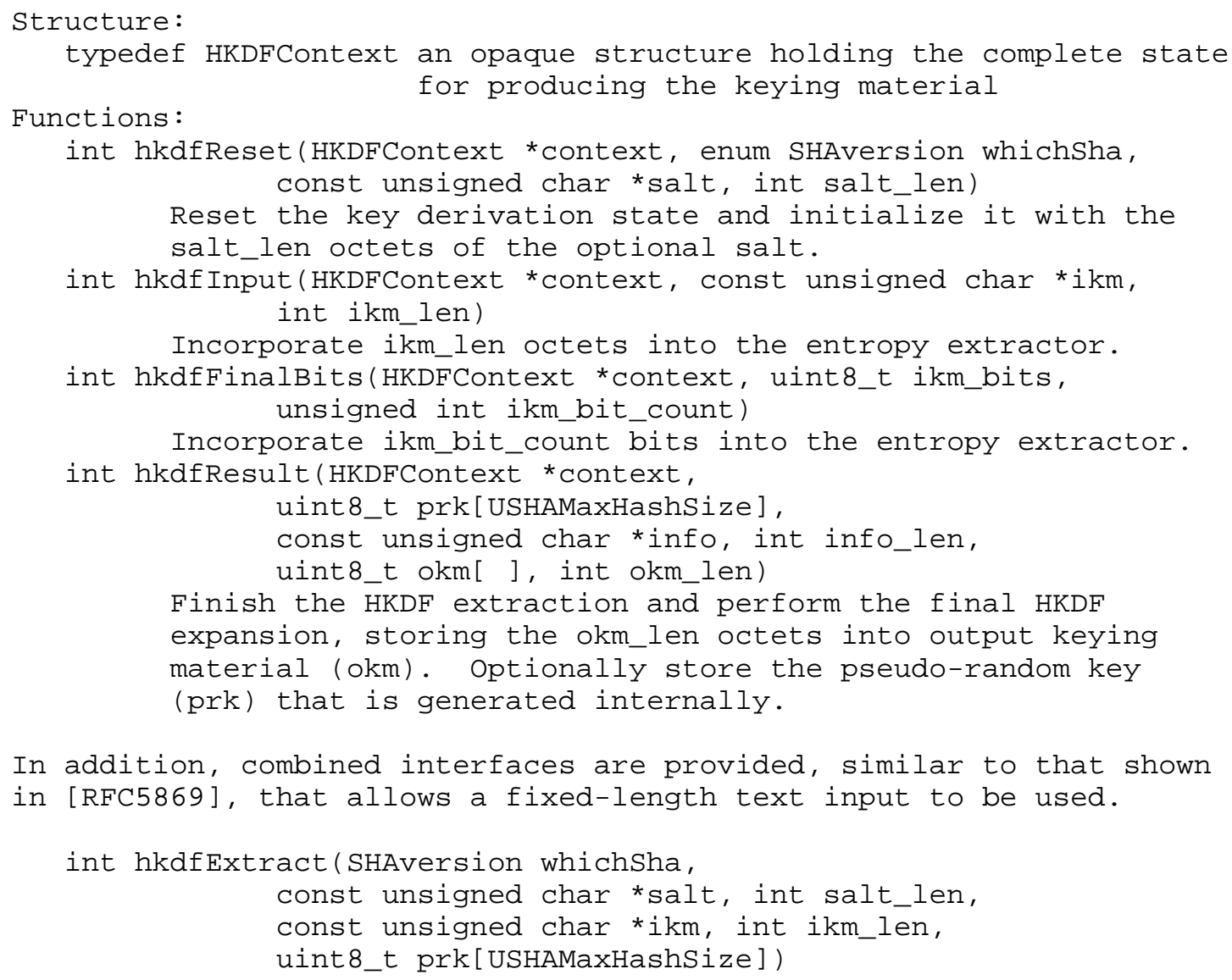


Perform HKDF extraction, combining the salt_len octets of the optional salt with the ikm_len octets of the input keying material (i km) to form the pseudo-random key prk. The output prk must be large enough to hold the octets appropriate for the given hash type.

int hkdfExpand(SHAversion whichSha, const uint8_t prk[ ], int prk_len, const unsigned char *info, int info_len, uint8_t okm[ ], int okm_len)

Perform HKDF expansion, combining the prk_len octets of the pseudo-random key prk with the info_len octets of info to form the okm_len octets stored in okm.

int hkdf (SHAversion whichSha,

const unsigned char *salt, int salt_len, const unsigned char *ikm, int ikm_len, const unsigned char *info, int info_len, uint8_t okm[ ], int okm_len)

This combined interface performs both HKDF extraction and expansion. The variables are the same as in hkdfExtract() and hkdfExpand ().

8.1. The Header Files

8.1.1. The .h file

The following sha.h file, as stated in the comments within the file, assumes that <stdint.h> is available on your system. If it is not, you should change to including <stdint-example.h>, provided in Section 8.1.2, or the like.

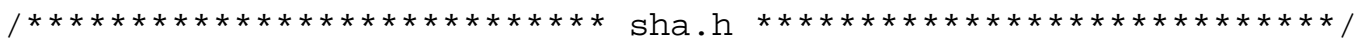

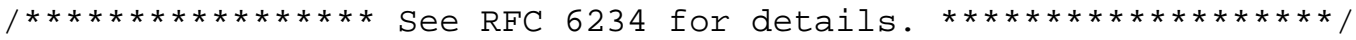

$/ *$

Copyright (c) 2011 IETF Trust and the persons identified as authors of the code. All rights reserved.

Redistribution and use in source and binary forms, with or without modification, are permitted provided that the following conditions are met:

- Redistributions of source code must retain the above copyright notice, this list of conditions and the following disclaimer. 
- Redistributions in binary form must reproduce the above copyright notice, this list of conditions and the following disclaimer in the documentation and/or other materials provided with the distribution.

- Neither the name of Internet Society, IETF or IETF Trust, nor the names of specific contributors, may be used to endorse or promote products derived from this software without specific prior written permission.

THIS SOFTWARE IS PROVIDED BY THE COPYRIGHT HOLDERS AND CONTRIBUTORS "AS IS" AND ANY EXPRESS OR IMPLIED WARRANTIES, INCLUDING, BUT NOT LIMITED TO, THE IMPLIED WARRANTIES OF MERCHANTABILITY AND FITNESS FOR A PARTICULAR PURPOSE ARE DISCLAIMED. IN NO EVENT SHALL THE COPYRIGHT OWNER OR CONTRIBUTORS BE LIABLE FOR ANY DIRECT, INDIRECT, INCIDENTAL, SPECIAL, EXEMPLARY, OR CONSEQUENTIAL DAMAGES (INCLUDING, BUT NOT LIMITED TO, PROCUREMENT OF SUBSTITUTE GOODS OR SERVICES; LOSS OF USE, DATA, OR PROFITS; OR BUSINESS INTERRUPTION) HOWEVER CAUSED AND ON ANY THEORY OF LIABILITY, WHETHER IN CONTRACT, STRICT LIABILITY, OR TORT (INCLUDING NEGLIGENCE OR OTHERWISE) ARISING IN ANY WAY OUT OF THE USE OF THIS SOFTWARE, * / EVEN IF ADVISED OF THE POSSIBILITY OF SUCH DAMAGE.

\#ifndef _.SHA_H_ \#define _SHA_H_

/

* Description:

* This file implements the Secure Hash Algorithms

* as defined in the U.S. National Institute of Standards

* and Technology Federal Information Processing Standards

* Publication (FIPS PUB) 180-3 published in October 2008

* and formerly defined in its predecessors, FIPS PUB 180-1

* and FIP PUB 180-2.

A combined document showing all algorithms is available at http://csrc.nist.gov/publications/fips/ fips180-3/fips180-3_final.pdf

The five hashes are defined in these sizes: SHA-1 20 byte / 160 bit SHA-224 28 byte / 224 bit SHA-256 32 byte / 256 bit SHA-384 48 byte / 384 bit SHA-512 64 byte / 512 bit 


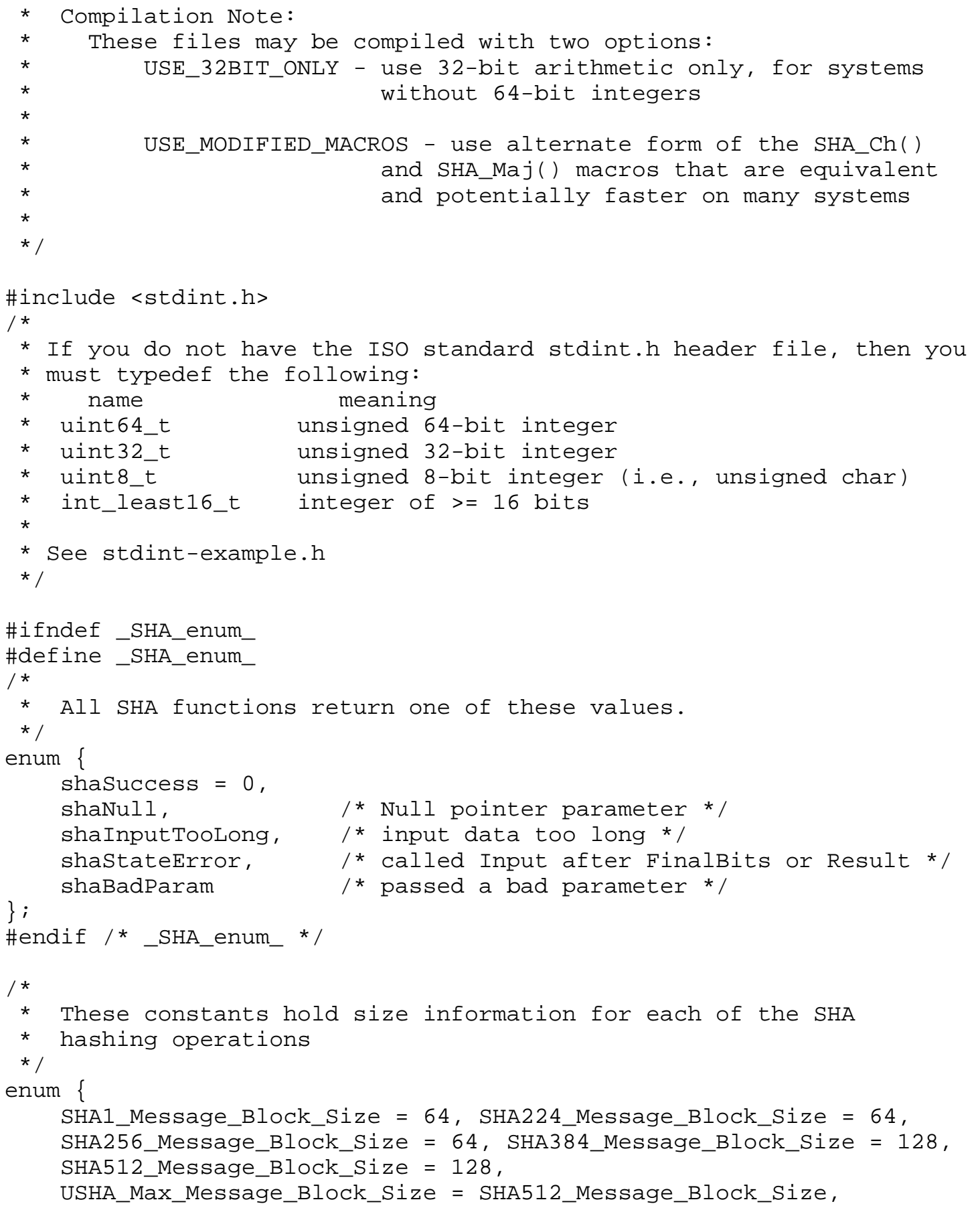




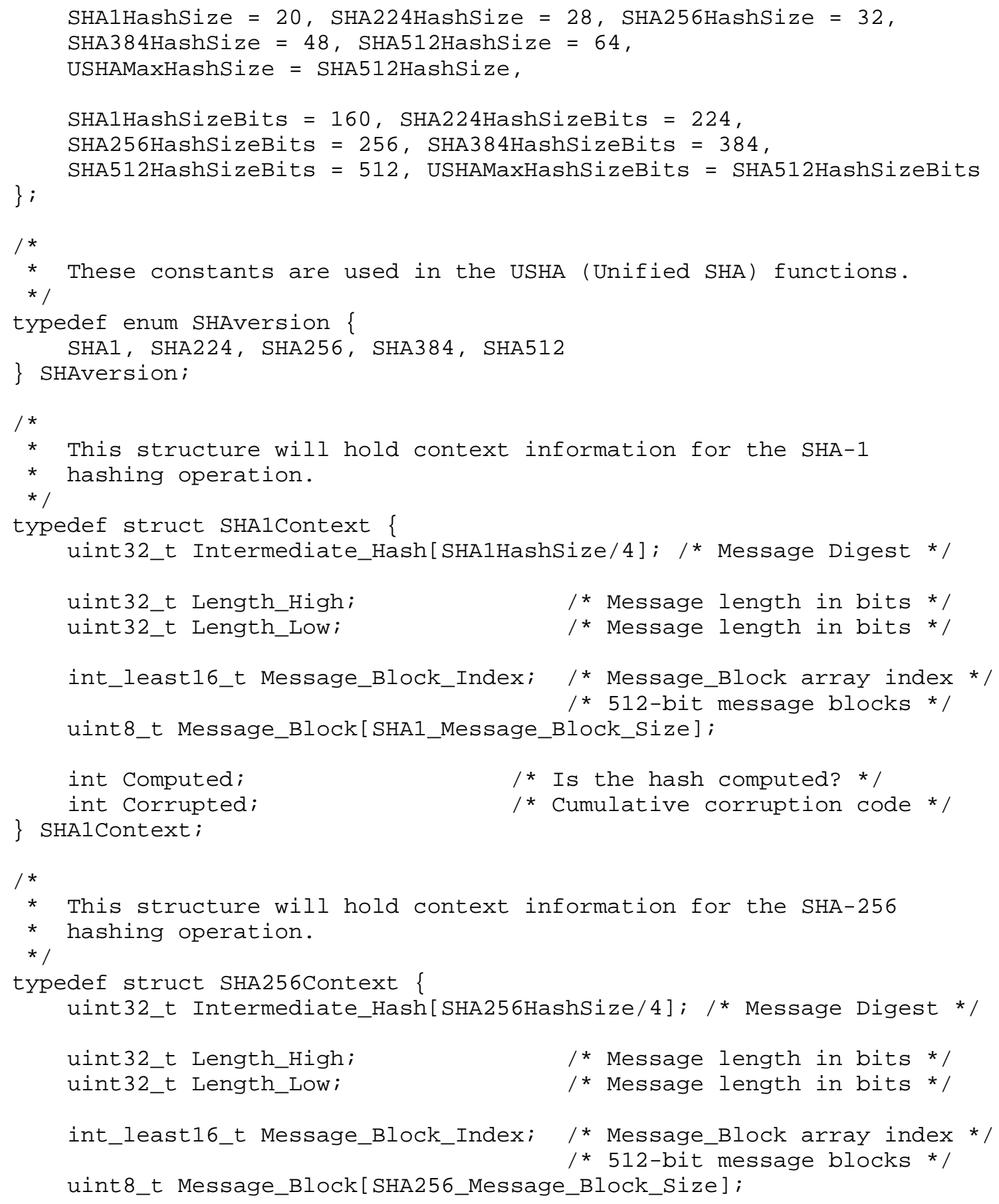




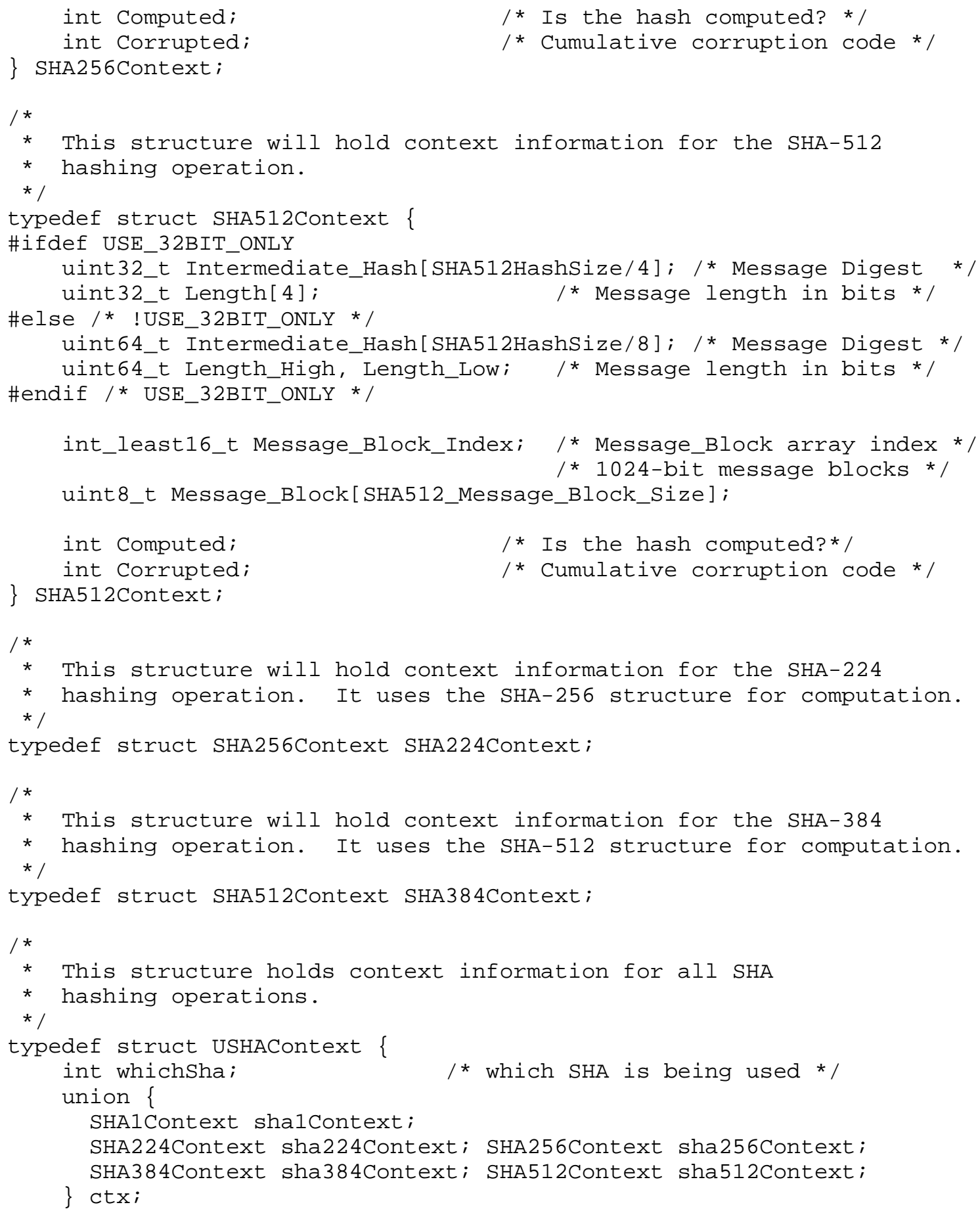


\} USHAContext ;

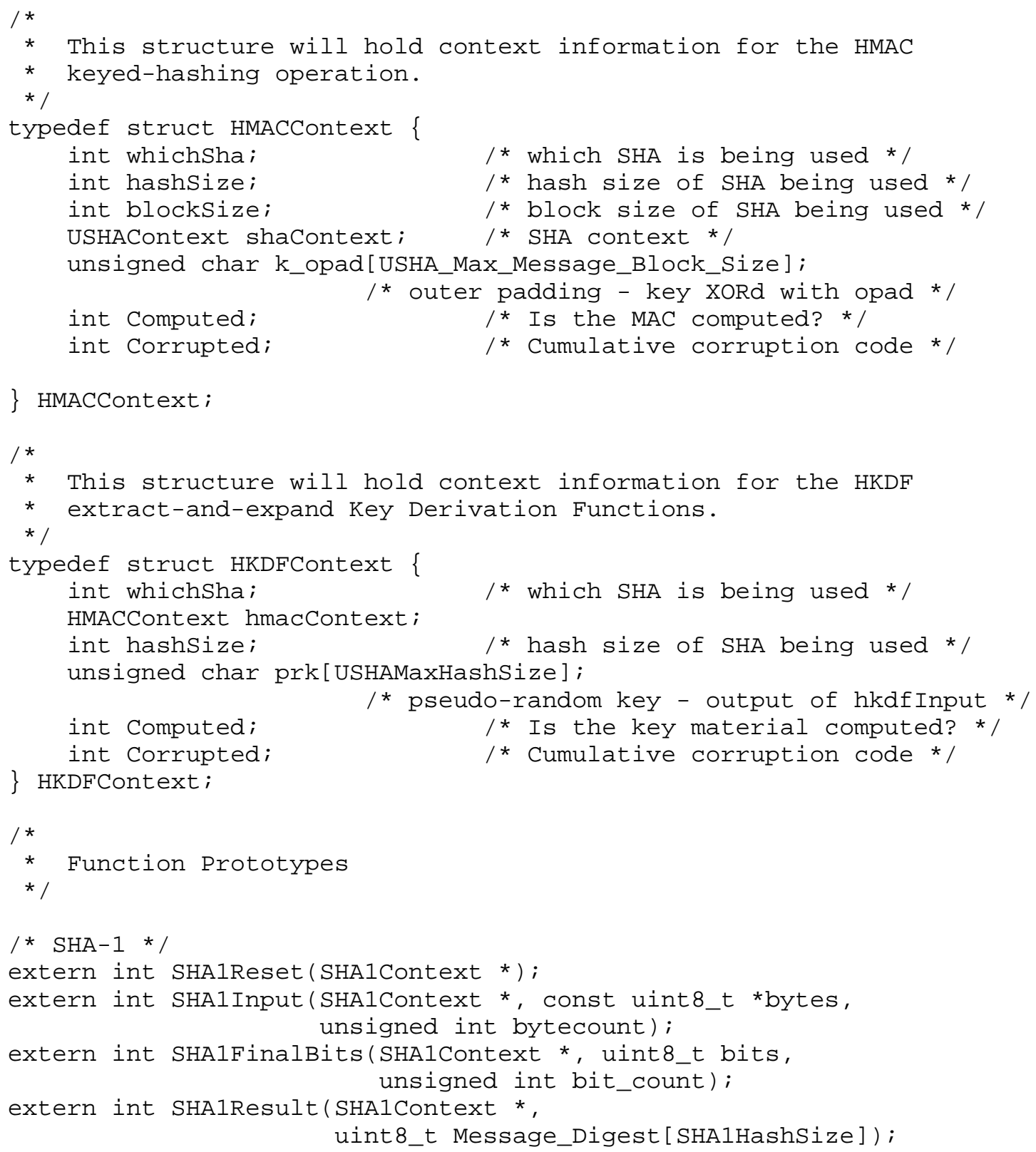




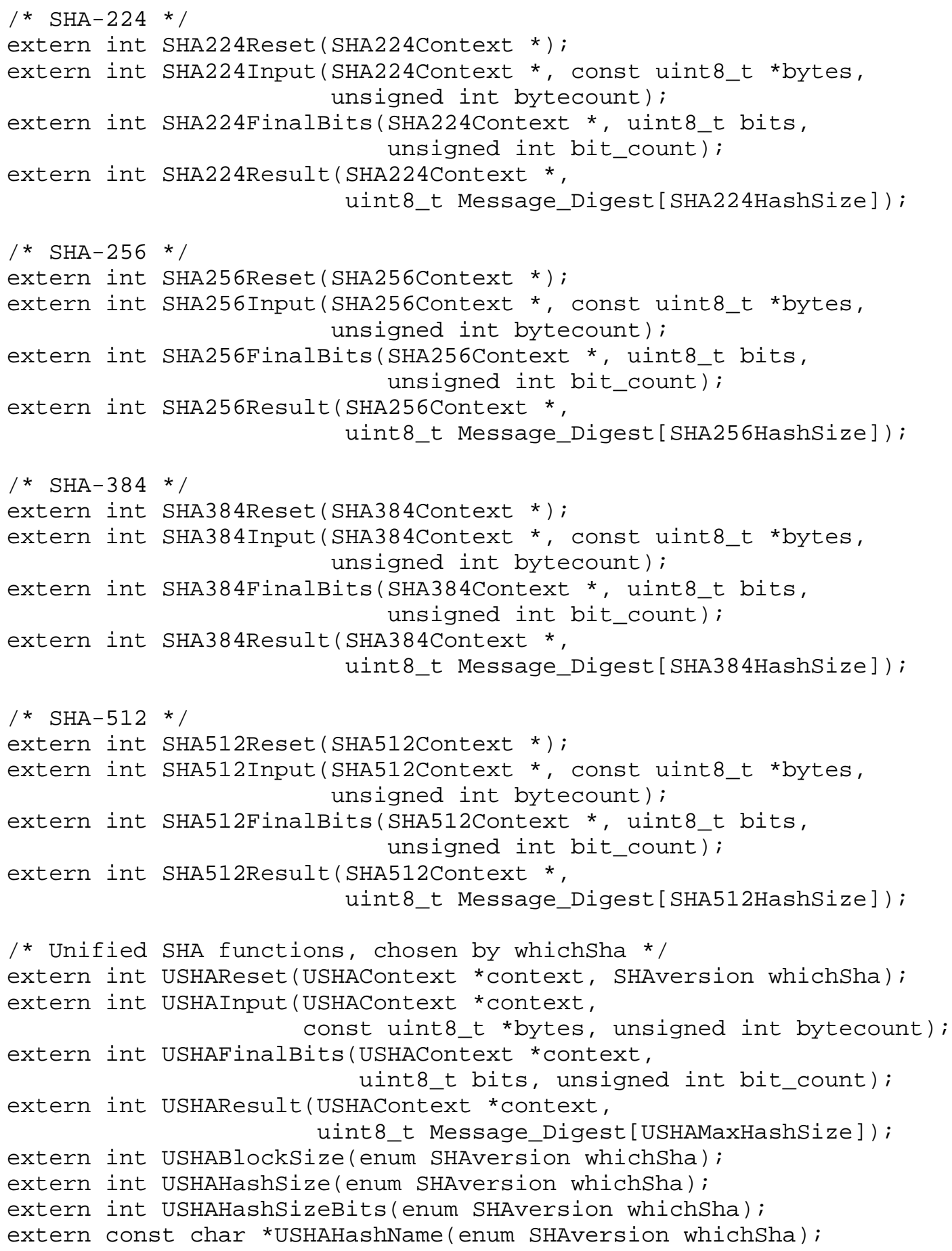




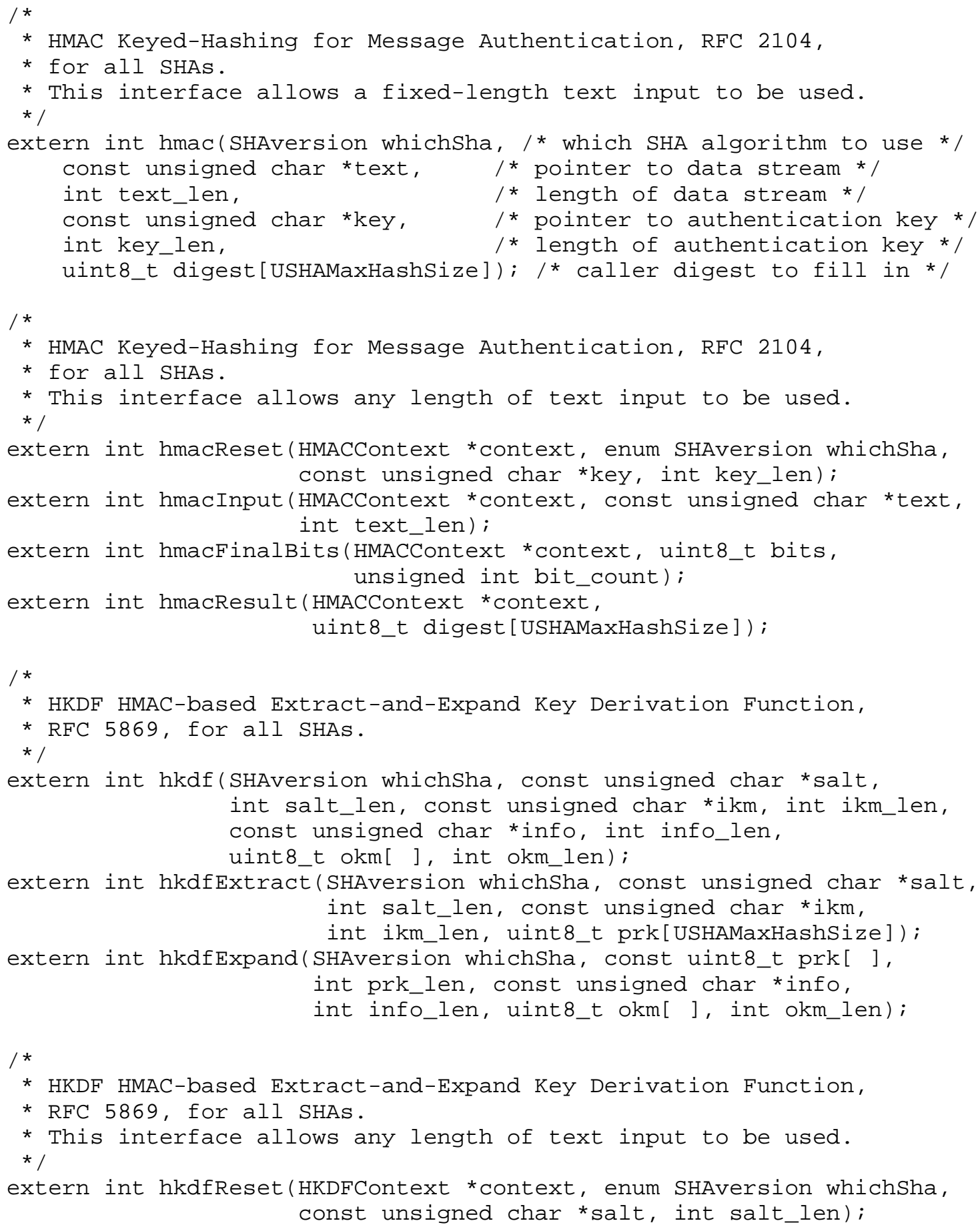




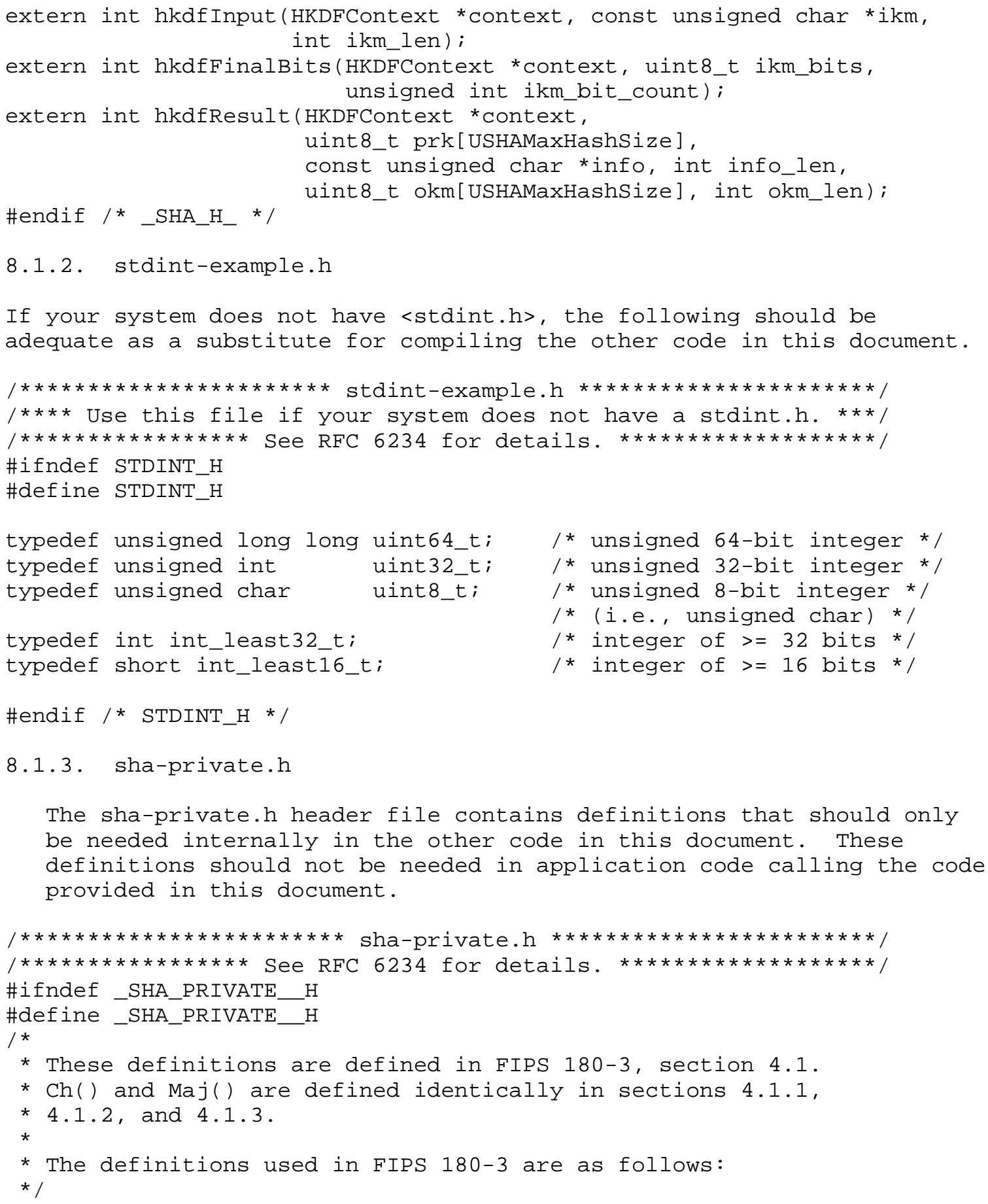




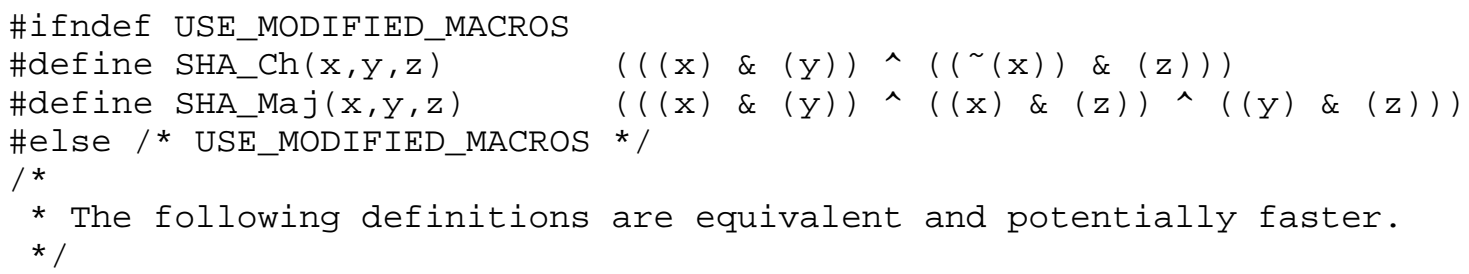




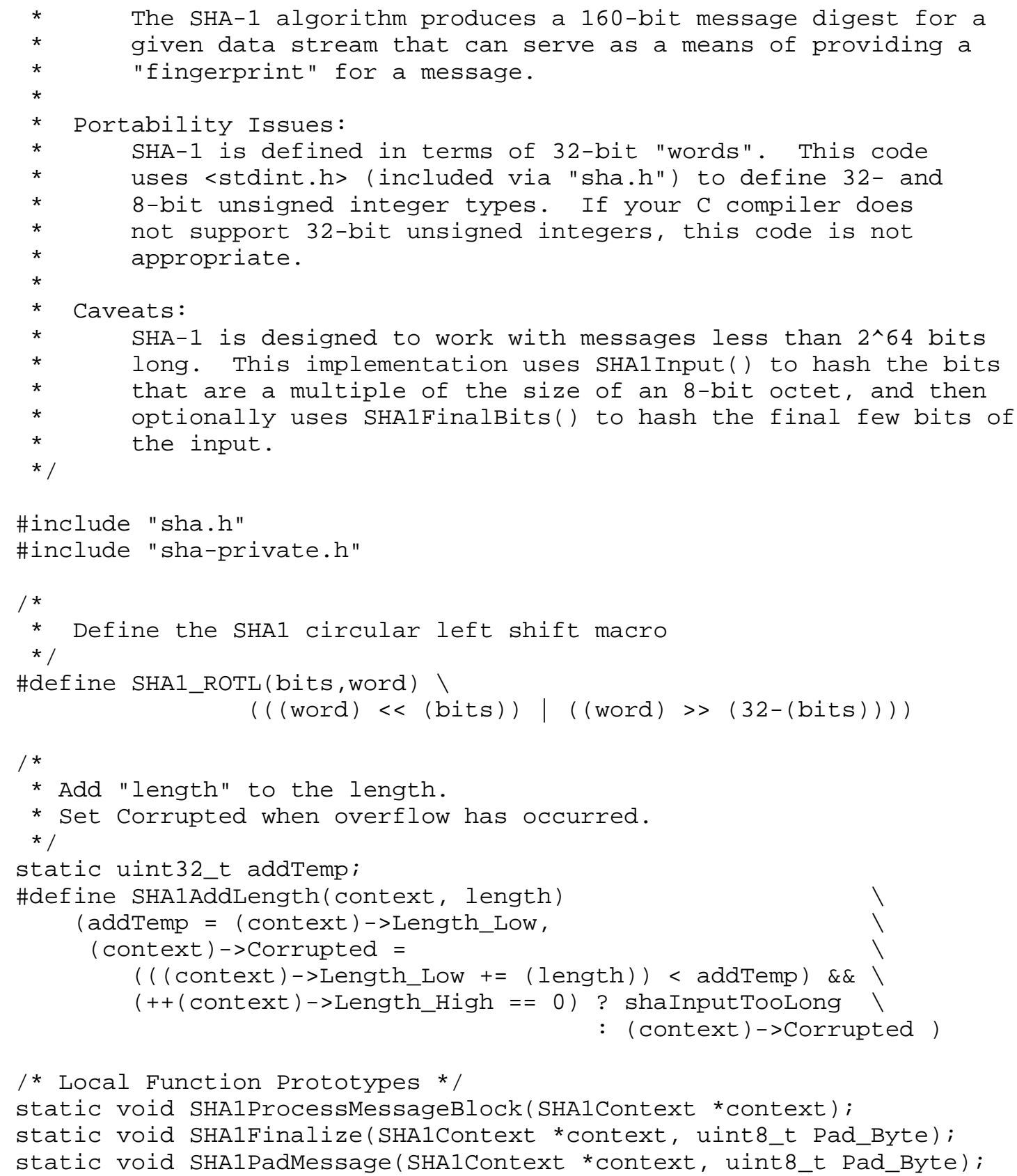




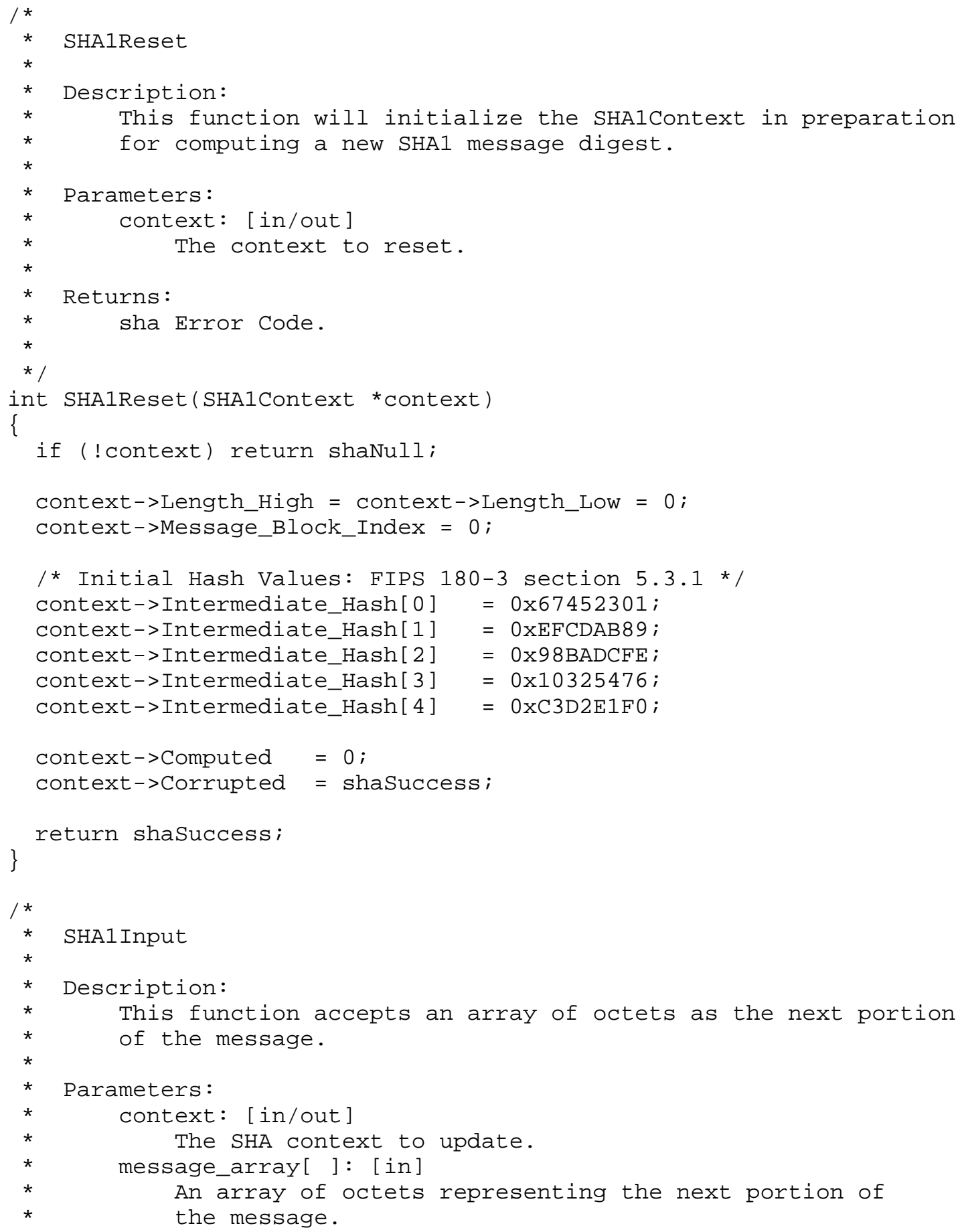




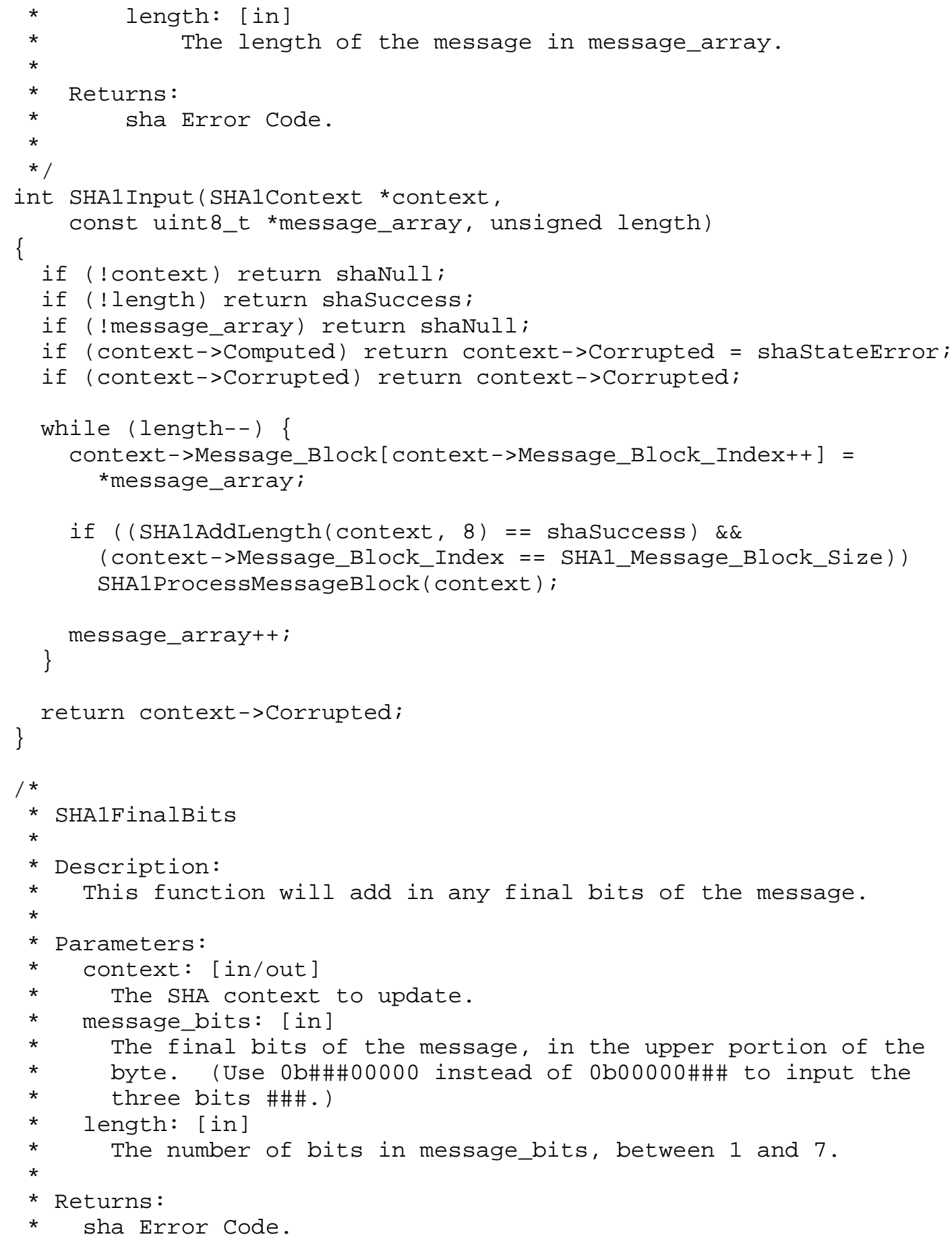




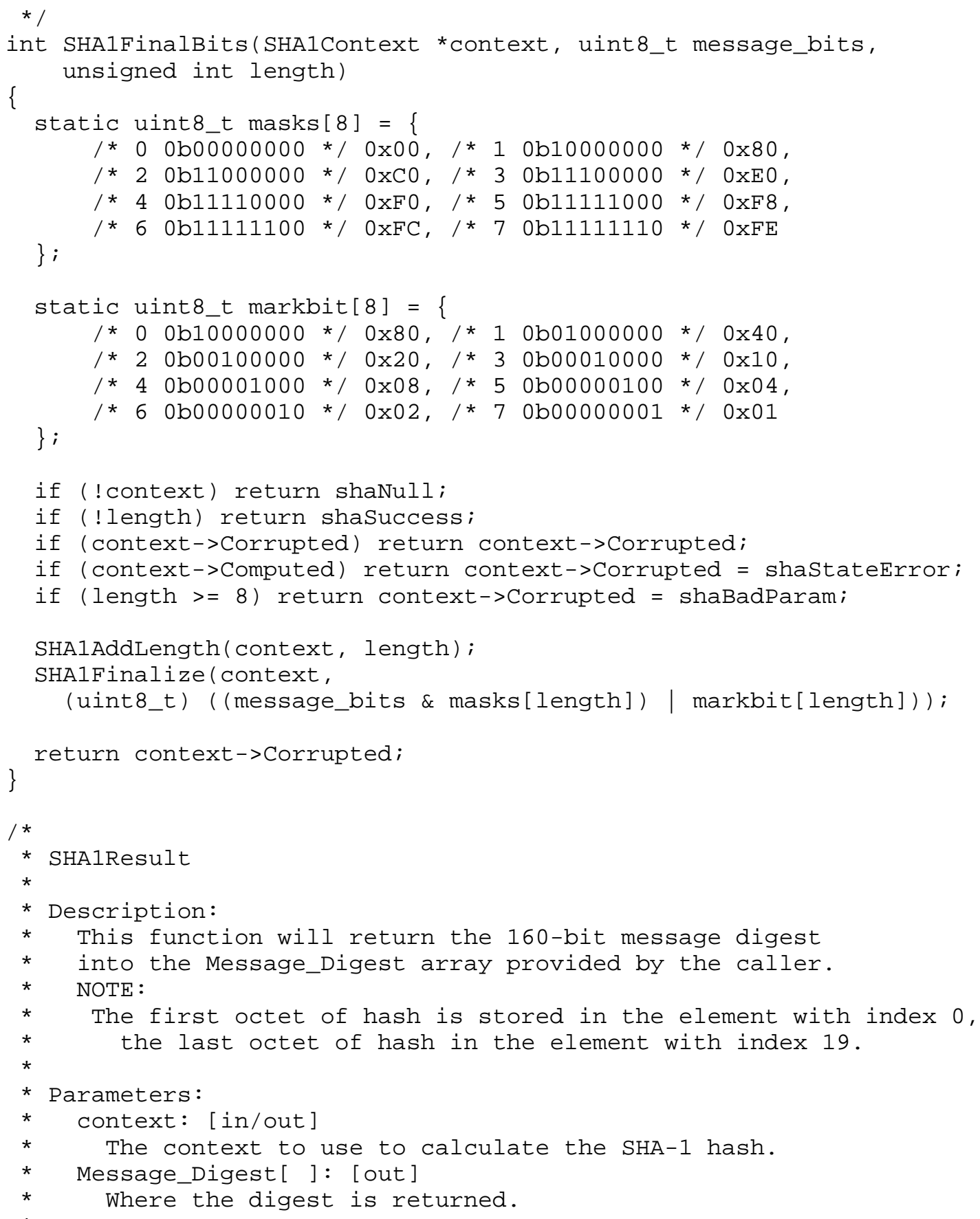




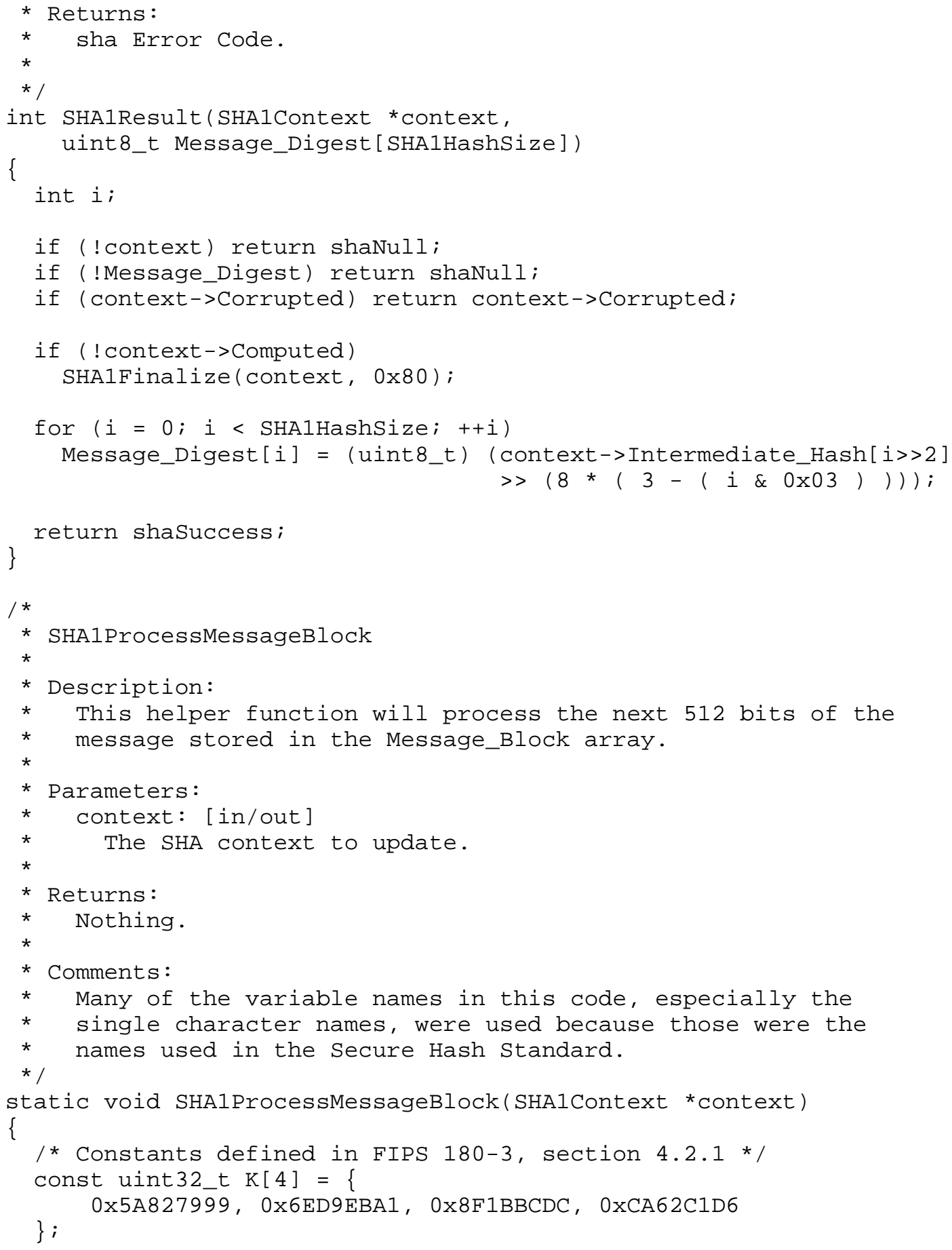




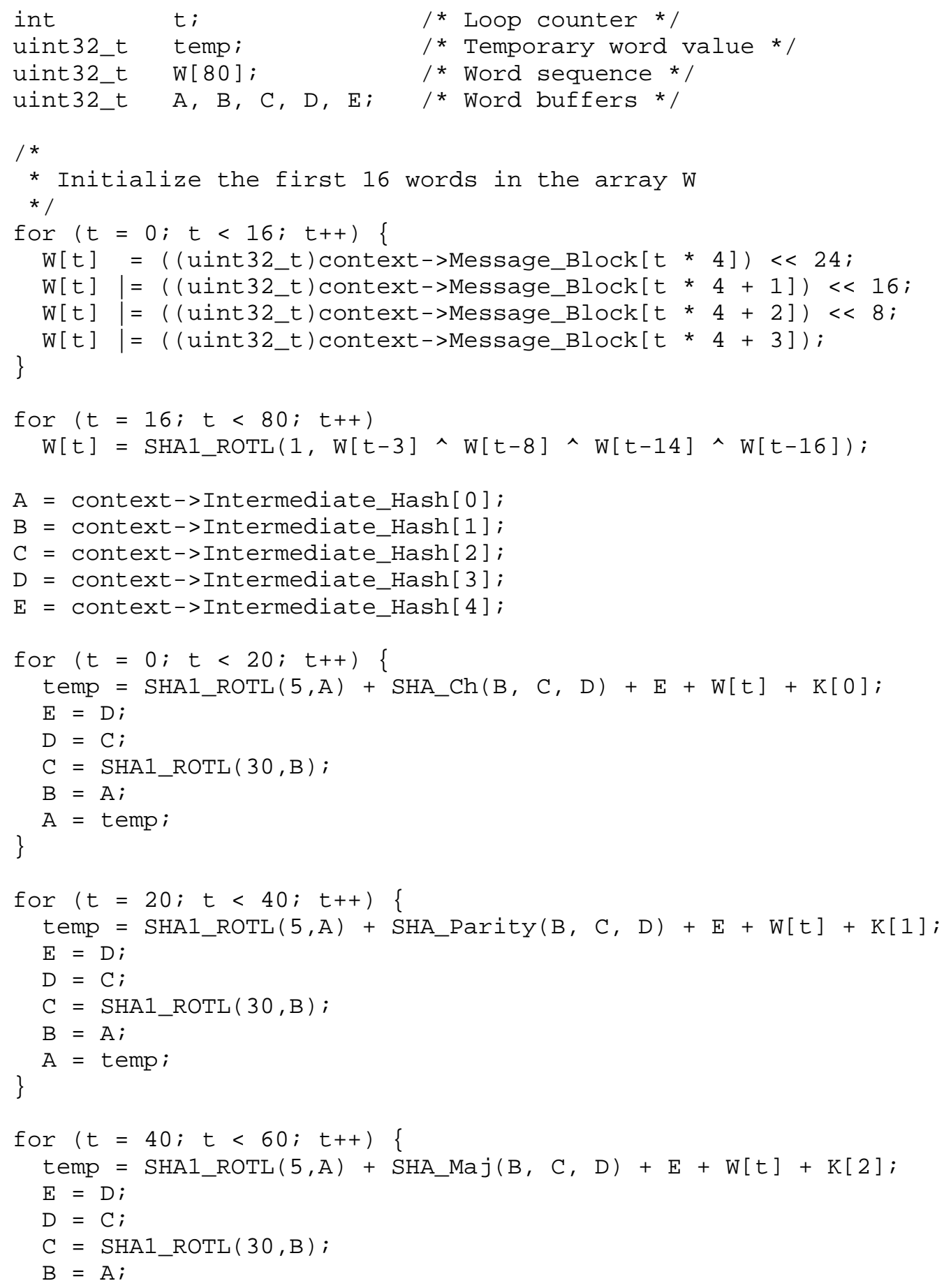




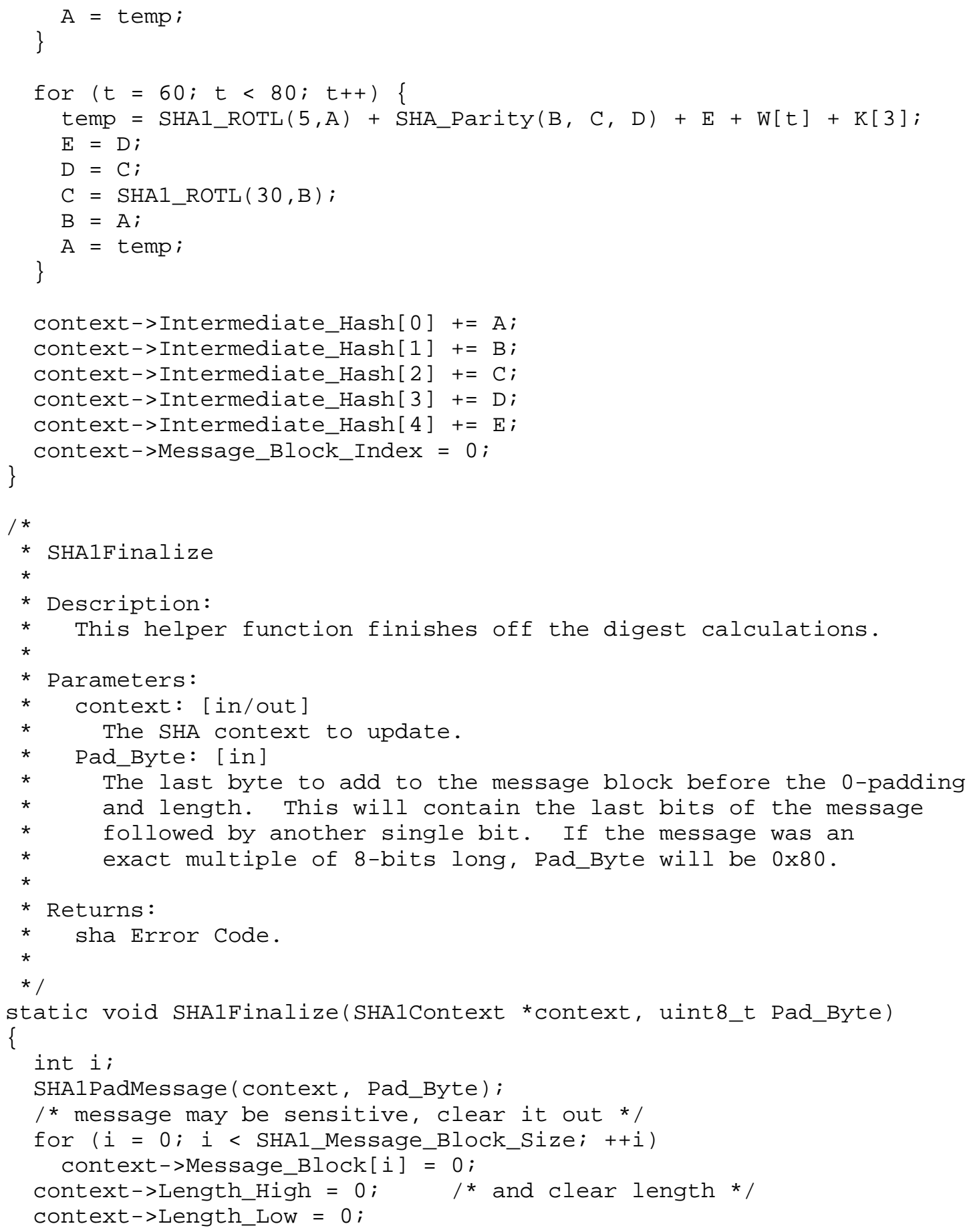




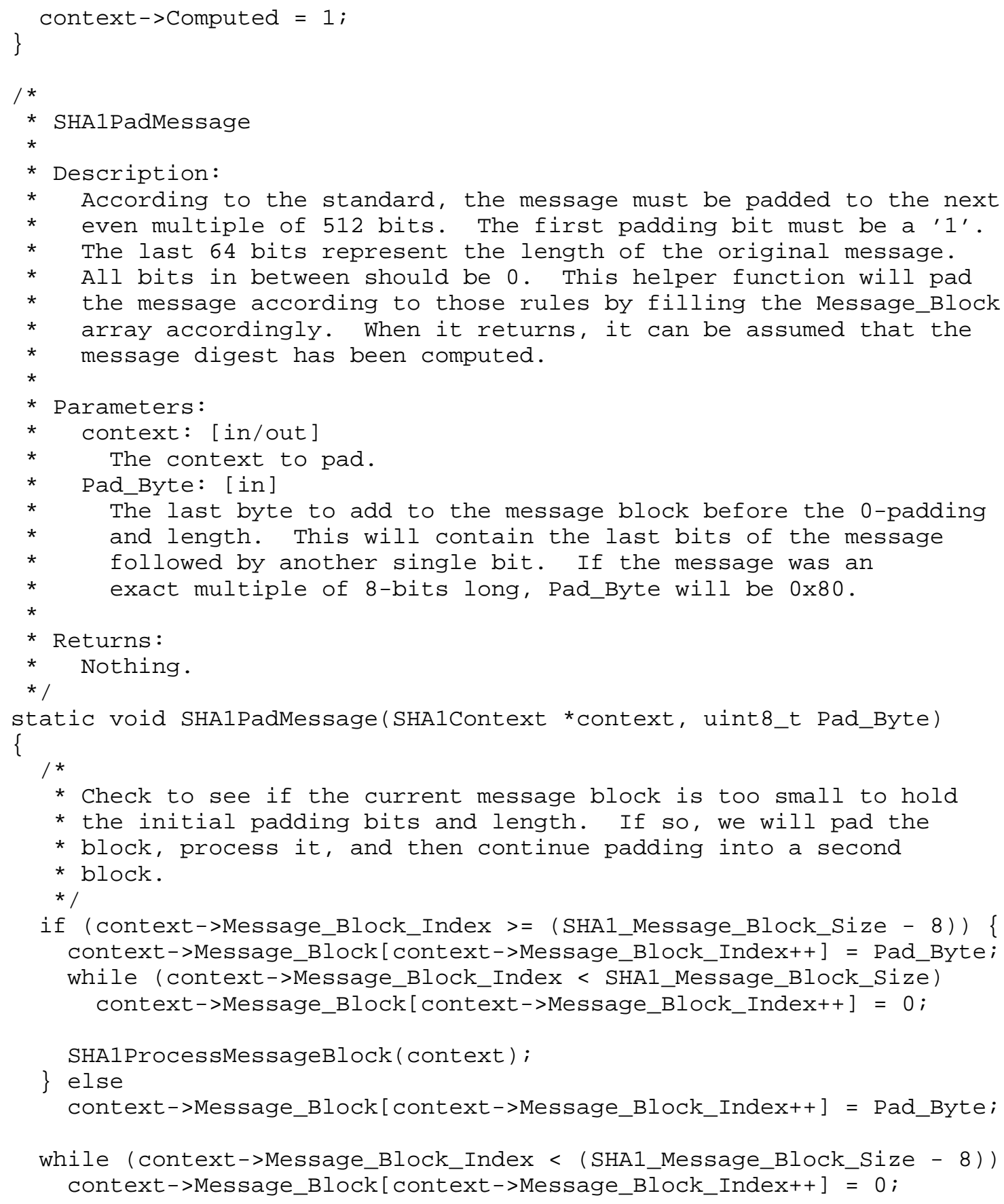




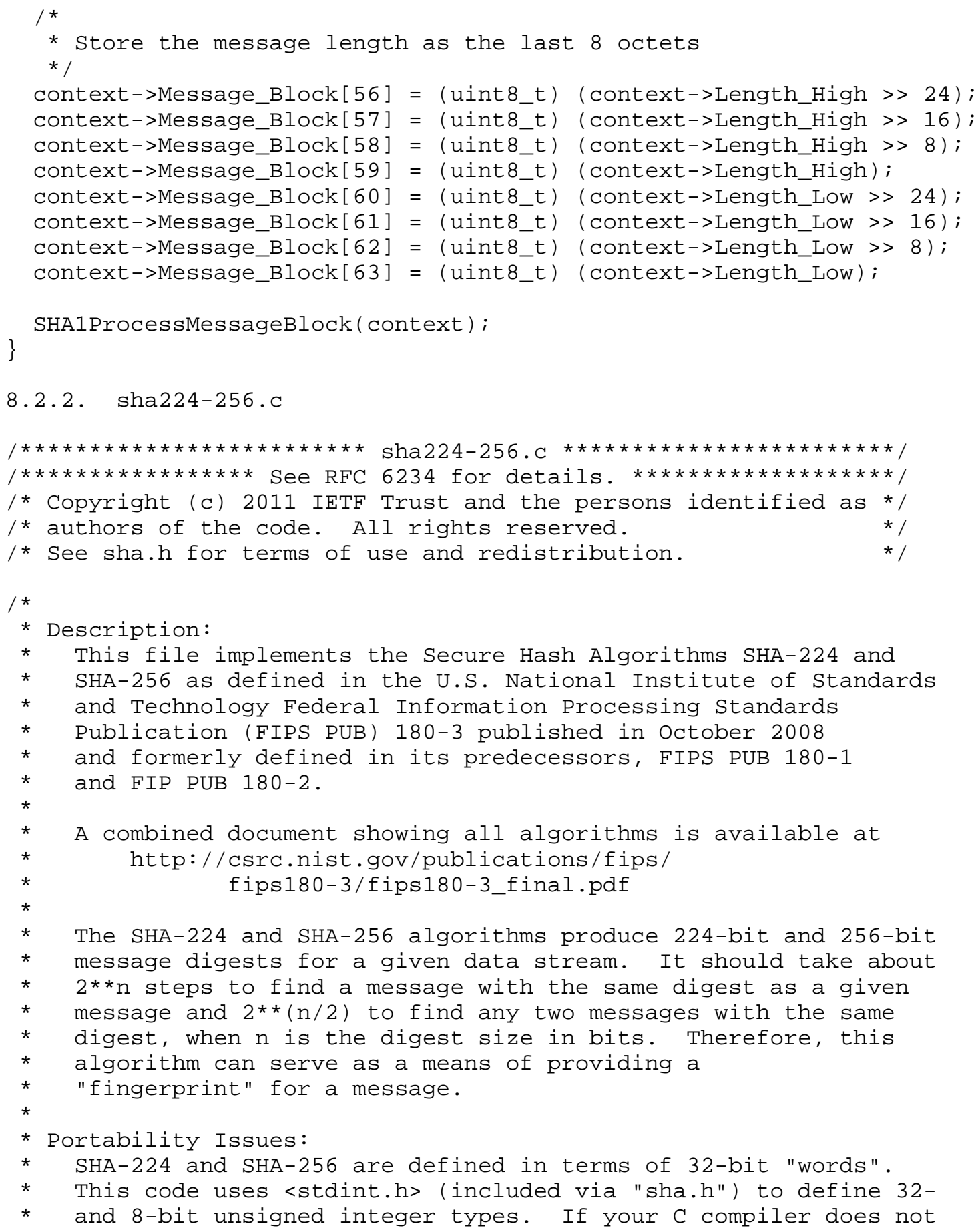




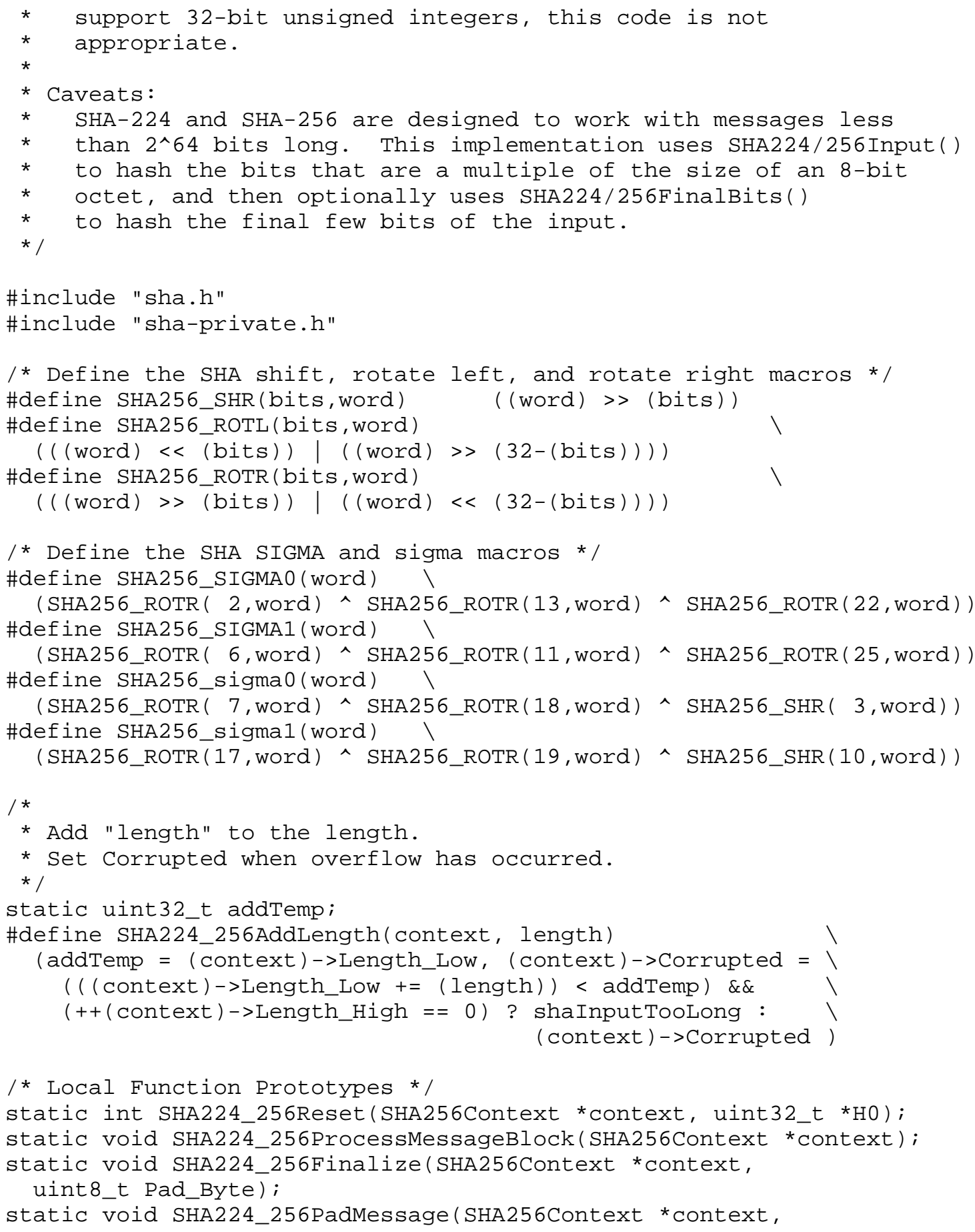




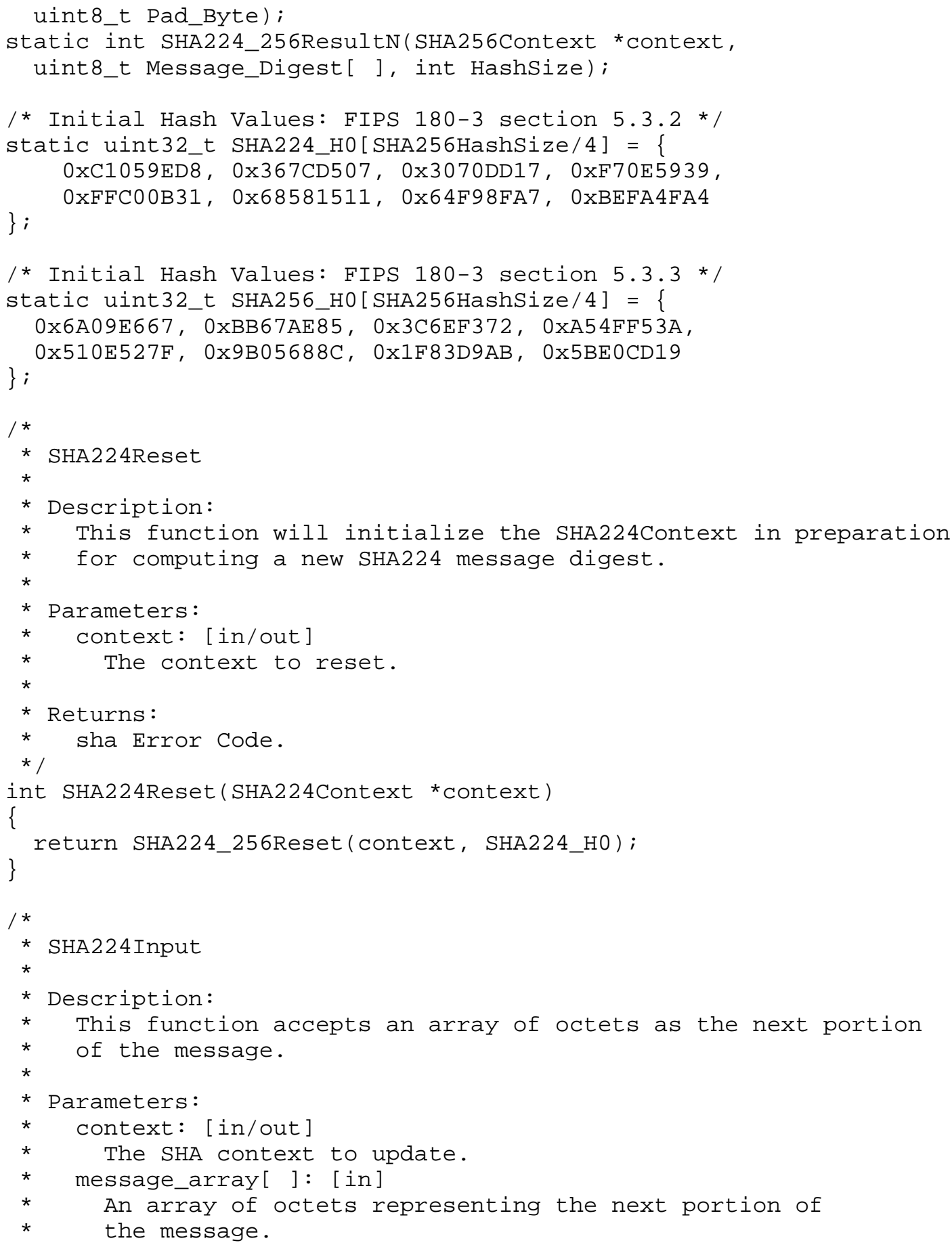




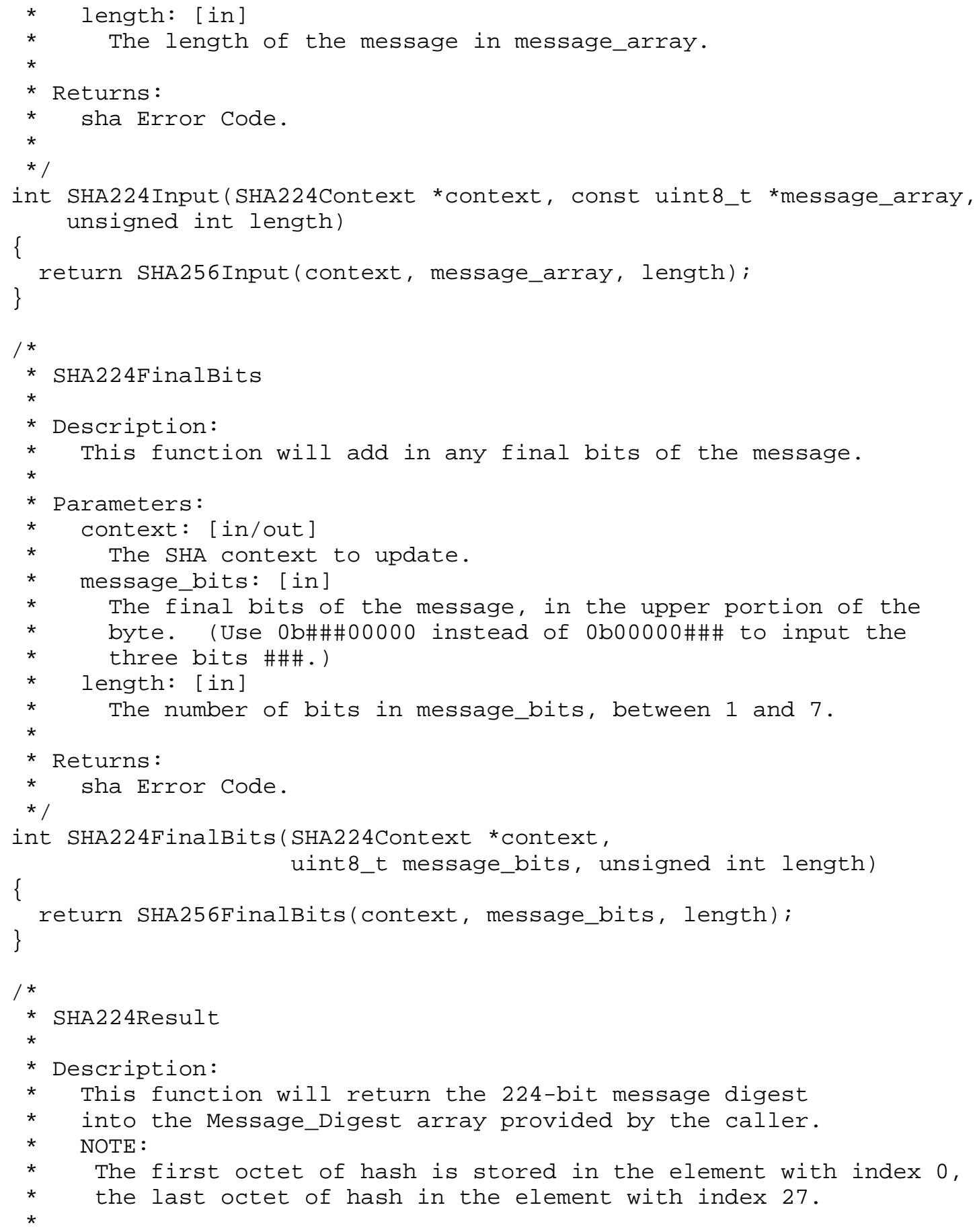




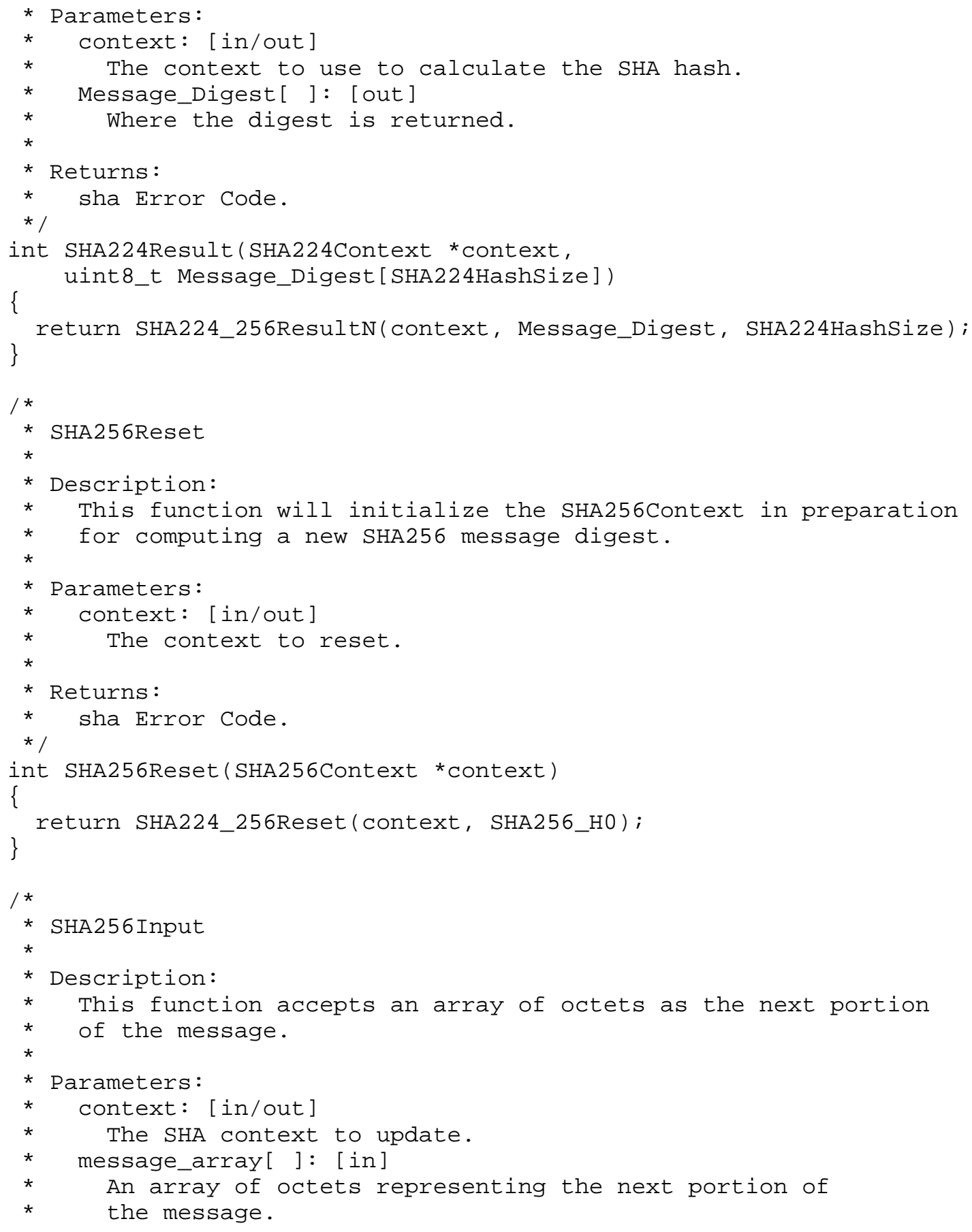




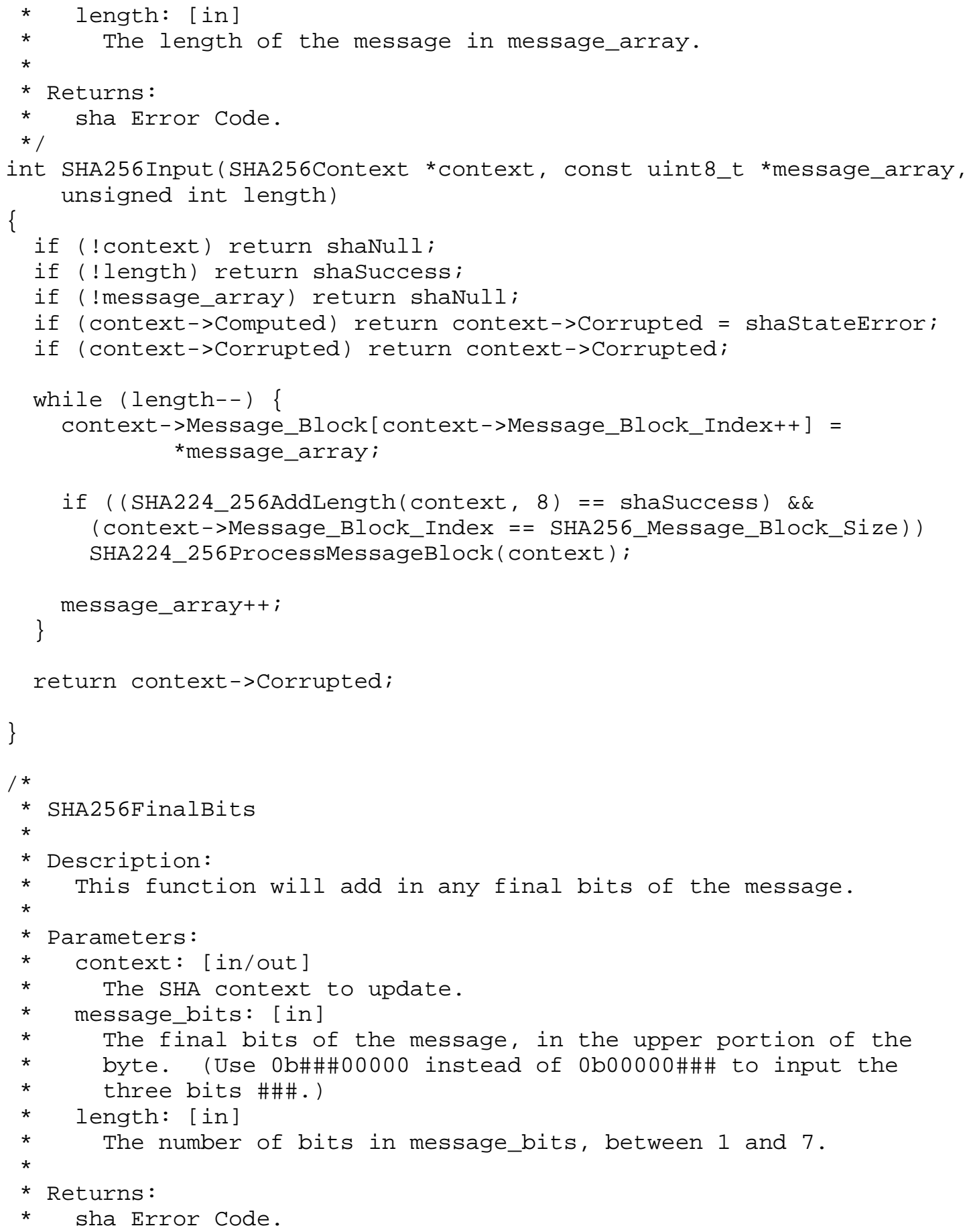




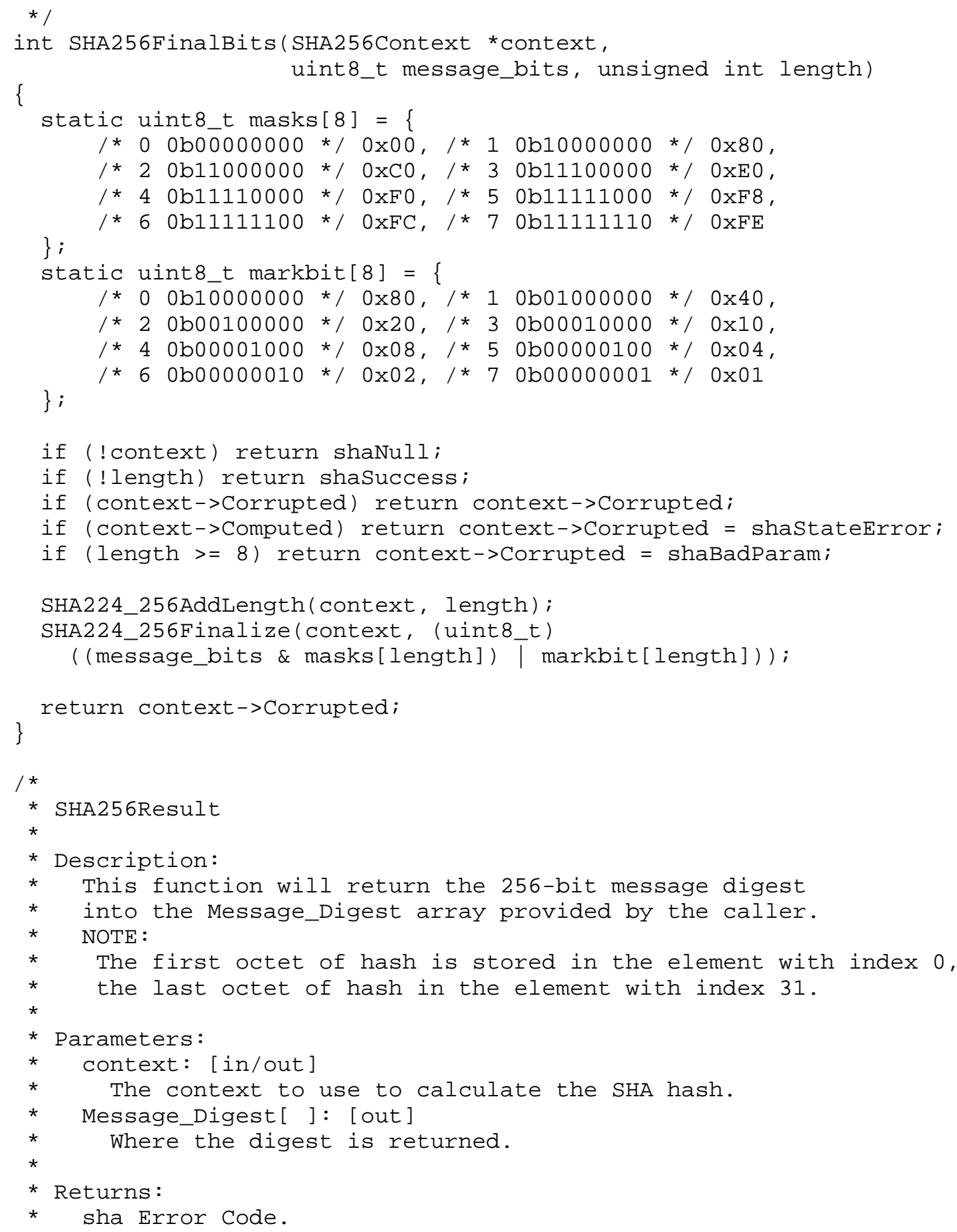




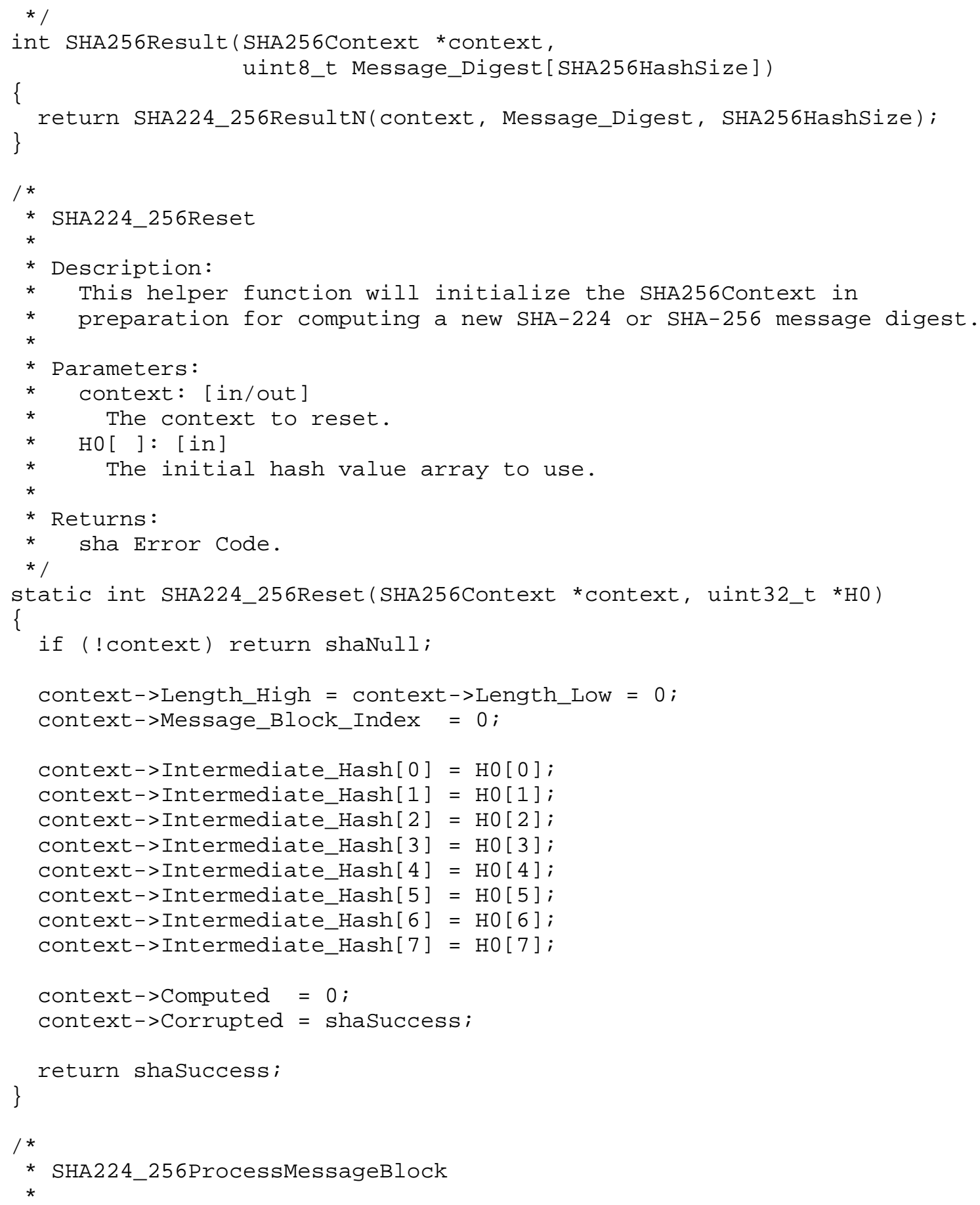




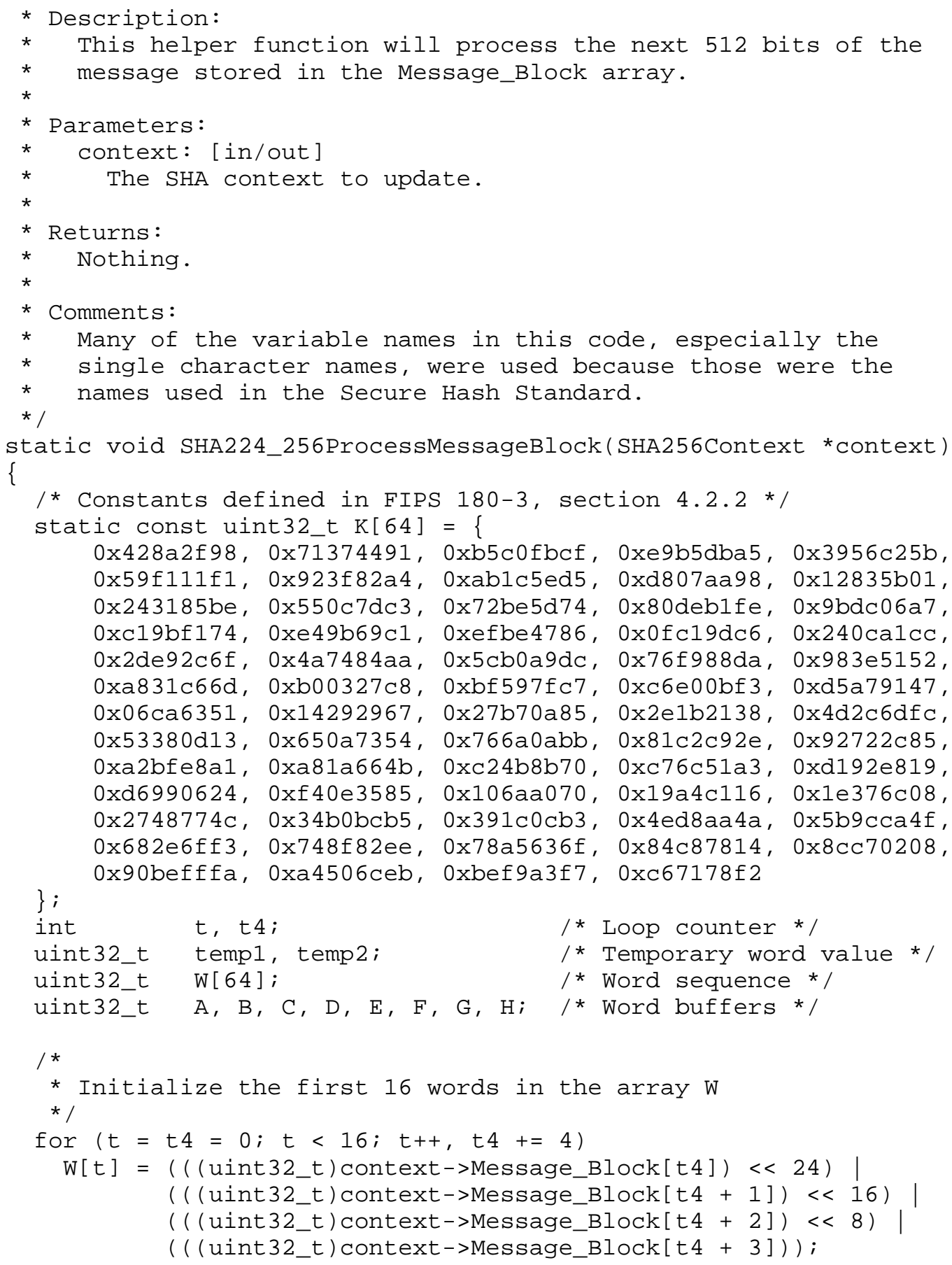




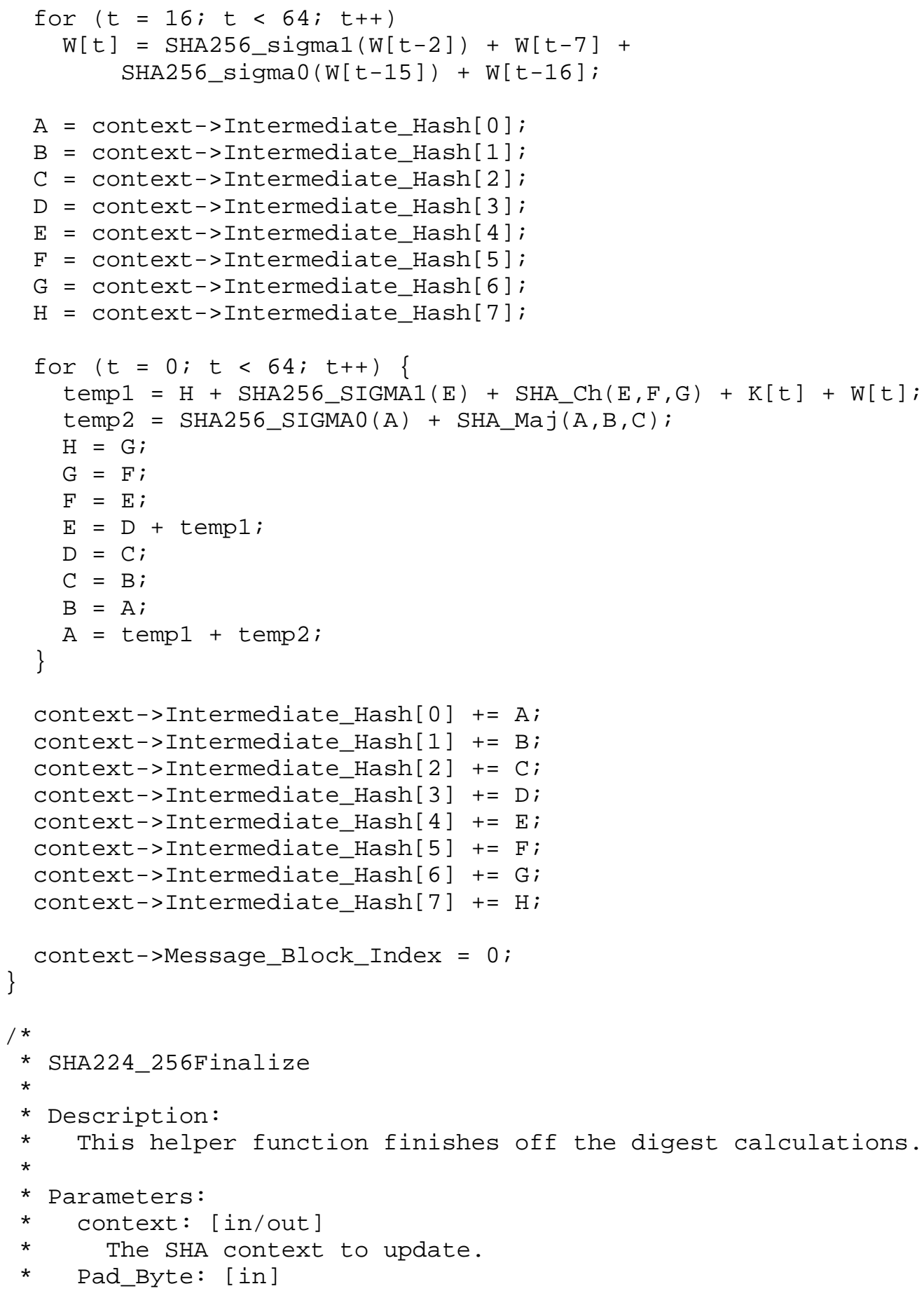




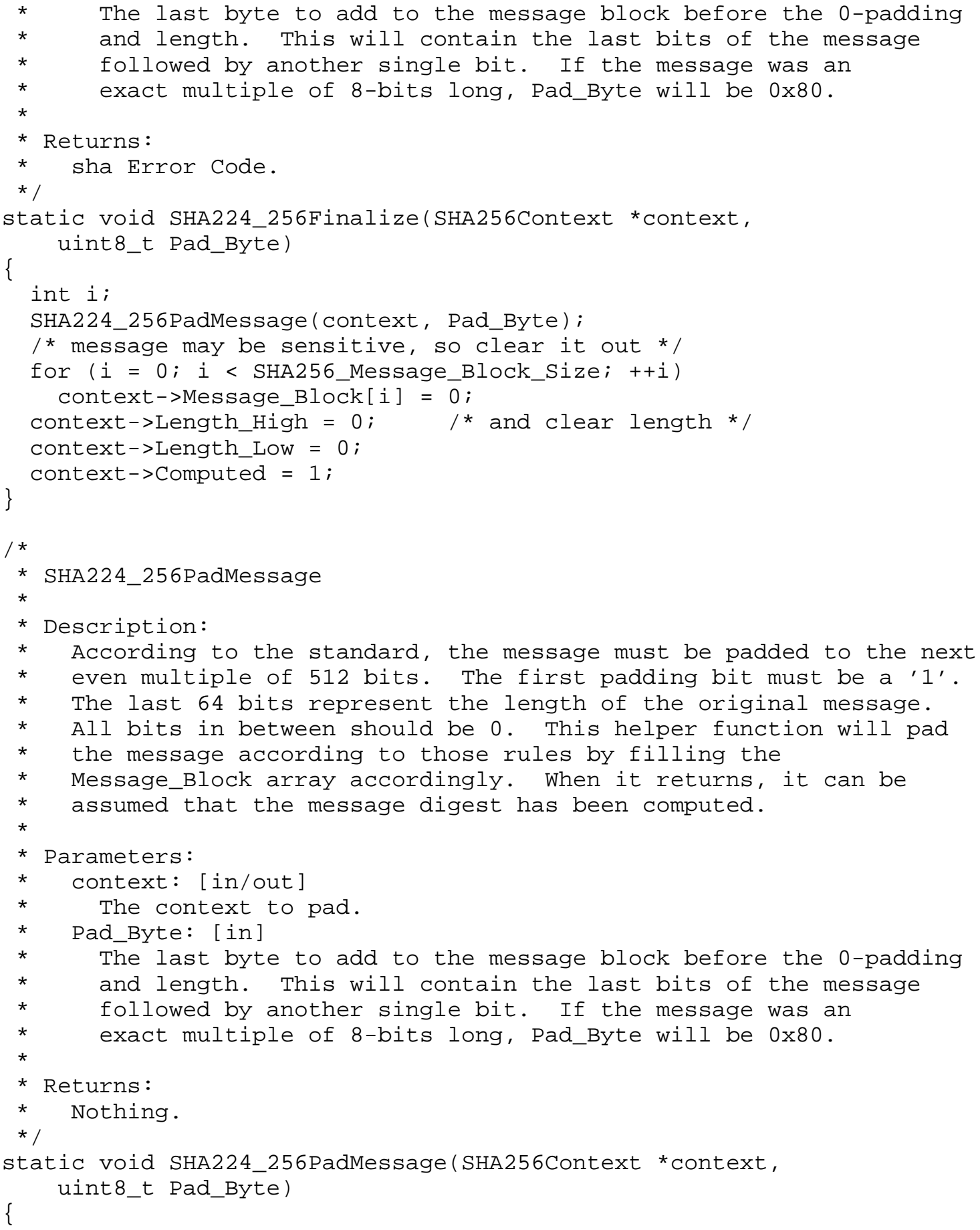




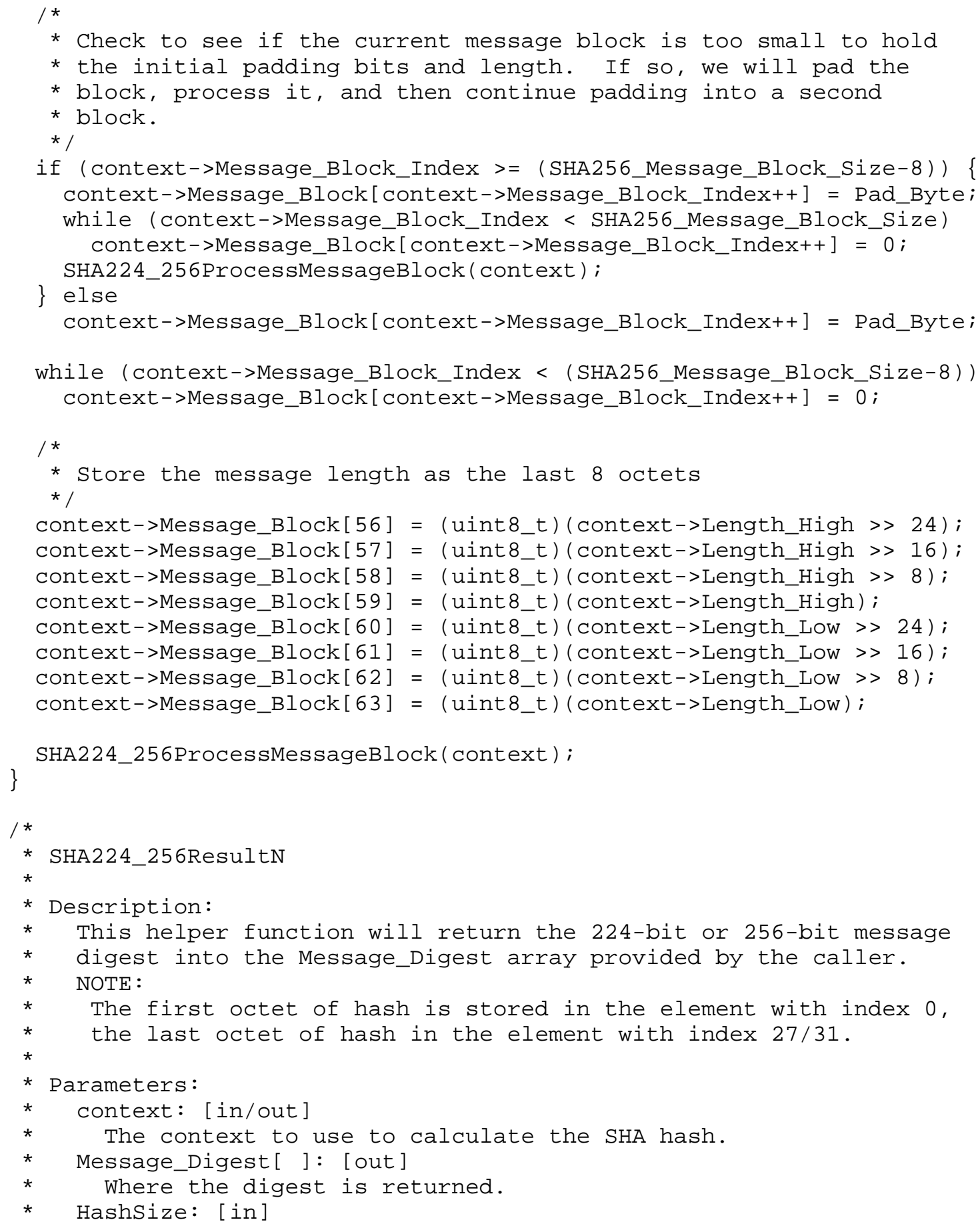




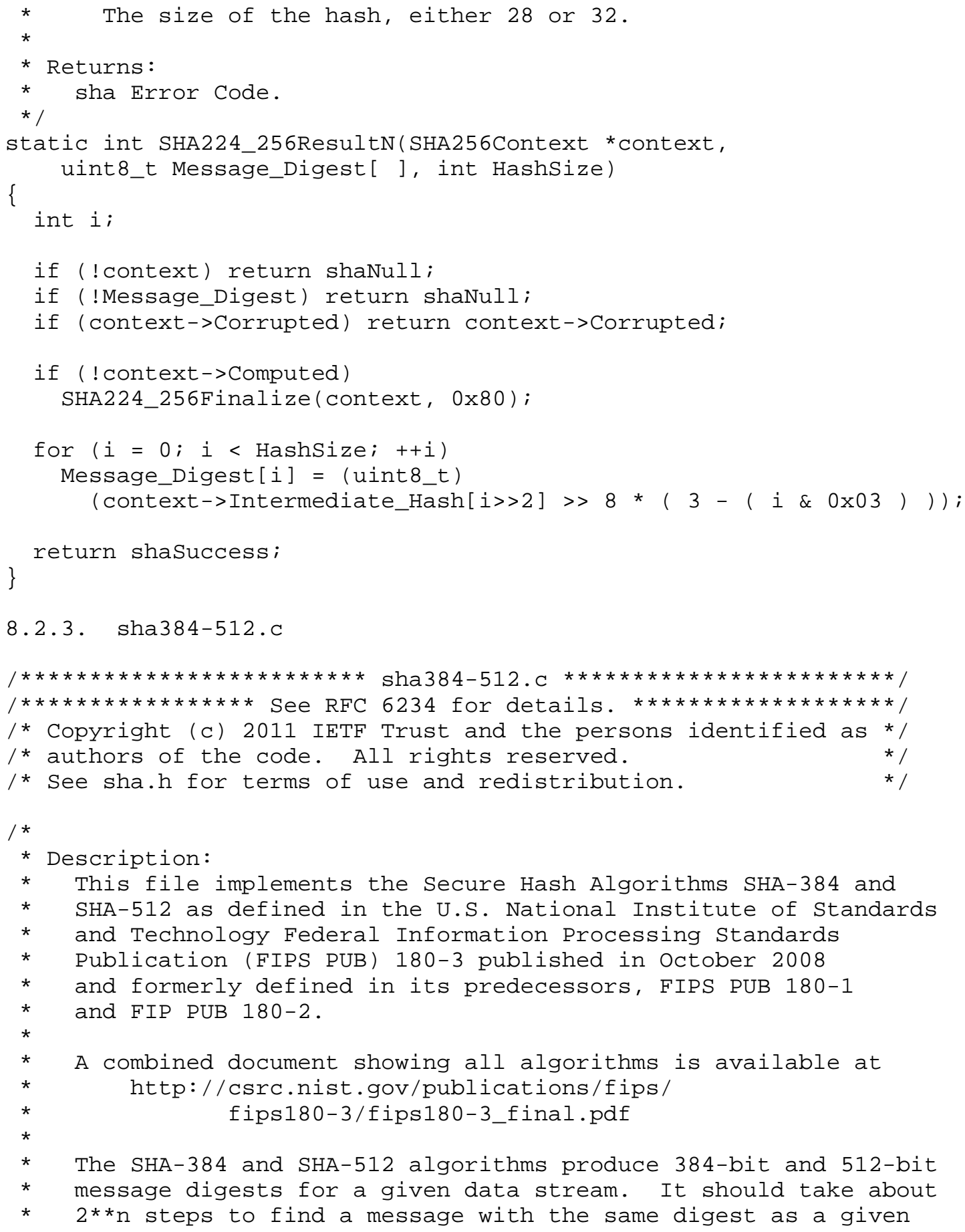




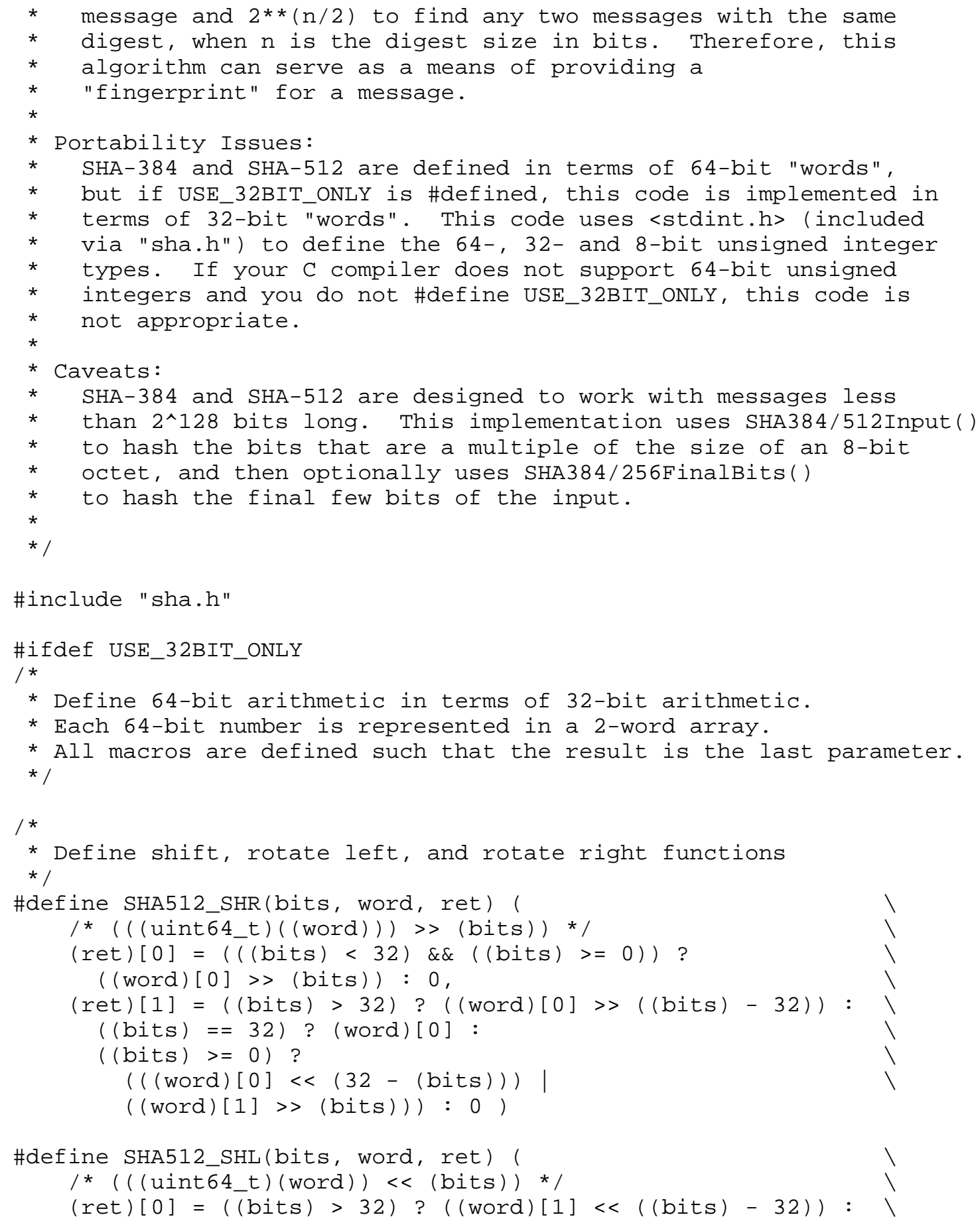




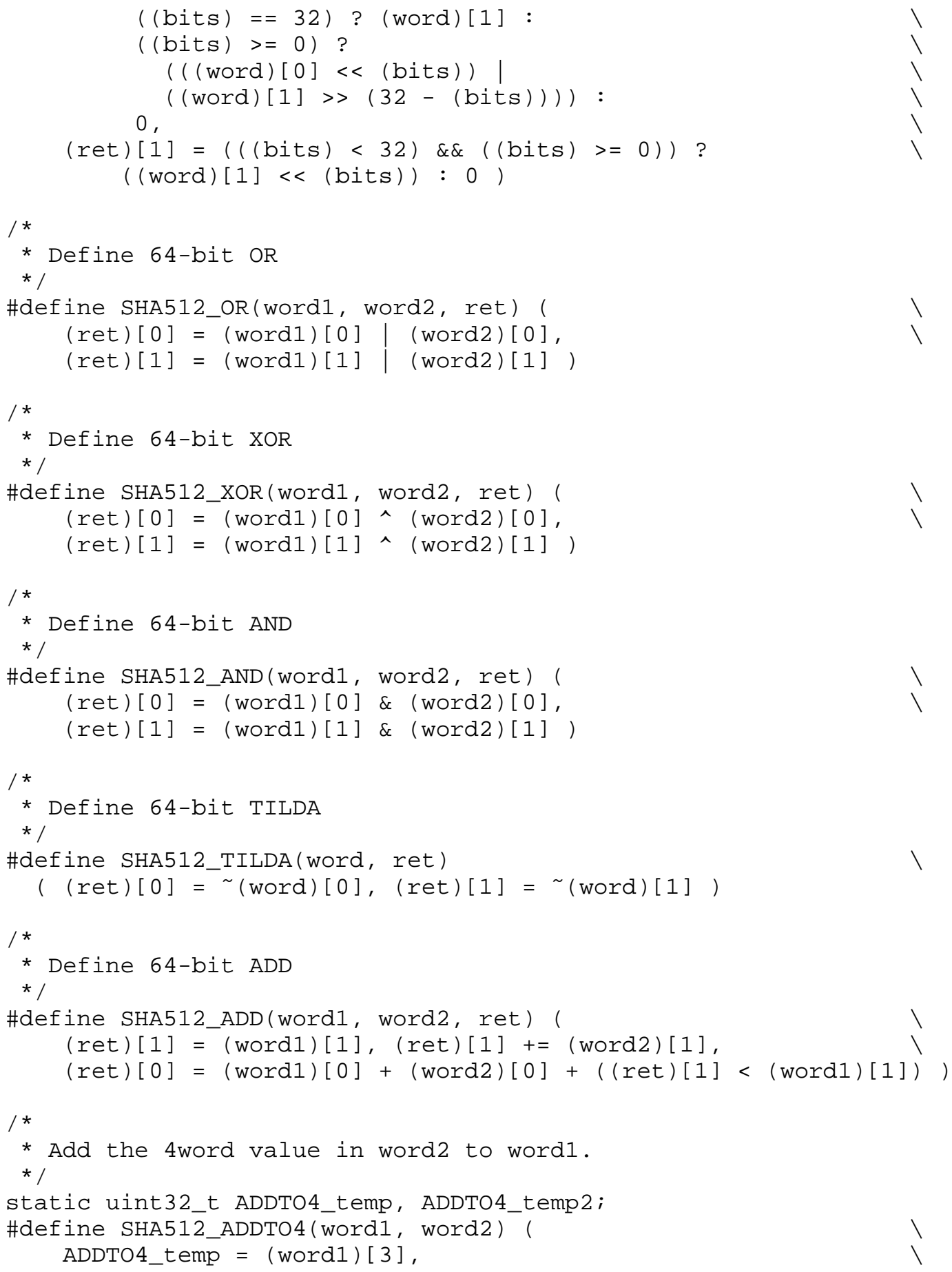




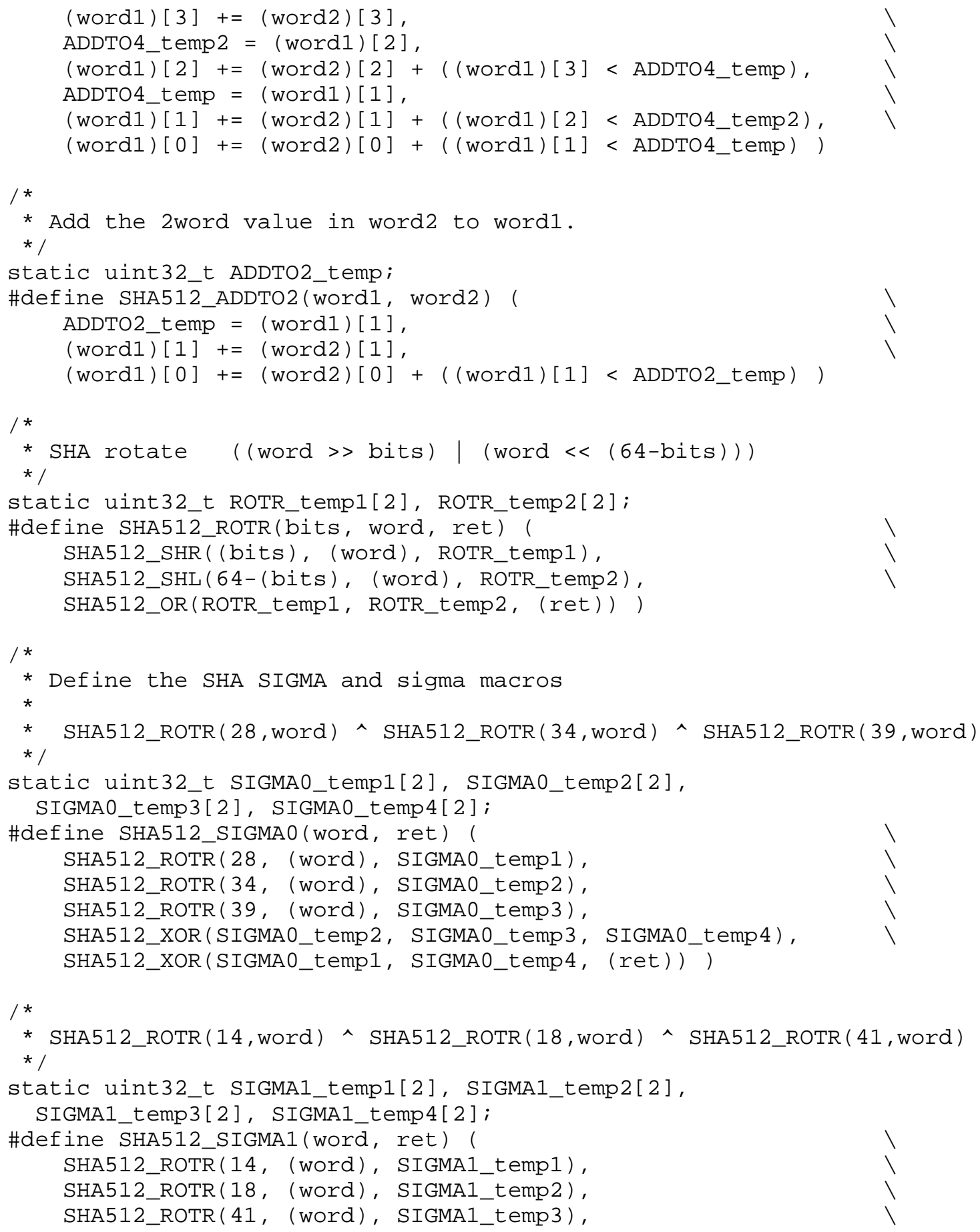




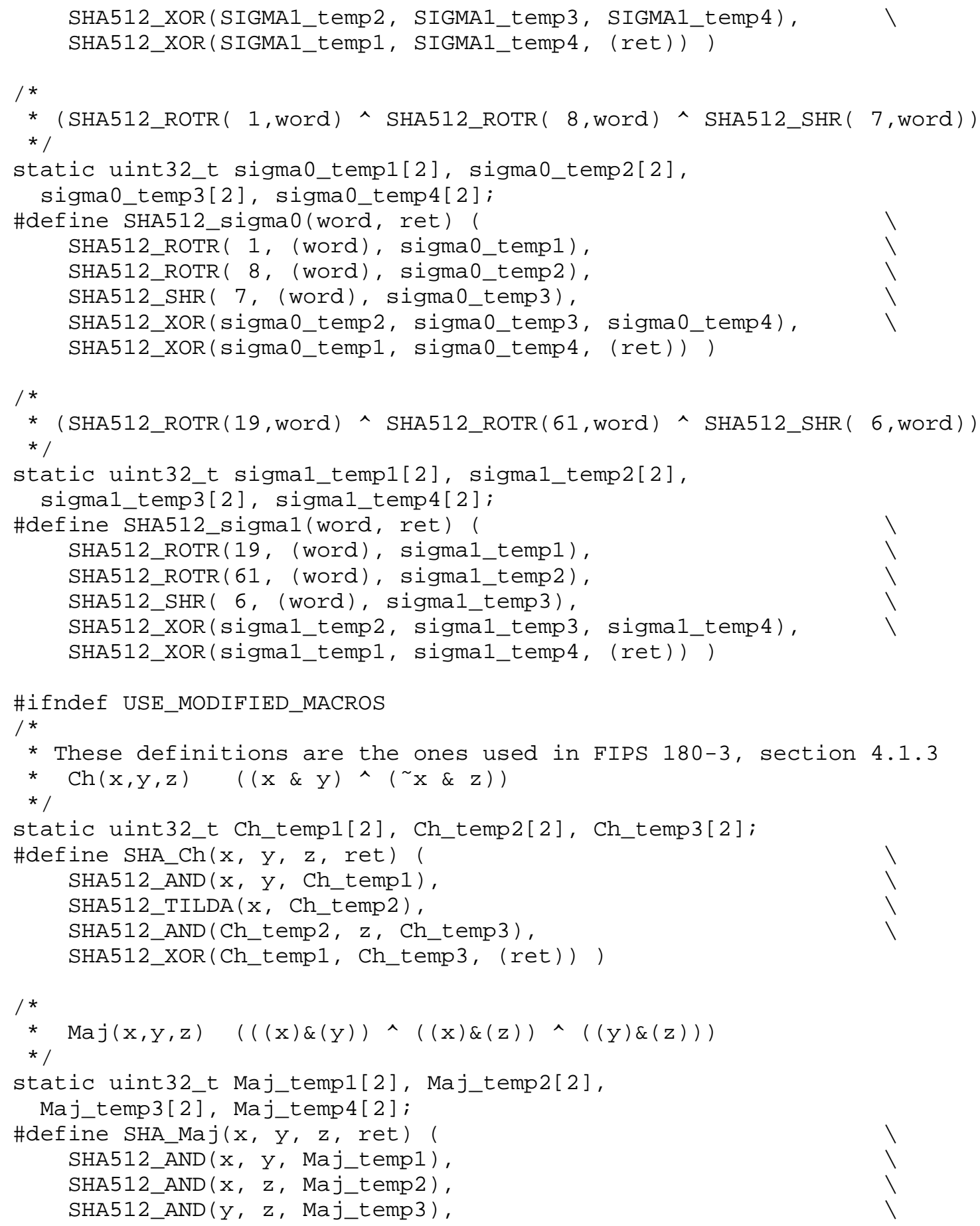




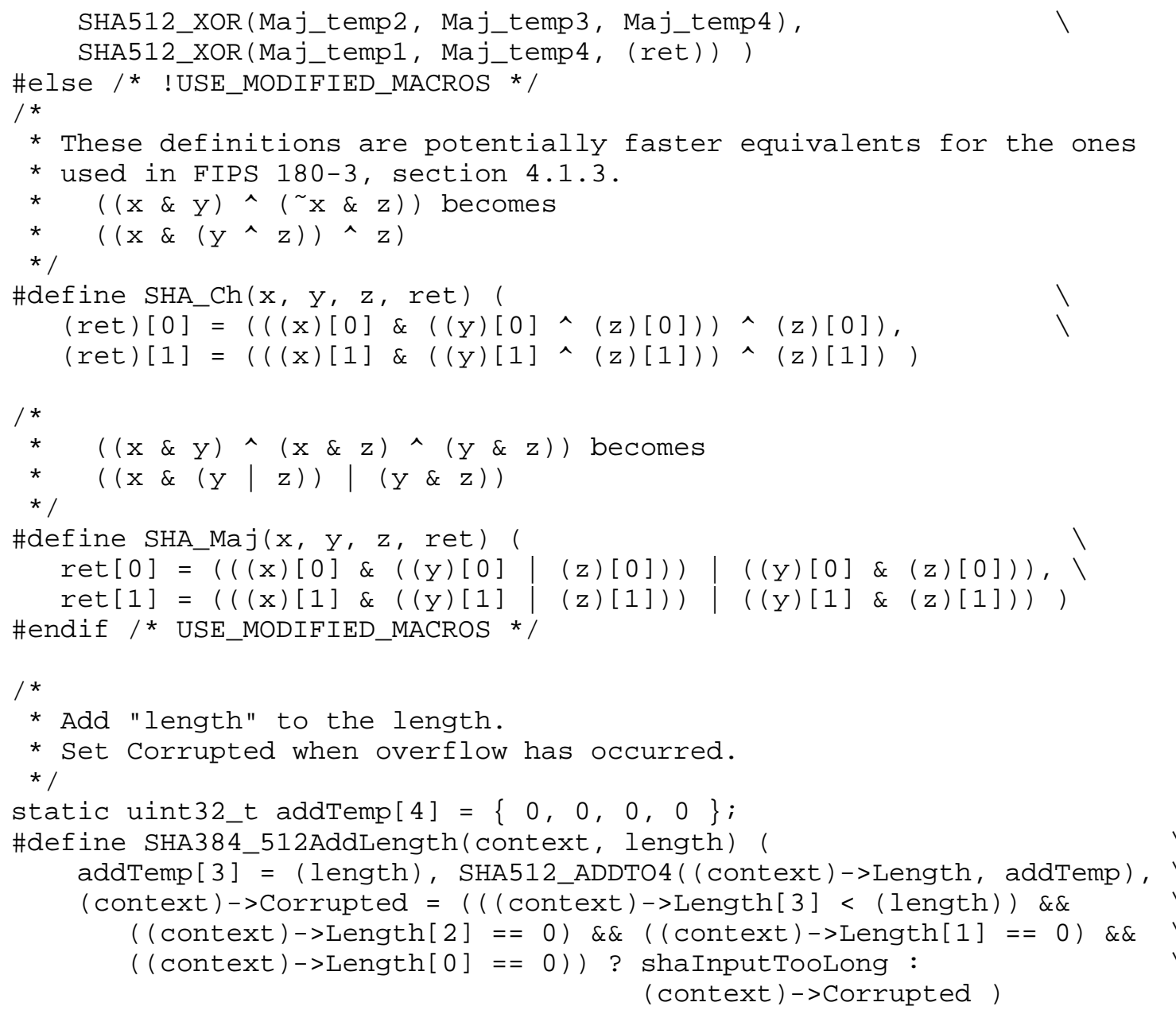




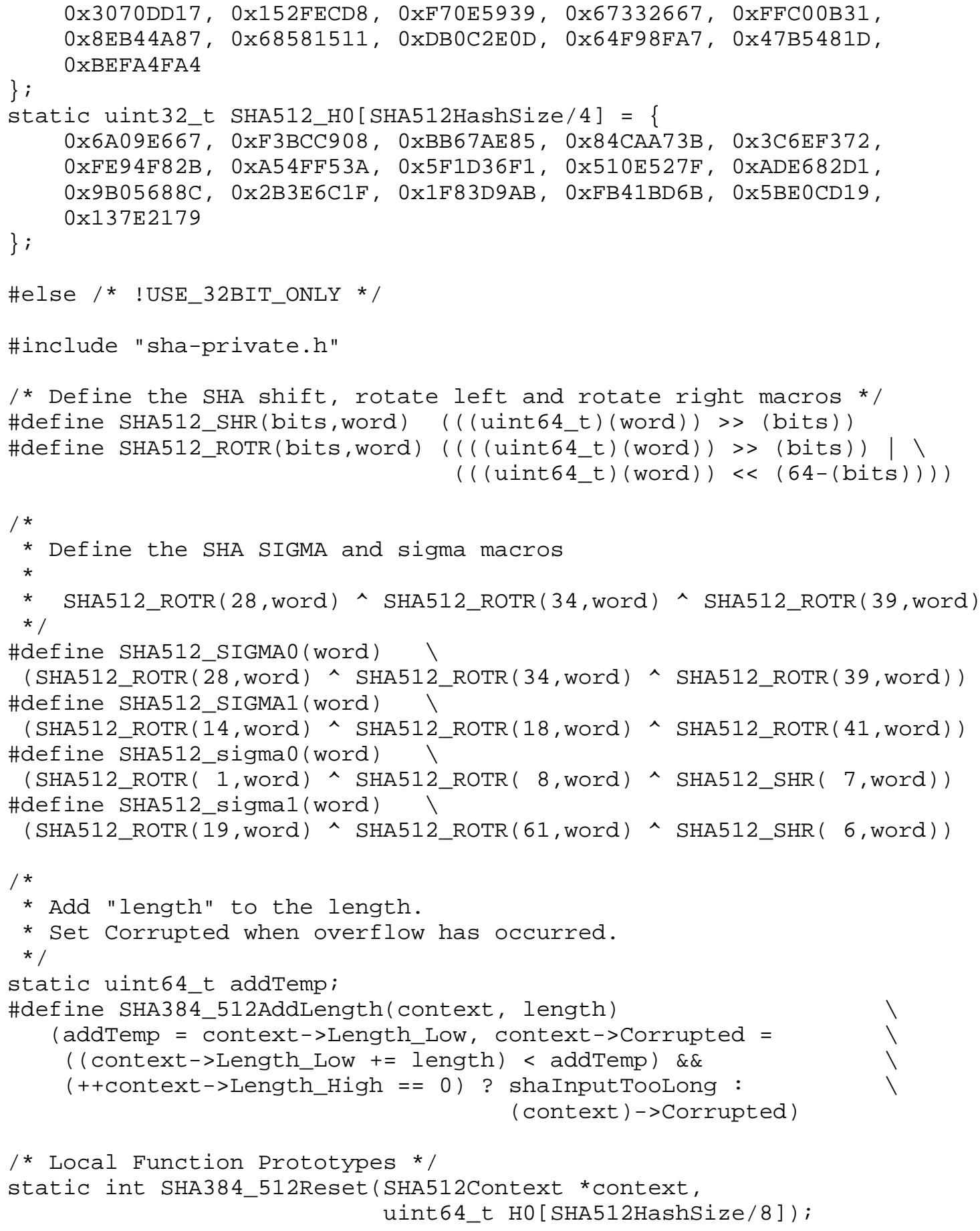




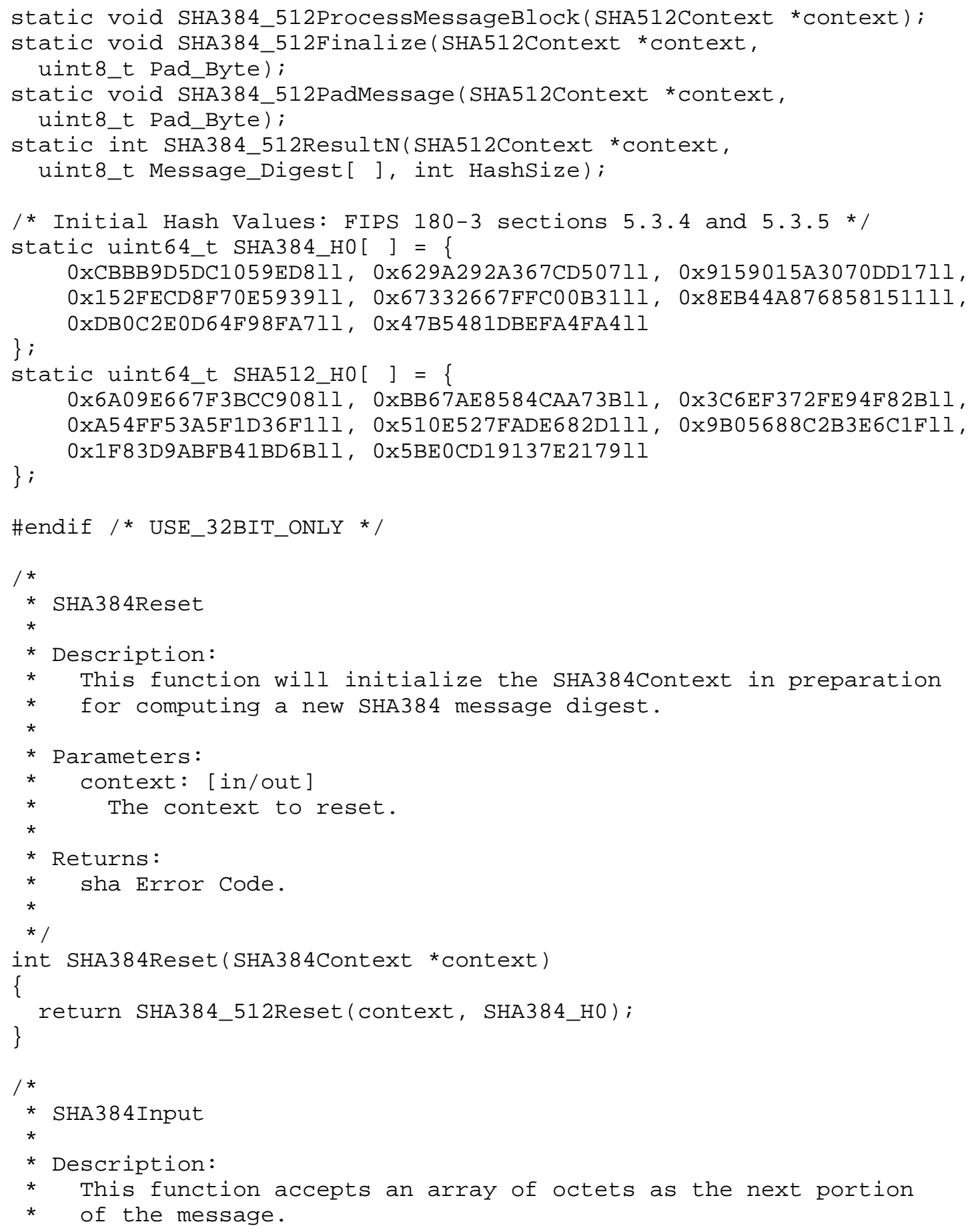




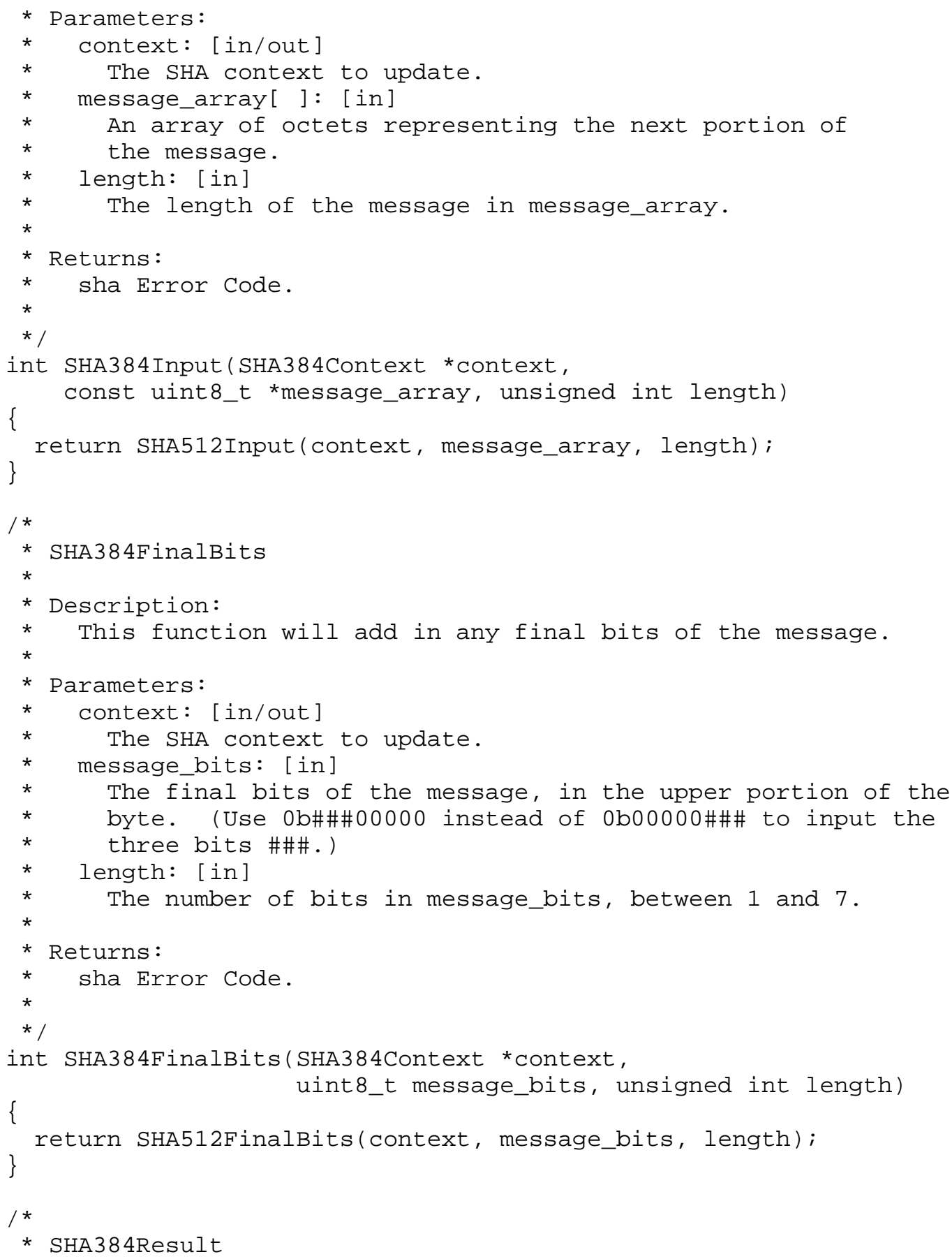




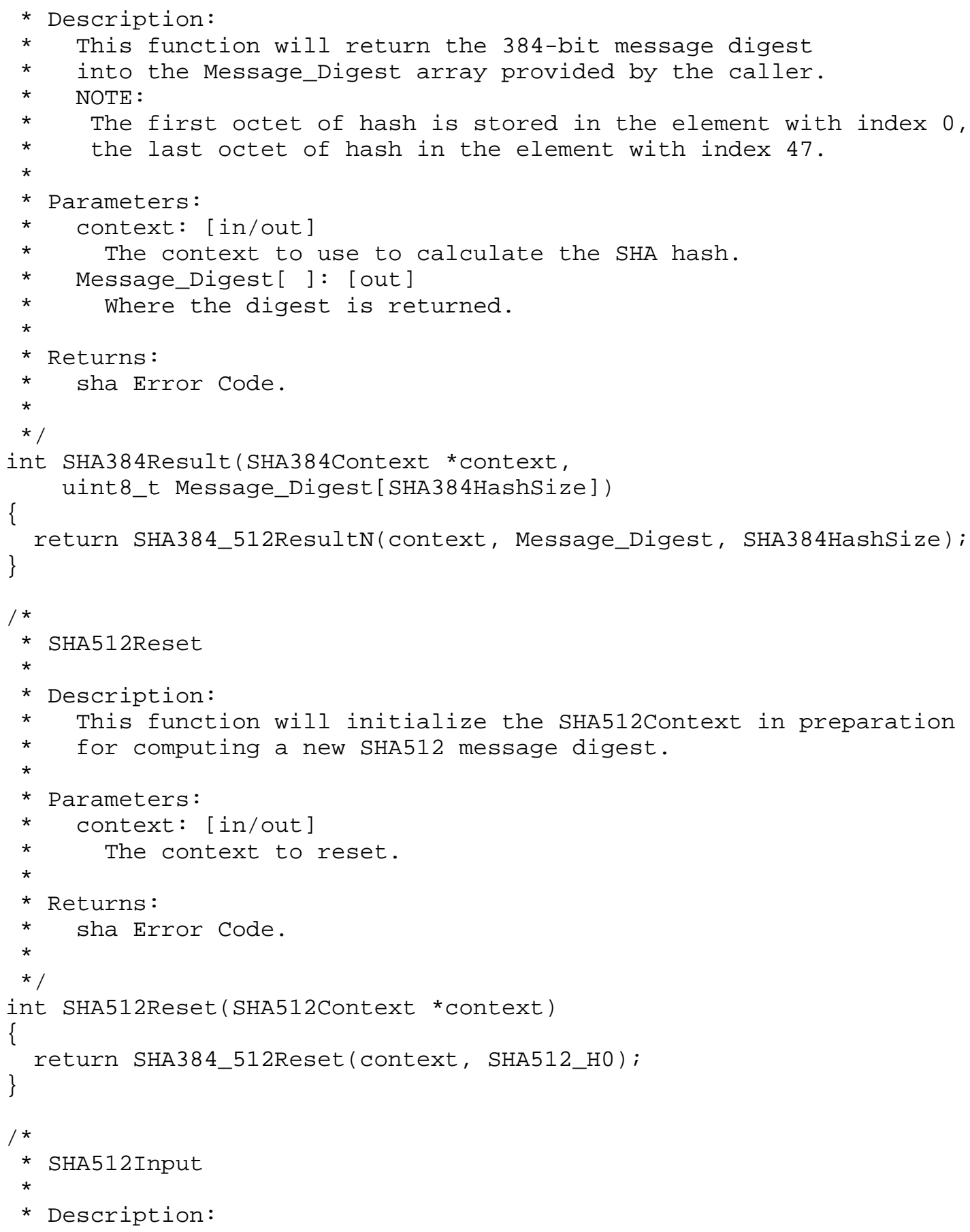




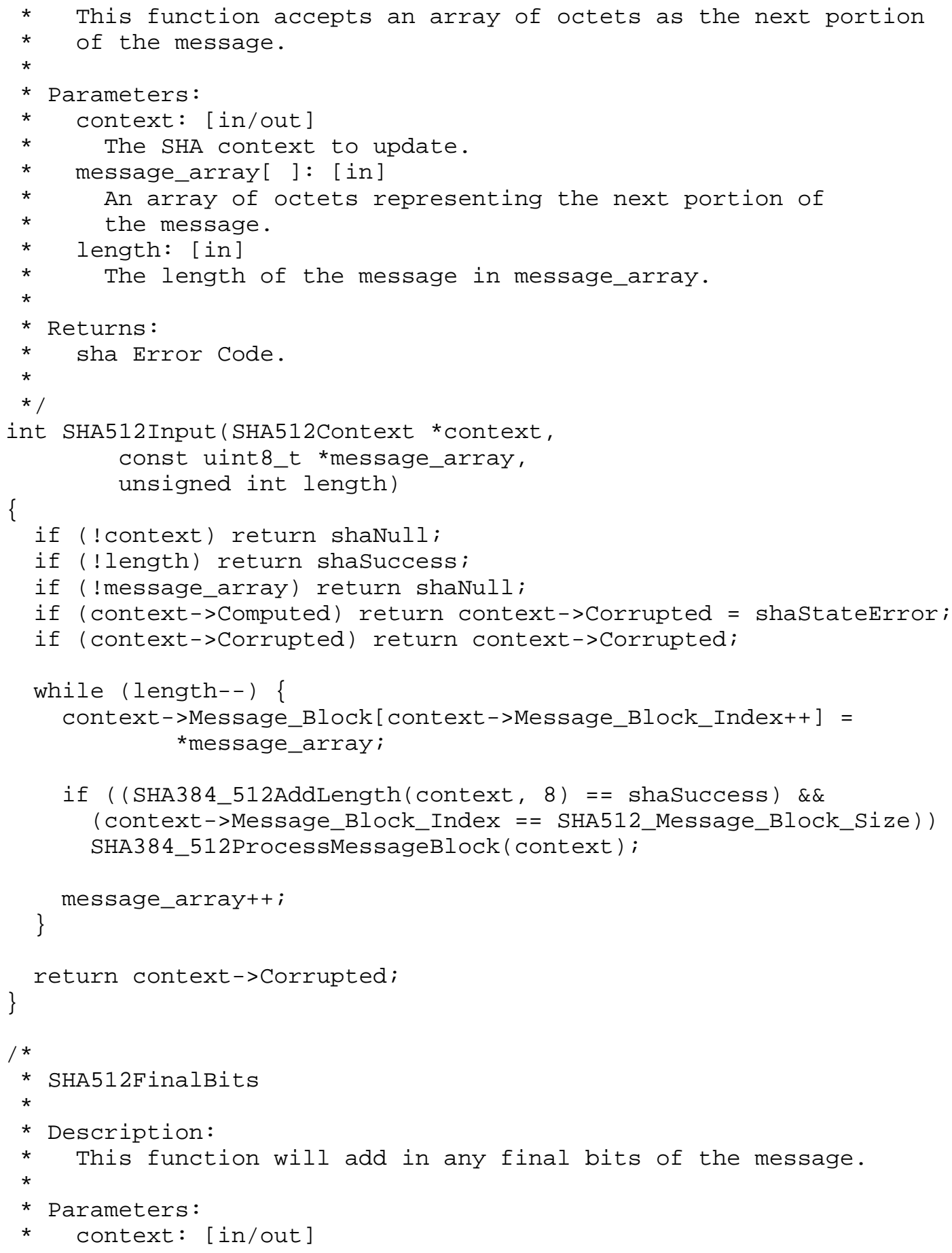




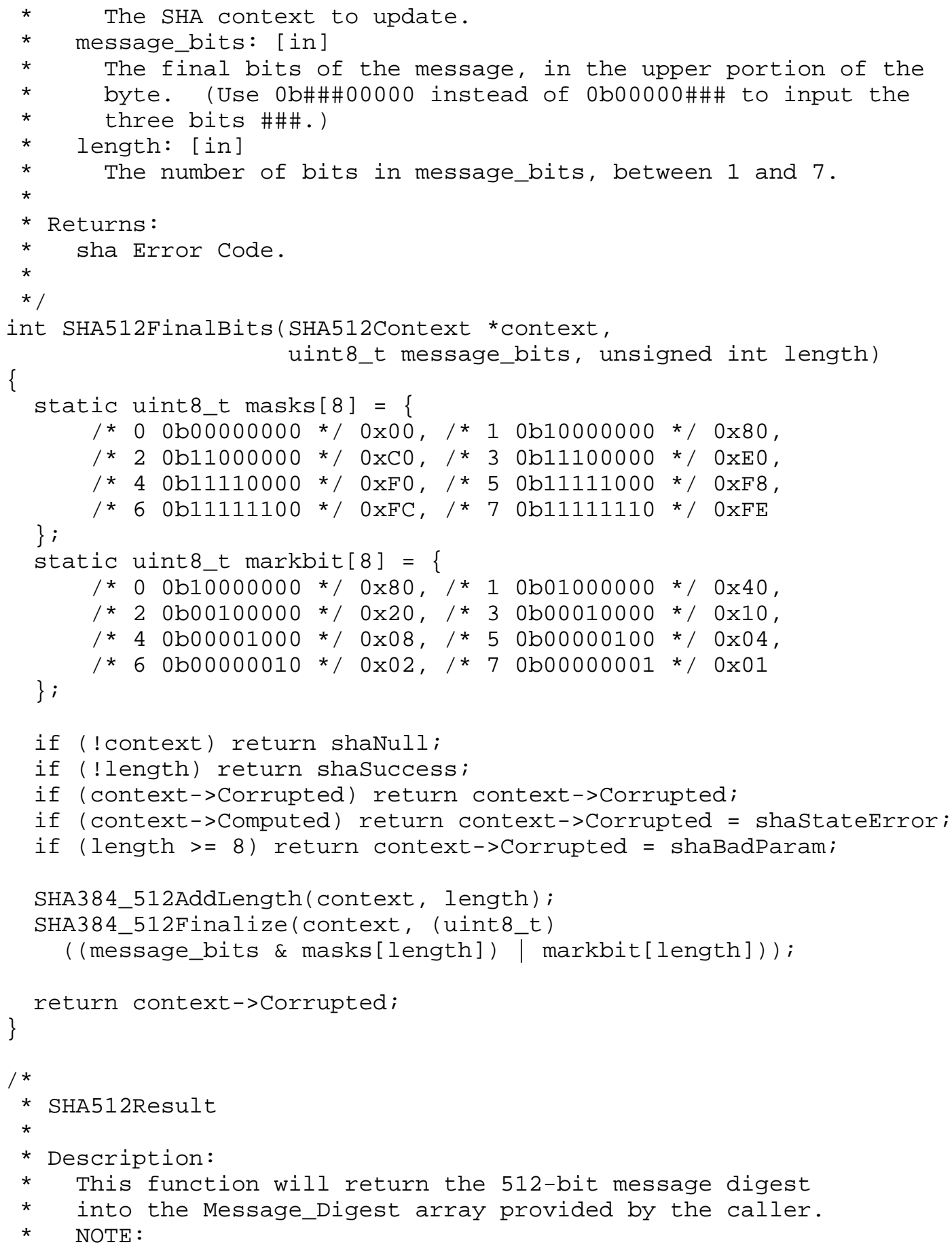




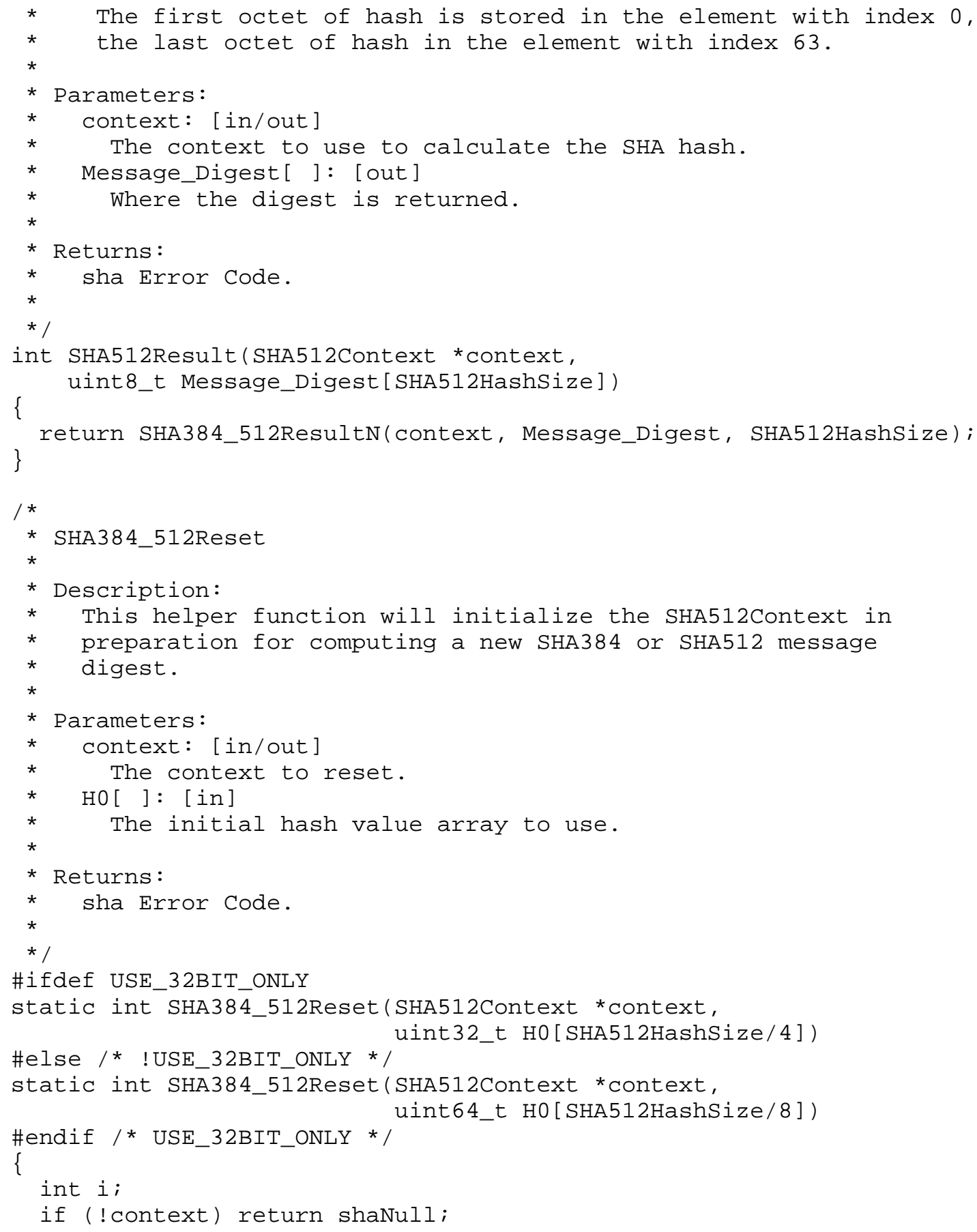




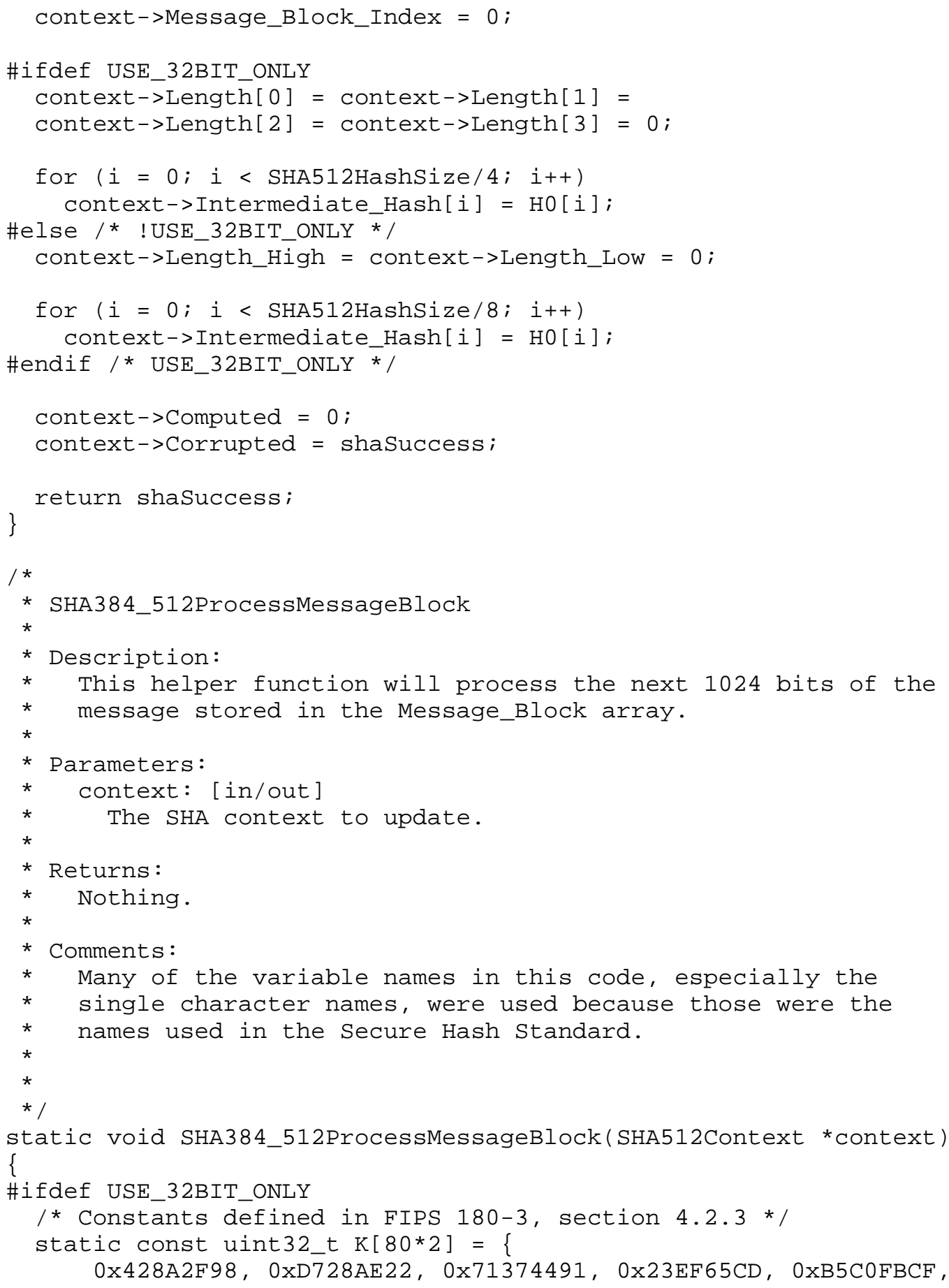




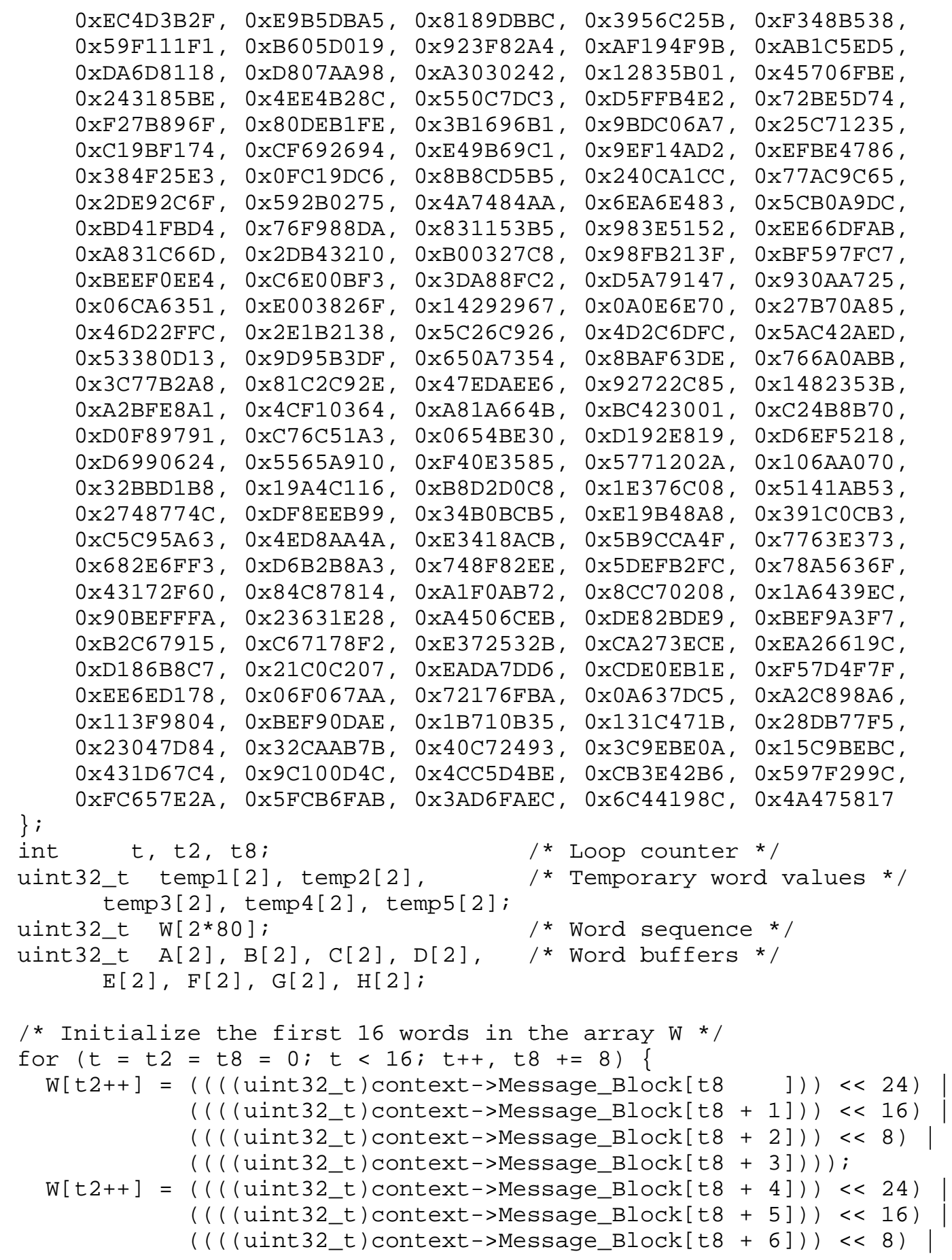


\}

$(((($ uint32_t $)$ context->Message_Block $[$ t8 + 7] $)))$;

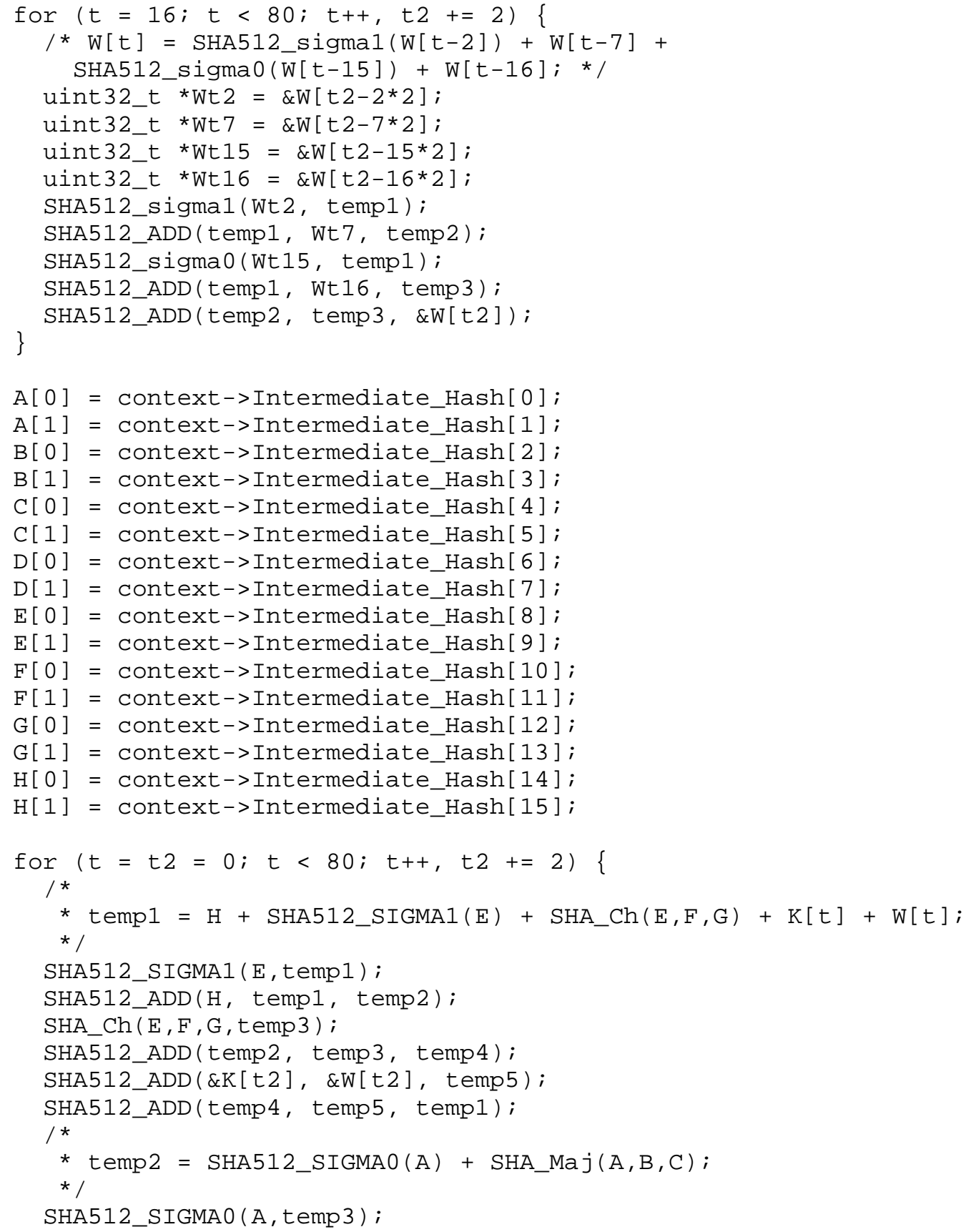


SHA_Maj (A, B, C, temp4);

SHA512_ADD (temp3, temp4, temp2);

$\mathrm{H}[0]=\mathrm{G}[0] ; \mathrm{H}[1]=\mathrm{G}[1]$;

$\mathrm{G}[0]=\mathrm{F}[0] ; \mathrm{G}[1]=\mathrm{F}[1] ;$

$\mathrm{F}[0]=\mathrm{E}[0] ; \mathrm{F}[1]=\mathrm{E}[1] ;$

SHA512_ADD (D, temp1, E);

$\mathrm{D}[0]=\mathrm{C}[0] ; \mathrm{D}[1]=\mathrm{C}[1] ;$

$\mathrm{C}[0]=\mathrm{B}[0] ; \mathrm{C}[1]=\mathrm{B}[1] ;$

$\mathrm{B}[0]=\mathrm{A}[0] ; \mathrm{B}[1]=\mathrm{A}[1] ;$

\}

SHA512_ADD (temp1, temp2, A) ;

SHA512_ADDTO2 (\&context->Intermediate_Hash [0], A); SHA512_ADDTO2 (\&context->Intermediate_Hash [2], B); SHA512_ADDTO2 (\&context->Intermediate_Hash [4], C); SHA512_ADDTO2 (\&context->Intermediate_Hash [6], D); SHA512_ADDTO2 (\&context->Intermediate_Hash [8], E); SHA512_ADDTO2 (\&context->Intermediate_Hash [10], F); SHA512_ADDTO2 (\&context->Intermediate_Hash [12], G); SHA512_ADDTO2 (\&context->Intermediate_Hash [14], H) ;

\#else /* !USE_32BIT_ONLY */

/* Constants defined in FIPS 180-3, section $4.2 .3 * /$

static const uint64_t $\mathrm{K}[80]=\{$

0x428A2F98D728AE2211, 0x7137449123EF65CDIl, 0xB5C0FBCFEC4D3B2Fll, 0xE9B5DBA58189DBBCl1, 0x3956C25BF348B53811, 0x59F111F1B605D01911, 0x923F82A4AF194F9B11, 0xAB1C5ED5DA6D811811, 0xD807AA98A303024211, 0x12835B0145706FBEll, 0x243185BE4EE4B28Cll, 0x550C7DC3D5FFB4E211, 0x72BE5D74F27B896Fll, 0x80DEB1FE3B1696B111, 0x9BDC06A725C7123511, 0xC19BF174CF69269411, 0xE49B69C19EF14AD211, 0xEFBE4786384F25E311, 0x0FC19DC68B8CD5B511, 0x240CA1CC77AC9C6511, 0x2DE92C6F592B027511, 0x4A7484AA6EA6E48311, 0x5CB0A9DCBD41FBD411, 0x76F988DA831153B511, 0x983E5152EE66DFAB11, 0xA831C66D2DB4321011, 0xB00327C898FB213F11, 0xBF597FC7BEEF0EE411, 0xC6E00BF33DA88FC211, 0xD5A79147930AA72511, 0x06CA6351E003826Fl1, 0x142929670A0E6E7011, 0x27B70A8546D22FFCl1, 0x2E1B21385C26C92611, 0x4D2C6DFC5AC42AED11, 0x53380D139D95B3DF11, 0x650A73548BAF63DEll, 0x766A0ABB3C77B2A811, 0x81C2C92E47EDAEE611, 0x92722C851482353B11, 0xA2BFE8A14CF1036411, 0xA81A664BBC42300111, 0xC24B8B70D0F8979111, 0xC76C51A30654BE3011, 0xD192E819D6EF521811, 0xD69906245565A91011, OxF40E35855771202Al1, 0x106AA07032BBD1B811, 0x19A4C116B8D2D0C811, 0x1E376C085141AB5311, 0x2748774CDF8EEB9911, 0x34B0BCB5E19B48A811, 0x391C0CB3C5C95A6311, 0x4ED8AA4AE3418ACB11, 0x5B9CCA4F7763E37311, 0x682E6FF3D6B2B8A311, 0x748F82EE5DEFB2FCl1, 0x78A5636F43172F6011, 0x84C87814A1F0AB7211, 0x8CC702081A6439ECl1, 0x90BEFFFA23631E2811, 0xA4506CEBDE82BDE911, 0xBEF9A3F7B2C6791511, 0xC67178F2E372532Bll, 0xCA273ECEEA26619Cl1, 0xD186B8C721C0C20711, 0xEADA7DD6CDE0EB1E11, 0xF57D4F7FEE6ED17811, 0x06F067AA72176FBA11, 0x0A637DC5A2C898A611, 0x113F9804BEF90DAE11, 0x1B710B35131C471B11, 


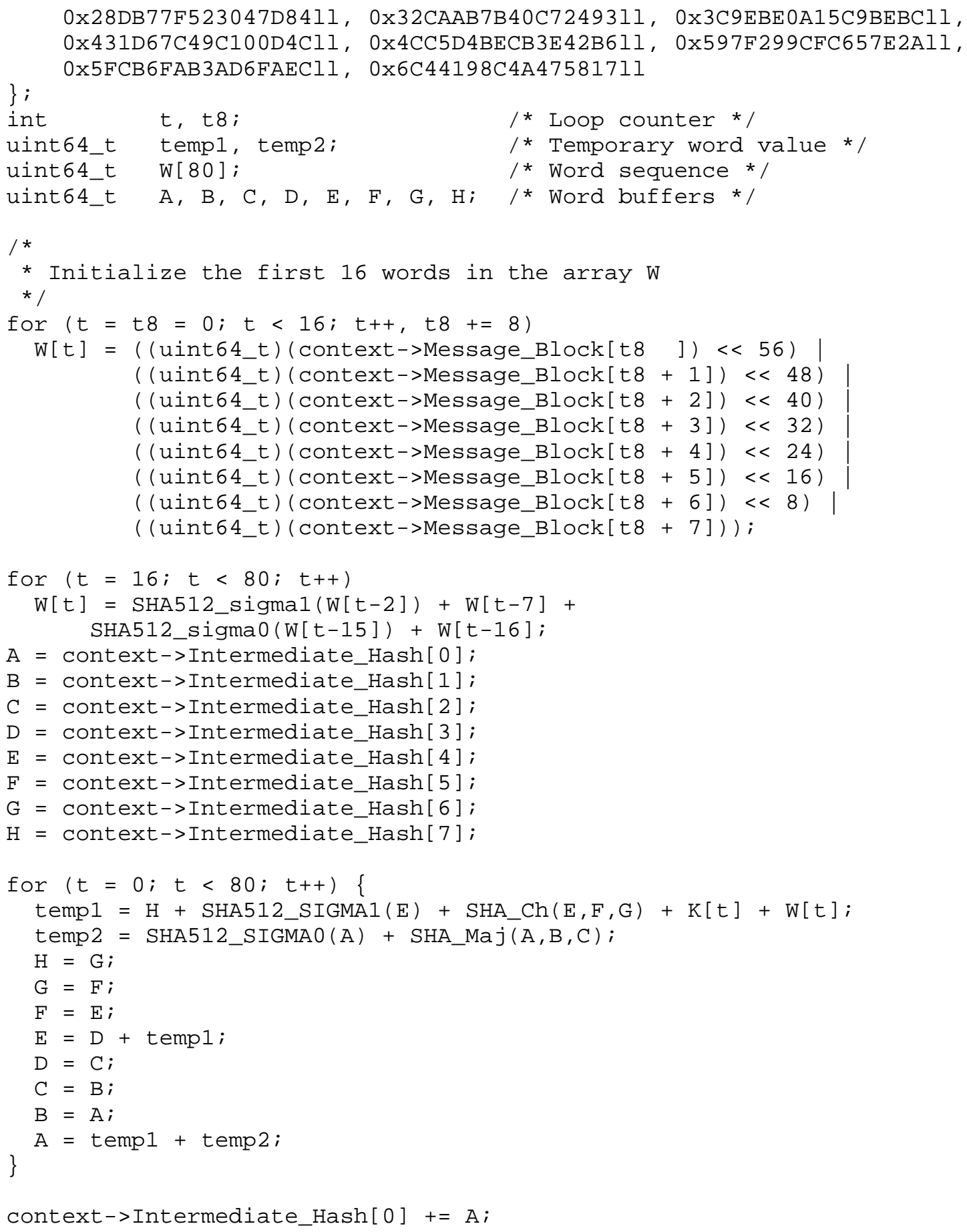




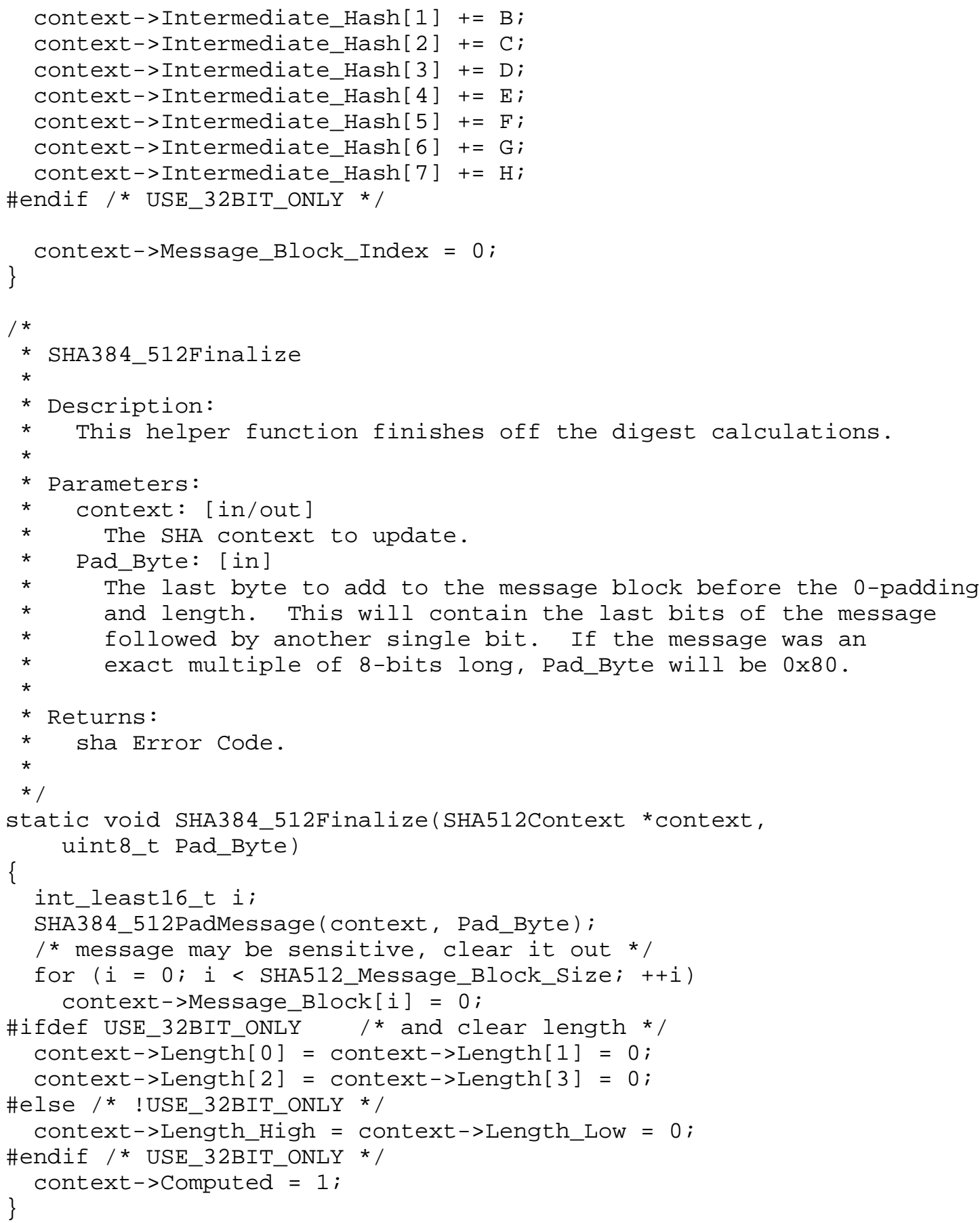




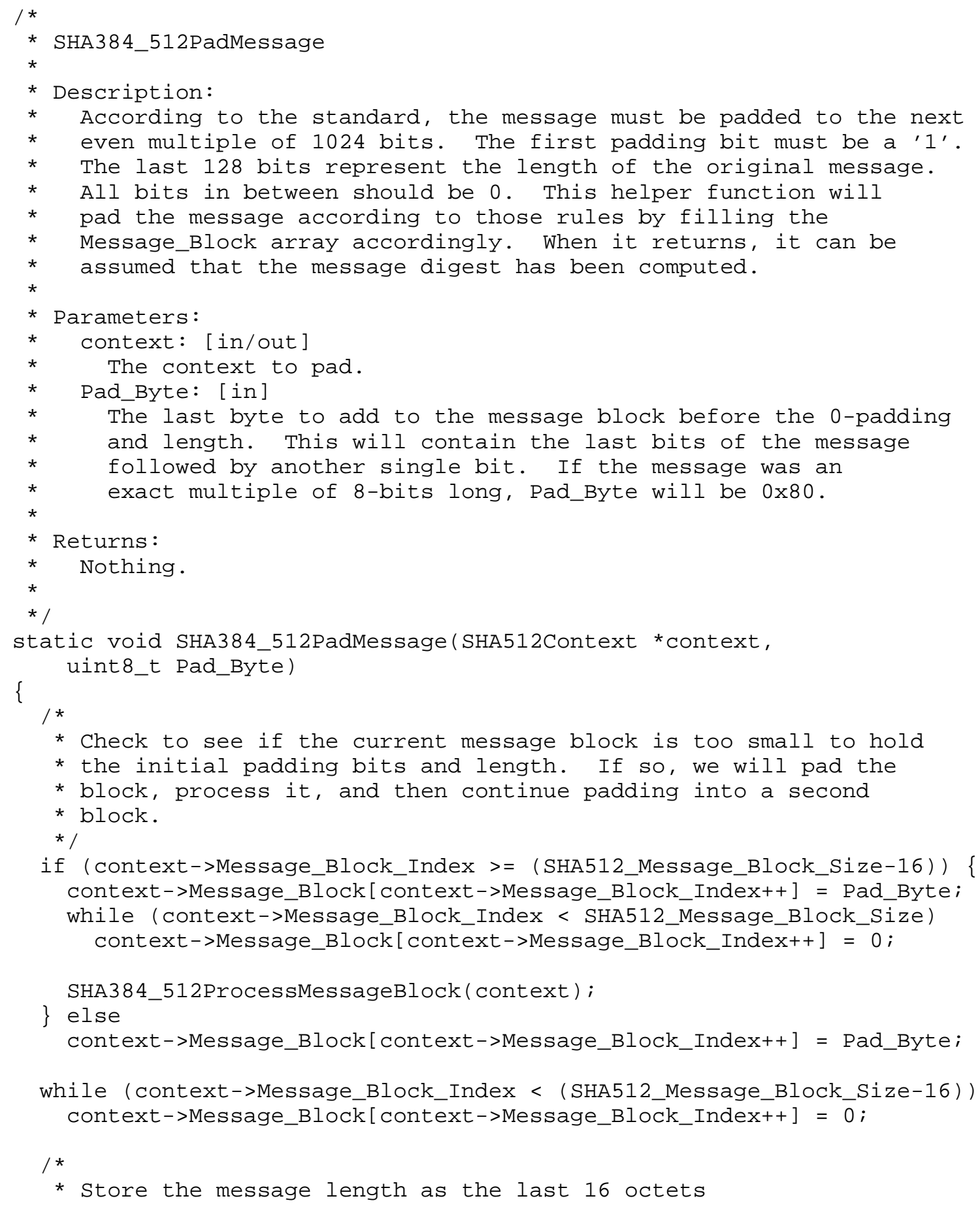




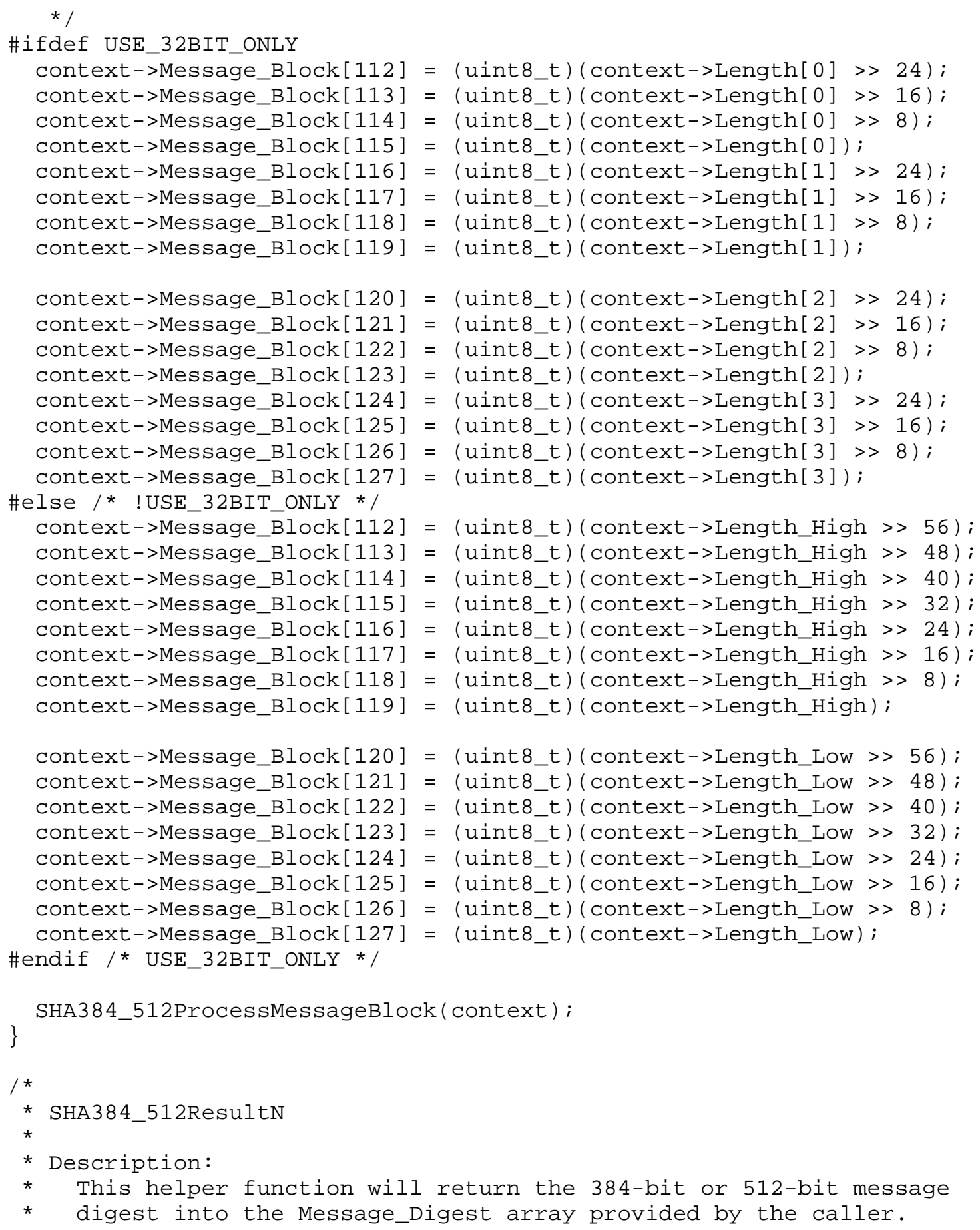




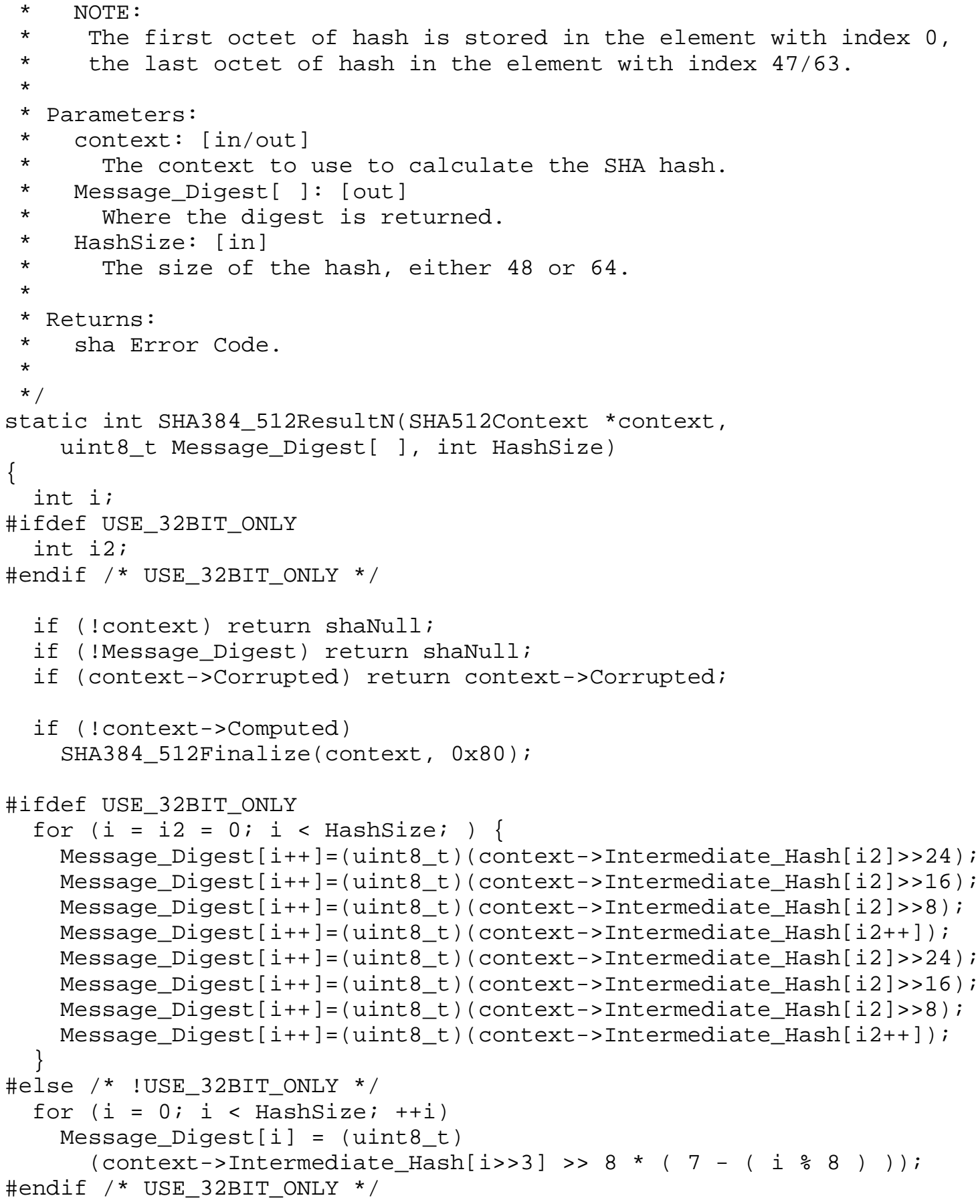




$$
\text { \} }
$$

return shasuccess;

8.2.4. usha.c

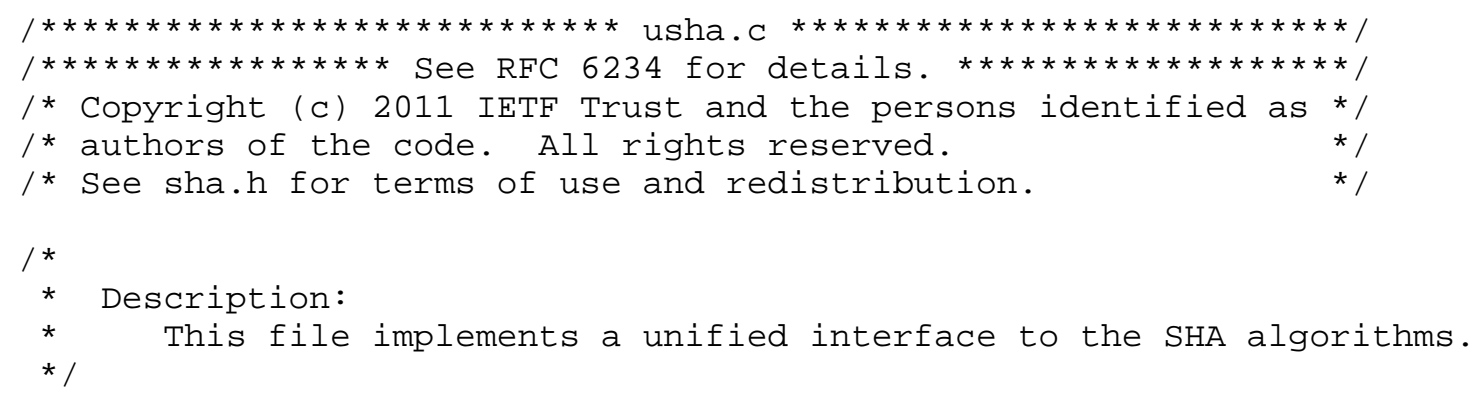




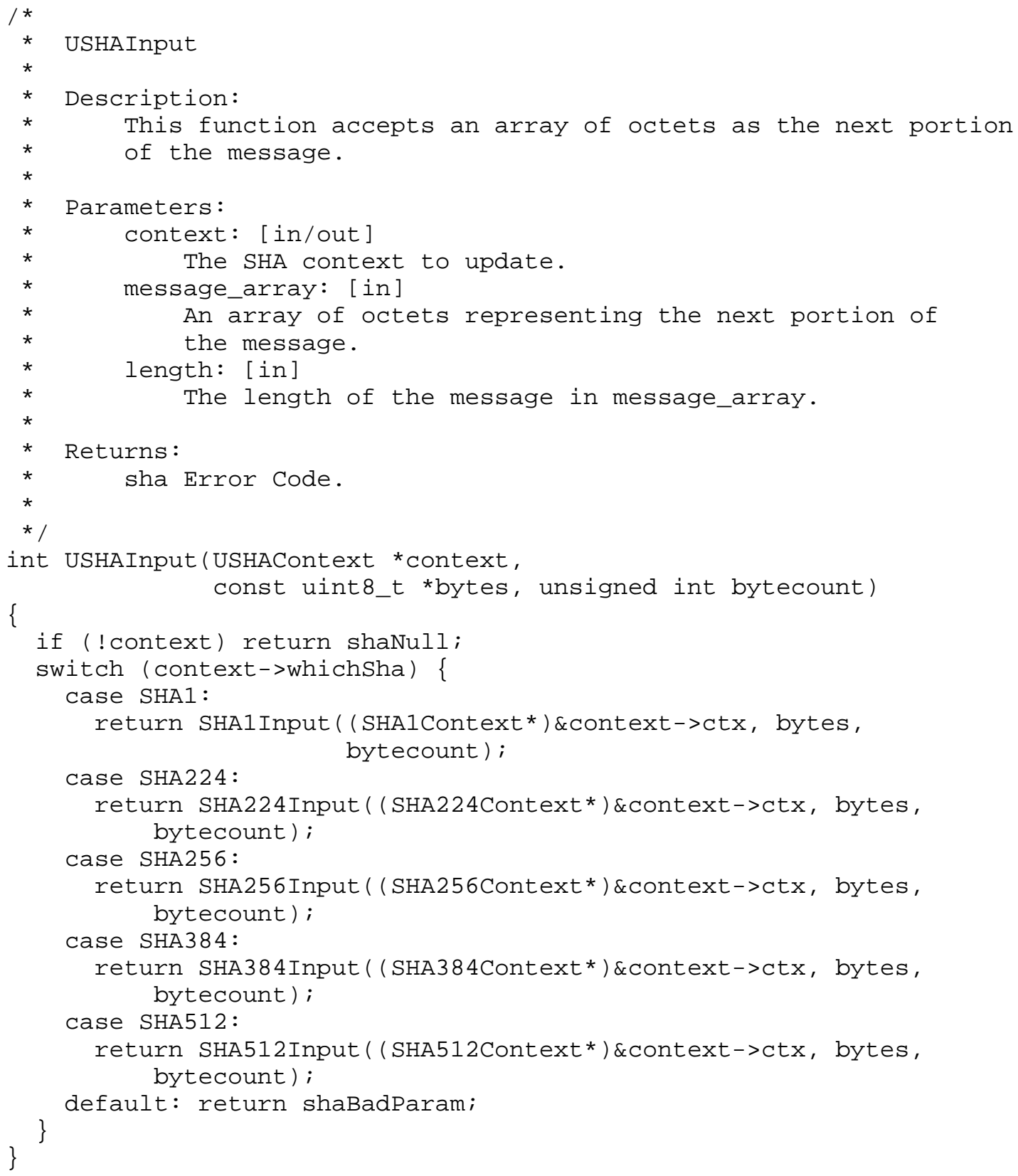




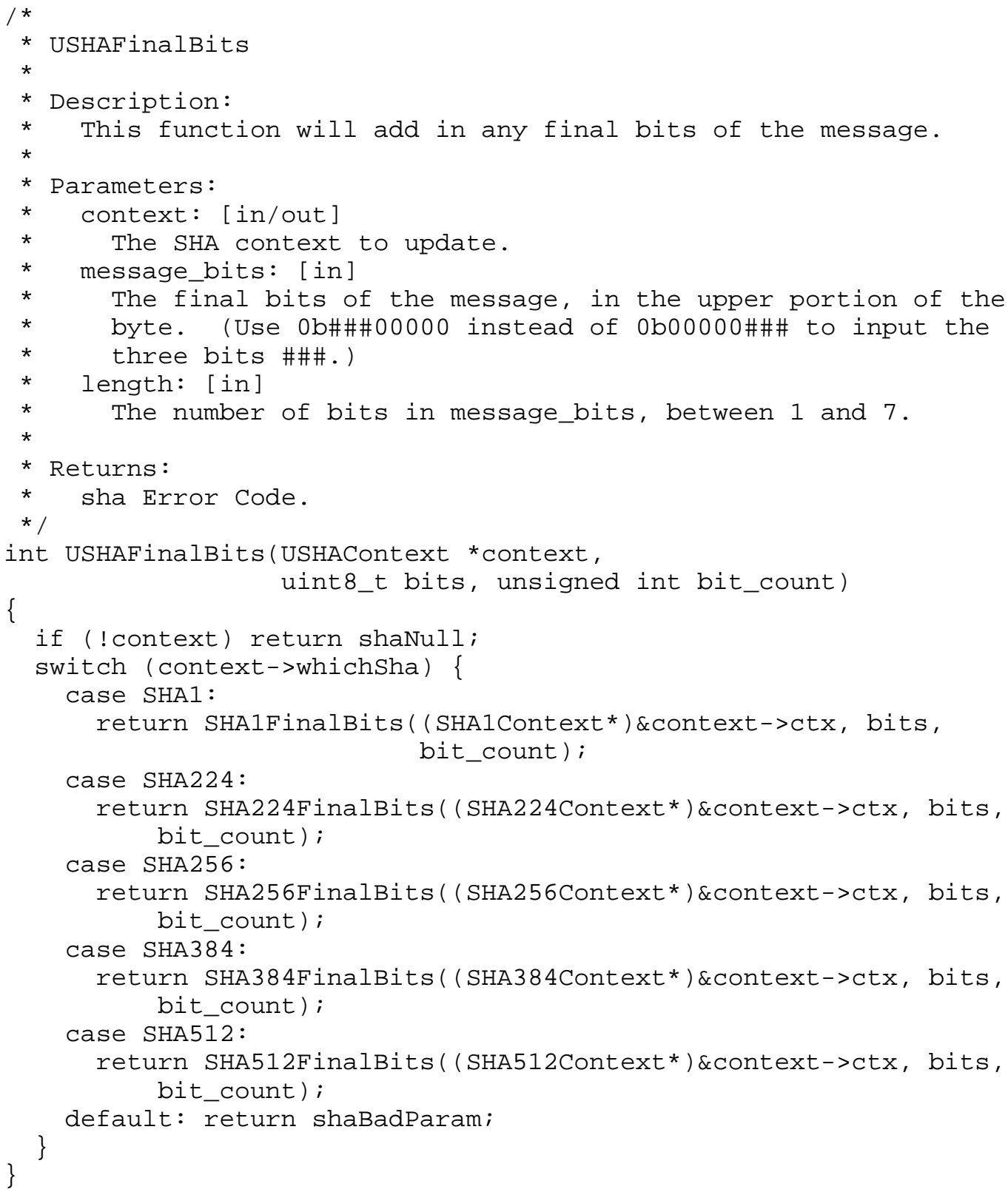




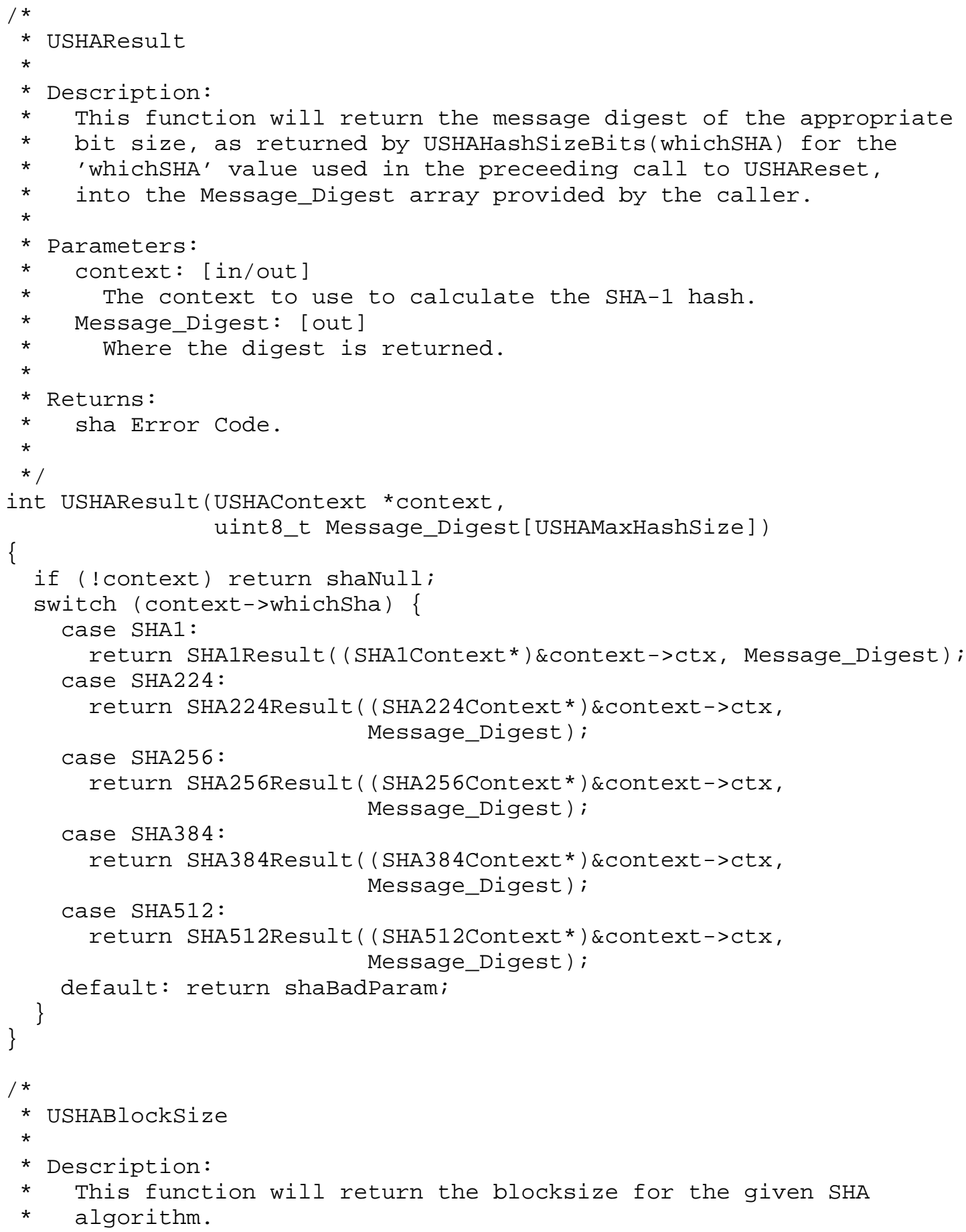




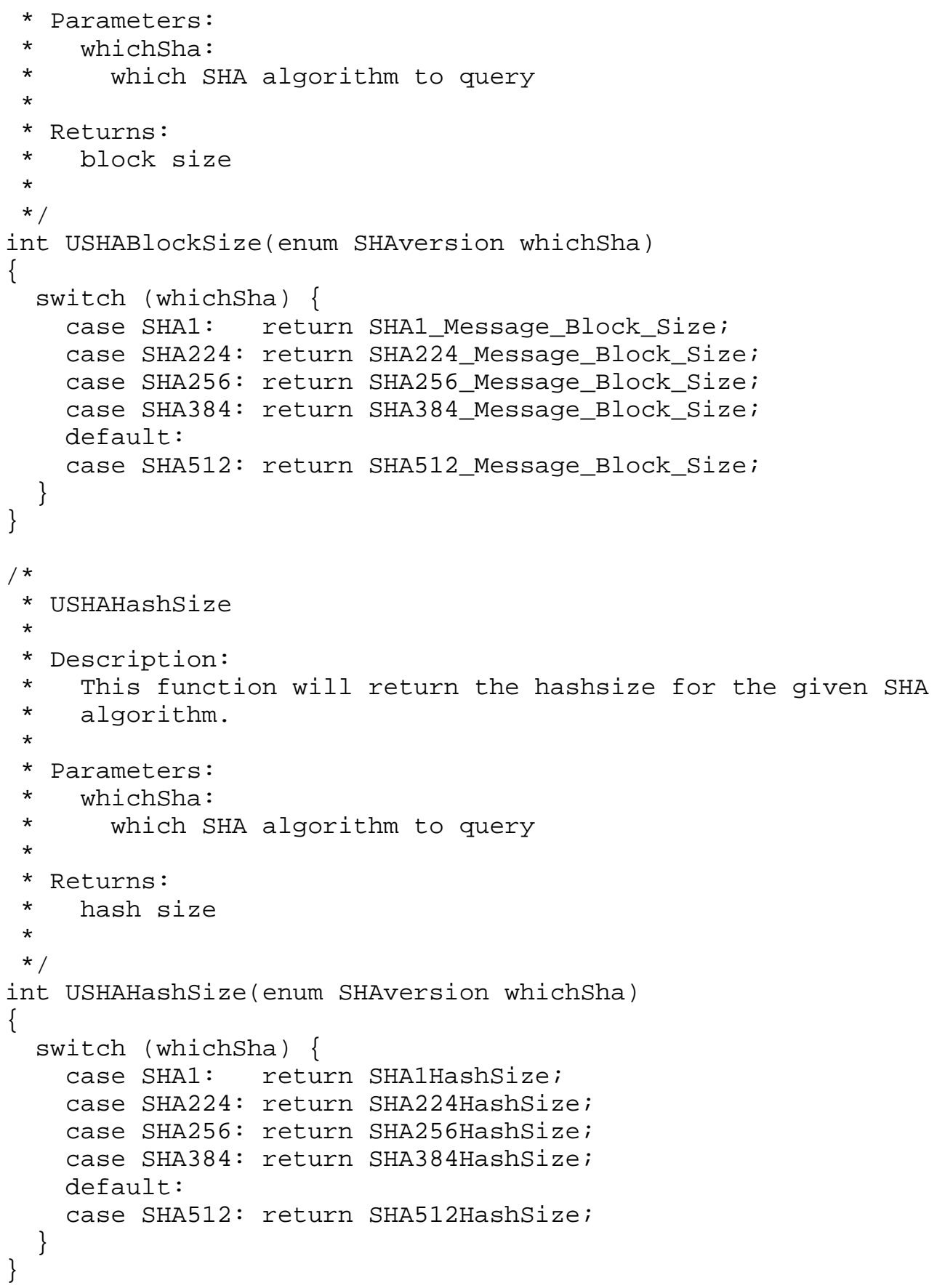




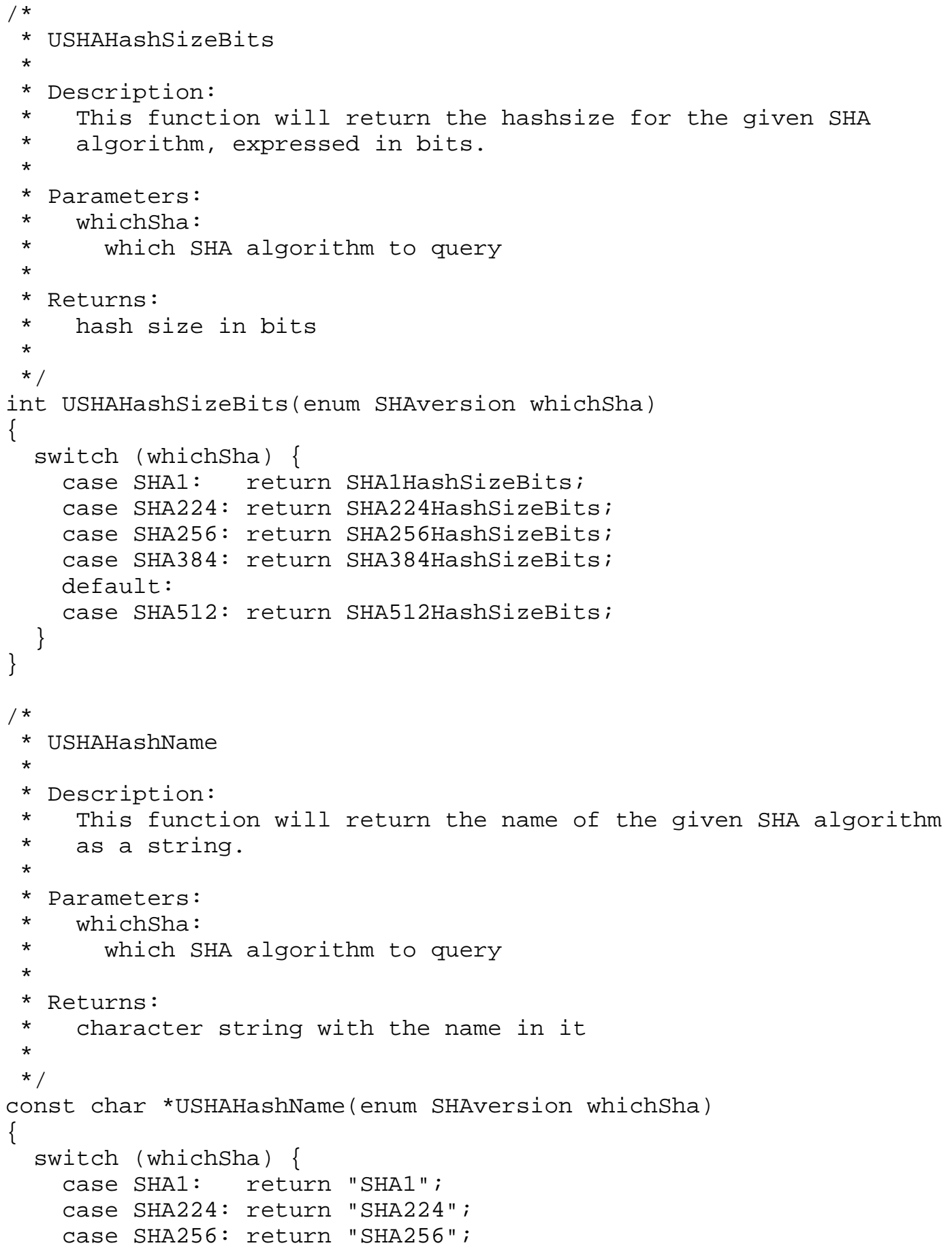




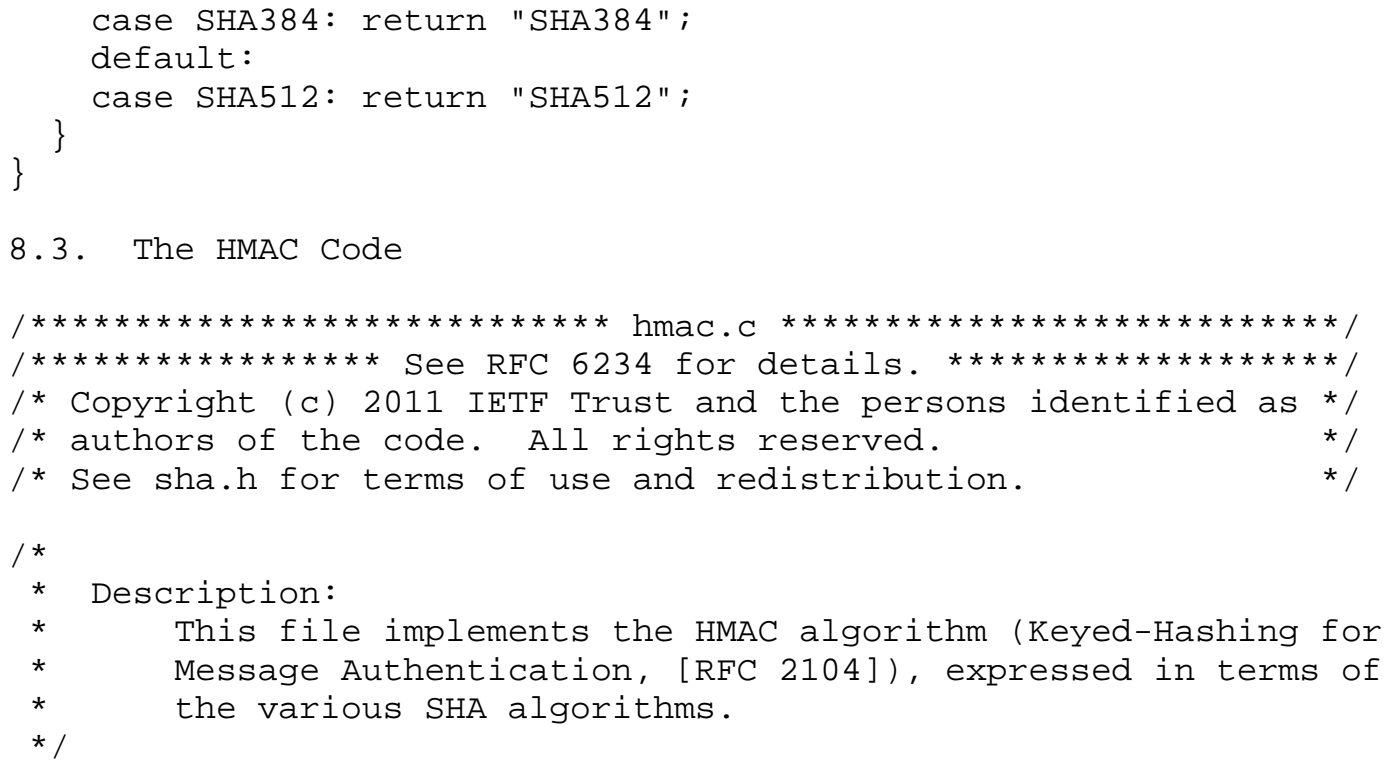




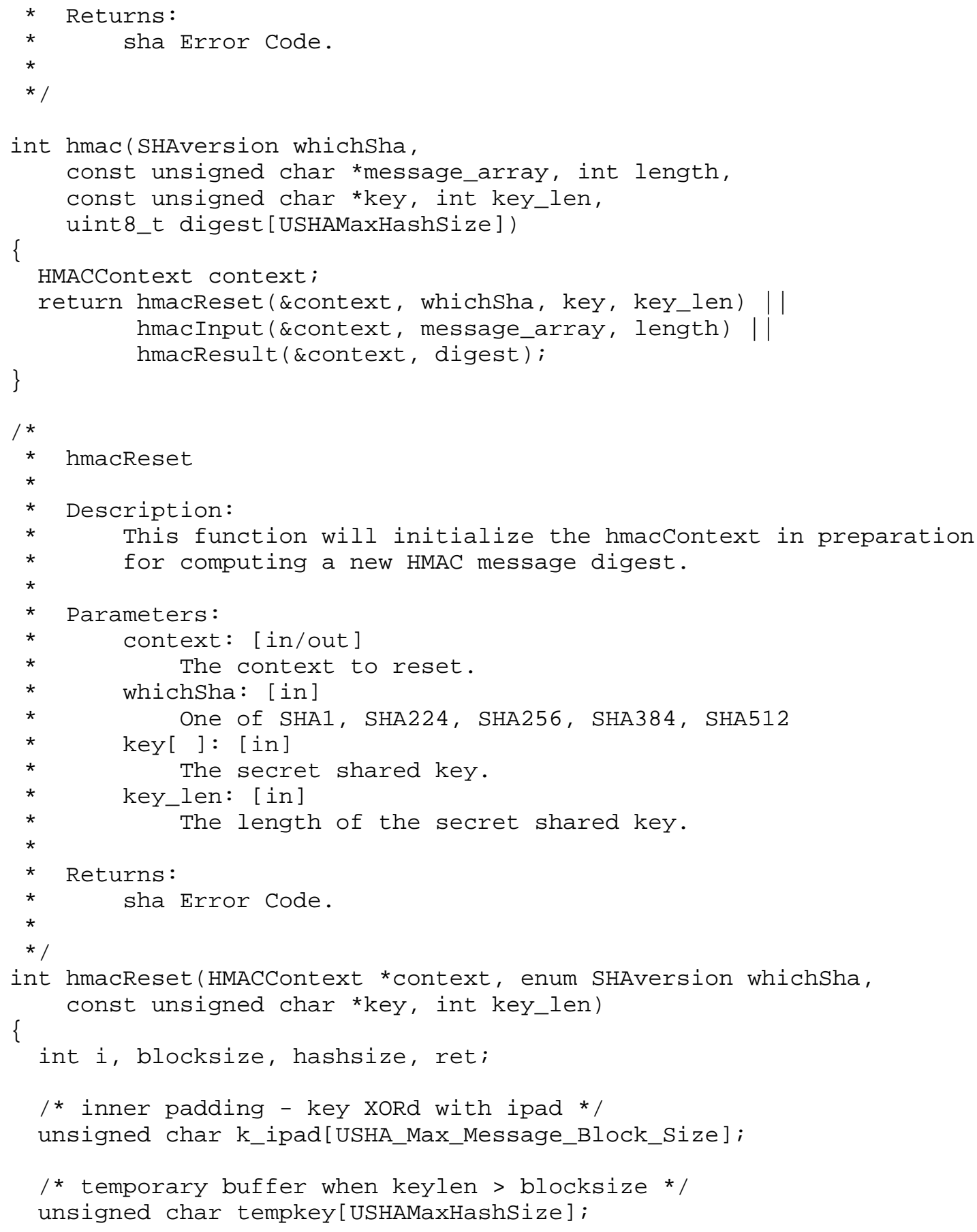




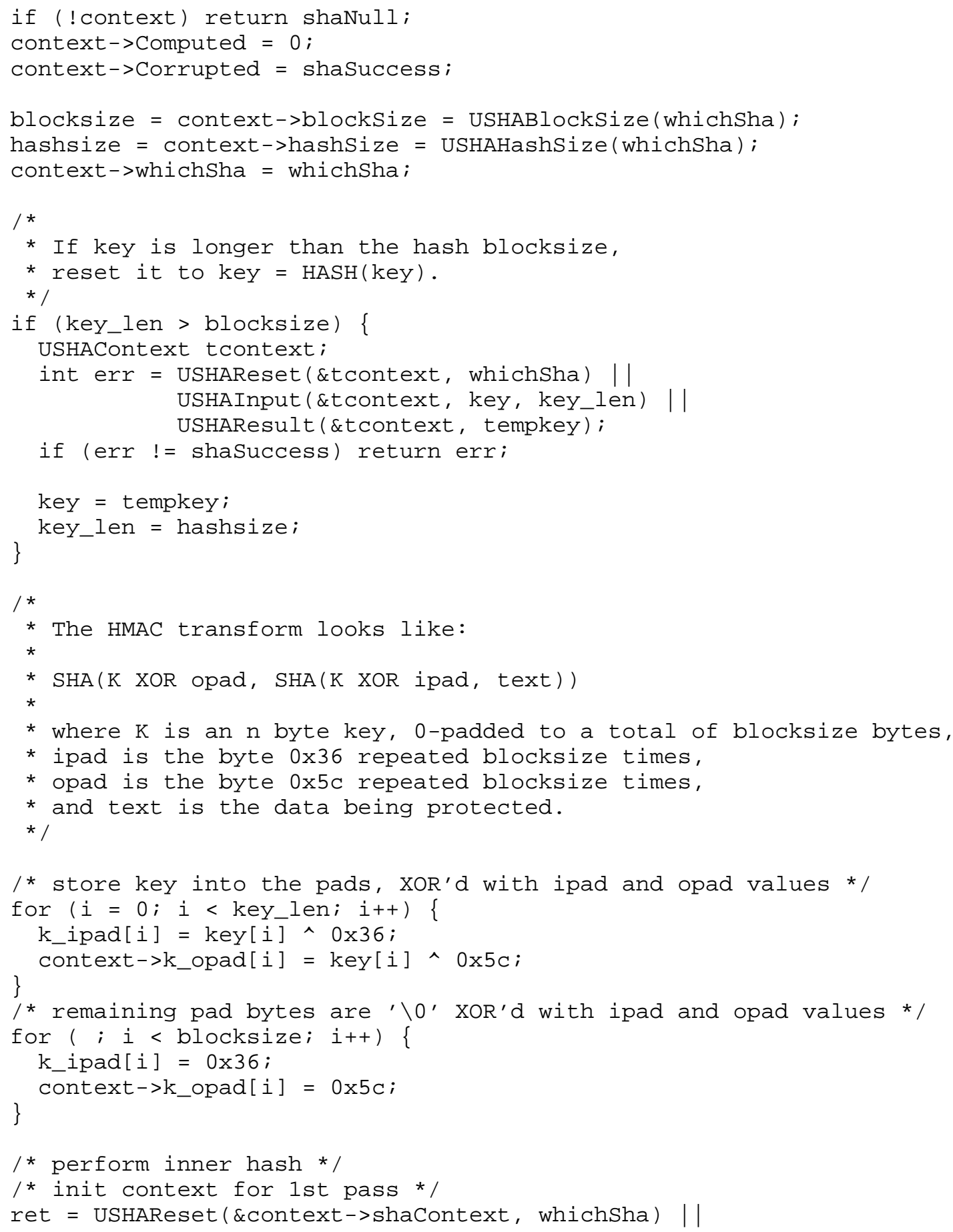




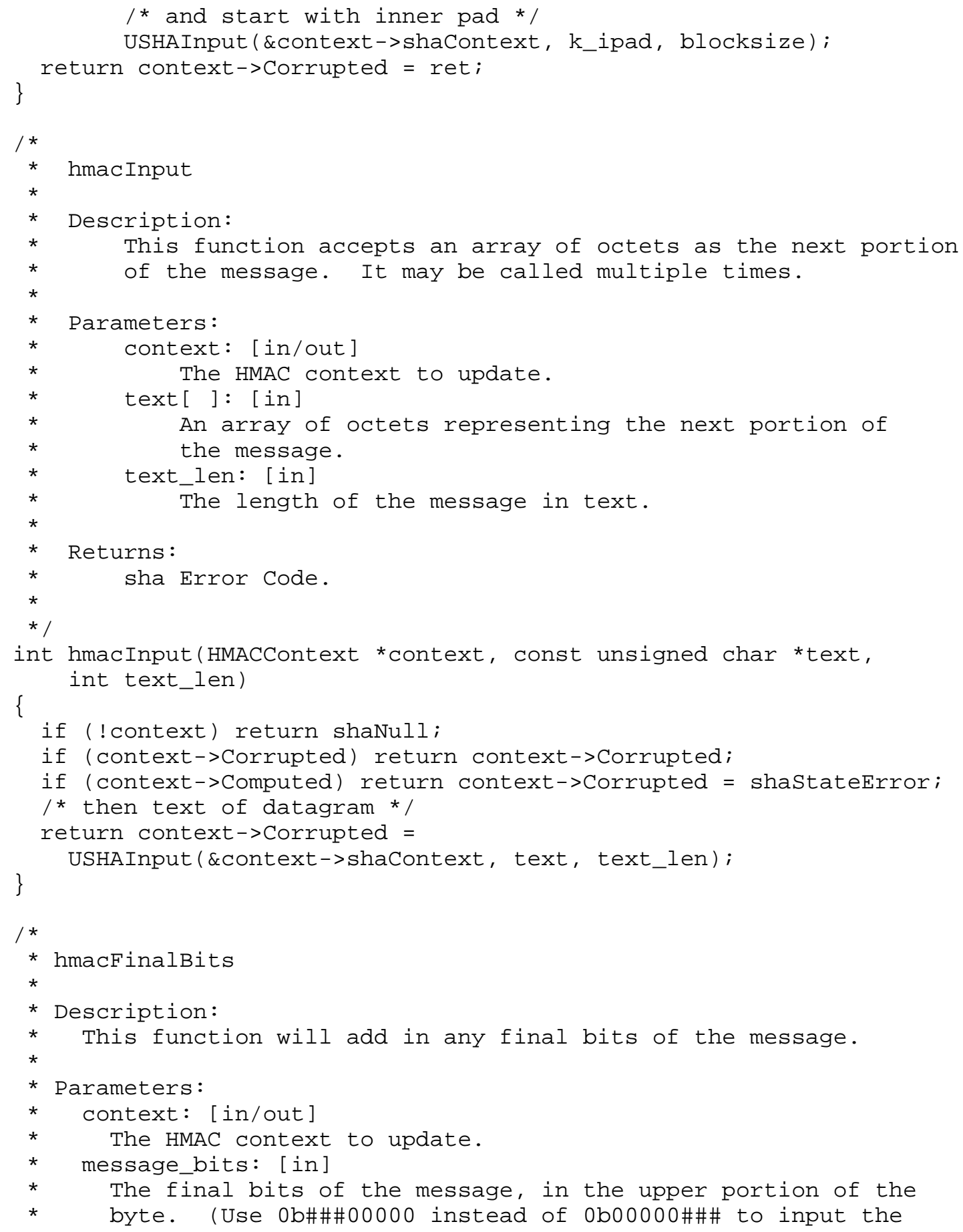




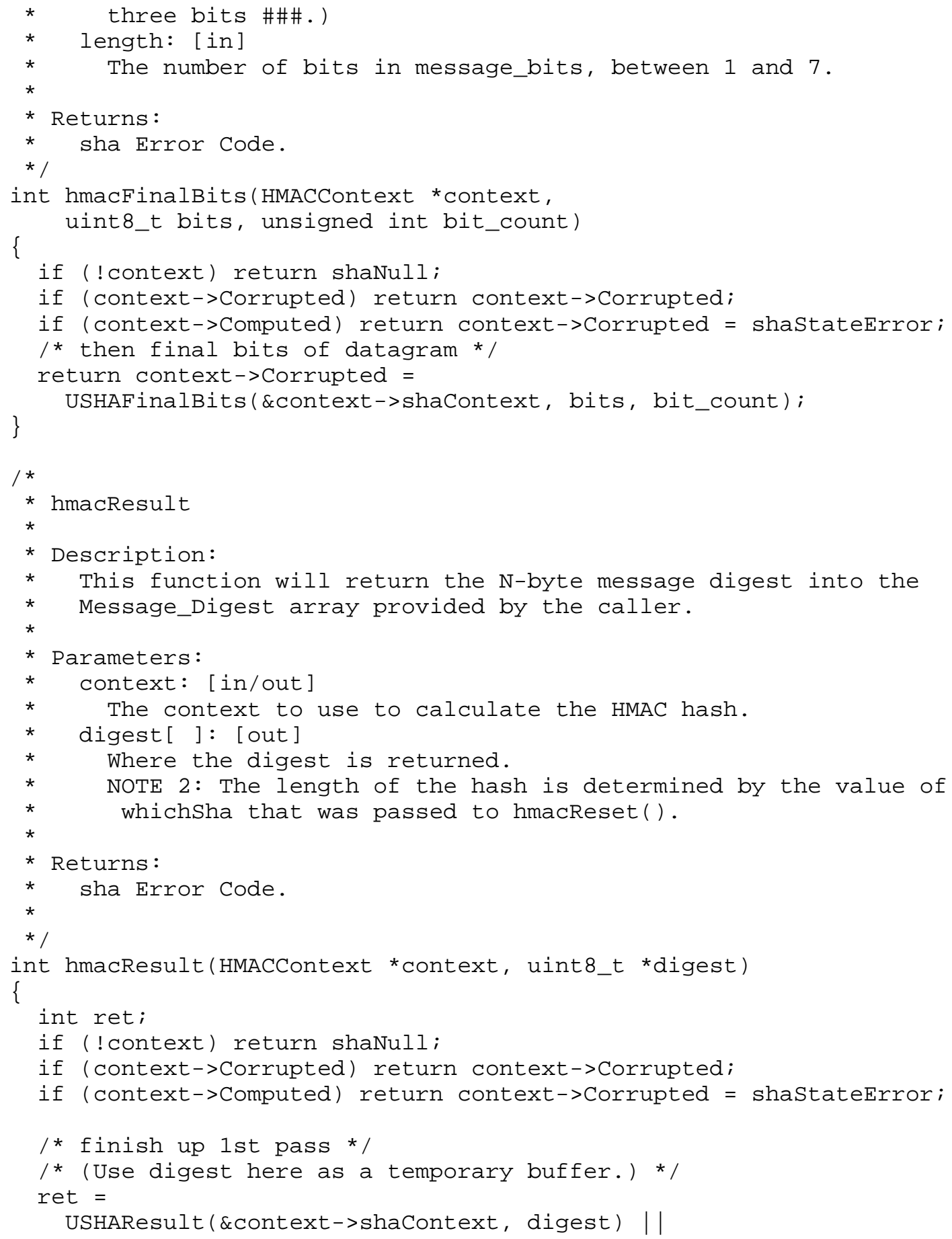




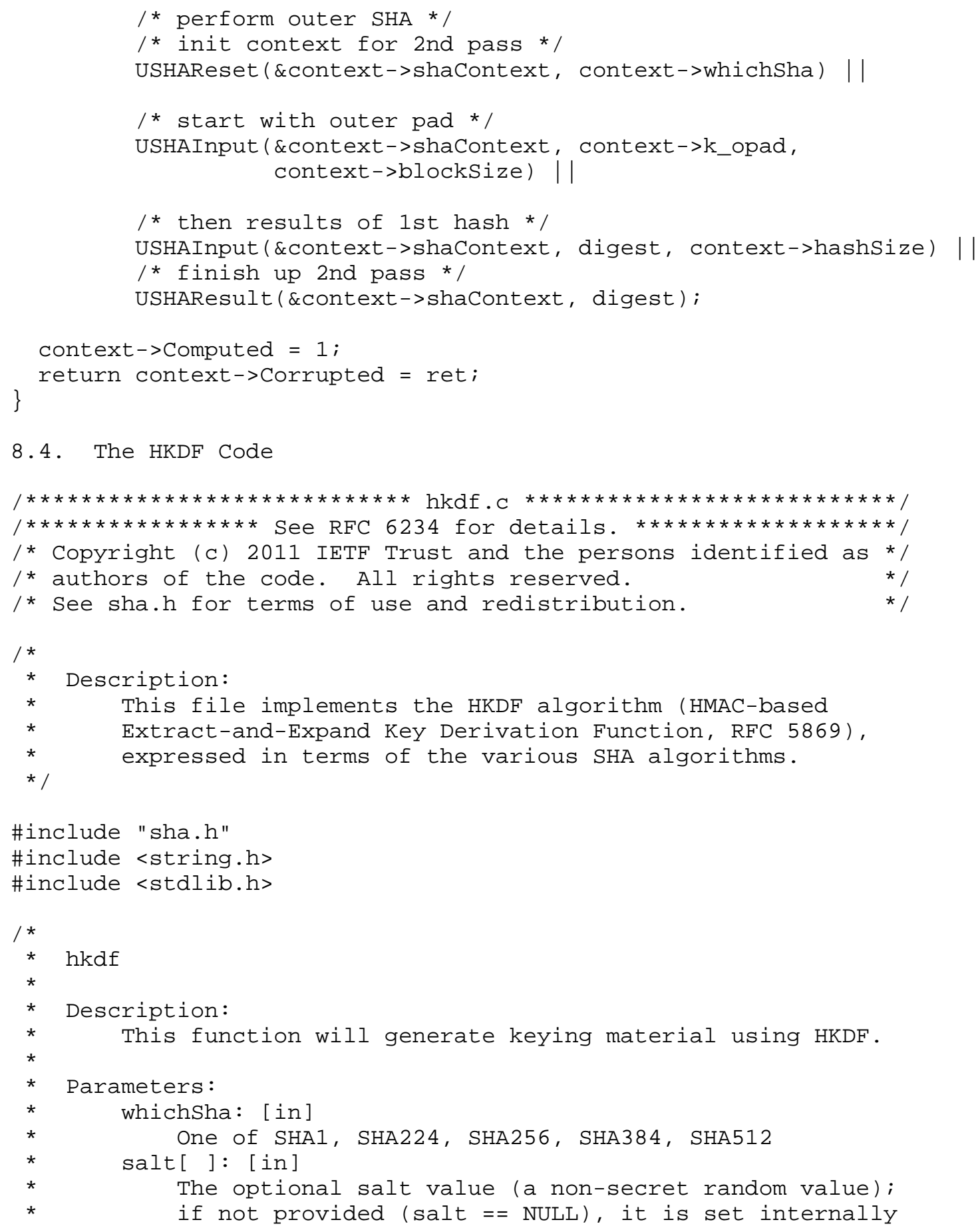




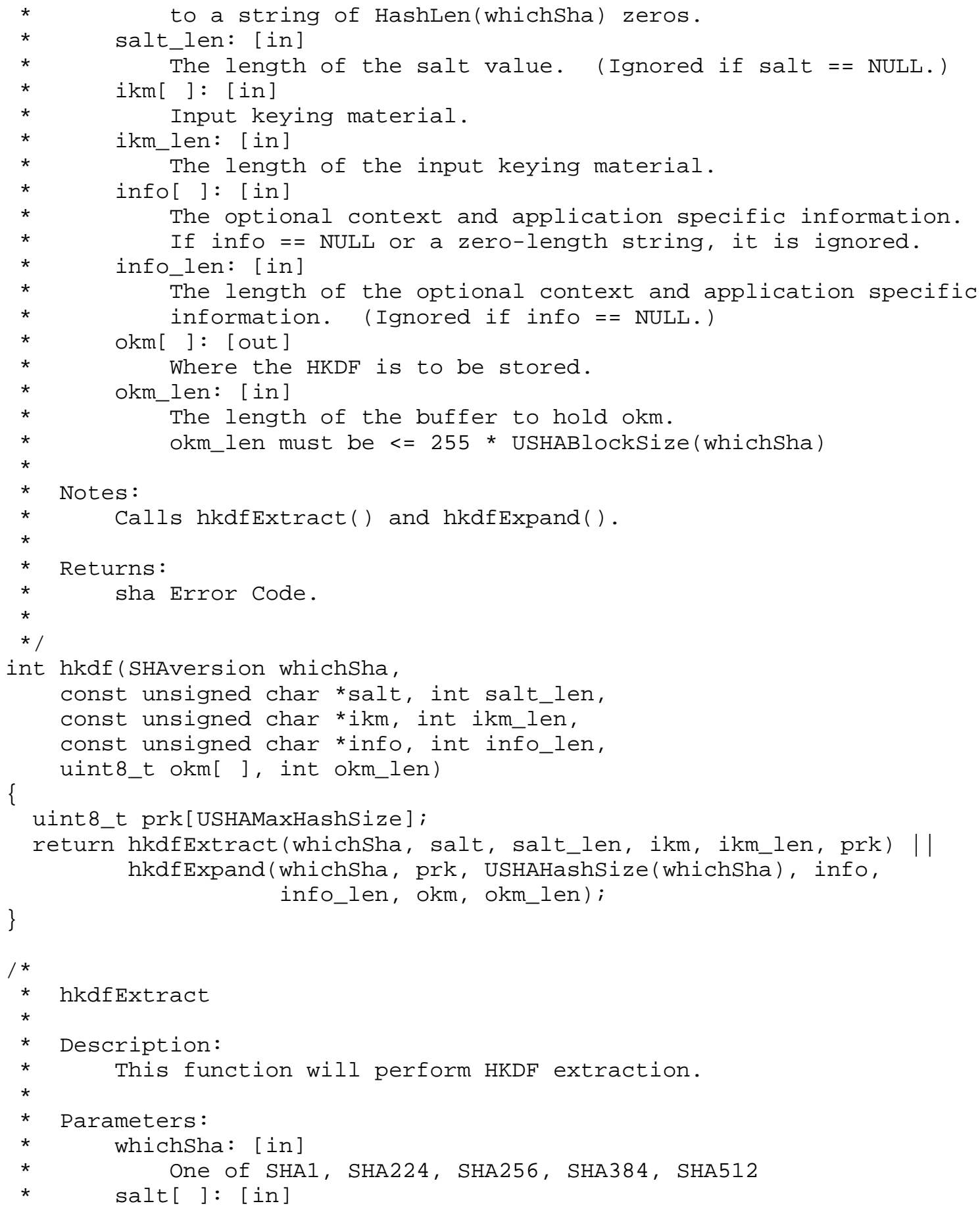




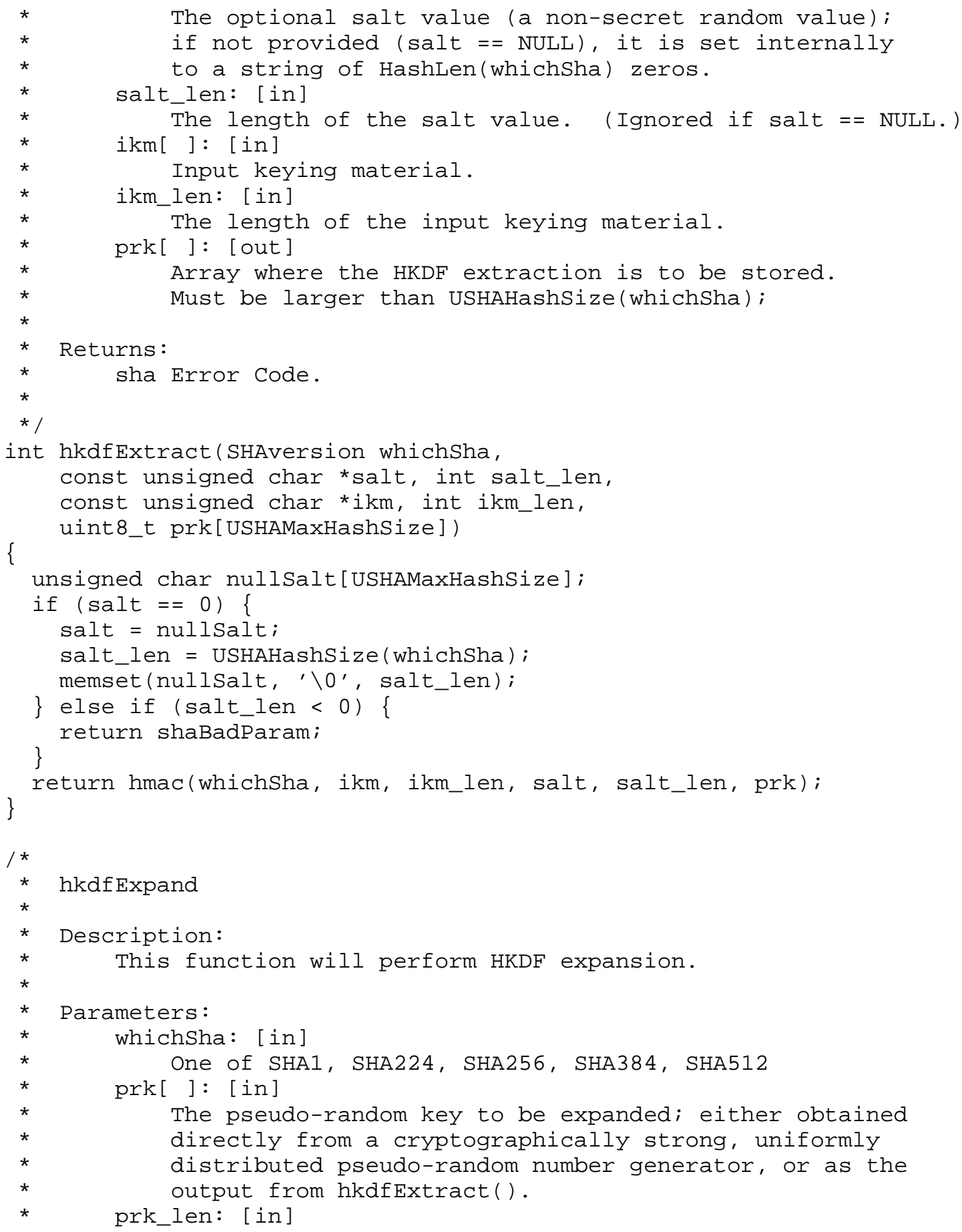




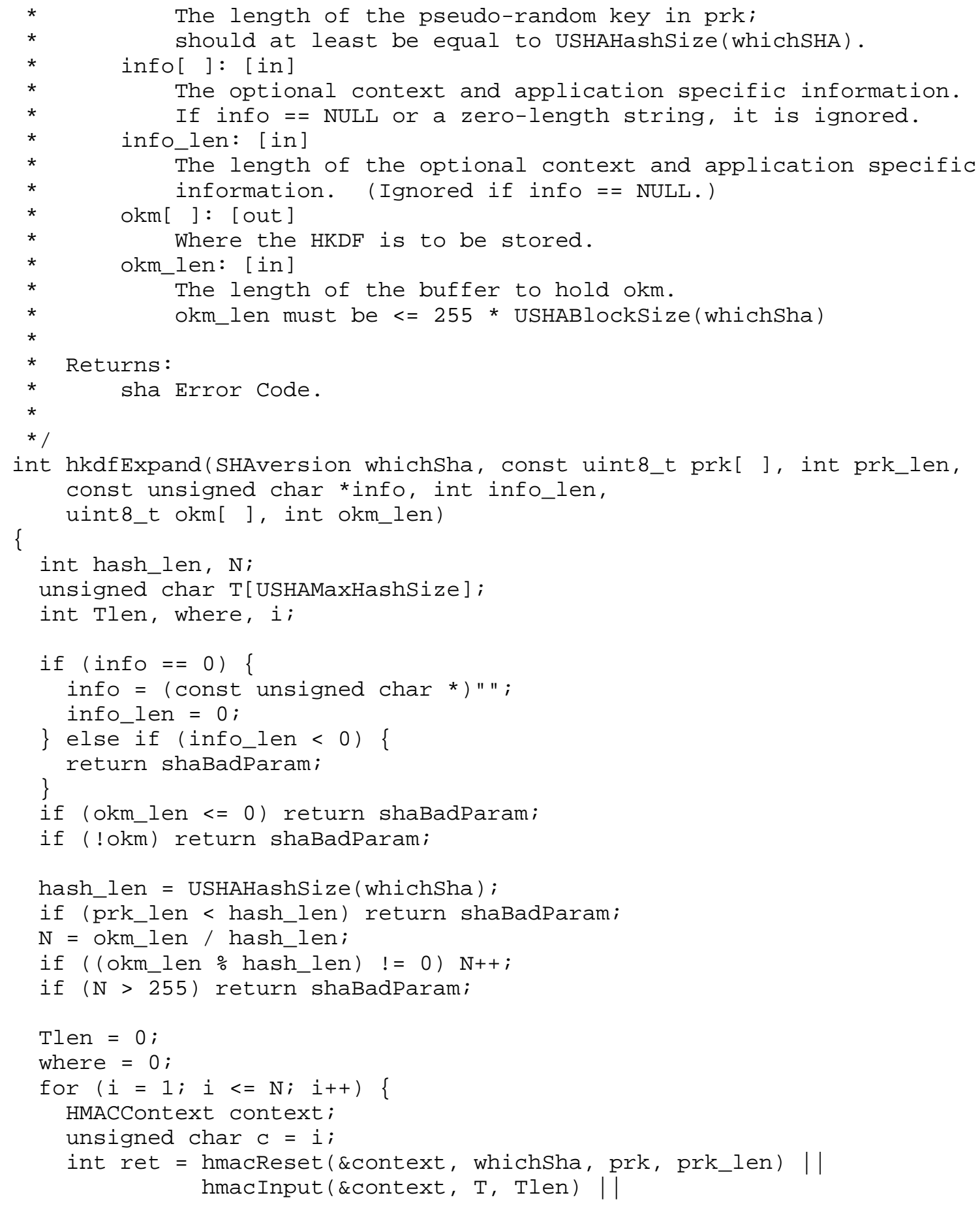




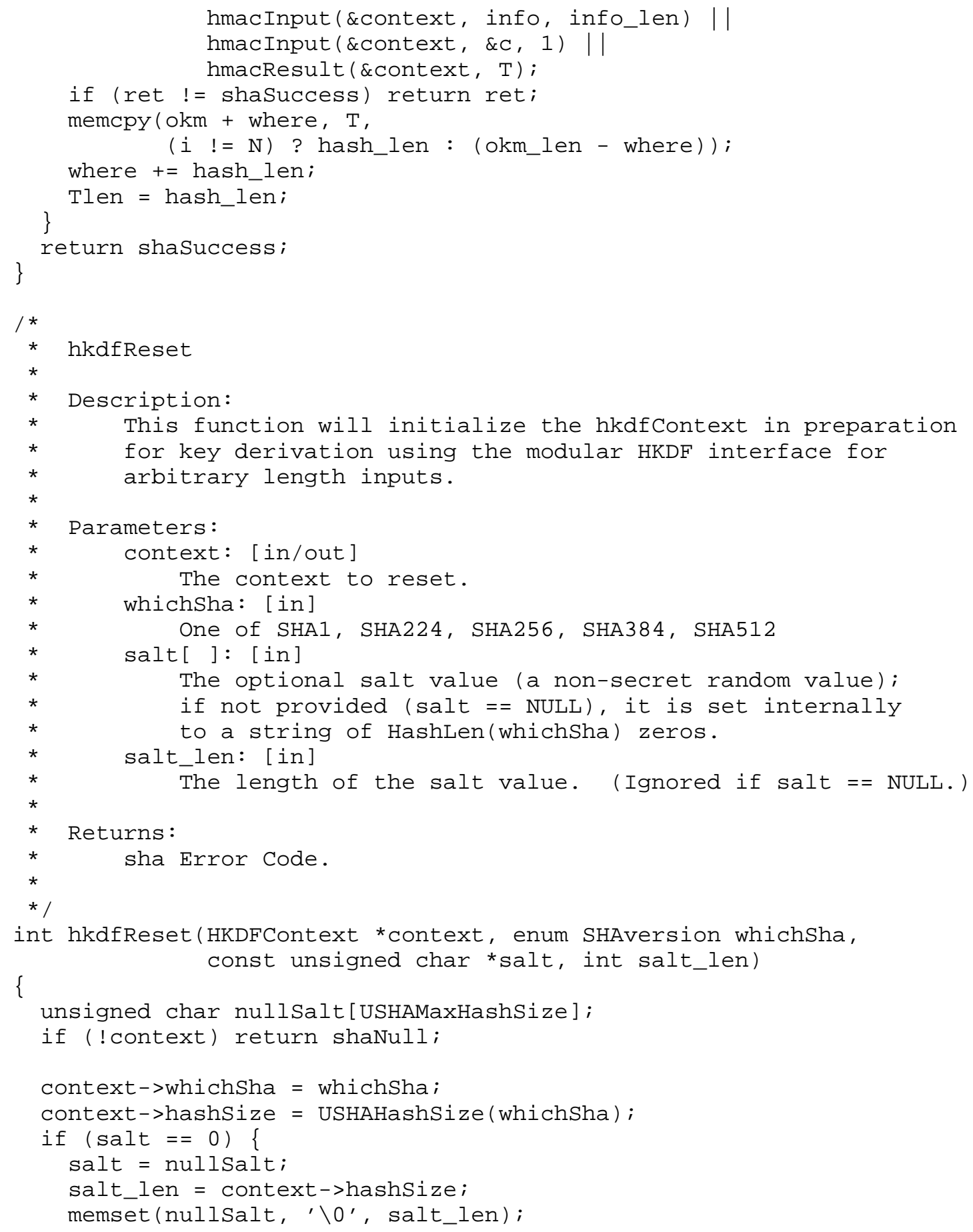




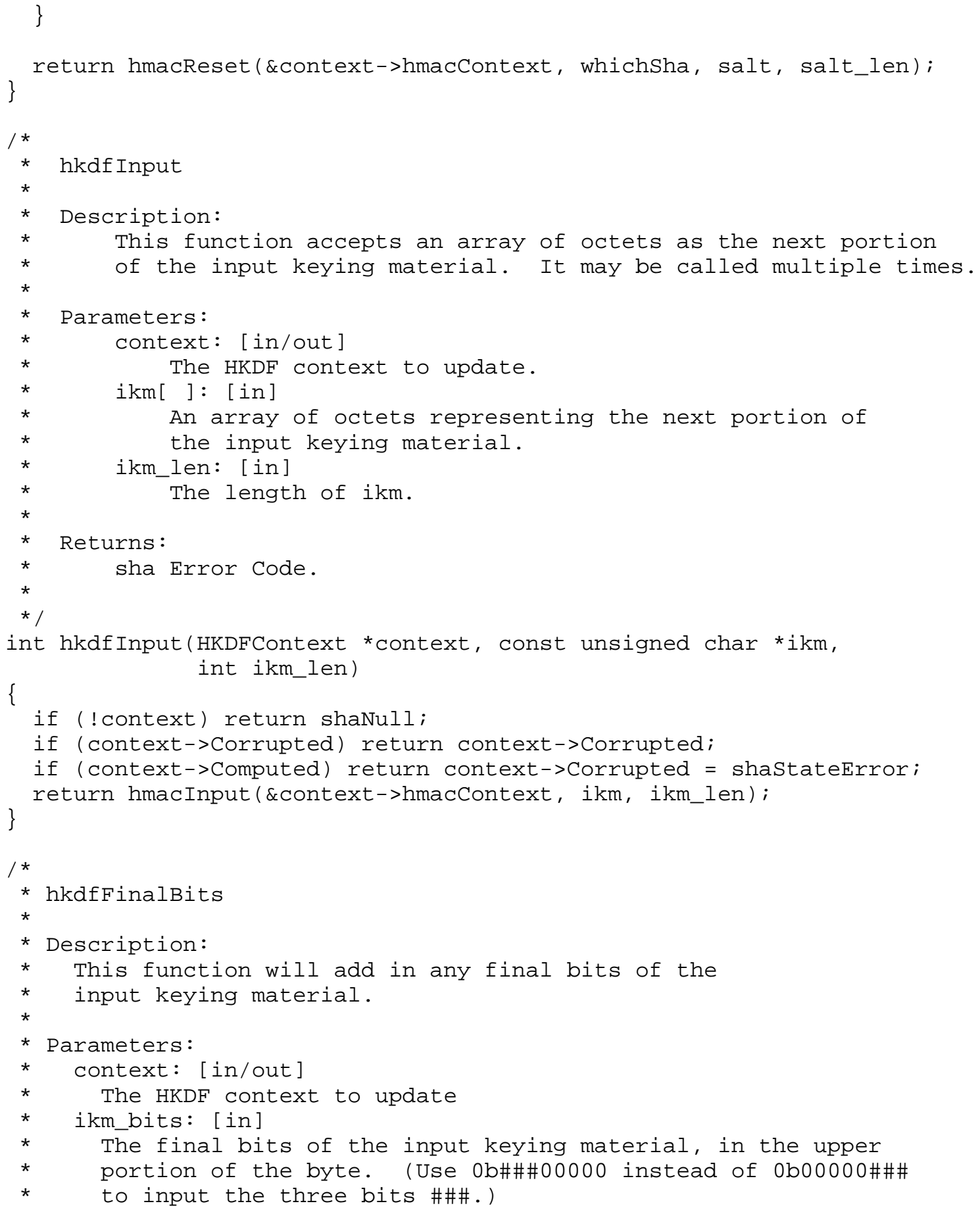




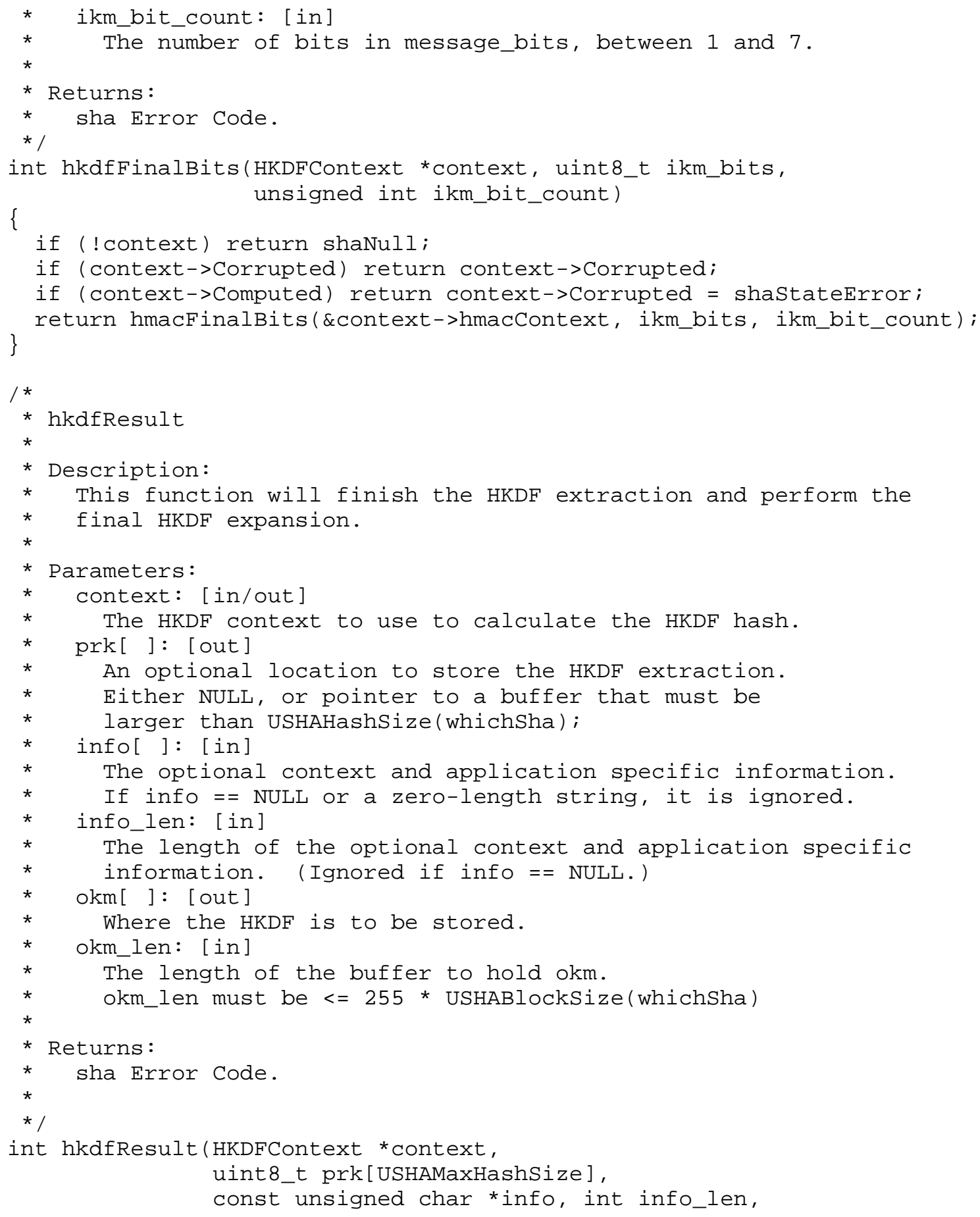


\{

uint8_t okm[ ], int okm_len)

uint8_t prkbuf[USHAMaxHashSize] ;

int ret;

if (!context) return shaNull;

if (context->Corrupted) return context->Corrupted;

if (context->Computed) return context->Corrupted $=$ shastateError;

if $($ !okm $)$ return context->Corrupted = shaBadParam;

if (!prk) prk = prkbuf;

ret $=$ hmacResult $(\&$ context $->$ hmacContext, prk) || hkdfExpand(context->whichSha, prk, context->hashsize, info, context->Computed $=1$; info_len, okm, okm_len); \}

return context->Corrupted = ret;

8.5. The Test Driver

The following code is a main program test driver to exercise the code in sha1.c, sha224-256.c, sha384-512.c, hmac.c, and hkdf.c. The test driver can also be used as a standalone program for generating the hashes. Note that the tests assume that character values are as in [US-ASCII] and a run time check warns if the code appears to have been compiled with some other character system.

See also [SHAVS].

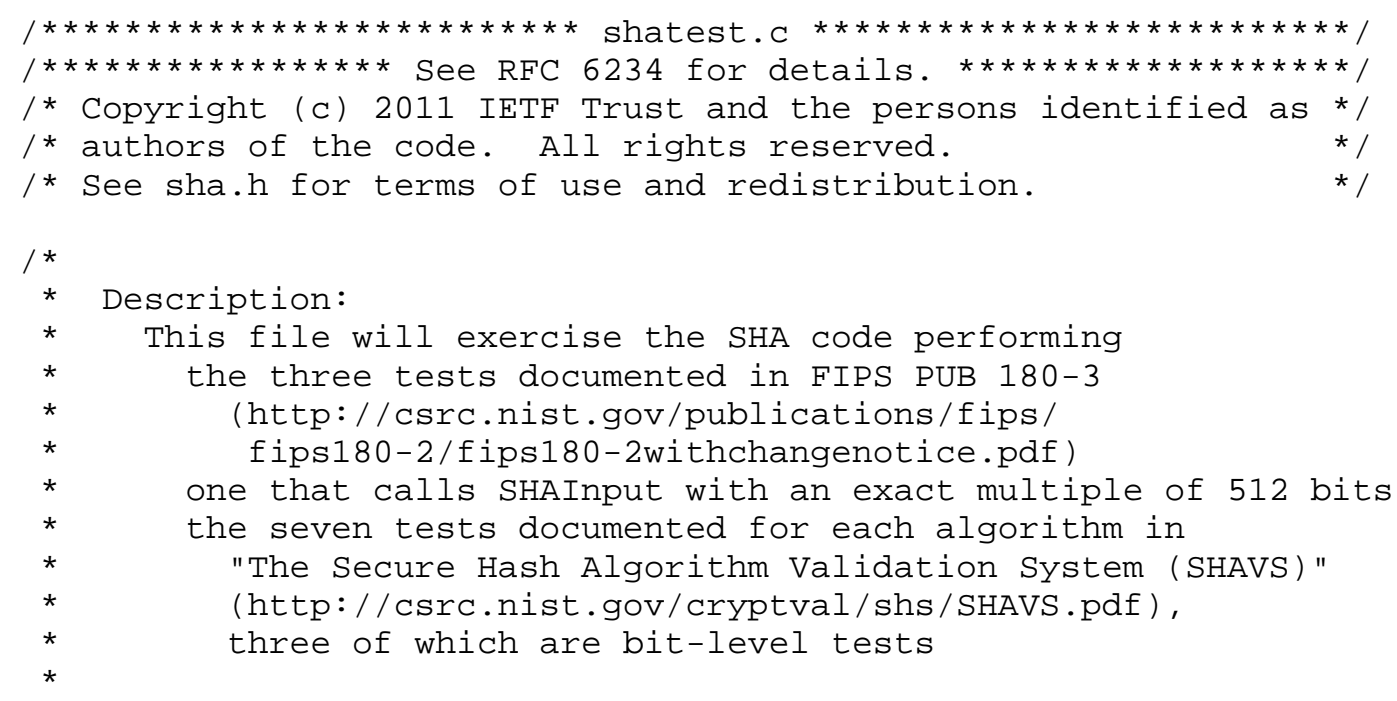




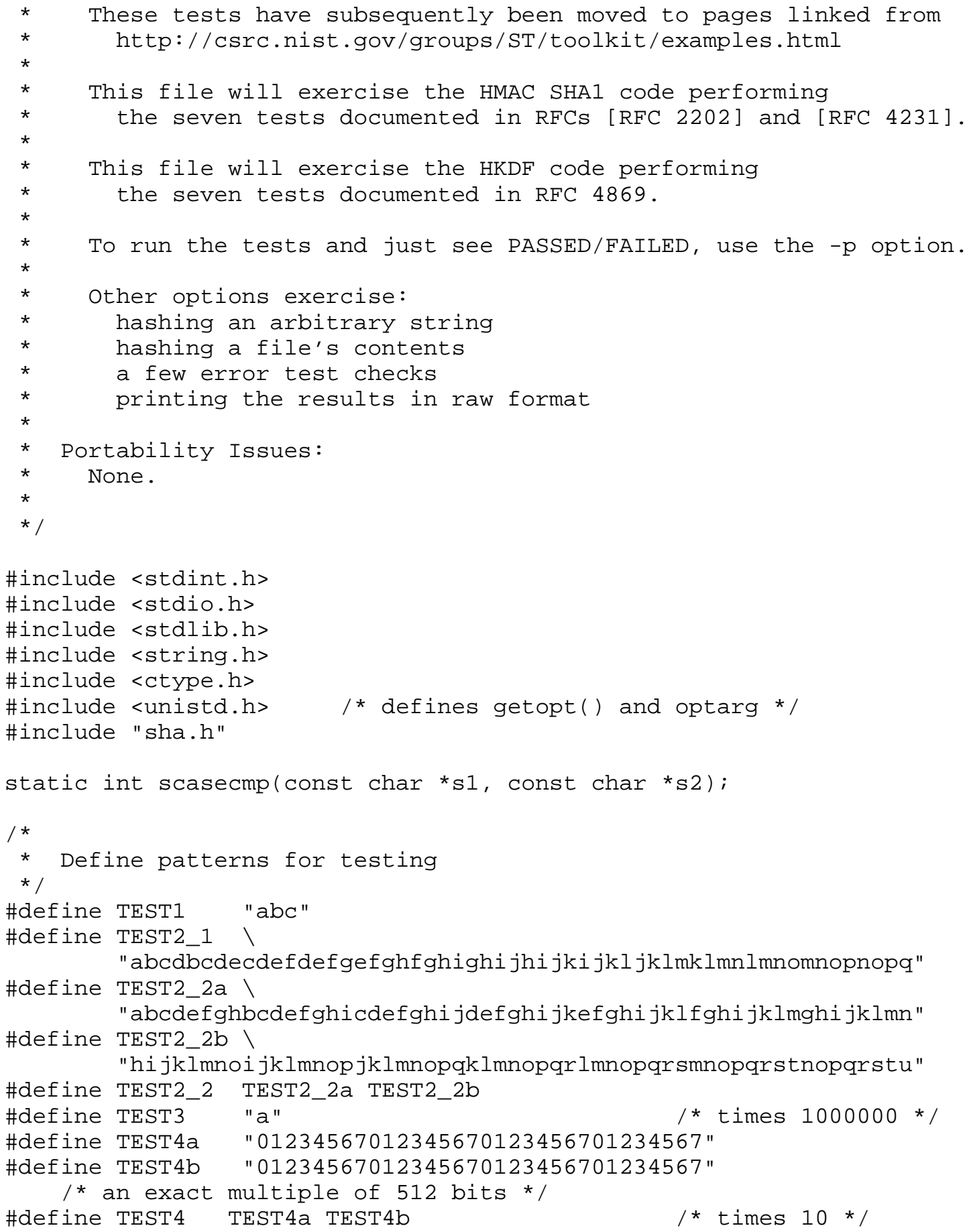


\#define TEST7_1

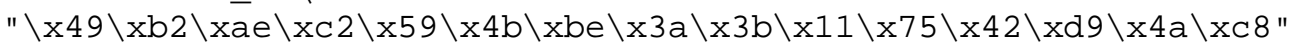
\#define TEST8_1 1

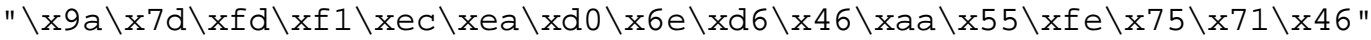
\#define TEST9_1 1

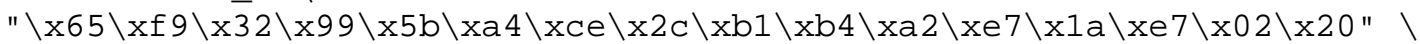

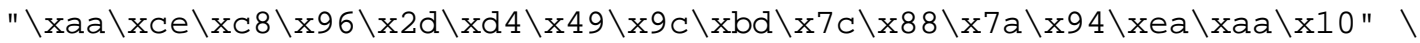

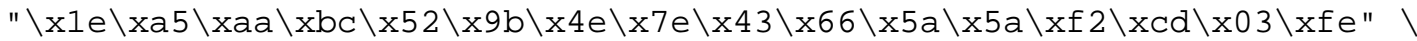

$" \backslash \mathrm{x} 67 \backslash \mathrm{x} 8 \mathrm{e} \backslash \mathrm{xa} 6 \backslash \mathrm{xa} 5 \backslash \mathrm{x} 00 \backslash \mathrm{x} 5 \mathrm{~b} \backslash \mathrm{xba} \backslash \mathrm{x} 3 \mathrm{~b} \backslash \mathrm{x} 08 \backslash \mathrm{x} 22 \backslash \mathrm{x} 04 \backslash \mathrm{xc} 2 \backslash \mathrm{x} 8 \mathrm{~b} \backslash \mathrm{x} 91 \backslash \mathrm{x} 09 \backslash \mathrm{xf} 4 " \backslash$

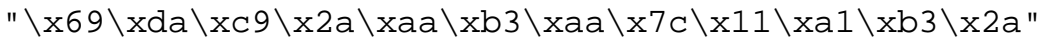

\#define TEST10_1

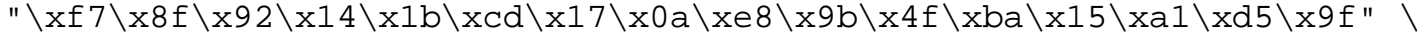

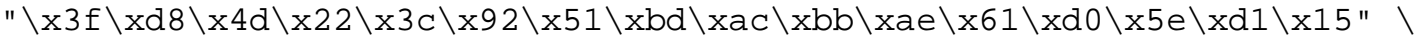

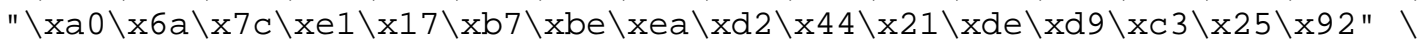

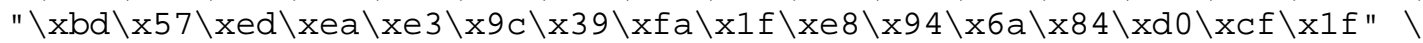

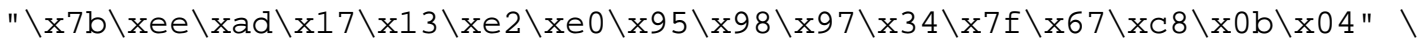

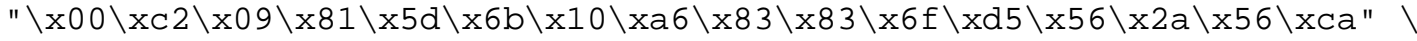

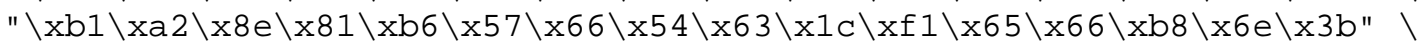

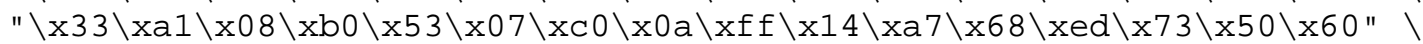

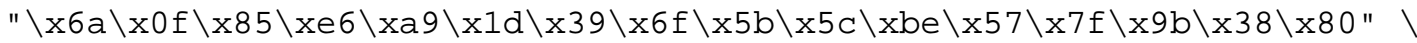

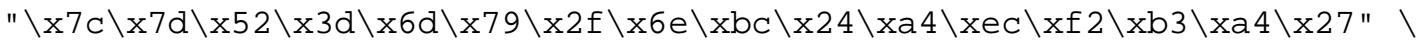

" $\backslash \mathrm{xcd} \backslash \mathrm{xbb} \backslash \mathrm{xfb}$ "

\#define TEST7_224

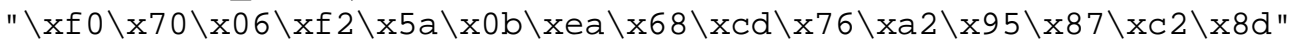

\#define TEST8_224 \}

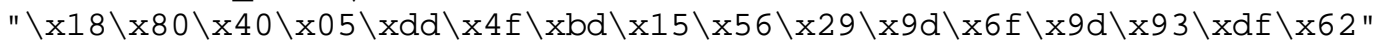

\#define TEST9_224

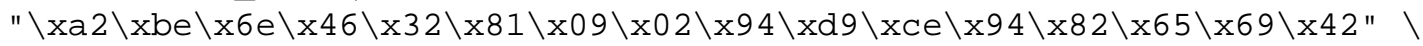

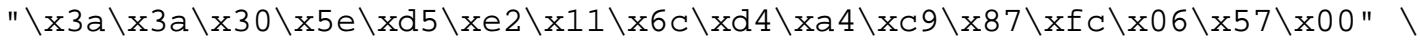

$" \backslash \mathrm{x} 64 \backslash \mathrm{x} 91 \backslash \mathrm{xb} 1 \backslash \mathrm{x} 49 \backslash \mathrm{xcc} \backslash \mathrm{xd} 4 \backslash \mathrm{xb} 5 \backslash \mathrm{x} 11 \backslash \mathrm{x} 30 \backslash \mathrm{xac} \backslash \mathrm{x} 62 \backslash \mathrm{xb} 1 \backslash \mathrm{x} 9 \mathrm{~d} \backslash \mathrm{xc} 2 \backslash \mathrm{x} 48 \backslash \mathrm{xc} 7 " \backslash$

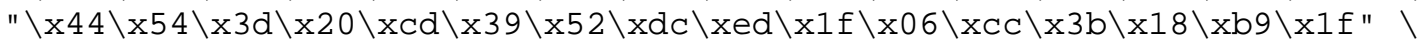

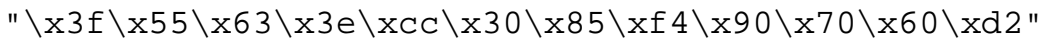

\#define TEST10_224

$" \backslash x 55 \backslash x b 2 \backslash x 10 \backslash x 07 \backslash x 9 c \backslash x 61 \backslash x b 5 \backslash x 3 a \backslash x d d \backslash x 52 \backslash x 06 \backslash x 22 \backslash x d 1 \backslash x a c \backslash x 97 \backslash x d 5 " \backslash$

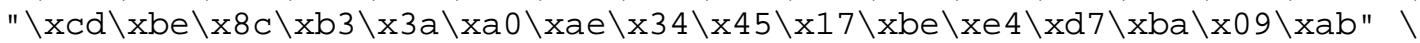

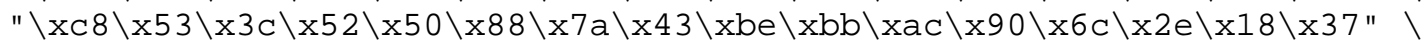

$" \backslash \mathrm{xf} 2 \backslash \mathrm{x} 6 \mathrm{~b} \backslash \mathrm{x} 36 \backslash \mathrm{xa} 5 \backslash \mathrm{x} 9 \mathrm{a} \backslash \mathrm{xe} 3 \backslash \mathrm{xbe} \backslash \mathrm{x} 78 \backslash \mathrm{x} 14 \backslash \mathrm{xd5} 5 \mathrm{x} 06 \backslash \mathrm{x} 89 \backslash \mathrm{x} 6 \mathrm{~b} \backslash \mathrm{x} 71 \backslash \mathrm{x} 8 \mathrm{~b} \backslash \mathrm{x} 2 \mathrm{a} " \backslash$

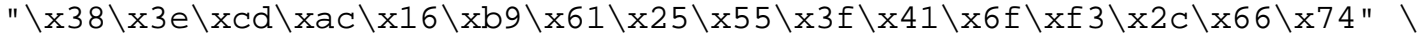

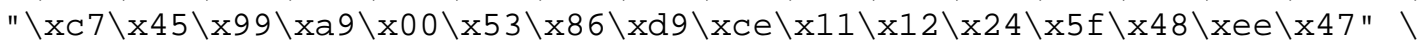

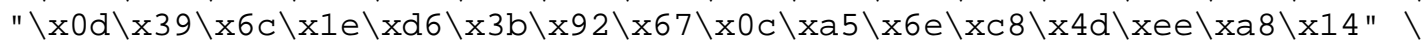

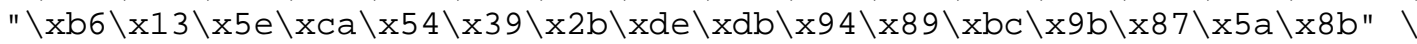

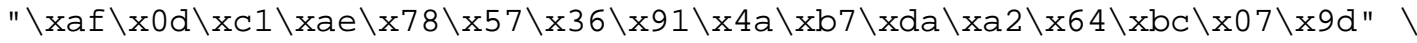

$" \backslash x 26 \backslash x 9 f \backslash x 2 c \backslash x 0 d \backslash x 7 e \backslash x d d \backslash x d 8 \backslash x 10 \backslash x a 4 \backslash x 26 \backslash x 14 \backslash x 5 a \backslash x 07 \backslash x 76 \backslash x f 6 \backslash x 7 c " \backslash$

"\x87\x82\x73"

\#define TEST7_256।

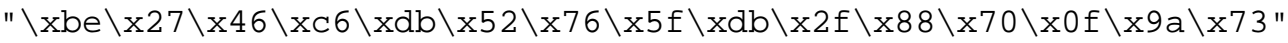

\#define TEST8_256

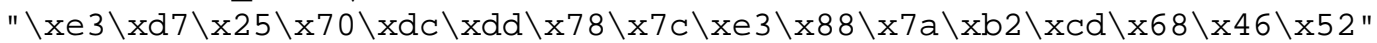


\#define TEST9_256 ।

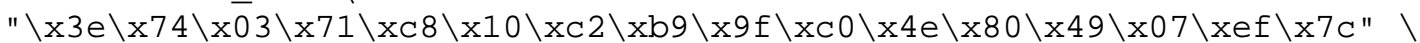

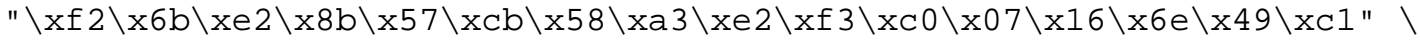

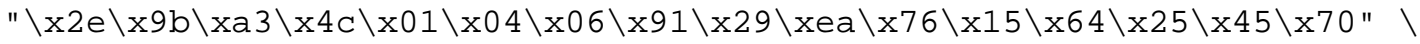

$" \backslash \mathrm{x} 3 a \backslash \mathrm{x} 2 \mathrm{~b} \backslash \mathrm{xd} \backslash \mathrm{x} 01 \backslash \mathrm{xe} 1 \backslash \mathrm{x} 6 \mathrm{x} \backslash \mathrm{xb} 0 \backslash \mathrm{xe} 0 \backslash \mathrm{x} 5 \mathrm{~d} \backslash \mathrm{xeb} \backslash \mathrm{xa} 0 \backslash \mathrm{x} 14 \backslash \mathrm{xeb} \backslash \mathrm{xff} \backslash \mathrm{x} 64 \backslash \mathrm{x} 06 " \backslash$

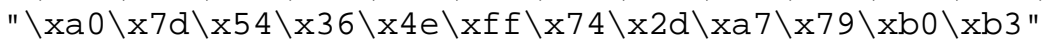

\#define TEST10_256 \}

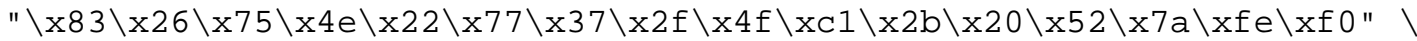

$" \backslash x 4 d \backslash x 8 a \backslash x 05 \backslash x 69 \backslash x 71 \backslash x b 1 \backslash x 1 a \backslash x d 5 \backslash x 71 \backslash x 23 \backslash x a 7 \backslash x c 1 \backslash x 37 \backslash x 76 \backslash x 00 \backslash x 00 " \backslash$

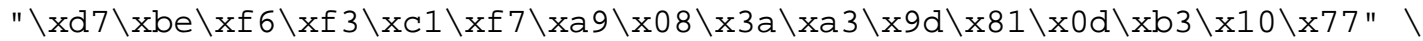

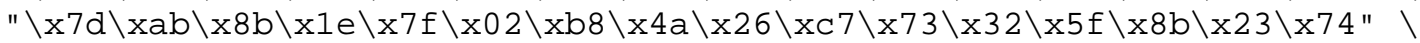

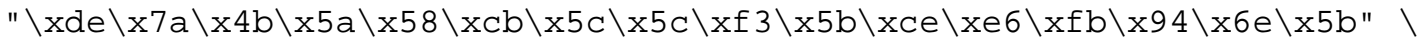

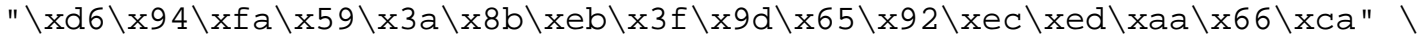

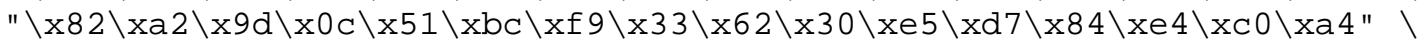

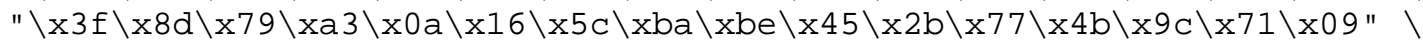

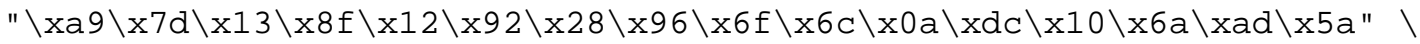

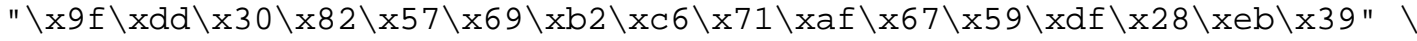

" $\backslash \mathrm{x} 3 \mathrm{~d} \backslash \mathrm{x} 54 \backslash \mathrm{xd} 6$ "

\#define TEST7_384 ।

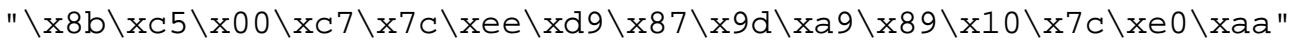

\#define TEST8_384

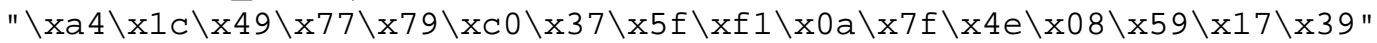

\#define TEST9_384

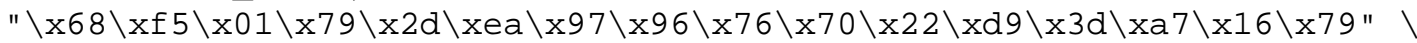

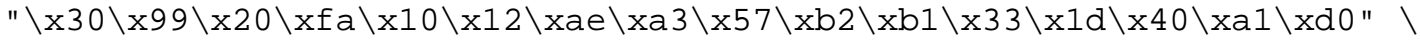

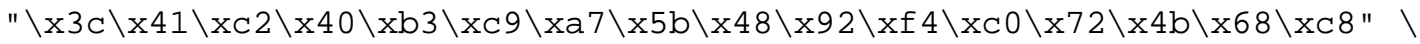

$" \backslash x 75 \backslash x 32 \backslash x 1 a \backslash x b 8 \backslash x c f \backslash x e 5 \backslash x 02 \backslash x 3 b \backslash x d 3 \backslash x 75 \backslash x b c \backslash x 0 f \backslash x 94 \backslash x b d \backslash x 89 \backslash x f e " \backslash$

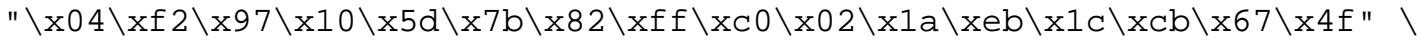

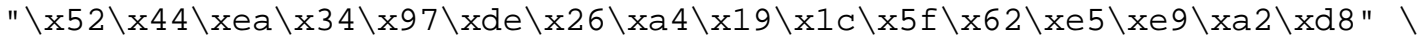

$" \backslash x 08 \backslash x 2 f \backslash x 05 \backslash x 51 \backslash x f 4 \backslash x a 5 \backslash x 30 \backslash x 68 \backslash x 26 \backslash x e 9 \backslash x 1 c \backslash x c 0 \backslash x 06 \backslash x c e \backslash x 1 b \backslash x f 6 " \backslash$

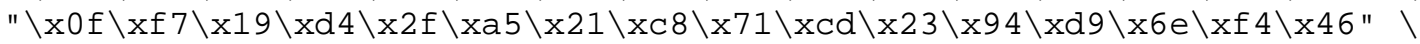

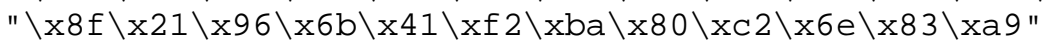

\#define TEST10_384

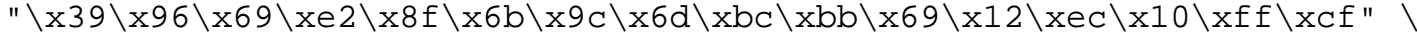

$" \backslash x 74 \backslash x 79 \backslash x 03 \backslash x 49 \backslash x b 7 \backslash x d c \backslash x 8 f \backslash x b e \backslash x 4 a \backslash x 8 e \backslash x 7 b \backslash x 3 b \backslash x 56 \backslash x 21 \backslash x d b \backslash x 0 f " \backslash$

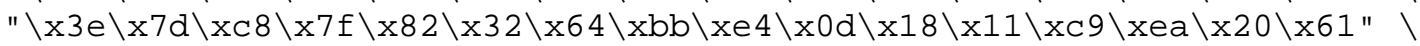

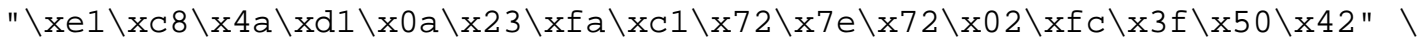

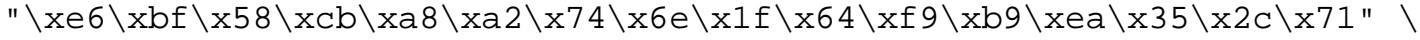

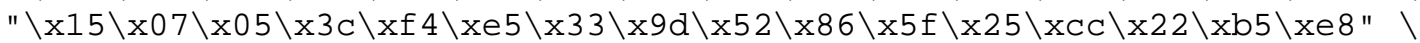

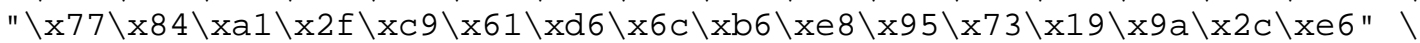

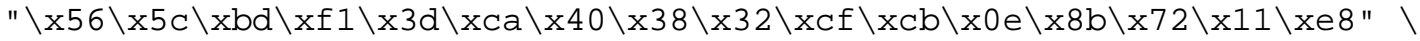

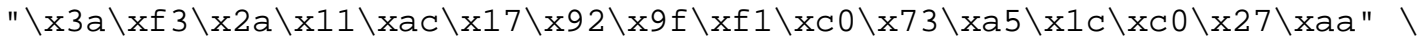

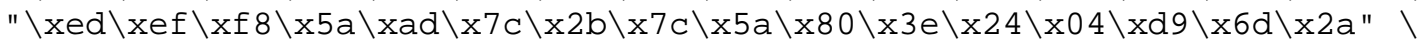

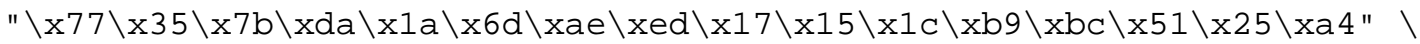

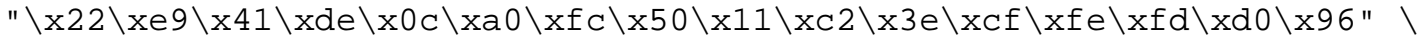

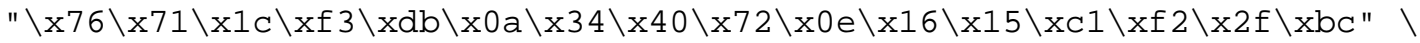

$" \backslash x 3 c \backslash x 72 \backslash x 1 d \backslash x e 5 \backslash x 21 \backslash x e 1 \backslash x b 9 \backslash x 9 b \backslash x a 1 \backslash x b d \backslash x 55 \backslash x 77 \backslash x 40 \backslash x 86 \backslash x 42 \backslash x 14 " \backslash$

$" \backslash x 7 e \backslash x d 0 \backslash x 96 "$ 
\#define TEST7_512 \}

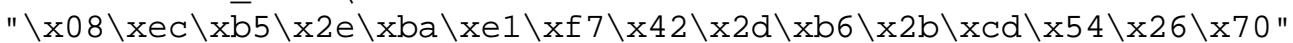
\#define TEST8_512 \

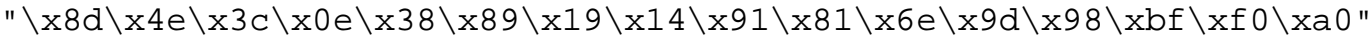
\#define TEST9_512 \

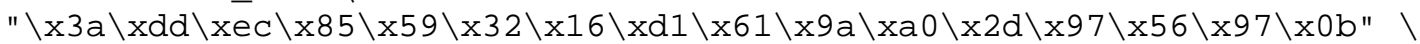

$" \backslash x f c \backslash x 70 \backslash x a c \backslash x e 2 \backslash x 74 \backslash x 4 f \backslash x 7 c \backslash x 6 b \backslash x 27 \backslash x 88 \backslash x 15 \backslash x 10 \backslash x 28 \backslash x f 7 \backslash x b 6 \backslash x a 2 " \backslash$

$" \backslash \mathrm{x} 55 \backslash \mathrm{x} 0 \mathrm{f} \backslash \mathrm{xd} \backslash \backslash \mathrm{x} 4 \mathrm{a} \backslash \mathrm{x} 7 \mathrm{e} \backslash \mathrm{x} 6 \mathrm{e} \backslash \mathrm{x} 69 \backslash \mathrm{xc} 2 \backslash \mathrm{xc} 9 \backslash \mathrm{xb} 4 \backslash \mathrm{x} 5 \mathrm{f} \backslash \mathrm{xc} 4 \backslash \mathrm{x} 54 \backslash \mathrm{x} 96 \backslash \mathrm{x} 6 \mathrm{~d} \backslash \mathrm{xc} 3 " \backslash$

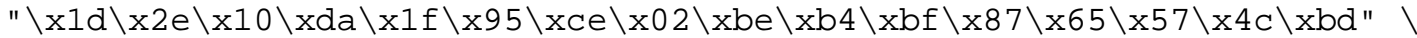

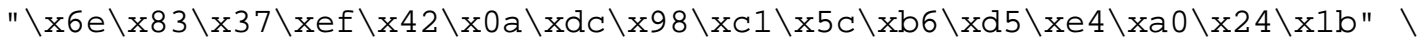

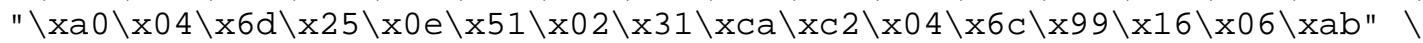

$" \backslash x 4 e \backslash x e 4 \backslash x 14 \backslash x 5 b \backslash x e e \backslash x 2 f \backslash x f 4 \backslash x b b \backslash x 12 \backslash x 3 a \backslash x a b \backslash x 49 \backslash x 8 d \backslash x 9 d \backslash x 44 \backslash x 79 " \backslash$

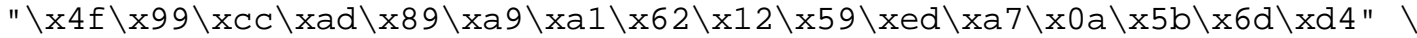

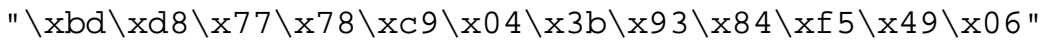

\#define TEST10_512 \

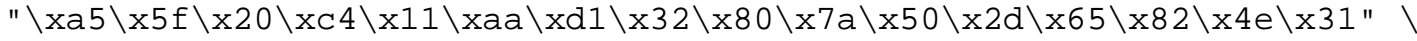

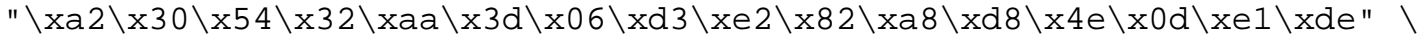

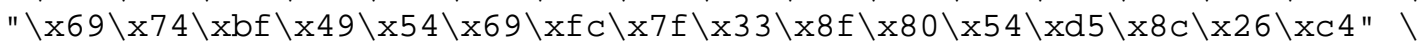

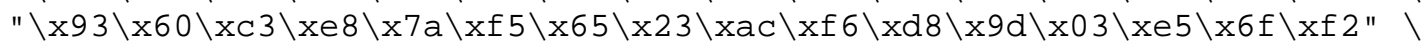

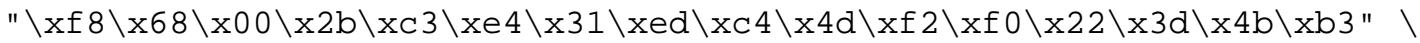

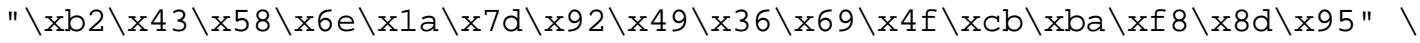

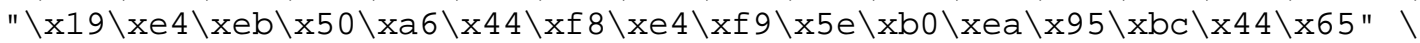

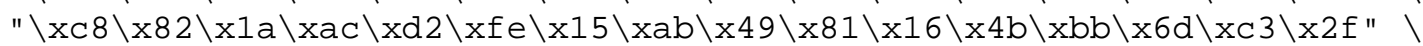

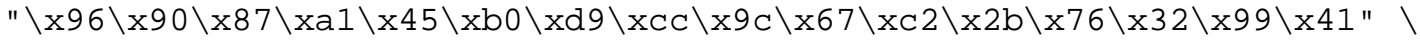

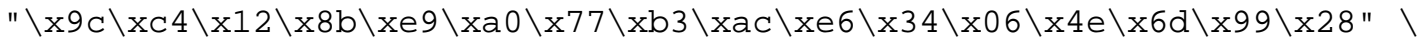

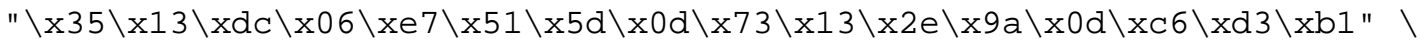

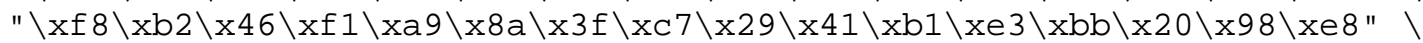

$" \backslash \mathrm{xbf} \backslash \mathrm{x} 16 \backslash \mathrm{xf} 2 \backslash \mathrm{x} 68 \backslash \mathrm{xd6} \backslash \mathrm{x} 4 \mathrm{f} \backslash \mathrm{x} 0 \mathrm{~b} \backslash \mathrm{x} 0 \mathrm{f} \backslash \mathrm{x} 47 \backslash \mathrm{x} 07 \backslash \mathrm{xfe} \backslash \mathrm{x} 1 \mathrm{e} \backslash \mathrm{xa} 1 \backslash \mathrm{xa} 1 \backslash \mathrm{x} 79 \backslash \mathrm{x} 1 \mathrm{~b} " \backslash$

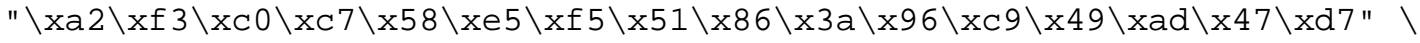

" $\backslash \mathrm{xfb} \backslash \mathrm{x} 40 \backslash \mathrm{xd} 2$ "

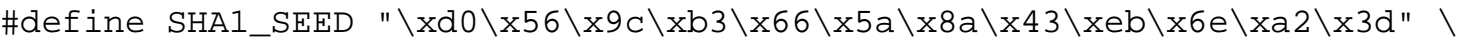

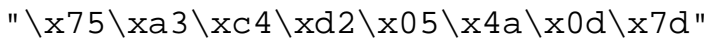

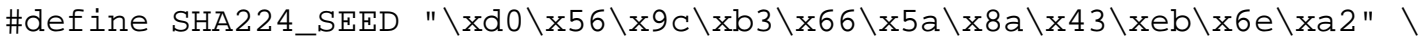

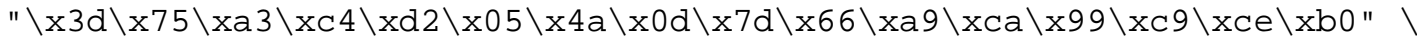

" $\backslash \times 27 "$

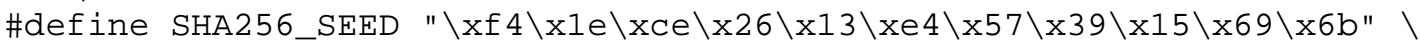

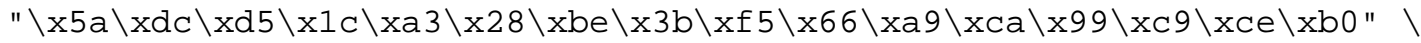

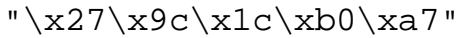

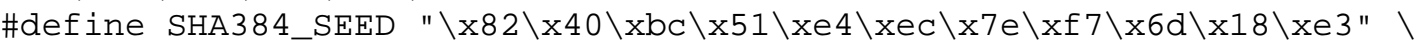

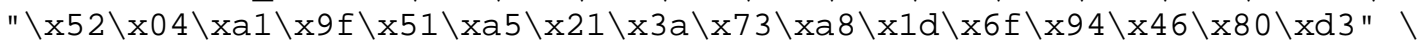

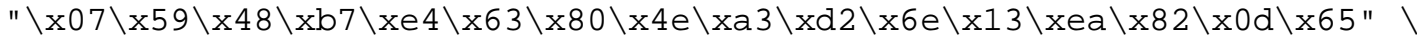

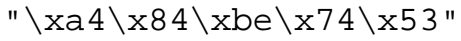

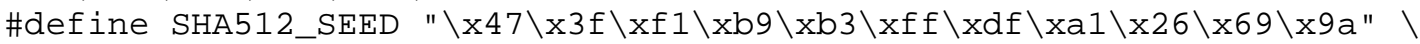

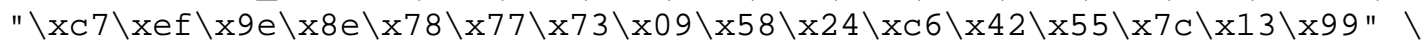

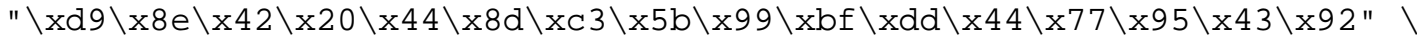

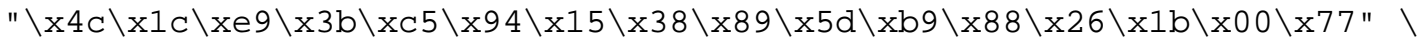

$" \backslash x 4 b \backslash x 12 \backslash x 27 \backslash x 20 \backslash x 39 "$ 


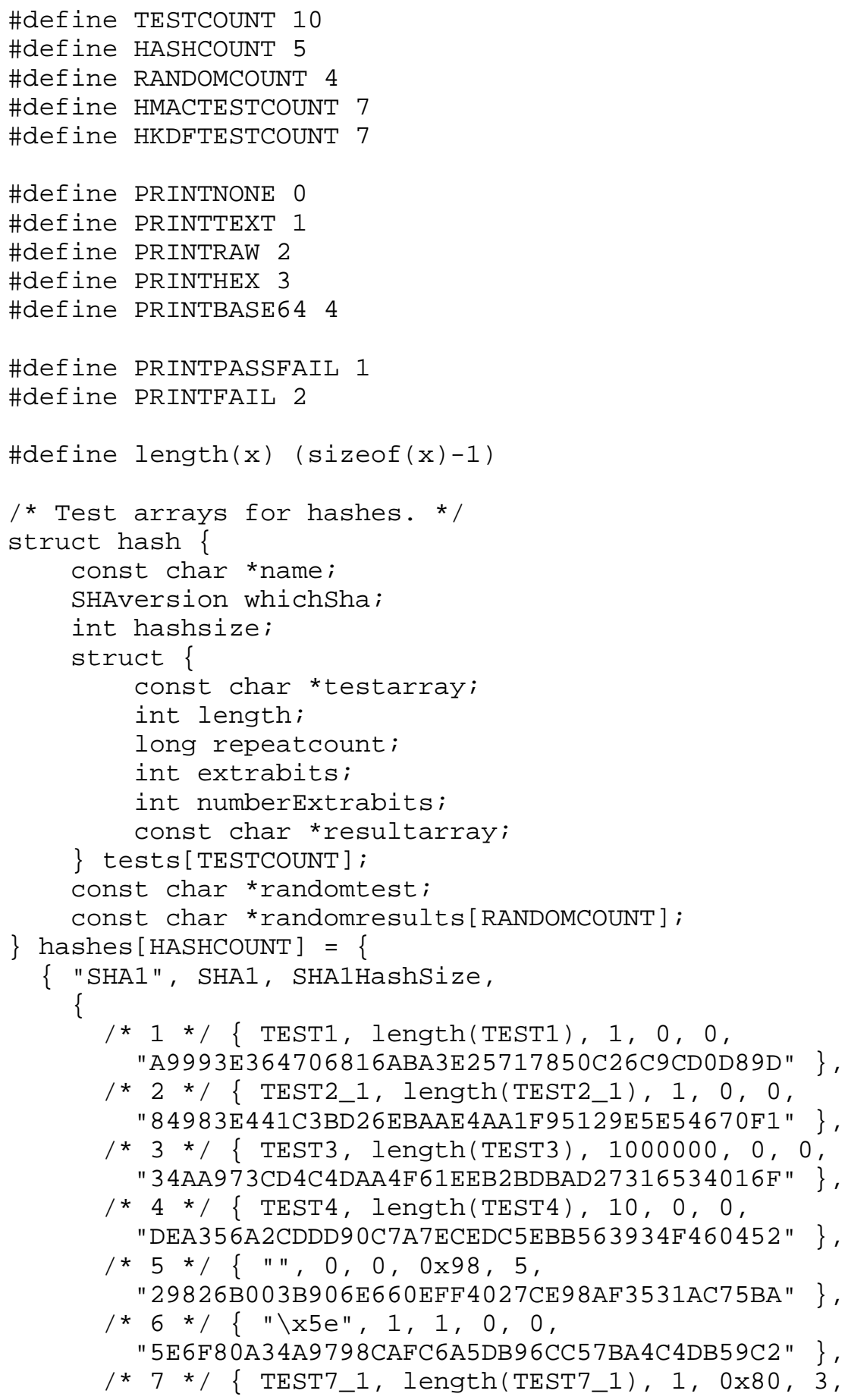




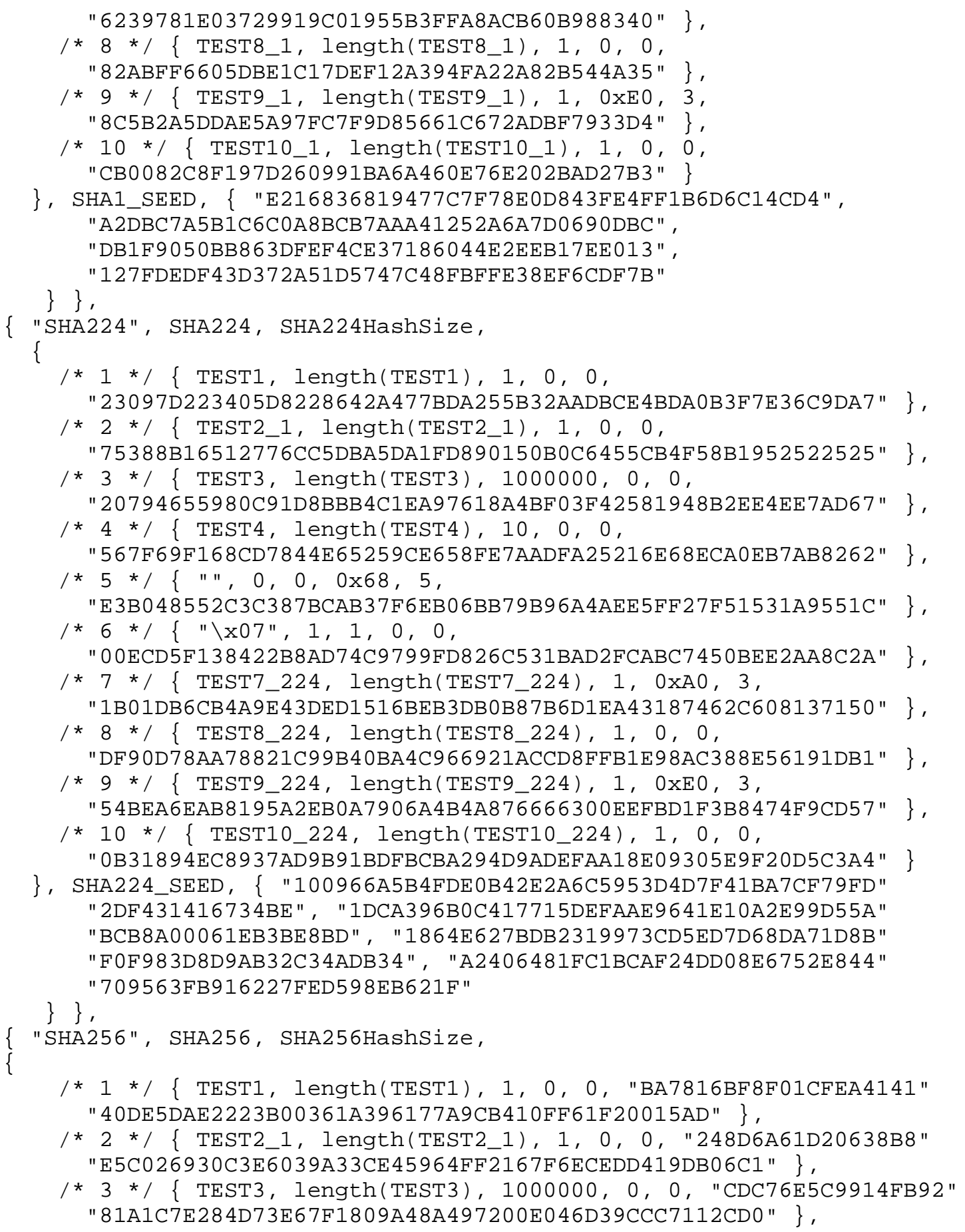




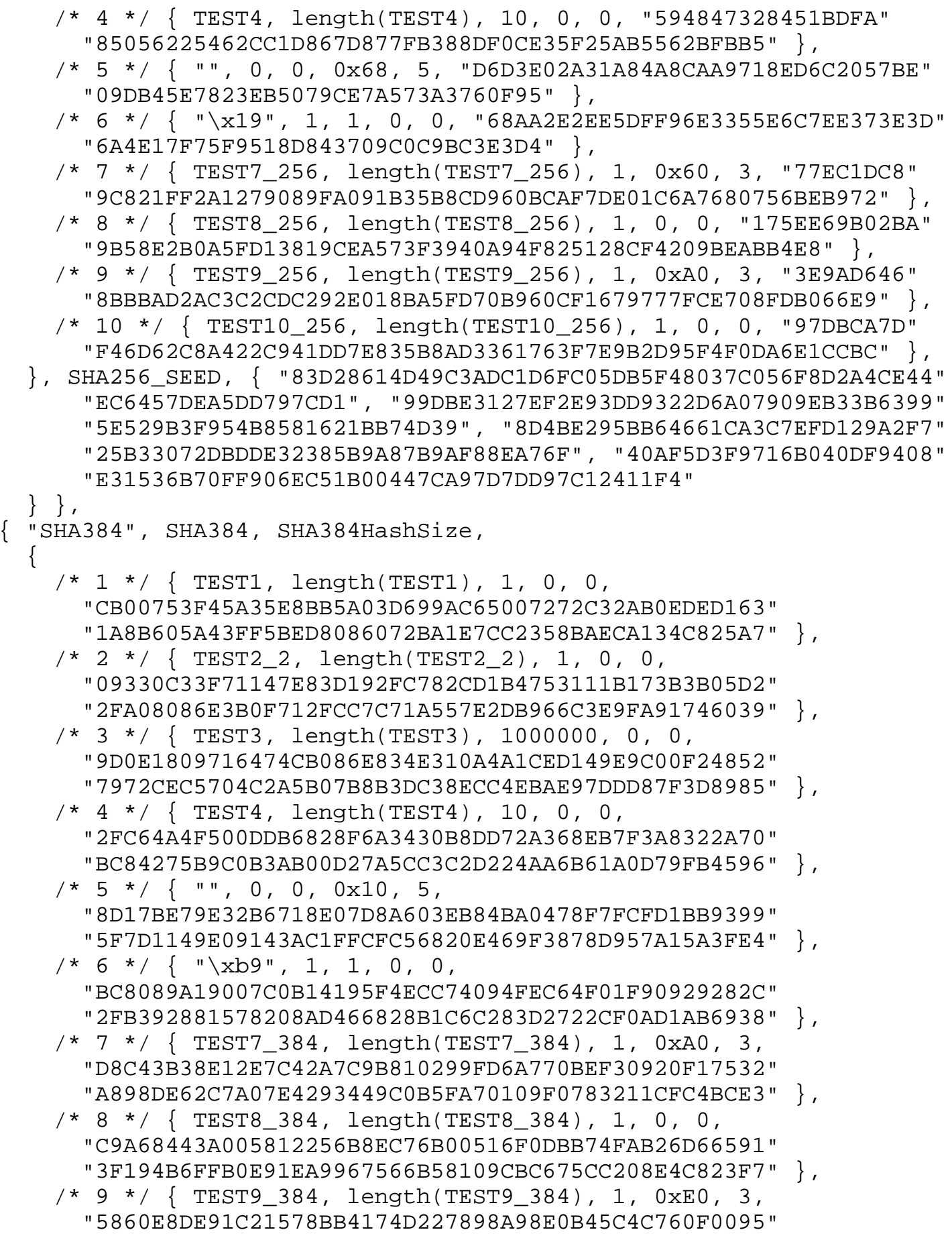




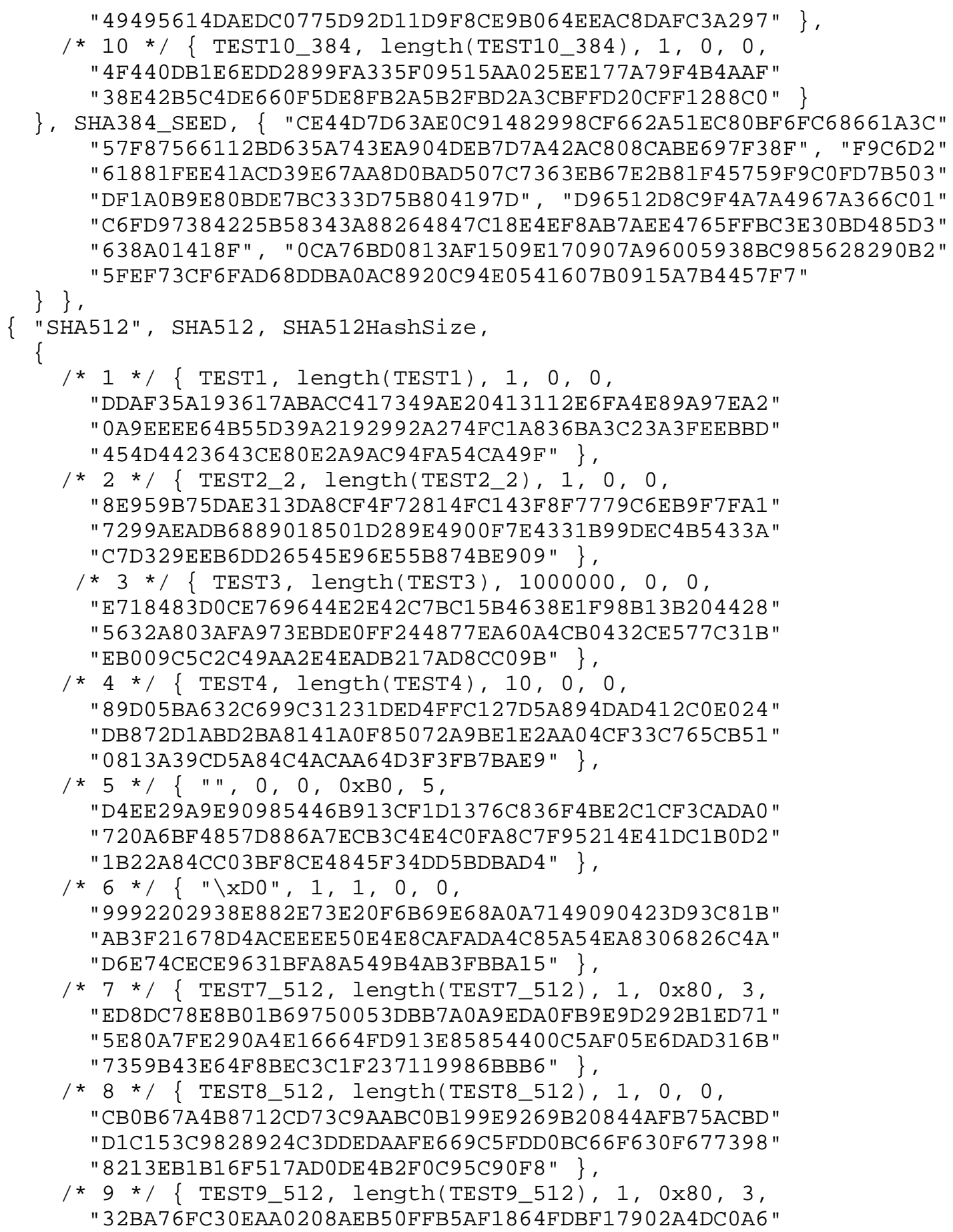




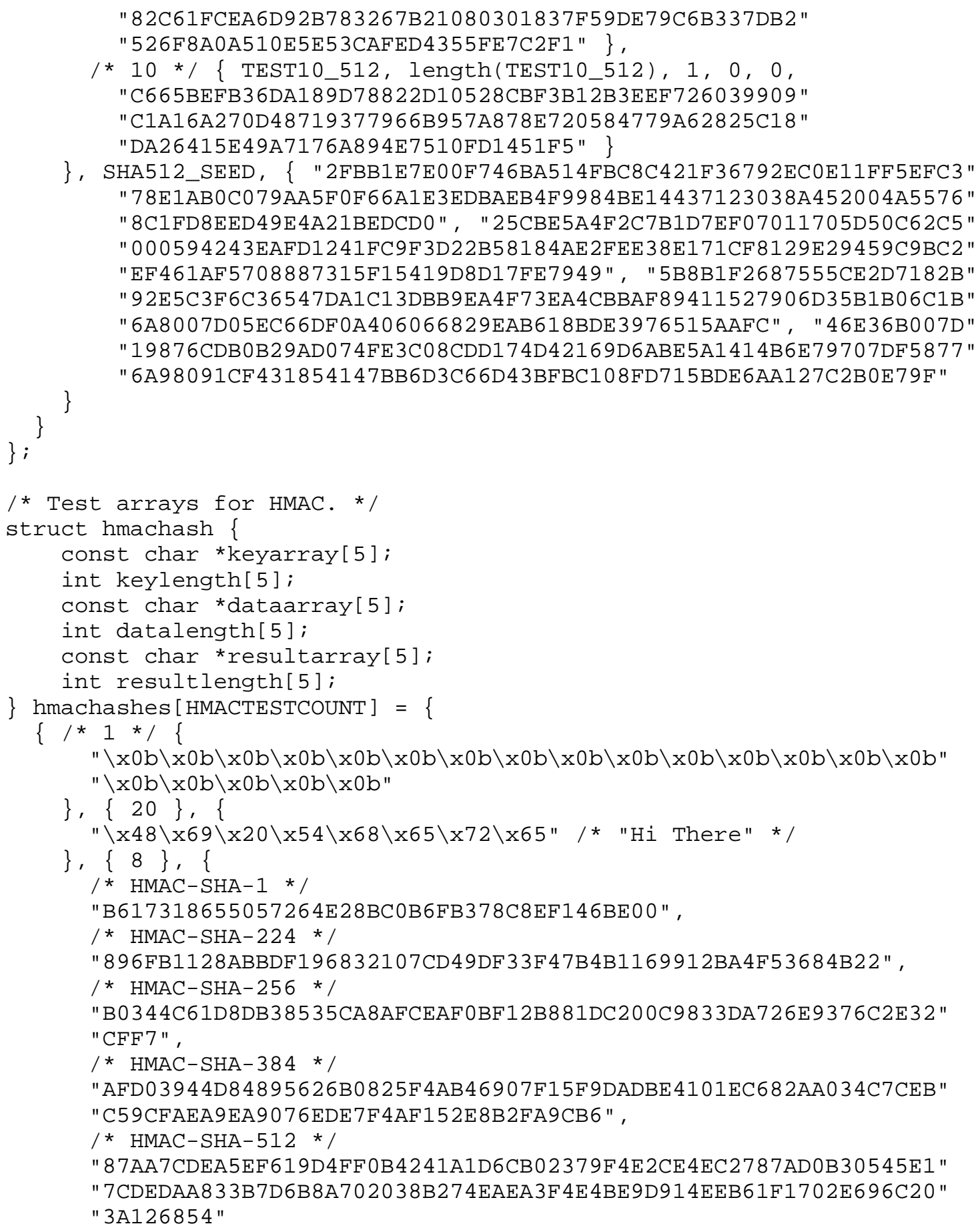




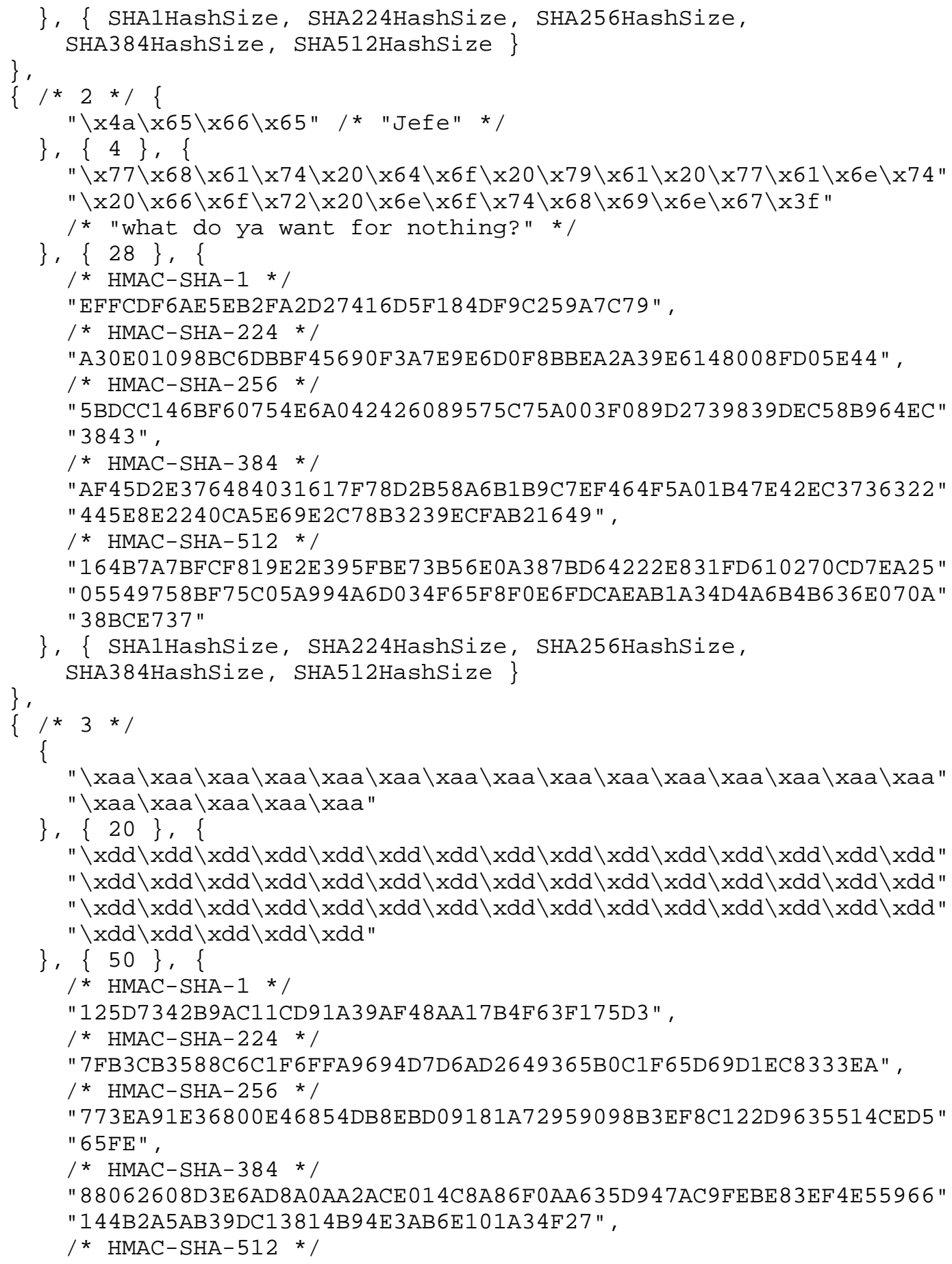




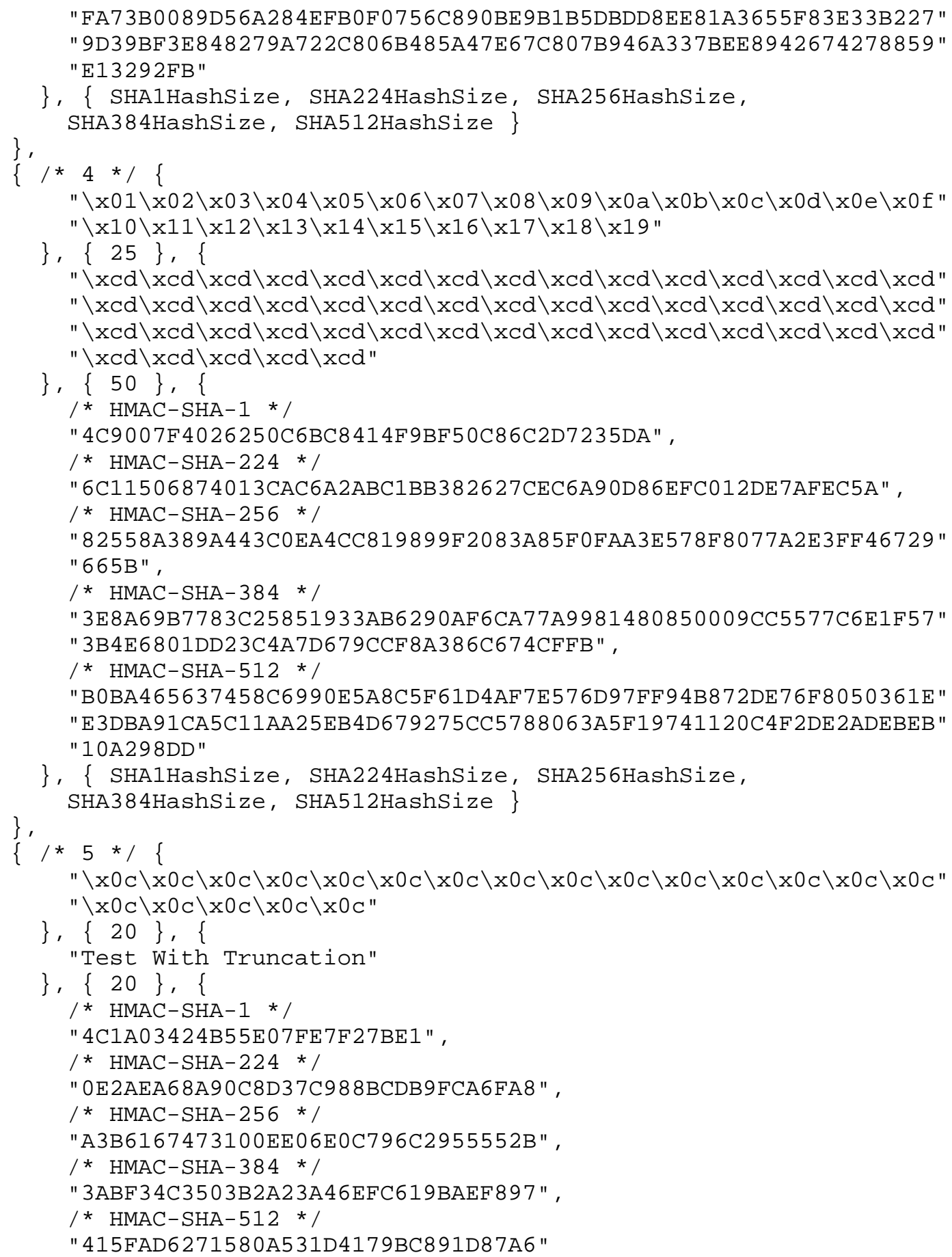




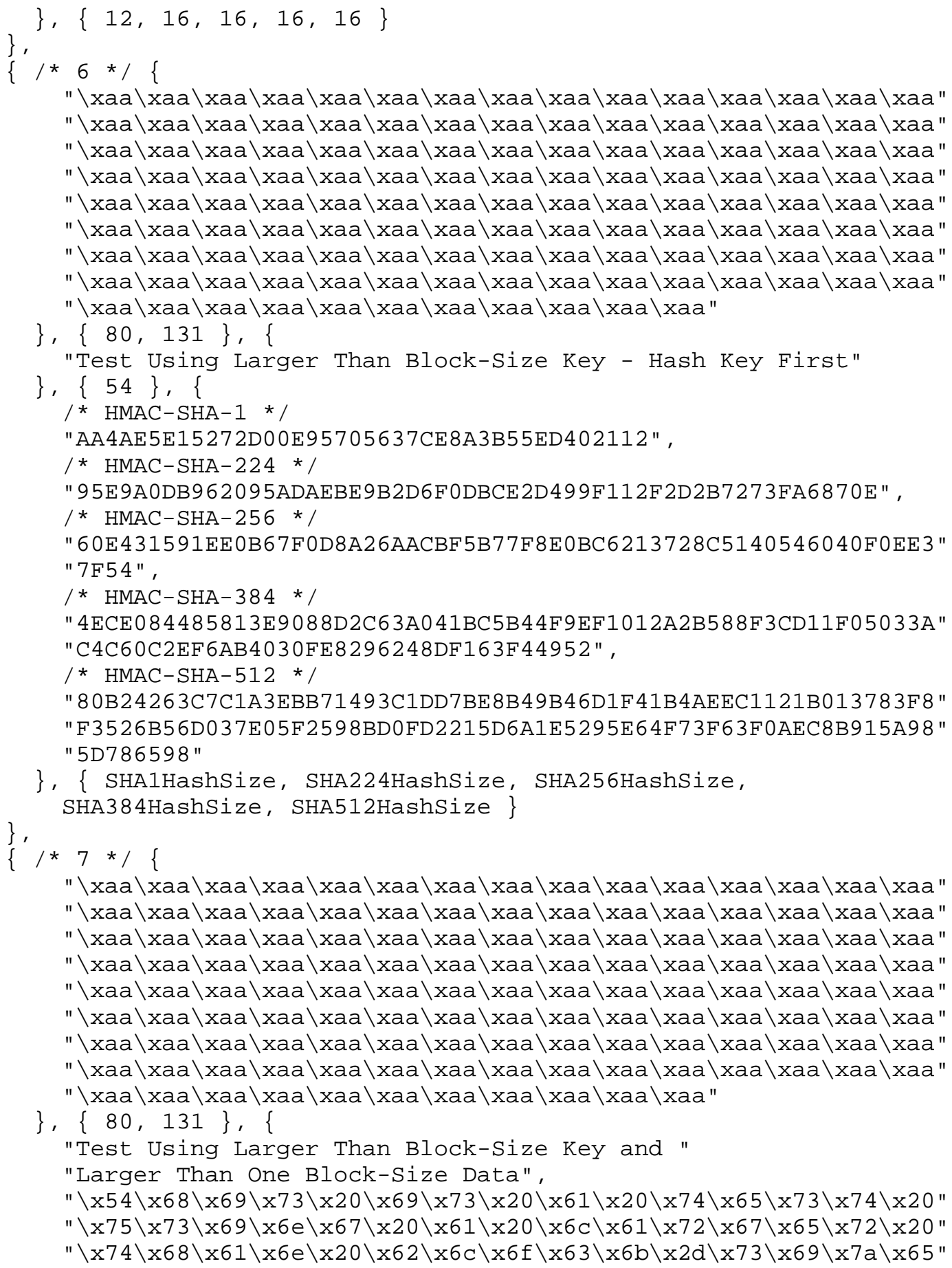




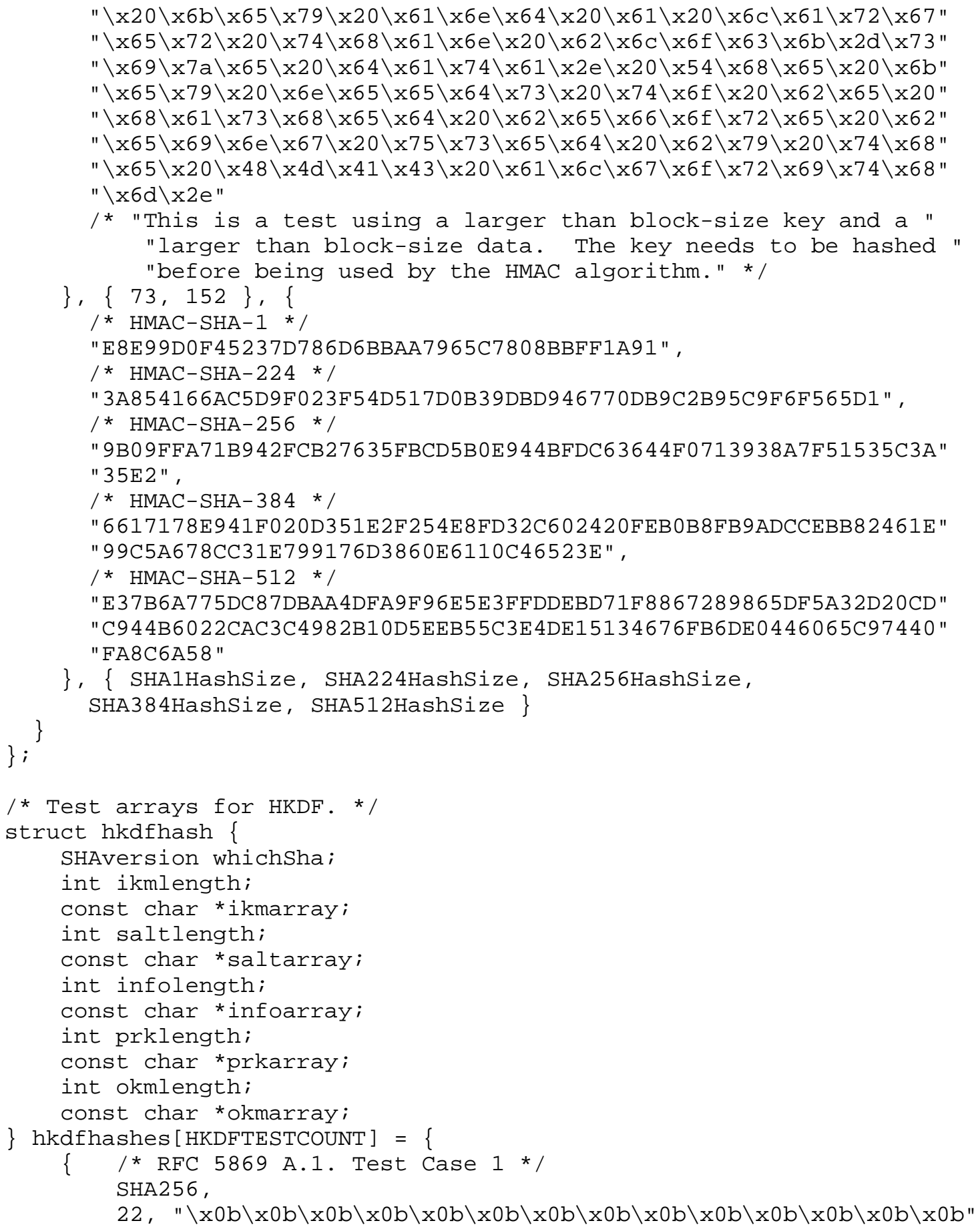




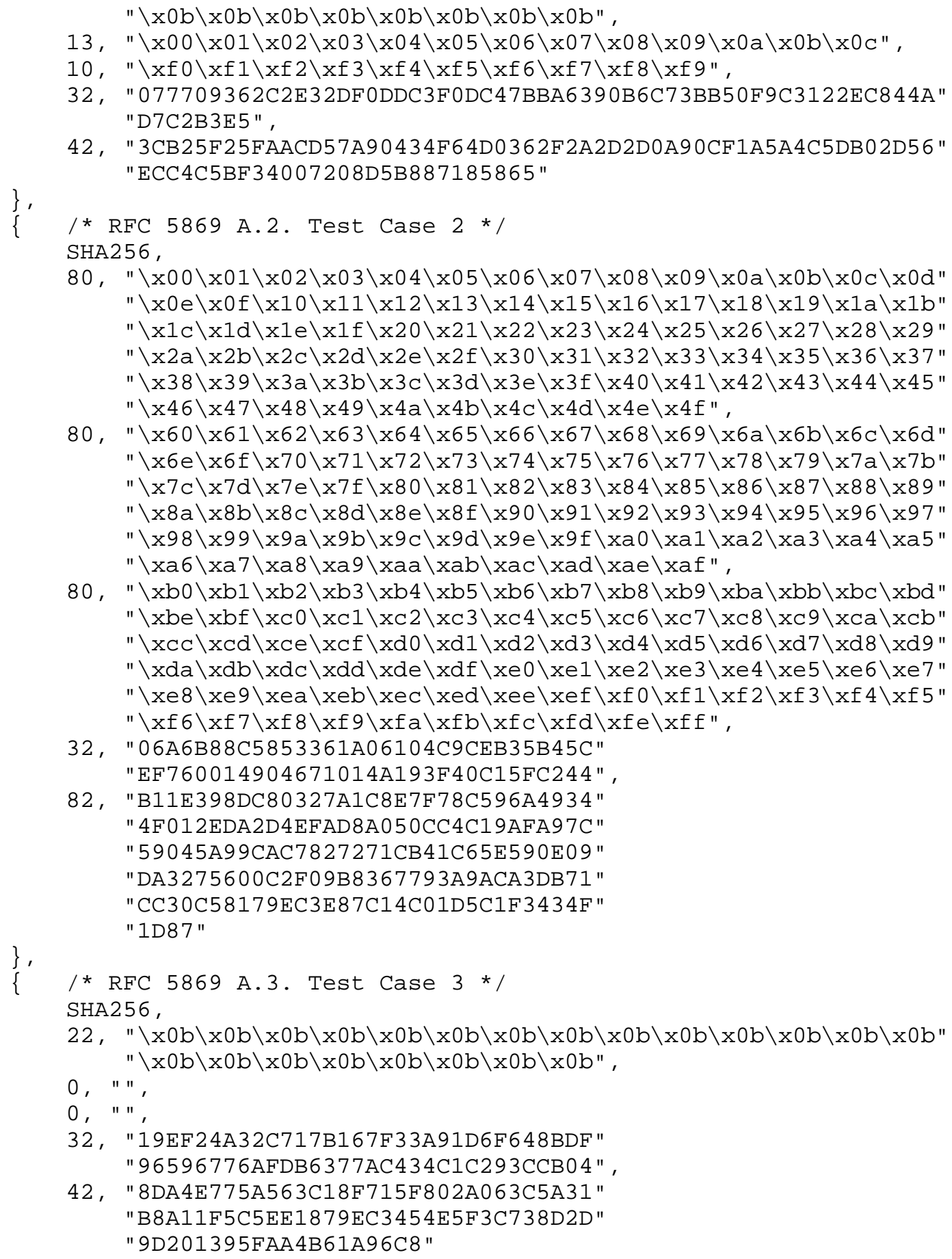




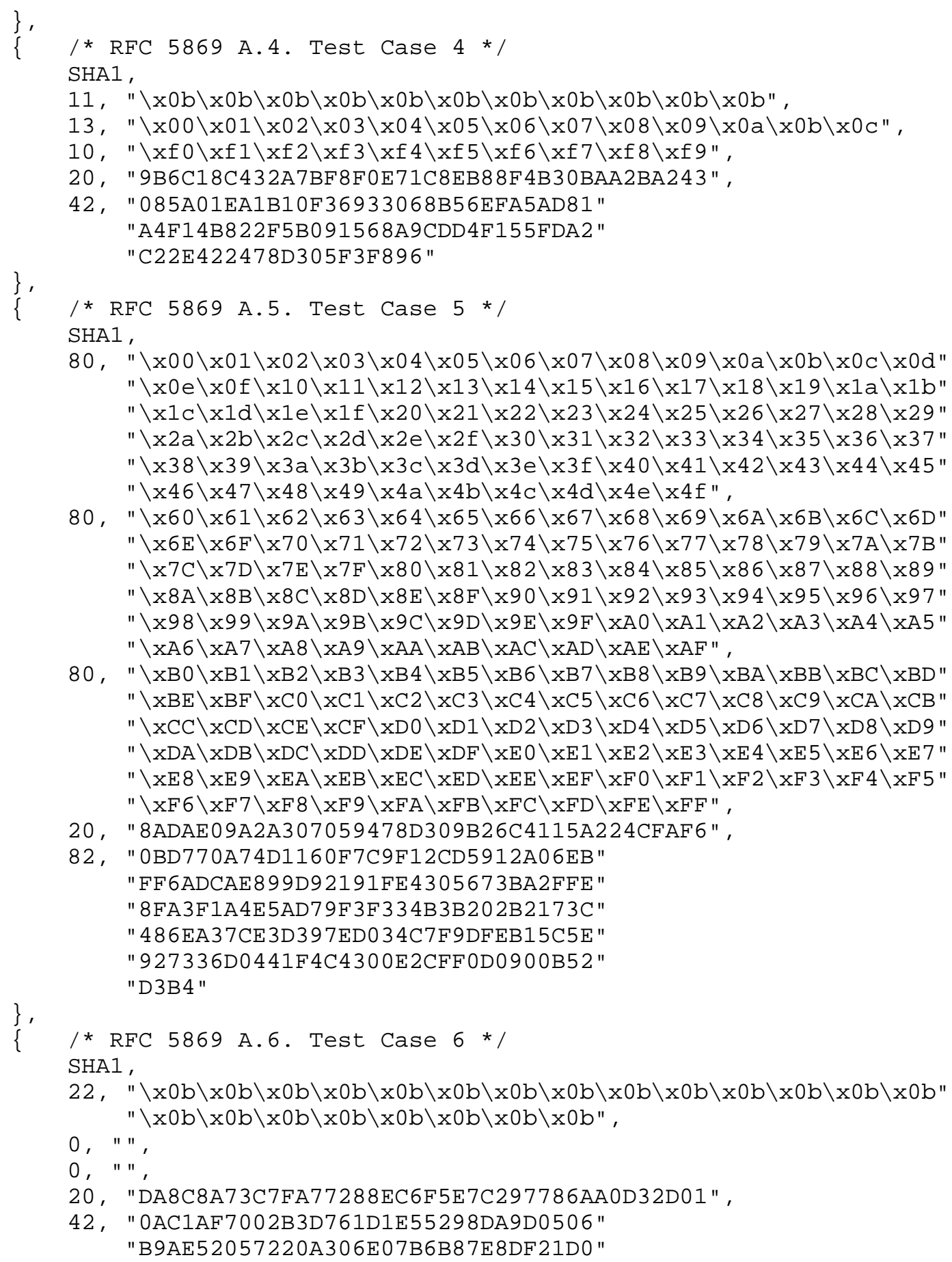




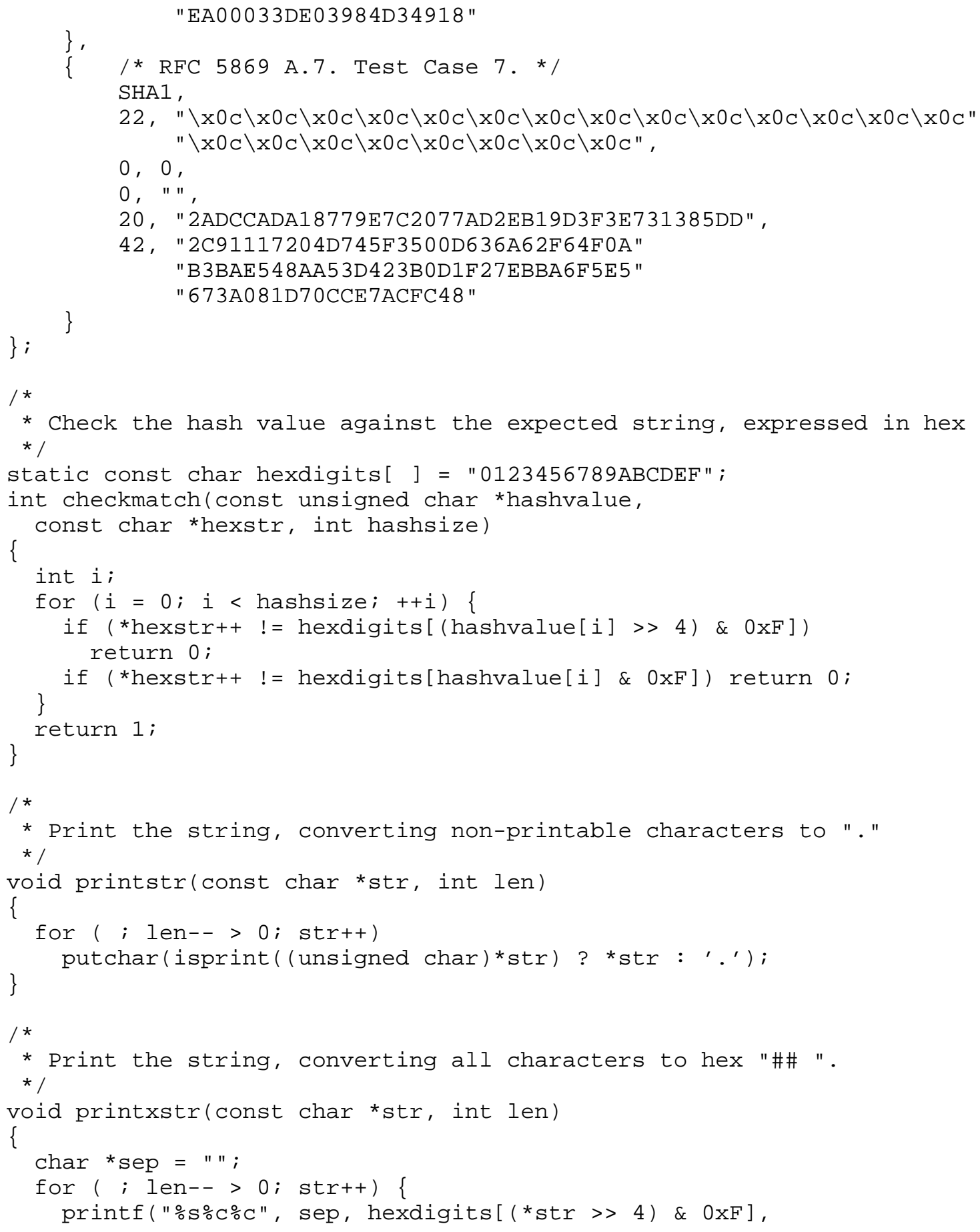




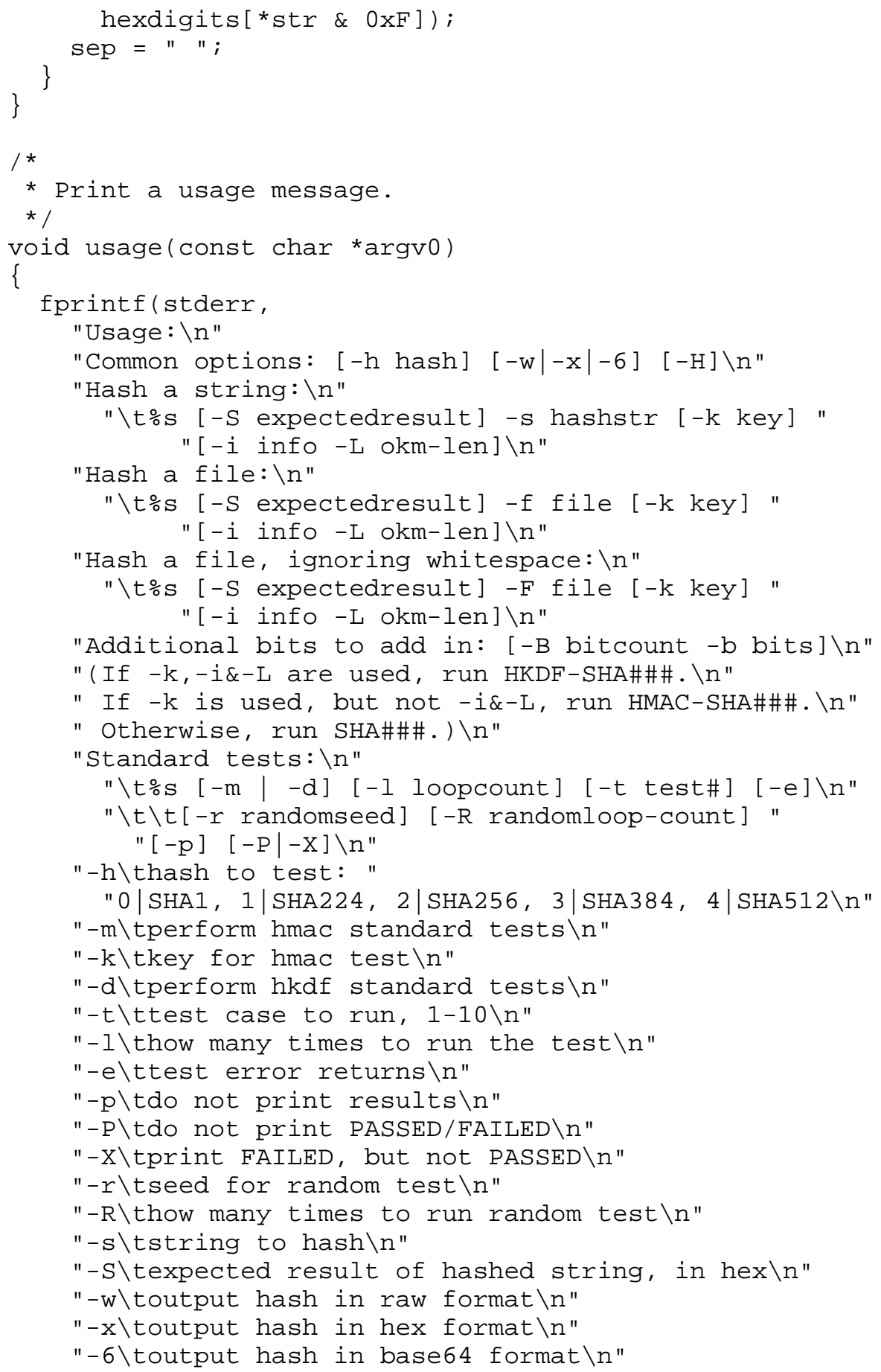




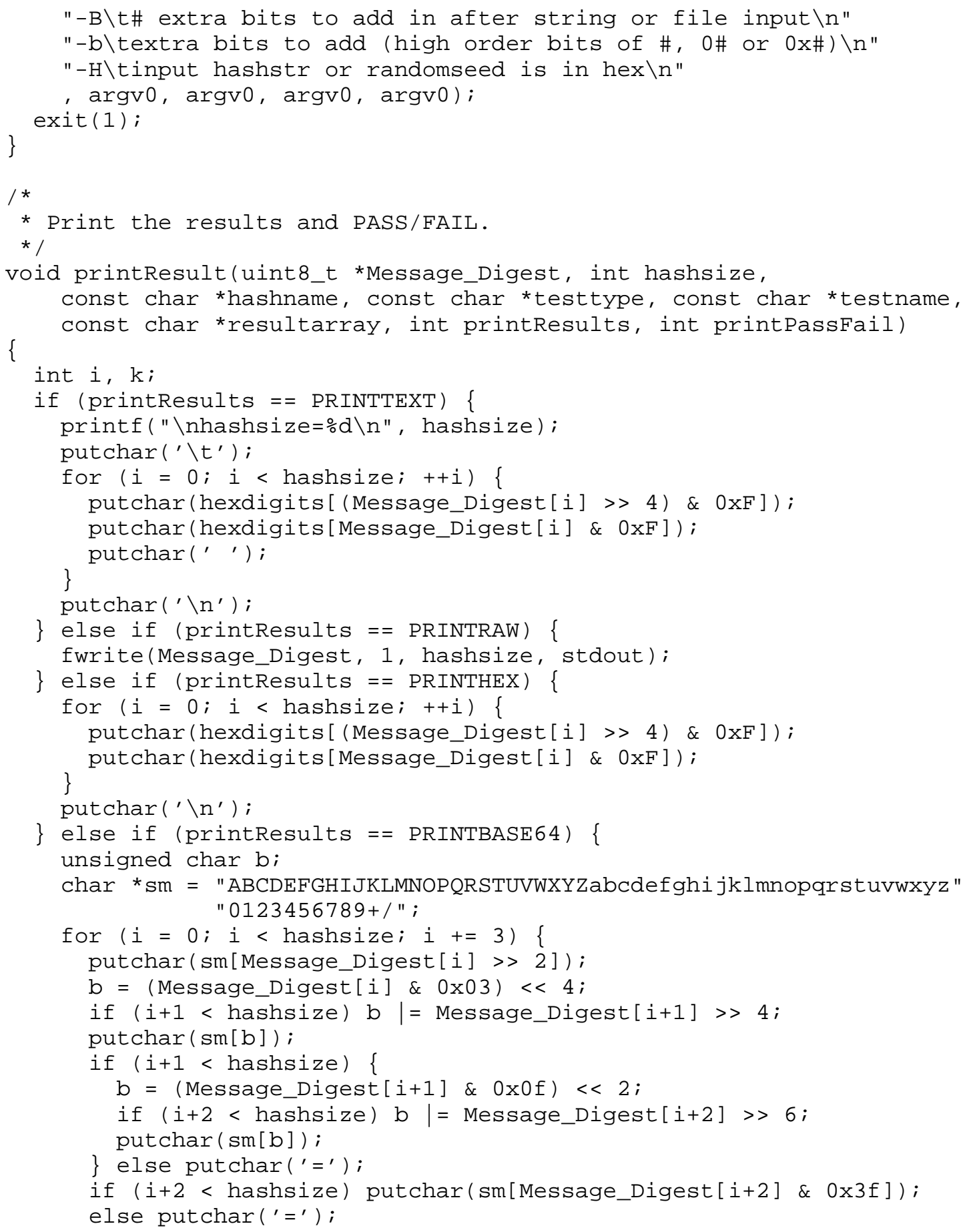




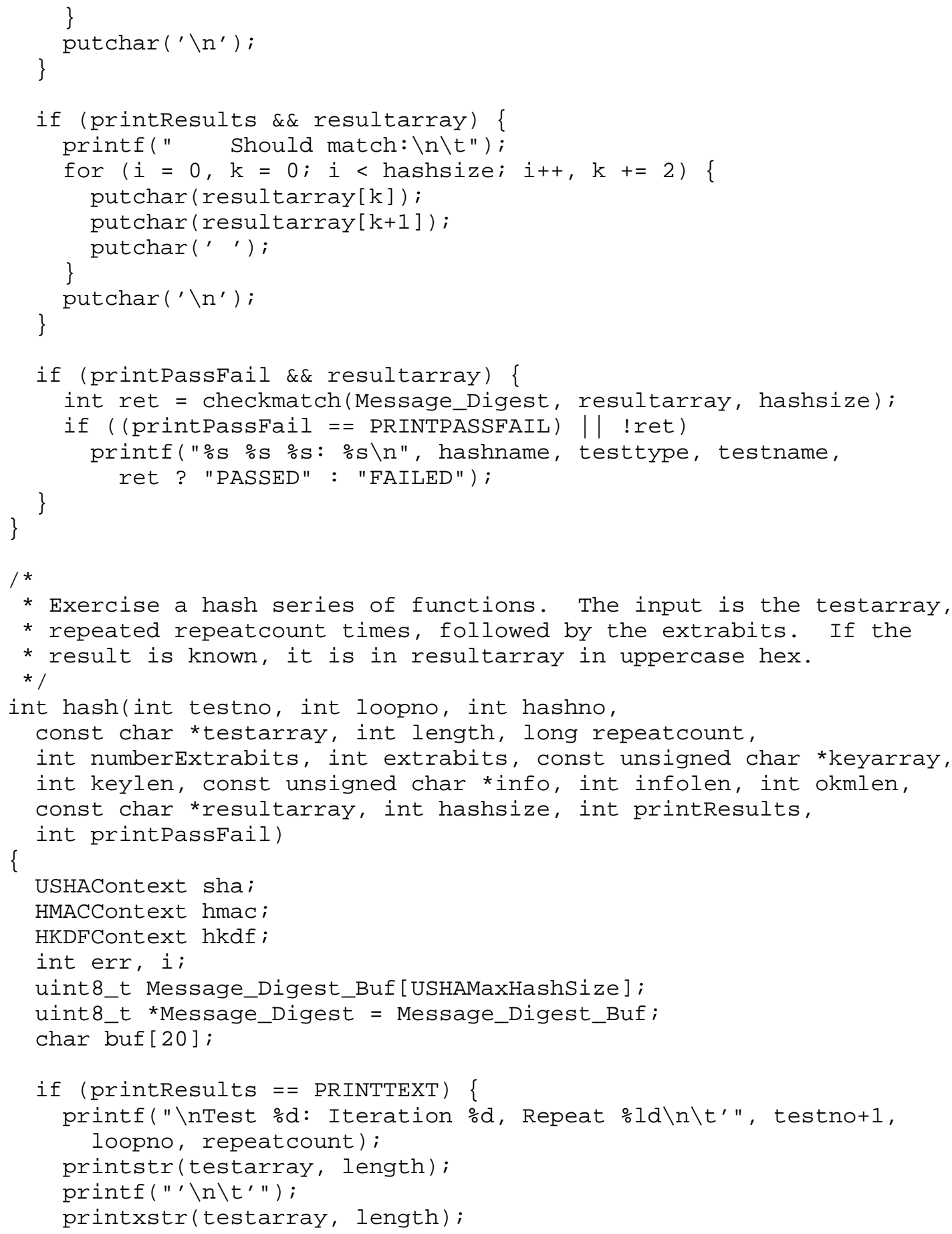




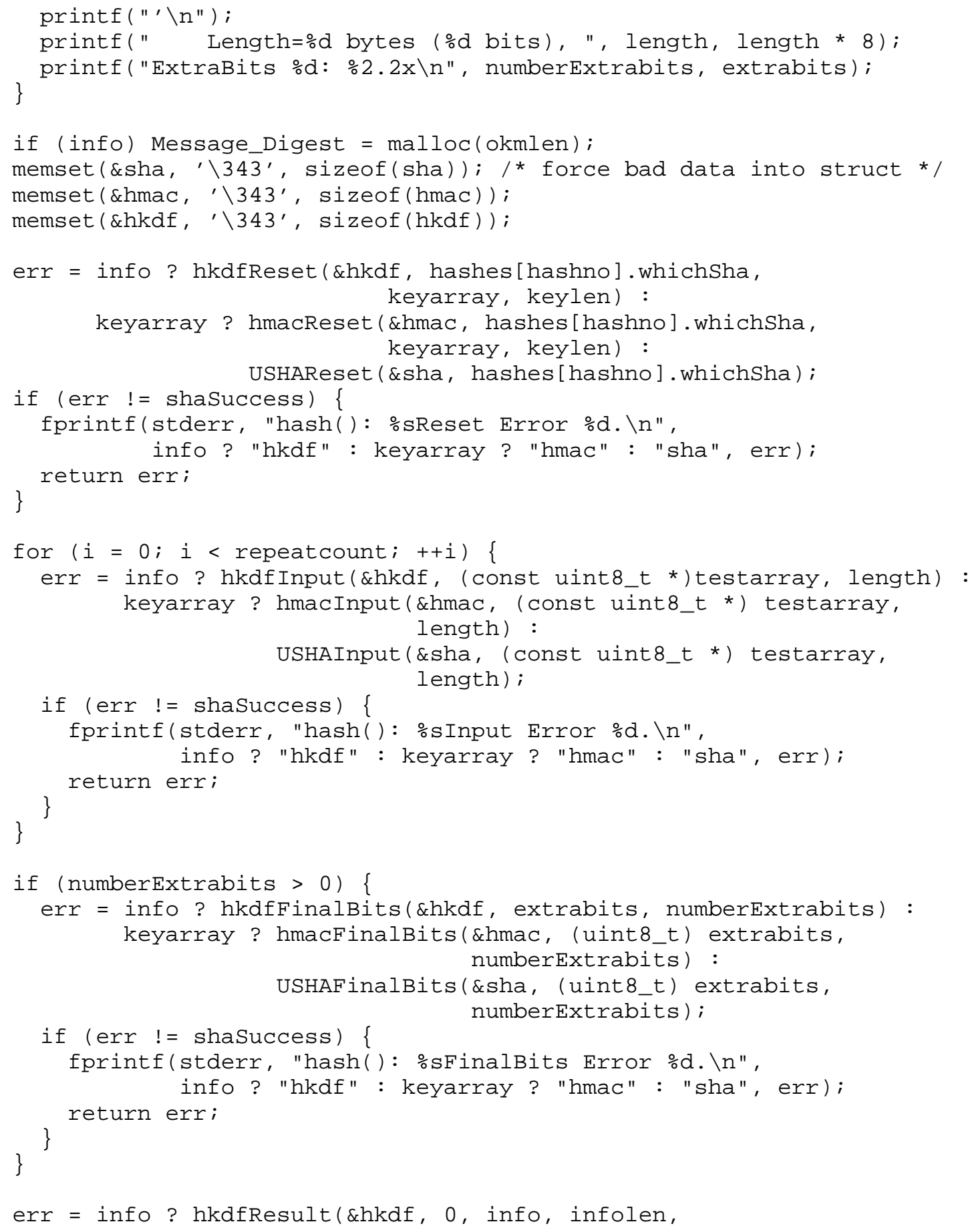




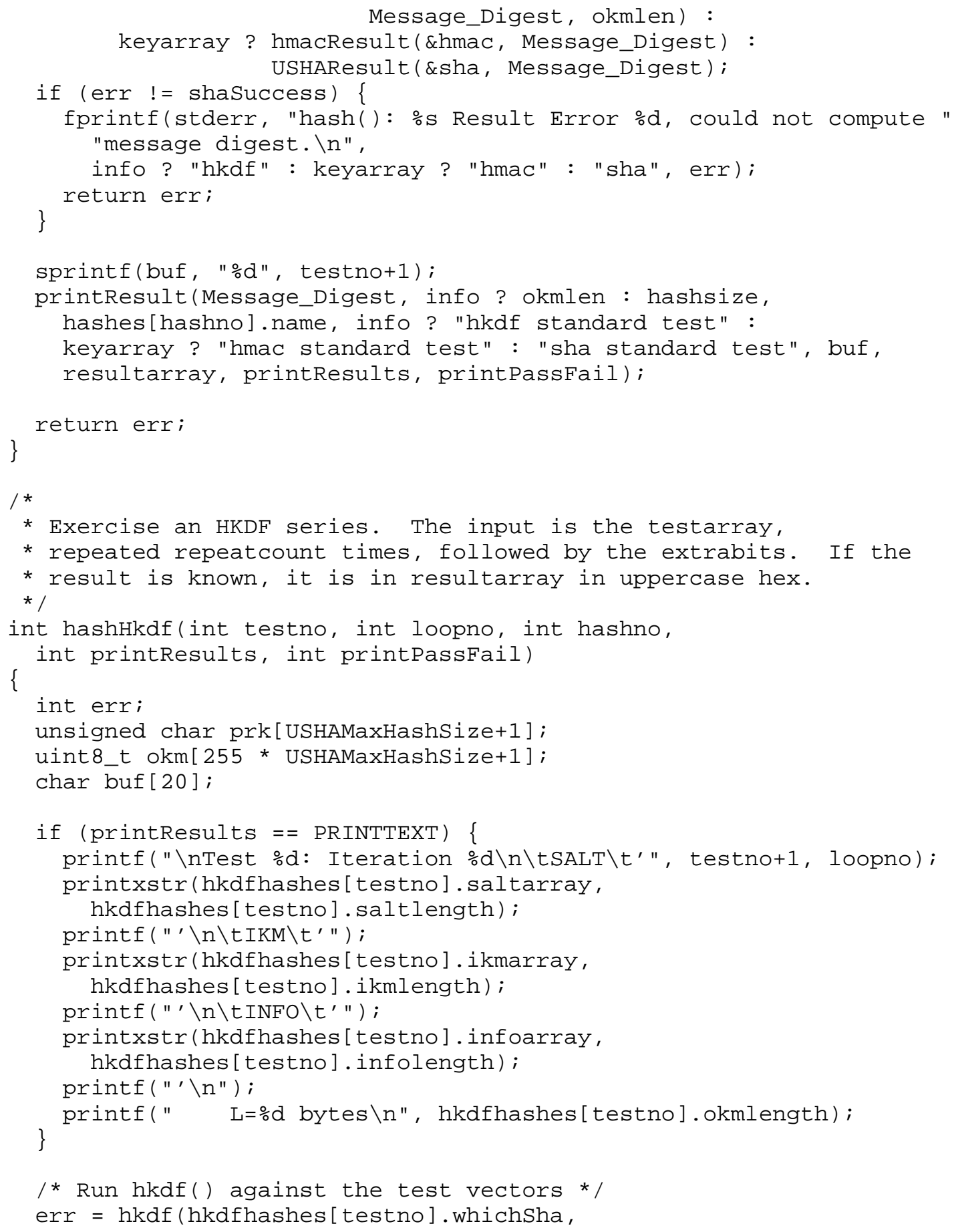




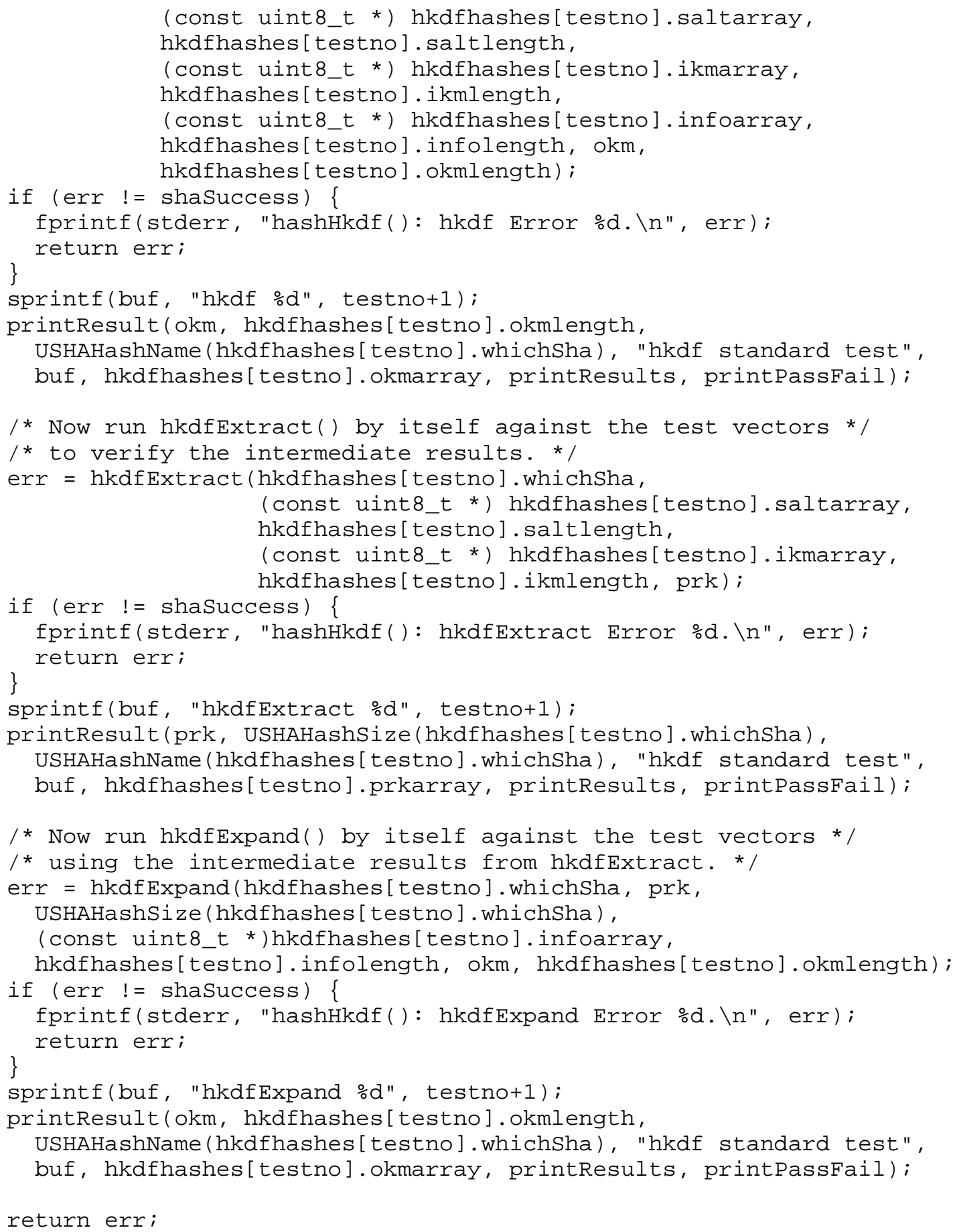




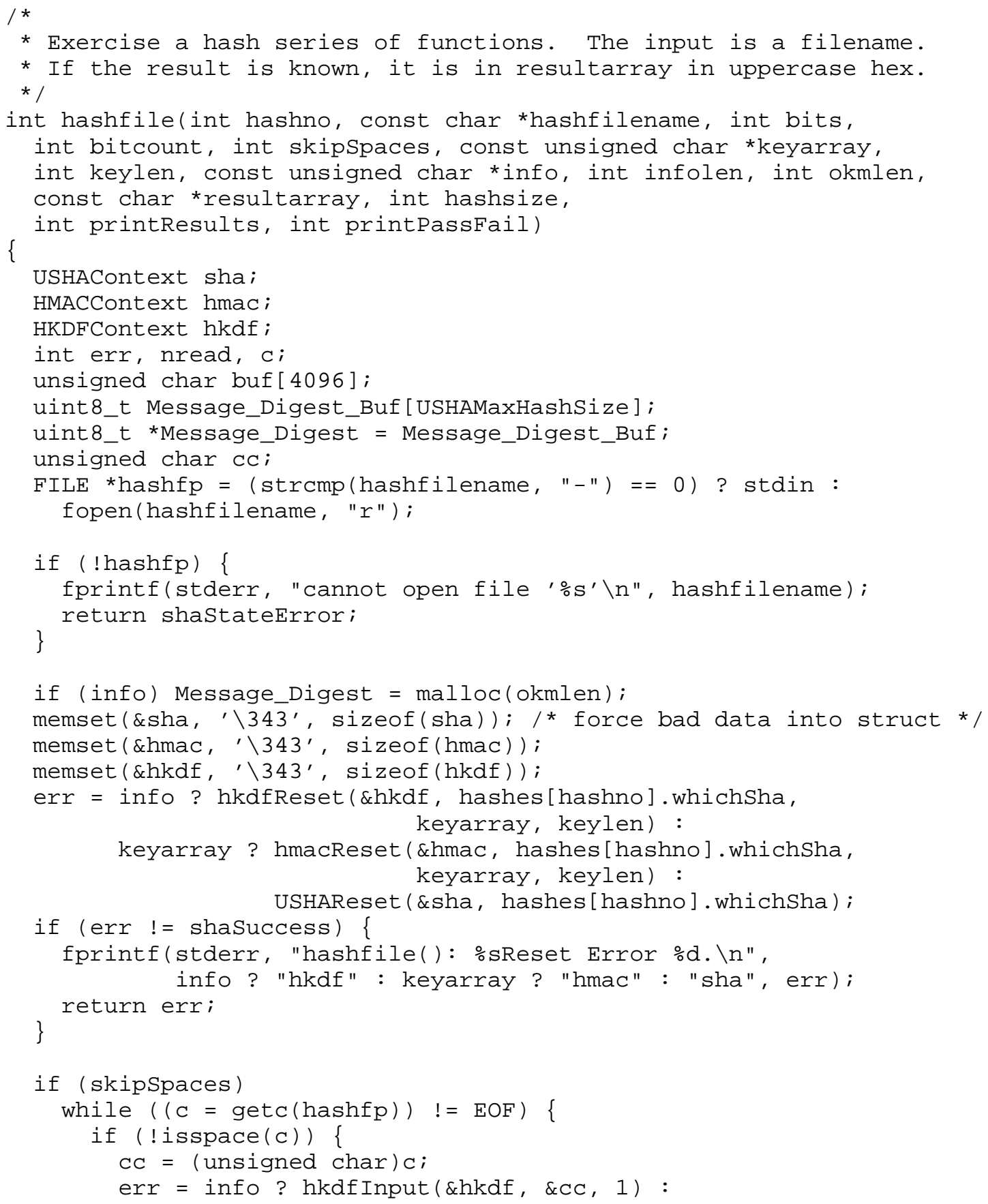




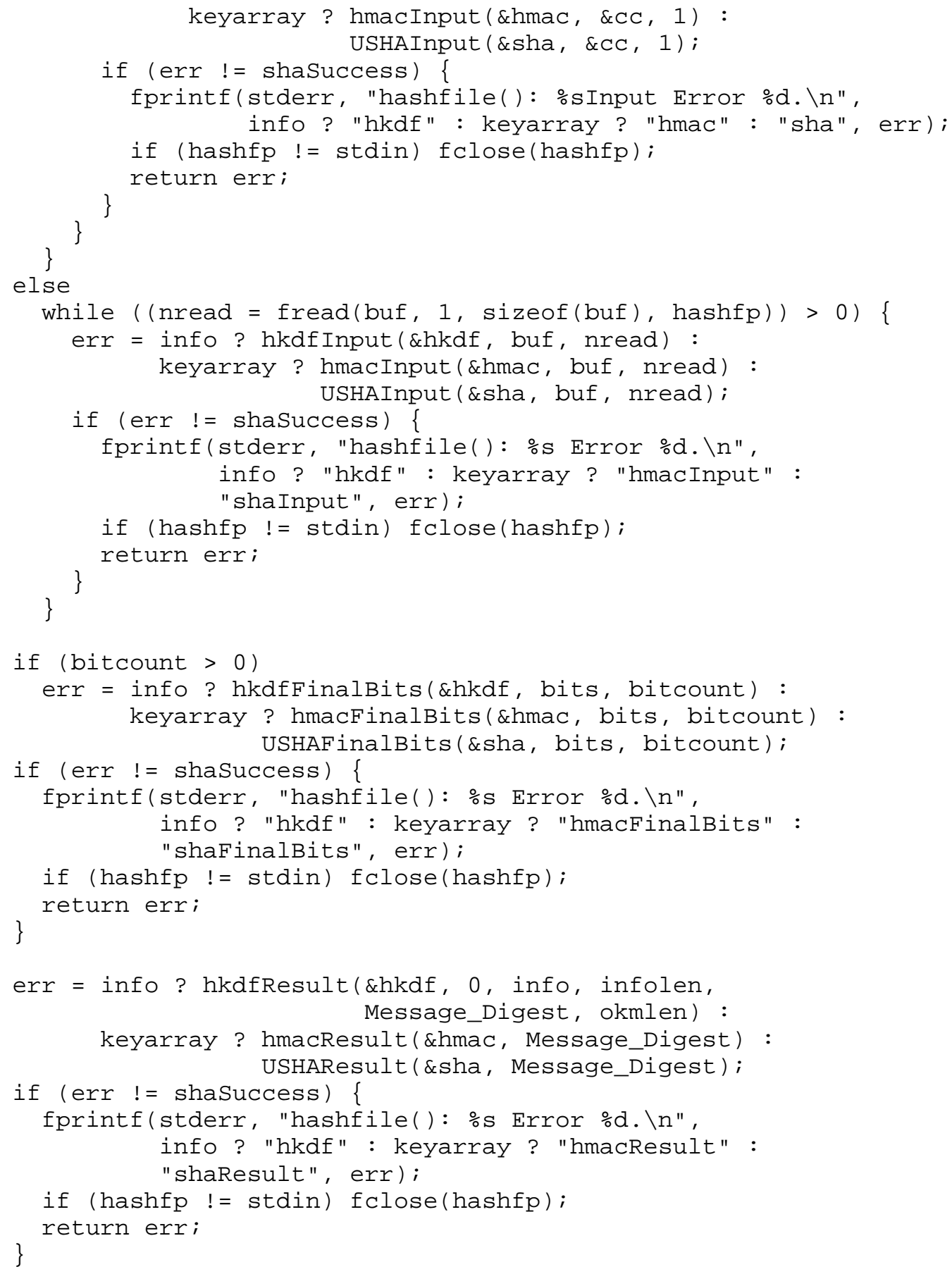




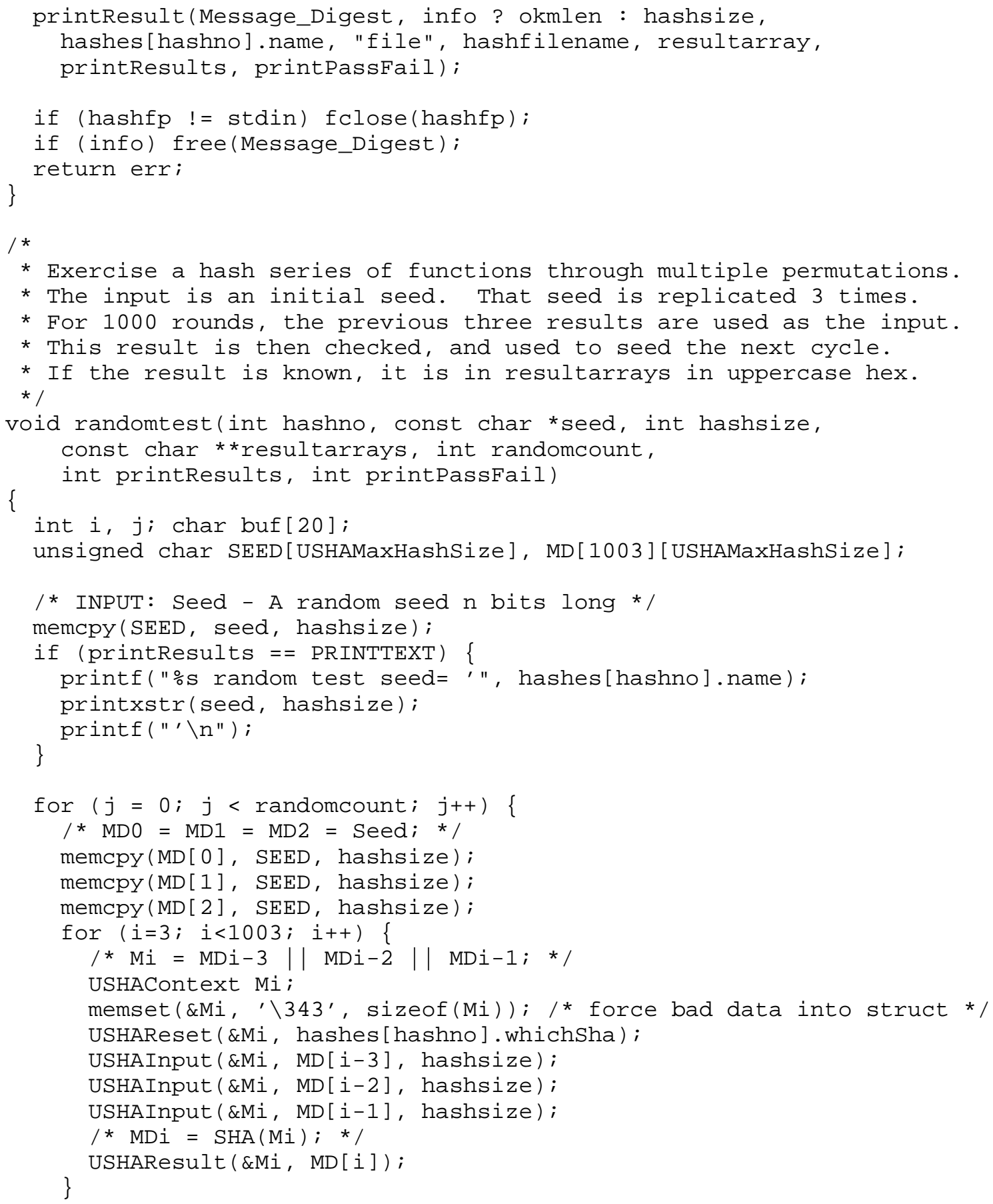




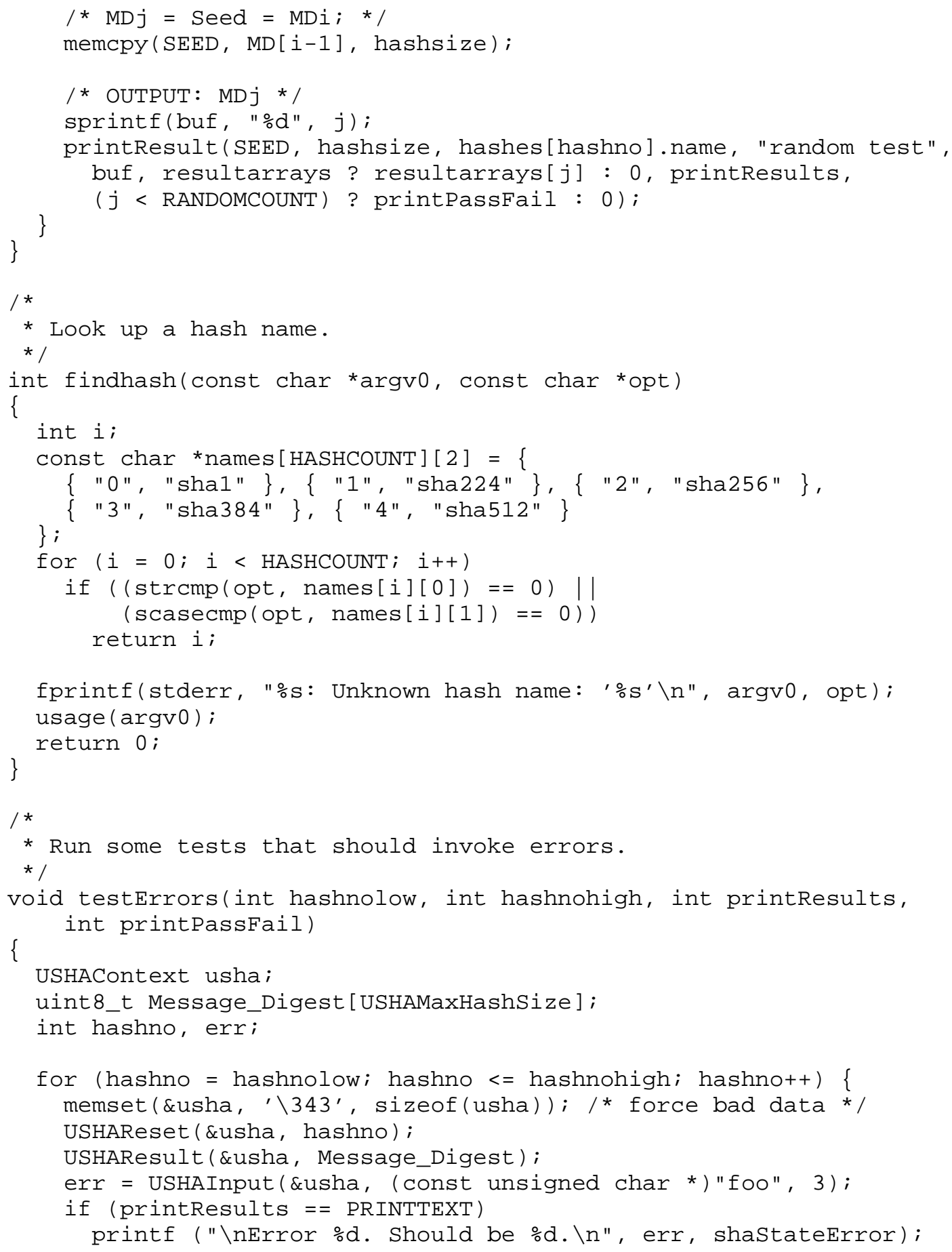




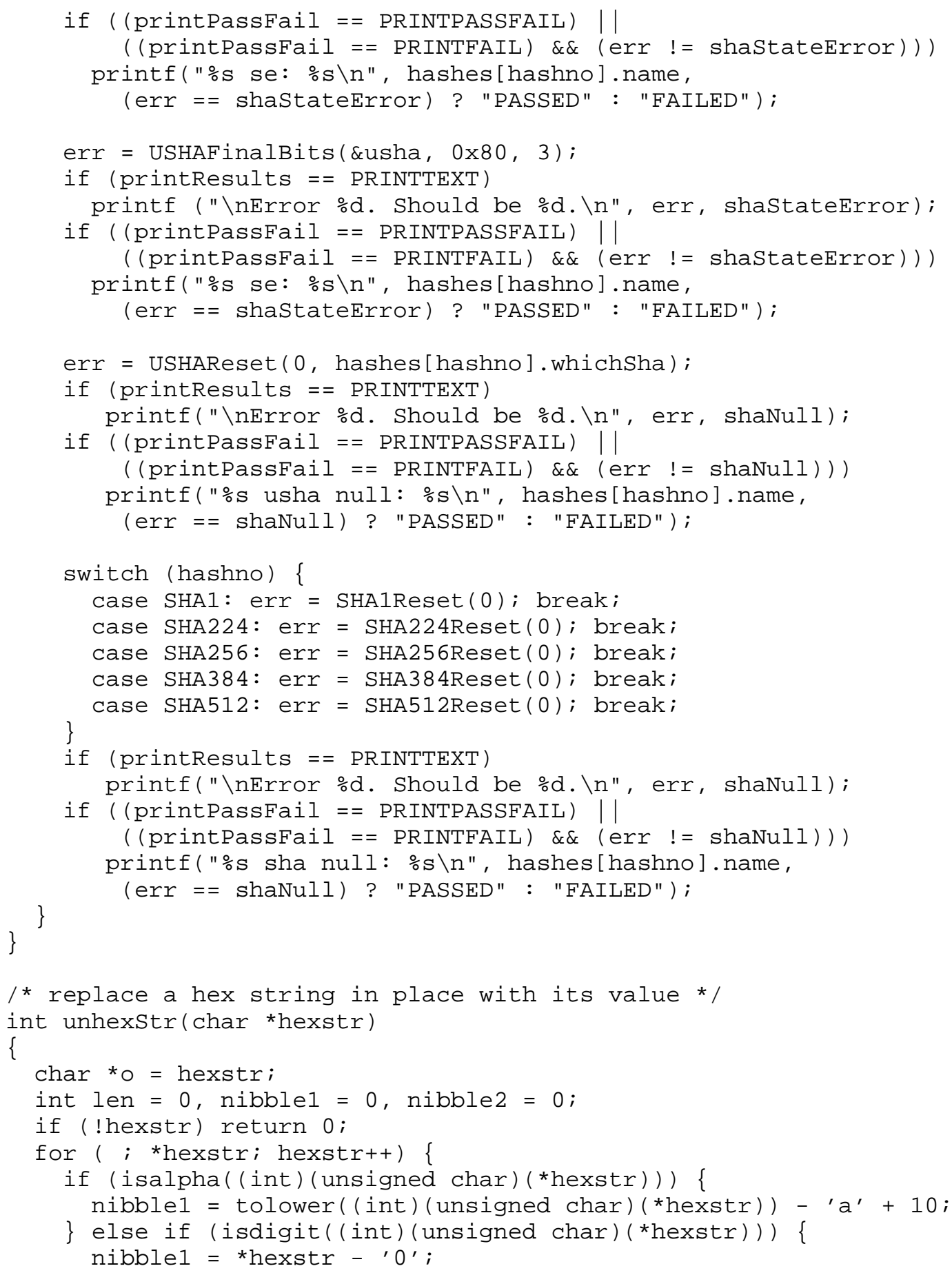




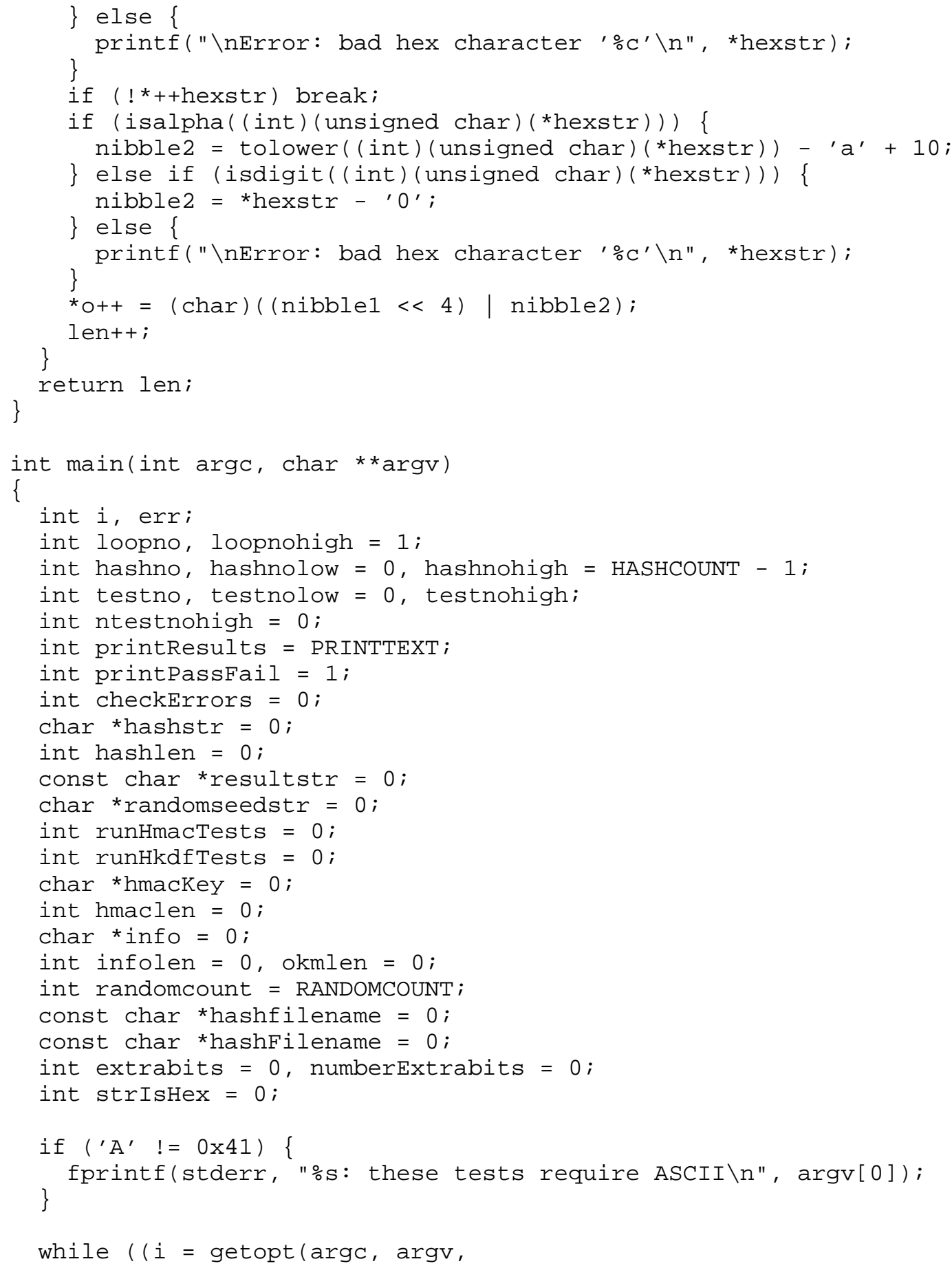




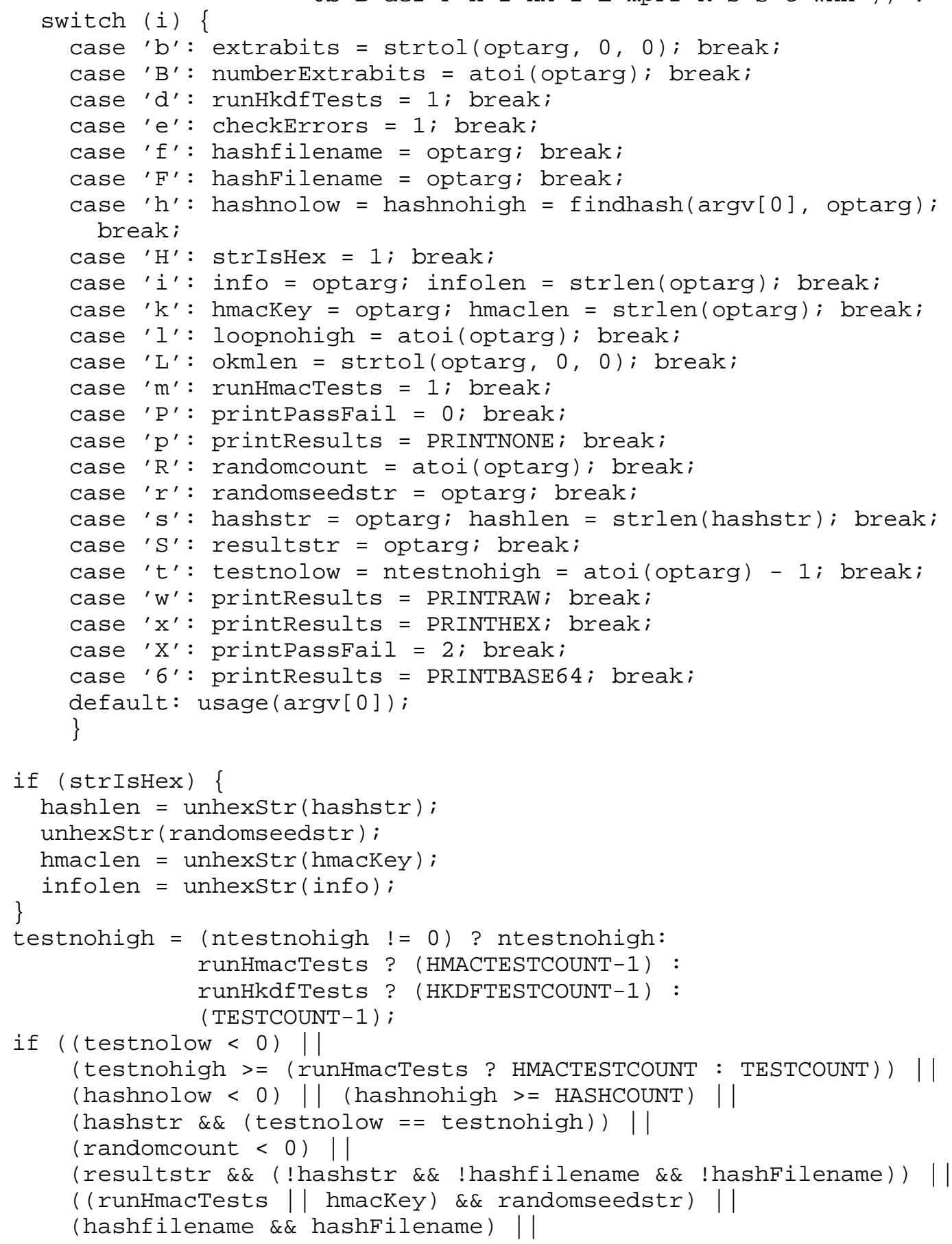




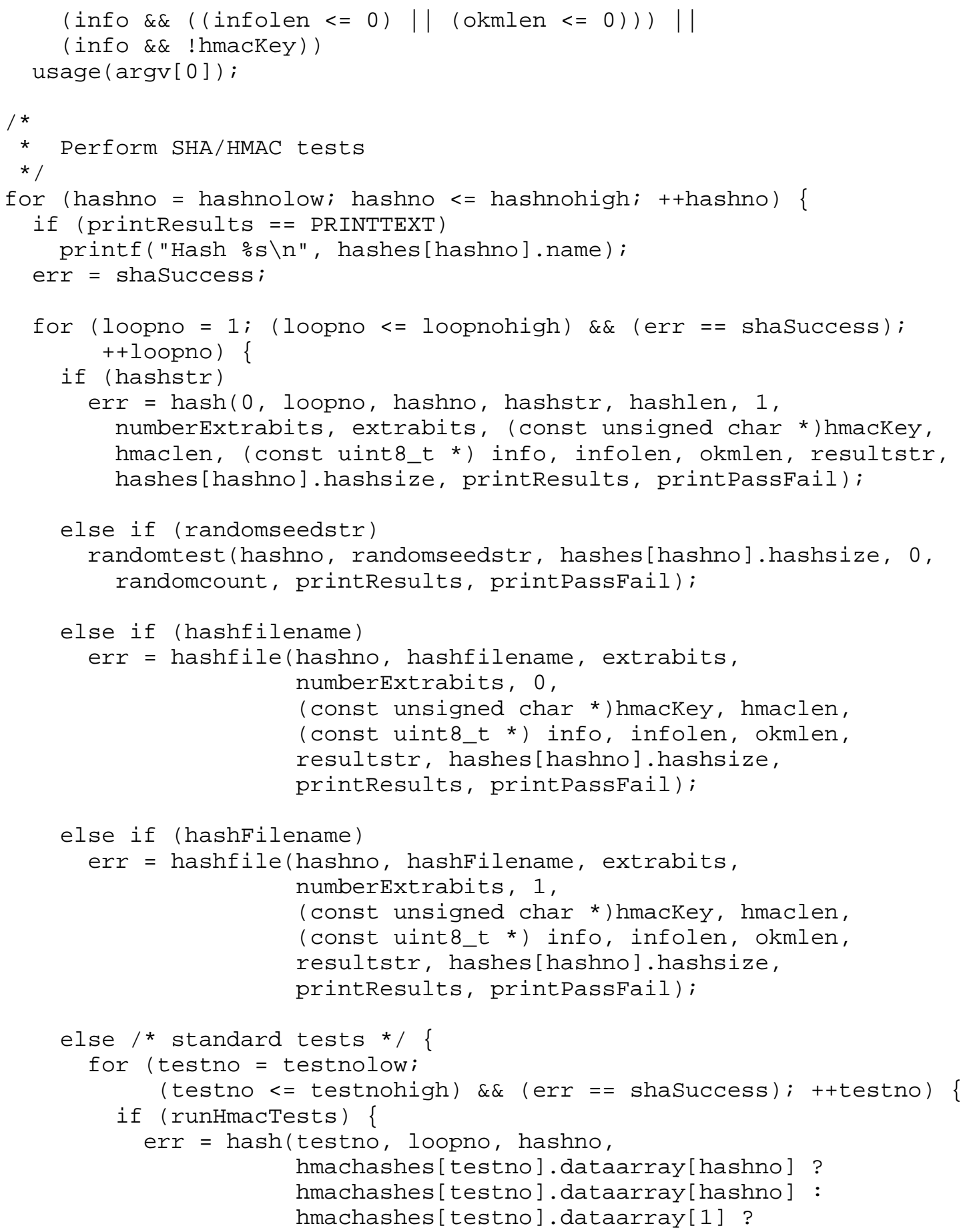




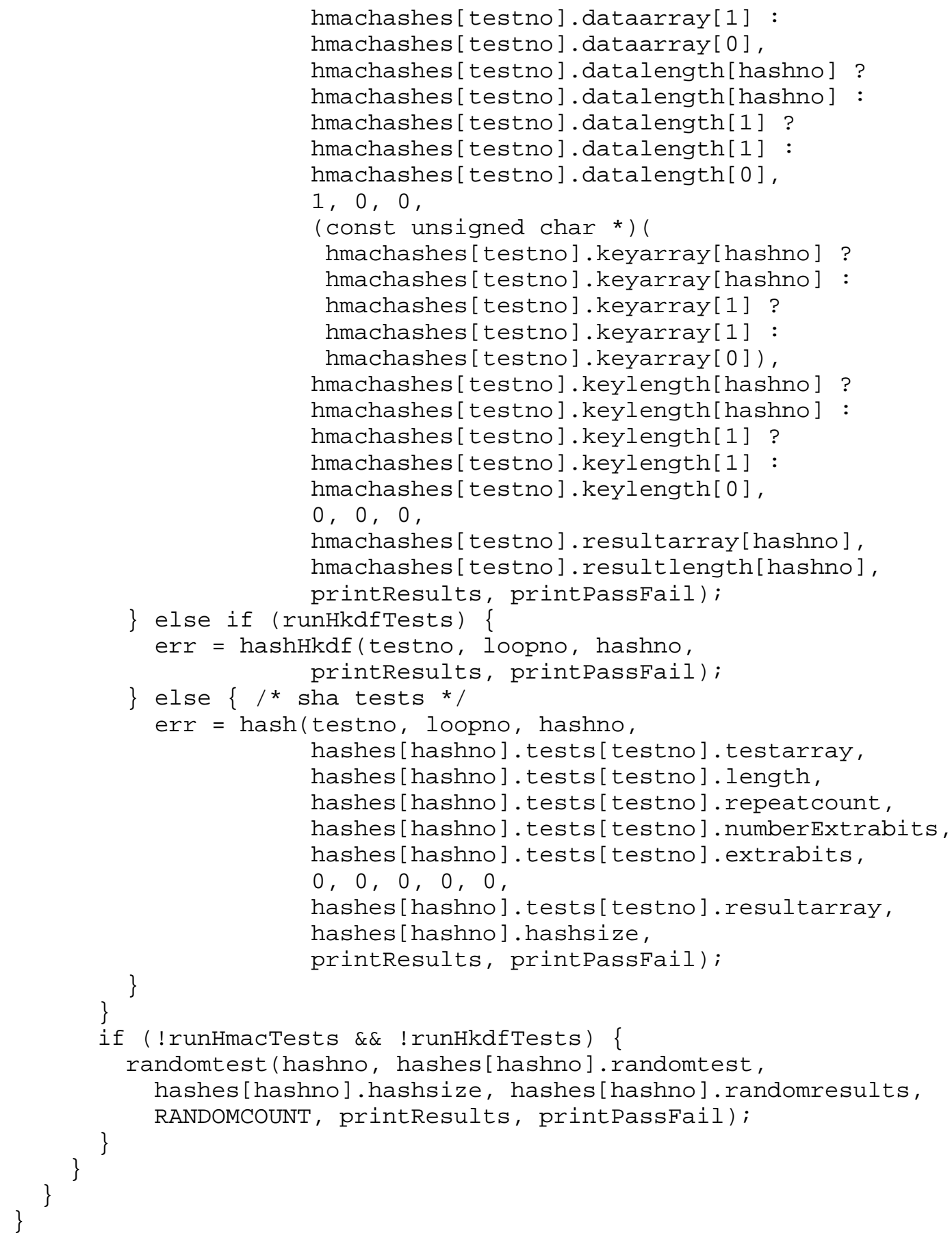




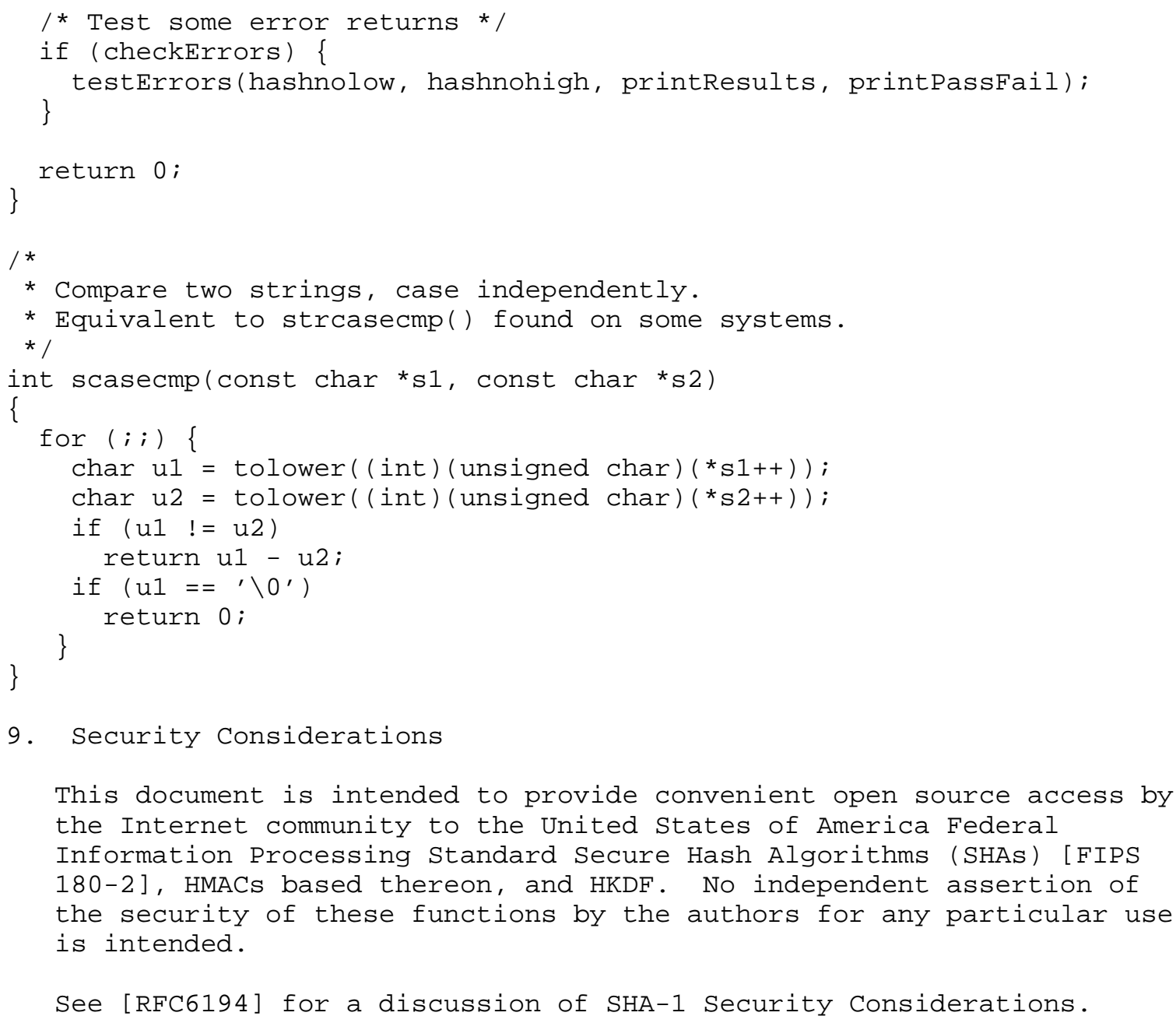

9. Security Considerations

This document is intended to provide convenient open source access by the Internet community to the United States of America Federal Information Processing Standard Secure Hash Algorithms (SHAs) [FIPS 180-2], HMACs based thereon, and HKDF. No independent assertion of the security of these functions by the authors for any particular use is intended.

See [RFC6194] for a discussion of SHA-1 Security Considerations.

10. Acknowledgements

Thanks for the corrections to [RFC4634] that were provided by Alfred Hoenes and Jan Andres and to Alfred's comments on the document hereof.

Also to the following in alphabetic order, whose comments lead to improvements in the document: James Carlson, Russ Housley, Tero Kivinen, Juergen Quittek, and Sean Turner. 
11. References

11.1. Normative References

[RFC2104] Krawczyk, H., Bellare, M., and R. Canetti, "HMAC: KeyedHashing for Message Authentication", RFC 2104, February 1997.

[RFC5869] Krawczyk, H. and P. Eronen, "HMAC-based Extract-and-Expand Key Derivation Function (HKDF)", RFC 5869, May 2010.

[SHS] "Secure Hash Standard", United States of American, National Institute of Science and Technology, Federal Information Processing Standard (FIPS) 180-3, http://csrc.nist.gov/publications/fips/fips180-3/ fips180-3_final.pdf.

[US-ASCII] ANSI, "USA Standard Code for Information Interchange", X3.4, American National Standards Institute: New York, 1968 .

\subsection{Informative References}

[RFC3174] Eastlake 3rd, D. and P. Jones, "US Secure Hash Algorithm 1 (SHA1)", RFC 3174, September 2001.

[RFC3874] Housley, R., "A 224-bit One-way Hash Function: SHA-224", RFC 3874, September 2004.

[RFC4055] Schaad, J., Kaliski, B., and R. Housley, "Additional Algorithms and Identifiers for RSA Cryptography for use in the Internet X.509 Public Key Infrastructure Certificate and Certificate Revocation List (CRL) Profile", RFC 4055, June 2005.

[RFC4086] Eastlake 3rd, D., Schiller, J., and S. Crocker, "Randomness Requirements for Security", BCP 106, RFC 4086, June 2005.

[RFC4634] Eastlake 3rd, D. and T. Hansen, "US Secure Hash Algorithms (SHA and HMAC-SHA)", RFC 4634, July 2006.

[RFC6194] Polk, T., Chen, L., Turner, S., and P. Hoffman, "Security Considerations for the SHA-0 and SHA-1 Message-Digest Algorithms", RFC 6194, March 2011. 
[SHAVS] "The Secure Hash Algorithm Validation System (SHAVS)", http://csrc.nist.gov/groups/STM/cavp/documents/shs/ SHAVS.pdf, July 2004 . 
Appendix: Changes from RFC 4634

The following changes were made to RFC 4634 to produce this document:

1. Add code for HKDF and brief text about HKDF with pointer to [RFC5869].

2. Fix numerous errata filed against [RFC4634] as included below. Note that in no case did the old code return an incorrect hash value.

2.a. Correct some of the error return values which has erroneously been "shaNull" to the correct "shalnputTooLong" error.

2.b. Update comments and variable names within the code for consistency and clarity and other editorial changes.

2.C. The previous code for SHA-384 and SHA-512 would stop after $2^{\wedge} 93$ bytes (2^96 bits). The fixed code handles up to $2^{\wedge} 125$ bytes $\left(2^{\wedge} 128\right.$ bits $)$.

2.d. Add additional error checking including a run time check in the test driver to detect attempts to run the test driver after compilation using some other character set instead of [US-ASCII].

3. Update boilerplate, remove special license in [RFC4634] as new boilerplate mandates simplified BSD license.

4. Replace MIT version of getopt with new code to satisfy IETF incoming and outgoing license restrictions.

5. Add references to [RFC6194].

6. Other assorted editorial improvements. 


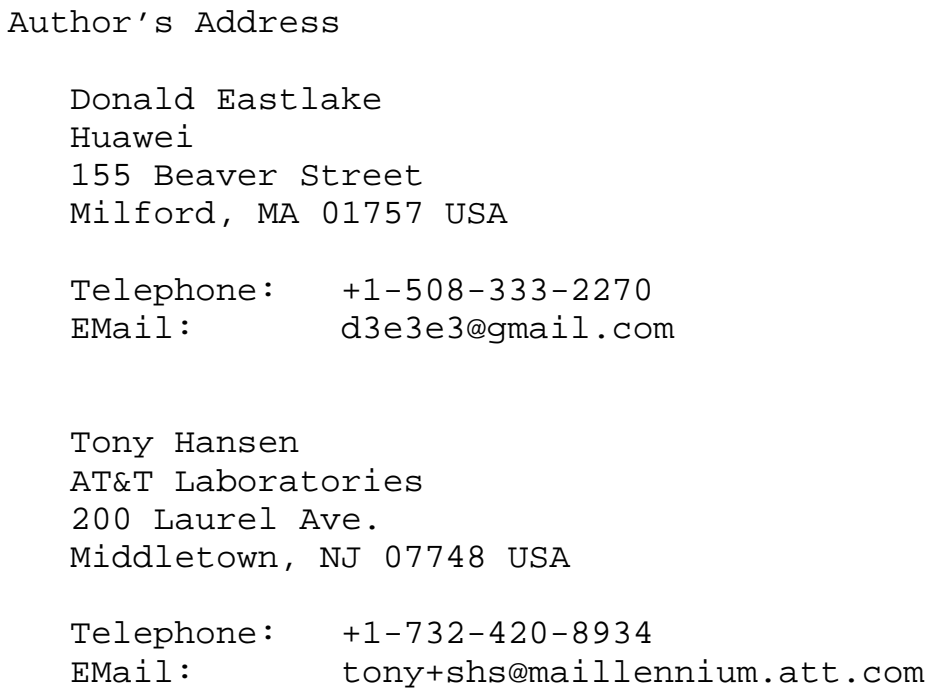

